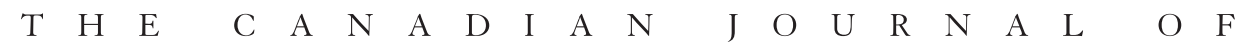

INFECTIOUS DISEASES \& MEDICAL MICROBIOLOGY

JOUR A L C A N A I EN DES MALADIES INFECTIEUSES ET DE LA MICROBIOLOGIE MEDICALE

Spring $2011 \cdot$ VoIume 22 - Supplement A

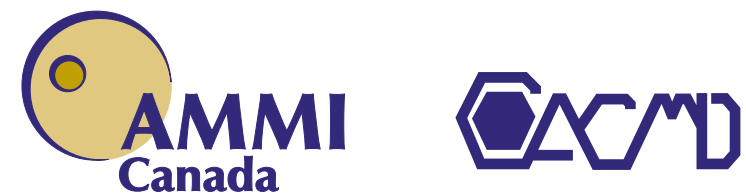

\title{
AMMI Canada - CACMID Annual Conference
}

April 7-9, 2011, Montreal, Quebec

\section{ORAL PRESENTATIONS}

Thursday, April 7, 2011

\begin{tabular}{|rr}
\hline Session A & A1-A6 \\
\hline Session B & B1-B7 \\
\hline Session C & C1-C6 \\
\hline Session D & D1-D6
\end{tabular}

Friday, April 8, 2011

\begin{tabular}{|rr}
\hline Session E & E1-E6 \\
\hline Session F & F1-F6 \\
\hline Session G & G1-G6 \\
\hline Session H & H1-H6
\end{tabular}

Saturday, April 9, 2011

\begin{tabular}{|lr|}
\hline Session I & I1-I4 \\
\hline Session J & J1-J4 \\
\hline Session K & K1-K4 \\
\hline Session L & L1-L4 \\
\hline
\end{tabular}

\section{POSTER PRESENTATIONS}

Thursday, April 7 and Friday, April 8, 2011

P1-P66

STUDENT POSTER PRESENTATIONS

Thursday, April 7 and Friday, April 8, 2011

SP1-SP31

\section{CLINICAL VIGNETTE POSTERS}

Thursday, April 7 and Friday, April 8, 2011

CVP1-CVP3

\section{AUTHOR INDEX}


ORAL PRESENTATIONS

THURSDAY, APRIL 7

ROOM: VILLE-MARIE A

\section{SESSION A}

A1

\section{A PROFESSIONAL AND PUBLIC AWARENESS CAMPAIGN TO PROMOTE THE PRUDENT USE OF ANTIBIOTICS: \\ ANTIBIOTIC AWARENESS DAY CANADA 2010 \\ K BUNZELUK ${ }^{1}$, L SAXINGER ${ }^{2}$, K LARYEA ${ }^{3}$, G LOVAGI ${ }^{4}$, G HANSEN ${ }^{5}$, R BARCLAY ${ }^{1}$, T DONALDSON ${ }^{3}$, M FAST ${ }^{1}$, A FAGAN ${ }^{3}$, E HYDESMITH ${ }^{1}$ ${ }^{1}$ National Collaborating Centre for Infectious Diseases, Winnipeg, MB; ${ }^{2}$ AMMI Canada Stewardship and Resistance Committee/ University of Alberta, Edmonton, AB; ${ }^{3}$ Public Health Agency of Canada; ${ }^{4}$ Association of Medical Microbiology and Infectious Disease (AMMI) Canada, Ottawa, ON; ${ }^{5}$ Community and Hospital Infection Control Association, Winnipeg, MB}

OBJECTIVE: In November 2010, six Canadian health partners joined to recognize Antibiotic Awareness Day in Canada, coinciding with similar events in Europe and the United States. The purpose of the one-day campaign was to help combat the emergence and spread of antibiotic-resistant bacteria by promoting the prudent use of antibiotics, increasing awareness, and providing tools for health practitioners and patients.

METHODS: One of the key steps in planning Antibiotic Awareness Day was to bring together partners that could contribute expertise and communicate key messages. Six Canadian organizations and two international agencies formally participated in the event, along with numerous informal partners and individual experts. Activities and materials focused on the interaction between patients and health practitioners.

RESULTS: A number of tools were developed for Antibiotic Awareness Day including: "prescription pad" for symptomatic treatment of viral illnesses, sample dialogue text for doctors, frequently asked questions, information for patients and parents, and information sessions for professionals through a webinar series and colloquium. Numerous communication methods were also used, including: bilingual website (www.AntibioticAwareness.ca), media release, electronic newsletters, links from partner websites, and material shared through membership lists. As intended, the "pilot" campaign was recognized more by health professionals than the public, with the webinars deemed of particular value.

CONCLUSIONS: Awareness and education programs must be integrated, collaborative and ongoing. The impact of Canada's first Antibiotic Awareness Day will be reviewed and built upon in upcoming years. The review will also be used to inform national activities for the WHO's World Health Day in April 2011, which will focus on the global spread of antimicrobial resistance.

\section{A2}

\section{CHANGES IN ANTIBIOTIC UTILIZATION FOR ACUTE OTITIS MEDIA AMONG CHILDREN}

F MARRA $^{1,2}{ }^{2}$ D PATRICK ${ }^{1,2}$, M CHONG $^{2}$, R MCKAY $^{2}$, E BLONDEL-HILL $^{3}$, K DREHER $^{2}$, W BOWIE ${ }^{1}$

${ }^{1}$ University of British Columbia; ${ }^{2} \mathrm{BC}$ Centre for Disease Control; ${ }^{3}$ Interior Health Authority, Vancouver, BC

OBJECTIVES: Do Bugs Need Drugs (DBND) is a multifaceted antimicrobial stewardship program which was implemented in British Columbia in 2005 to minimize inappropriate use of antibiotics within the population. We describe a time series analysis of changes prescribing for acute otitis media (AOM) associated with the program.

METHODS: Prescription data tracked by PharmaNet were anonymously linked to the Medical Services Plan (MSP) to determine prescriptions associated with AOM from 1998 to 2008. We evaluated the two main classes used for treatment of $\mathrm{AOM}$ - pencillins and macrolides. Data were stratified according to children $<2$ years, $2-5$ years, and $6-14$ years of age. To study changes associated with the DBND program, monthly rates were seasonally adjusted and then fitted to segmented linear regression models, controlling for autoregressive effects. The main output measure is the relative change in trend pre- (before October 2005) and post-intervention (after October 2005), where a negative number reflects a reduction in utilization rate at the end of 2008 compared to the predicted rate at that time had the DBND program not been implemented.

RESULTS: For all children, the relative change for penicillins was $2.8 \%$ (95\% CI: $-5.6 \%, 11.9 \%)$; for macrolides was $-41.1 \%(-45.8 \%,-36.3 \%)$; for azithromycin was $-86.0 \%(-88.4 \%,-83.2 \%)$ and for clarithromycin was $-48.6 \%(-53.0 \%,-44.2 \%)$. For children under 2 years of age, the relative change for penicillins was $22.2 \%(9.2 \%, 35.1 \%)$; for macrolides was $-42.6 \%(-48.3 \%,-36.9 \%)$; for azithromycin was $-85.8 \%(-88.4 \%$, $-82.5 \%)$ and for clarithromycin was $-49.0 \%(-55.3 \%$ to $-42.7 \%)$. Similar trends were seen in children between the ages of $2-5$ years, and 6-14 years.

CONCLUSIONS: Utilization of penicillins for AOM stabilized while that for second generation macrolides decreased in association with implementation of the DBND program. These observations most likely reflect a longer term decline associated with physicians opting for the "watch and wait" approach and the impact of the program and other factors in moving prescriptions to narrower spectrum first line agents when they are issued.

\section{A3 \\ ANTIBIOTIC UTILIZATION FOR UPPER RESPIRATORY INFECTION: REVELATIONS FROM TIME SERIES ANALYSIS IN THE ERA OF THE DO BUGS NEED DRUGS? (DBND) PROGRAM}

D PATRICK ${ }^{1,2}$, F MARRA $^{1,2}$, M CHONG $^{2}$, R MCKAY $^{2}$, K DREHER $^{2}$, W BOWIE ${ }^{1}$, E BLONDEL-HILL ${ }^{1,3}$

${ }^{1}$ University of British Columbia; ${ }^{2} \mathrm{BC}$ Centre for Disease Control, Vancouver; ${ }^{3}$ Interior Health Authority, Kelowna, BC

OBJECTIVES: Because community antibiotic stewardship efforts have focused on prescribing for upper respiratory infection (URI), we sought to study trends in antibiotic utilization for URI indications and assess changes associated with DBND program introduction in British Columbia in October, 2005 through time series analysis.

METHODS: Prescription data from BC PharmaNet were anonymously linked to associated Medical Services Plan (MSP) codes to quantify prescriptions associated with upper respiratory infection from 1998 to 2008. We studied children $\leq 14$ years of age (expressed as prescription rates) and people $\geq 15$ years of age (expressed as utilization rate in defined daily doses according to the WHO Anatomical Therapeutic Chemical system). To study changes associated with the DBND program, monthly rates were seasonally adjusted and then fitted to segmented linear regression models, controlling for autoregressive effects. The main output measure is the relative change in trend, where a negative number reflects a reduction in utilization rate at the end of 2008 compared to the predicted rate at that time had the DBND program not been implemented.

\section{RESULTS:}

\begin{tabular}{lcc} 
& \multicolumn{2}{c}{ Relative Change in Trend (95\% Confidence Interval) } \\
\cline { 2 - 3 } & People aged $\geq \mathbf{1 5}$ years & Children aged $\leq 14$ years \\
\hline All Antibiotics & $-6.2 \%(-12.3 \%$ to $-0.1 \%)$ & $-18.2 \%(-27.8 \%$ to $-8.7 \%)$ \\
Penicillins & $-8.1 \%(-13.5 \%$ to $-2.7 \%)$ & $-12.7 \%(-22.8 \%$ to $-2.7 \%)$ \\
Macrolides & $-12.0 \%(-17.8 \%$ to $-6.1 \%)$ & $-32.2 \%(-40.1 \%$ to $-24.3 \%)$ \\
Azithromycin & $-69.2 \%(-72.6 \%$ to $-65.2 \%)$ & $-86.6 \%(-89.3 \%$ to $-83.3 \%)$ \\
Clarithromycin & $-22.7 \%(-27.2 \%$ to $-18.2 \%)$ & $-48.7 \%(-54.2 \%$ to $-43.1 \%)$ \\
\hline
\end{tabular}

Similar improvements in trend were seen for specific URI diagnoses such as bronchitis and pharyngitis (data not shown)

CONCLUSIONS: Favourable changes against predicted utilization trends have been seen in association with DBND program. Future models for URI will be refined by adjustment for influenza-like illness activity. 
A4

METHICILLIN-RESISTANT STAPHYLOCOCCUS AUREUS (MRSA) BLOODSTREAM INFECTIONS (BSIS) IN CANADIAN HOSPITALS FROM 1995 TO 2009

A MOUNCHILI ${ }^{1}$, A SIMOR ${ }^{1,2}$, D GRAVEL ${ }^{1}$, M MULVEY $^{3}$, E BRYCE ${ }^{4}$, A MATLOW $^{5}$, M LOEB ${ }^{6}$, AJ MCGEER ${ }^{7}$; Canadian Nosocomial Infection Surveillance Program ${ }^{1}$

${ }^{1}$ Centre for Communicable Diseases and Infection Control, Public Health Agency of Canada, Ottawa; ${ }^{2}$ Sunnybrook Health Sciences Centre, Toronto, ON; ${ }^{3}$ National Microbiology Laboratory, Public Health Agency of Canada, Winnipeg, MB; ${ }^{4}$ Vancouver General Hospital, Vancouver, BC; ${ }^{5}$ The Hospital for Sick Children, Toronto; ${ }^{6}$ Hamilton Health Sciences Corporation, Hamilton; ${ }^{7}$ Mount Sinai Hospital, Toronto, ON

OBJECTIVES: The purpose of this study was to describe the epidemiology of MRSA BSIs in acute care centres participating in the Canadian Nosocomial Infection Surveillance Program (CNISP) from 1995 to 2009.

METHODS: A prospective laboratory-based surveillance of incident cases of MRSA was conducted from 1995 to 2009 in CNISP hospitals across the country. Upon identification of a MRSA BSI, the patient's medical record was reviewed to collect clinical and epidemiologic data. BSI was diagnosed when MRSA was isolated from a blood culture. Random subsets (1995 to 2007) of blood culture isolates or all of them (2008-2009) were further characterized using pulsed-field gel electrophoresis (PFGE).

RESULTS: From 1995 to 2009, the number of cases of MRSA BSIs diagnosed annually rose exponentially from less than 20 cases to over 350, corresponding to an incidence rate of 0.02 per 1,000 admissions in 1995 and 0.54 in 2009. Hospitals in Atlantic Canada had the lowest rates compared to the rest of the country, except from 2005 and 2008 when Central Canada rates were lower. Pediatric hospitals had lower MRSA BSI rates than those in either adult or mixed adult/pediatric hospitals. Since 2001, the most common strain isolated from bacteremic patients was CMRSA2, followed by CMRSA10, a community-associated clone, that has emerged since 2005 as the second most common strain.

CONCLUSIONS: MRSA BSI rates have increased over time in CNISP hospitals. CMRSA2, accounting for over $50 \%$ of all the cases, has been predominant; followed by CMRSA10, a community-associated strain.

\section{A5}

\section{IMPACT OF CA-MRSA ON POPULATION-BASED ANTIMICROBIAL USE FOR SKIN AND SOFT TISSUE INFECTIONS}

\section{FMARRA $^{1,2}$, D PATRICK ${ }^{1,2}$, M CHONG $^{2}$, R MCKAY $^{2}$, W BOWIE $^{1}$}

${ }^{1}$ University of British Columbia; ${ }^{2} \mathrm{BC}$ Centre for Disease Control, Vancouver, BC

OBJECTIVES: Community acquired methicillin resistant Staphylococcus aureus (CA-MRSA) has been a rapidly growing problem for much of the past decade. We have previously demonstrated an associated increase in physician visits for skin and soft tissue infections (SSTI). We sought to examine associated changes in antibiotic prescribing for SSTI 1998-2008. METHODS: Prescription data from BC PharmaNet were anonymously linked to associated MSP codes to quantify prescriptions associated with SSTI from 1998 to 2008. Prescriptions were expressed as their defined daily dose (DDD) per 1000 inhabitants per day according to the WHO Anatomical Therapeutic Chemical system. Overall and class-specific rates of consumption were described by year for treatment of skin and soft tissue infections.

RESULTS: Between 1996 and 2008, the BC rate for all antibiotics used for treatment of skin and soft tissue infections increased by $50 \%$ from 0.42 DDD per 1000 inhabitant-days to 0.63 DDD per 1000 inhabitant-days. The majority of this increase was seen with use of trimethoprim/sulfamethoxazole (TMP/SMX) which increased 4.8-fold. Use of cephalosporins (2.6-fold) and macrolides/lincosamides ( 1.6 fold) also increased while tetracyclines and penicillins remained steady over time. The increase seen with the macrolides/lincosamide class was primarily related to an increase in the use of clindamycin ( 7.3 fold).

\begin{tabular}{lll} 
Drug & $\mathbf{1 9 9 8}$ & $\mathbf{2 0 0 8}$ \\
\hline Penicillins & 0.18 & 0.12 \\
Cephalosporins & 0.11 & 0.30 \\
Macrolides/lincosamides & 0.05 & 0.08 \\
Tetracyclines & 0.037 & 0.045 \\
Trimethoprim/Sulfamethoxazole & 0.01 & 0.048 \\
\hline
\end{tabular}

CONCLUSIONS: Antibiotic use for skin and soft tissue infections has increased over time in BC. While much of the increase reflects an appropriate change to TMP/SMX given prevalence of CA-MRSA, cephalosporin and clindamycin use has also been driven up.

\section{A6}

\section{COMMUNITY AND HOSPITAL ANTIBIOTIC PROFILES OF GROUP B STREPTOCOCCUS FOR PENICILLIN, ERYTHROMYCIN AND CLINDAMYCIN IN VANCOUVER, CANADA}

I WONG ${ }^{1}$, S DHALIWAL ${ }^{2}$, G AL-RAWAHI ${ }^{1}$, C PIENAAR ${ }^{1,3}$, M KELLY $^{1,3}$, L MARTINEZ ${ }^{3}$, R REYES ${ }^{1,3}$

${ }^{1}$ Department of Pathology and Laboratory Medicine, University of British Columbia; ${ }^{2}$ Division of Medical Microbiology, Department of Pathology and Laboratory Medicine, BC Children's Hospital and Women's Health Centre; ${ }^{3}$ LifeLabs Medical Laboratory Services, Vancouver, BC

OBJECTIVE: Intrapartum chemoprophylaxis for Group B Streptococcus (GBS) colonization decreases GBS neonatal sepsis mortality. American data have demonstrated resistance rates as high as $32 \%$ for erythromycin and $20 \%$ for clindamycin. The purpose of this study was to determine the susceptibility patterns of penicillin, clindamycin, and erythromycin for GBS in Vancouver, Canada.

METHODS: Consecutive GBS-screening samples were collected from LifeLabs Medical Services, a large community laboratory, and BC Children's and Women's Health Center (BCCWH) from April through November, 2010. Standard microbiology methods, including Prolex kits were used for GBS identification. Penicillin Etest MIC, disk diffusion for erythromycin and clindamycin and D-test were performed and interpreted according to CLSI M100-S20. The susceptibility patterns from both centers were compared.

RESULTS: All BCCWH ( $\mathrm{n}=118)$ isolates were penicillin susceptible, with a mean MIC $(\mu \mathrm{g} / \mathrm{mL})$ of 0.060 , median MIC of 0.064 , and $\mathrm{MIC}_{90}$ of 0.064 . At LifeLabs $(n=163)$, one isolate had a penicillin MIC of 0.125 ; mean MIC was 0.064, median MIC was 0.064, and $\mathrm{MIC}_{90}$ was 0.094 . Erythromycin/clindamycin resistance rates were $26.3 \% / 3.7 \%$ at BCCWH and $20.9 \% / 16.0 \%$ at LifeLabs. Inducible clindamycin resistance was $6.8 \%$ at BCCWH and $5.5 \%$ at LifeLabs.

CONCLUSIONS: GBS continue to be susceptible to penicillin. One community isolate had a penicillin MIC of $0.125 \mu \mathrm{g} / \mathrm{mL}$. The high erythromycin and clindamycin resistance rates found are consistent with literature. Although resistance rates were higher at the hospital center, there were no statistically significant differences.

\section{ROOM: ST-ANTOINE A}

\section{SESSION B}

\section{B1}

\section{PERFORMANCE AND WORKFLOW COMPARISON OF CERVISTA $^{\circledR}$ HPV HR AND HC2 ON SUREPATH LIQUID- BASED PAP SPECIMENS}

I KAPALA ${ }^{1}$, N HARROUN ${ }^{1}$, D JANG ${ }^{2}$, K BIERS $^{1}$, J GILCHRIST ${ }^{2}$, R TOOR $^{2}$, J SUMNER ${ }^{1}, M^{1}$ CHERNESKY ${ }^{2}$

${ }^{1}$ Gamma Dynacare Medical Laboratories, Brampton; ${ }^{2}$ McMaster University/St Joseph's Healthcare, Hamilton, ON

OBJECTIVE: To compare the Cervista HPV HR test to HC2 in a community diagnostic laboratory to determine assay agreement and workflow. 
METHODS: Fresh media was added to SurePath L-Pap samples to $3.5 \mathrm{~mL}$ then aliquoted as follows: HC2 (Qiagen), 2mL; Cervista (Hologic), 1mL; and Linear Array (LA) (Roche), $0.25 \mathrm{~mL}$. Samples $(\mathrm{n}=264)$ were extracted and tested according to package inserts with modifications for manual extraction. Outcomes for $\mathrm{HC} 2$ were pos (RLU/CO>3), neg $(<1)$ or indeterm (1-3). Cervista results were based on analysis of 3 separate wells. Positives had \%CV genomic DNA (gDNA) $<25 \%$, one or more HPV FOZ $>0.70$, and HPV FOZ ratio $\geq 1.525$, negative, HPV FOZ $<1.525$ and indeterm had low gDNA, high \%CV and low HPV FOZ and were retested. Discordants were tested by LA. Total and hands-on time for each assay were recorded.

RESULTS: The assays agreed on 90 positives $(82.6 \%)$ and 116 negatives $(84.7 \%)$ for an overall agreement of $90.7 \%(\mathrm{~K}=0.81)$. LA detected HR HPV genotypes in 11 of 19 samples which were HC2+/Cervista-. Reported testing time was $9.25 \mathrm{hrs}$ for Cervista with $5.25 \mathrm{hrs}$ hands-on compared to $6.25 \mathrm{hrs}$ with $4 \mathrm{hrs}$ hands-on for HC2.

CONCLUSION: SurePath samples showed indeterminate results in the Cervista HR HPV assay due to inconsistent sample extraction, DNA inhibition or contamination. A substantial number of Cervista specimens contained HR HPV by LA. Cervista walk-away time was 4 hrs but required 1.25 hrs more hands-on time than $\mathrm{HC} 2$ and the total assay time was longer. Both assays may benefit from automated extraction.

\section{B2}

COMPARISON OF THE ROCHE COBAS ${ }^{\circledR} 4800$ VS QIAGEN HC2 ${ }^{\circledR}$ TESTS FOR DETECTION OF HIGH-RISK HPV DNA $\mathrm{K} \mathrm{CEBALLOS}^{1,2}$, D VAN NIEKERK ${ }^{1,2}$, W MEI ${ }^{3}$, D COOK ${ }^{3}$, R SHOW ${ }^{3}$, L SMITH $^{2}$, E FRANCO ${ }^{4}$, G OGILVIE ${ }^{1,3}$, A COLDMAN ${ }^{1,2}$, M KRAJDEN ${ }^{1,3}$

${ }^{1}$ University of British Columbia; ${ }^{2} \mathrm{BC}$ Cancer Agency; ${ }^{3} \mathrm{BC}$ Centre for Disease Control, Vancouver, BC; ${ }^{4}$ McGill University, Montreal, QC OBJECTIVE: To compare the performance of the Roche COBAS $4800^{\circledR}$ vs. Qiagen hc2 ${ }^{\circledR}$ tests for detection of high-risk (HR) HPV DNA.

METHODS: ThinPrep ${ }^{\circledR}$ cervical specimens from females enrolled in the HPV FOCAL Trial (ISRCTN79347302) were tested for HR HPV by Qiagen hc $2{ }^{\circledR}$ High Risk HPV DNA Test (HC2) and Roche cobas ${ }^{\circledR} 4800$ HPV (COBAS). All specimens were also tested by Roche Linear Array HPV Genotyping Test (LA).

RESULTS: For 2586 specimens, overall agreement between $\mathrm{HC} 2$ and COBAS was $95.9 \%(\kappa=0.73 ; 95 \%$ CI $0.68-0.78)$. HC2 and COBAS were both positive for 160 specimens; by LA, 156/160 were HR HPV positive, 1/160 was low risk (LR) HPV (HPV89) and 3/160 were HPV negative. Of $2321 \mathrm{HC} 2 / \mathrm{COBAS}$ negative specimens, by LA, 2188 were negative or had only LR HPV and 33 were HR HPV positive [6 HPV 16, 4 HPV 18 and 23 other HR HPV (non-HPV 16 or 18)]. Of the 105 specimens with discordant $\mathrm{HC} 2 / \mathrm{COBAS}$ results, 53/105 were $\mathrm{HC2}$ positive/COBAS negative and 52/105 were $\mathrm{HC} 2$ negative/COBAS positive (Table 1). Test signals for most discordant specimens were close to the respective assay detection threshold.

CONCLUSIONS: Agreement between $\mathrm{HC} 2$ and COBAS was high. Based on LA as a reference standard, HC2 had more false positive HR HPV detections than COBAS, and COBAS detected more HR HPV, including HPV 16 and 18, than HC2.

\section{Table 1}

Linear Array Results for Discordant HC2/COBAS Specimens

\begin{tabular}{lccccc}
\hline & & HPV & \multicolumn{3}{c}{ HR HPV } \\
& N & Negative & LR HPV & (non 16,18) HPV 16, 18 \\
\hline HC2+/COBAS- & 53 & 27 & 22 & 4 & 0 \\
HC2-/COBAS+ & 52 & 10 & 4 & 25 & 13 \\
\hline
\end{tabular}

\section{B3}

\section{MODIFIED TRIPLEX QUANTITATIVE PCR ASSAY FOR HUMAN POLYOMAVIRUSES JC AND BK \\ V GOLESKI, T DUMONCEAUX, A SEVERINI}

National Microbiology Laboratory, Winnipeg, MB

OBJECTIVE: We have modified our original triplex TaqMan-based qPCR assay for the human polyomaviruses BK and JC (Dumonceaux et al, 2008, J Clin Microbiol;46:2829-36). The modified assay is now able to detect a BK variant that is circulating in approximately $5 \%$ of cases. The objective of this study is to detect the mutant BK and show that there is no significant loss of sensitivity or specificity compared with the old triplex method.

METHODS: A new forward primer and probe were designed with the mutations harboured in the BK mutant. The new primer and probe were added to the assay at several concentrations and the specificity, sensitivity and reproducibility were tested. PCR conditions and other reagents were left unchanged. A validation was performed testing 60 samples.

RESULTS: Validation against the previously published triplex assay showed that there is no significant difference between the two assays with regards to sensitivity and specificity. BK results and JC results were treated as separate results. Of the 30 previously positive JC samples all 30 were positive with the new method. Of the 30 previously positive BK samples all 30 were positive with the new method. The new assay detected an additional BK positive sample which is likely due to the mutant BK virus that was missed using the originally described method.

CONCLUSIONS: Evaluation of the 60 previously tested samples with the modified triplex assay shows that this method is proficient for the detection of the wild type and mutant $\mathrm{BK}$ and JC polyomaviruses.

\section{B4}

DEVELOPMENT AND EVALUATION OF A REAL TIME RT-PCR ASSAY FOR SPECIFIC DETECTION OF ENTEROVIRUSES ON A VARIETY OF SPECIMEN TYPES

S WONG ${ }^{1}$, K PABBARAJU $^{1}$, KL GILL $^{1}$, R TELLIER ${ }^{1,2}$

${ }^{1}$ Provincial Laboratory For Public Health (Microbiology);

${ }^{2}$ Department of Microbiology, Immunology and Infectious

Diseases, University of Calgary, Calgary, AB

OBJECTIVE: Enterovirus is a genus within the family Picornaviridae that includes important human pathogens; more than 100 serotypes are identified thus far, grouped into 4 species A to D. They cause a variety of diseases including enanthema and exanthema, respiratory diseases, myocarditis and meningitis. Recombination and sequence drift are common among enteroviruses $\left(E V_{s}\right)$, making it challenging to design an assay that can detect all EVs without cross-detection of viruses within the closely related genera Rhinovirus and Parechovirus. This study aimed to develop a comprehensive and enterovirus-specific real-time nucleic acid amplification assay.

METHODS: The assay targets the 5'-UTR region of the EV genome; detection was performed using a hydrolysis probe labelled with FAM and a minor groove binding protein at the 3'-end. Detection of $\mathrm{EV}_{\mathrm{s}}$ was confirmed using isolates of human EV species A (4), B (31), C (7) and D (1). Specificity was evaluated using samples containing high copy numbers of rhinovirus clades $\mathrm{A}$ and $\mathrm{B}$, parechoviruses type 1 and 2 , and a panel of respiratory viruses and bacteria. CSF (63), plasma/serum (8), amniotic fluid (1), throat/nasopharyngeal swab (9), feces (7), and swabs (8) collected from symptomatic patients that tested positive by the assay currently used in our laboratory were retrospectively tested with the new assay. Samples positive for rhinoviruses A, B and C groups ( $\mathrm{n}=69$ ) were also tested.

RESULTS AND CONCLUSION: The sensitivity of the new assay for in-vitro transcribed RNA of CVA9, CVA16, CVB3 and enterovirus 70 was approximately 5, 50, 5 and 5 copies per reaction, respectively. The new assay could detect all EV serotypes available in our lab and did not cross react with high copy templates of other viruses and bacteria tested. All rhinoviruses and parechoviruses tested were not detected. The new assay was $100 \%$ concordant for the detection of retrospective positives. This assay will lead to an improved diagnosis and surveillance of EV, including the recently characterized serotypes (based on in-silico analysis). 


\section{B5}

COMPARISON OF WESTERN BLOT AND INNO-LIA FOR SEROLOGICAL HUMAN IMMUNODEFICIENCY VIRUS (HIV) DIAGNOSIS

K KADIVAR, J KIM

Public Health Agency of Canada, Ottawa, ON

OBJECTIVE: Currently the Western Blot (Wb) is the gold standard for confirmatory testing of anti-HIV antibodies. However, indeterminate results are common and can lead to false positive diagnosis. Indeterminate status leads not only to a state of uncertainty and stress but also delays necessary treatment in the case of true infection. A comparison of BIORAD's Genetic Systems HIV-1 Western blot assay and Innogenetics' INNO-LIA HIV I/II Score was used to assess the possibility of introducing LIA as a potential replacement for Wb.

METHODS: Samples tested by the National Lab for HIV Reference Services (NLHRS) serological testing algorithm (2002 to 2009) in which a final diagnosis was generated were reviewed. The NLHRS algorithm includes a combination of the Wb, LIA and RIPA. Specifically, results from the $\mathrm{Wb}$ and LIA were compared $(\mathrm{n}=1212)$. The majority of samples received were either plasma or sera, molecular testing by PCR was conducted on only a limited number of samples.

RESULTS: Sensitivity was comparable between the LIA and Wb. LIA was able to detect antibody from $108(100 \%)$ positive samples while Wb was able to detect 105 (97.2\%). The major difference between the assays was observed in the specificity. Of the 1104 negative samples, 1043 (94.5\%) were found negative by LIA and only 691 (62.6\%) were negative by Wb. The negative predictive value (NPV) was $100 \%$ for LIA and $99.6 \%$ for $\mathrm{Wb}$. Major difference were observed in the positive predictive value (PPV) which was $63.9 \%$ for LIA and $20.3 \%$ for $\mathrm{Wb}$.

CONCLUSION: Based on the results, the LIA performed favorably compared to the $\mathrm{Wb}$ due to the reduced number of false reactive results. LIA can eliminate the need for repeat testing and reduce costs. More importantly it would allow for quicker and more accurate diagnosis for clinical HIV testing.

\section{B6}

EXTERNAL QUALITY ASSESSMENT (EQA) OF INFLUENZA (FLU) AND RESPIRATORY SYNCYTIAL (RSV) VIRUSES USING SIMULATED SPECIMENS FOR DIRECT DETECTION METHODS

M COUILLARD ${ }^{1}$, R LAMIRANDE ${ }^{1}$, P TURCOTTE ${ }^{1}$, H CHAREST ${ }^{1}$, C BÉLIVEAU $^{2}$, A-M BOURGAULT ${ }^{1}$

${ }^{1}$ Laboratoire de santé publique, Institut national de santé publique du Québec, Sainte-Anne-de-Bellevue; ${ }^{2}$ Hôpital MaisonneuveRosemont, Montréal, QC

OBJECTIVES: To develop and evaluate simulated respiratory specimens suitable for either culture, nucleic acid amplification test (NAAT), rapid tests (RT) or direct immunofluorescence (DFA) in order to monitor the performance of clinical laboratories for the detection of Flu and RSV.

METHODS: Simulated specimens were prepared by mixing infected and non infected cell suspensions with PBS and albumin, and adjusted to allow the detection of positive specimens by all methods after 48 hours. Two panels of three specimens ( 2 positives and 1 negative) were sent by courier.

RESULTS: A total of 82 laboratories tested the two panels. The majority (78/82 in 02/2009 and 68/82 in 02/2010) used RT Flu assays. The global performance was $>97 \%$ for both panels. The table presents the ratios of accurate results/total number of expected results:

\begin{tabular}{lcccc} 
& Flu A (2009) & Flu B (2009) & Flu A \& B (2010) & RSV (2010) \\
\hline Culture & $15 / 16$ & $16 / 16$ & $17 / 18$ & $14 / 15$ \\
NAAT & $9 / 9$ & $9 / 9$ & $26 / 27$ & $9 / 9$ \\
RT & $228 / 234$ & $233 / 234$ & $203 / 204$ & $89 / 90$ \\
DFA & $9 / 9$ & $9 / 9$ & $6 / 6$ & $6 / 6$ \\
\hline
\end{tabular}

All positive specimens (Flu A, Flu B and RSV) were stable and adequate for $E Q A$ no matter the detection methods used by the different laboratories
CONCLUSION: The development of "universal" simulated specimens for EQA is feasible, convenient and efficient to monitor the quality of test results for Flu and RSV obtained by different methods.

\section{B7}

PILOT STUDY OF DENGUE IN TEGUCIGALPA, HONDURAS

I SRIGLEY ${ }^{1}$, K HAMILTON ${ }^{1}$, S ESKANDARIAN ${ }^{1}$, L CHILDERHOSE ${ }^{1}$, E FERNANDEZ ${ }^{2}$, I LORENZANA ${ }^{3}$, M LOEB LO $^{1,2,4,5}$

${ }^{1}$ Department of Pathology and Molecular Medicine; ${ }^{2}$ Department of Clinical Epidemiology and Biostatistics, McMaster University, Hamilton, ON; ${ }^{3}$ National Microbiology Laboratory, Tegucigalpa, Honduras; ${ }^{4}$ Department of Medicine; ${ }^{5}$ Michael G DeGroote Institute for Infectious Disease Research, McMaster Unviersity, Hamilton, ON

OBJECTIVES: Dengue is a mosquito-borne virus that is found in tropical and sub-tropical regions worldwide. It is estimated that two-fifths of the world's population is at risk from dengue. The Caribbean and Latin America have experienced a significant increase in dengue over the past 30 years, with Honduras having one of the highest incidence rates in the region. The objectives of this study were to establish the feasibility of conducting a cohort study to determine seroconversion and risk factors for dengue in Honduran neighbourhoods.

METHODS: A population-based survey was performed in the Villa Adela neighbourhood of Tegucigalpa between June and December 2008. All participants $(n=234)$ were visited at baseline, midpoint, and end of the study period by a 10 person field team to obtain demographic data and blood specimens for serology. Twice-weekly surveillance was performed for dengue fever symptoms, and suspected cases were investigated with dengue virus PCR. All serum samples were tested for the presence of anti-dengue $\operatorname{IgG}$ antibodies using a commercially available indirect ELISA kit (Inverness Medical, Australia).

RESULTS: A total of 604 specimens were collected from 235 participants. By the end of the study period, $92.1 \%$ (163/177) of participants were positive for anti-dengue IgG antibodies. Nine patients reported symptoms of possible dengue fever during the study period, but all were negative by PCR. Two participants seroconverted, neither of whom experienced any symptoms of dengue fever.

CONCLUSIONS: It is feasible to conduct large cohort studies of dengue in highly exposed Tegucigalpa neighborhoods.

\section{ROOM: VILLE-MARIE B}

\section{SESSION C}

\section{C1}

PERFORMANCE COMPARISON OF ONTARIO VERSUS U.S. LABORATORIES ON ENTERIC PARASITOLOGY EXTERNAL QUALITY ASSESSMENT (EQA)/PROFICIENCY TESTING (PT) SURVEYS

CA FLEMING, A SARABIA, M DESJARDINS, F JAMIESON, K KATZ, H MEANEY, SM POUTANEN, D RAYMONDO, J GUN-MUNRO, G FLYNN

Quality Management Program - Laboratory Services, Toronto, ON OBJECTIVES: To compare performance between Ontario and U.S. labs on enteric parasitology EQA/PT surveys and to assess whether a significant decrease in the number of labs performing parasitology has resulted in improved overall performance.

METHODS: A retrospective review of performance by Ontario labs on QMP-LS surveys conducted between 2003 and 2008 that used actual patient samples requiring labs to prepare and examine a concentrate and stained smear was compared with performance data for U.S. labs from a variety of PT schemes for the same period. Most U.S. schemes provided a concentrate, prepared smear for staining and/or photos. A review of performance data for Ontario labs from 1995 to 2010 was also carried out to determine if any trends in performance related to a decrease in the number of parasitology labs could be identified. 
RESULTS: The data for 2003-2008 showed that with the exception of Endolimax nana, $>80 \%$ of Ontario labs correctly identified the 18 parasite species for which comparable data were available $100 \%$ of the time compared with U.S. labs which did so, on average, $63 \%$ of the time (range $15 \%-93 \%)$. Performance data for identification of 29 different parasite species on QMP-LS surveys for 1995-2010 were as follows:

Year $\quad 1995199619971998199920002001200220032004200520062007200820092010$ \begin{tabular}{lllllllllllllllll}
\hline No. labs & 66 & 55 & 46 & 36 & 33 & 30 & 29 & 27 & 27 & 24 & 19 & 19 & 18 & 17 & 16 & 16
\end{tabular} \begin{tabular}{lllllllllllllllll} 
Score (\%) & 96 & 93 & 94 & 95 & 94 & 89 & 86 & 85 & 85 & 91 & 89 & 90 & 94 & 97 & 94 & 95 \\
\hline
\end{tabular}

CONCLUSIONS: Ontario labs significantly outperformed their U.S. counterparts in their respective EQA/PT programs. Clarification of the reasons behind performance differences would be beneficial to inform future planning. Performance by Ontario labs on QMP-LS parasitology surveys did not change significantly over the 16 years despite the fact that the number of labs decreased from 66 to 16 during this time.

\section{C2}

PRACTICAL BENCH COMPARISON OF 3 CHROMOGENIC AND 2 STANDARD URINE CULTURE MEDIA FOR DETECTION AND IDENTIFICATION OF URINARY TRACT PATHOGENS

P KORNHERR ${ }^{1}$, D DOWHANIUK ${ }^{1}, \mathrm{C} \mathrm{GHOSH}^{2}$, M GUZMAN $^{2}$, L LEWIS $^{2}$, R PIZARRO ${ }^{1}$, J KAPALA ${ }^{2}$

${ }^{1}$ Gamma-Dynacare Medical Laboratories, Ottawa; ${ }^{2}$ Gamma-Dynacare Medical Laboratories, Toronto, ON

INTRODUCTION: This survey compared the performance of 3 recent versions of chromogenic plates (Uri-Select-Bio-Rad "S", Orientation-BD, "O", UTI-Oxoid "U") with 2 standard media (CLED-Bio Media C, BA/ Mac biplate B/M-bioMerieux) in regards to incubation times, identification (ID), recovery rates, "spot" testability, ease of use and cost.

METHODS: A) 381 positive patient urines with 20 different pathogenic species (previously characterized) were inoculated in duplicate onto $\mathrm{S}, \mathrm{O}, \mathrm{U}$ plus $\mathrm{C}$ in one center and $\mathrm{B} / \mathrm{M}$ in the other. Plates were judged on colony discernibility for work-up, chromogenic and/or standard identifications, and colony counts, with respect to readings at 14-16, 18, 24 hrs incubation.

B) 100 known "contaminated" urines were inoculated as above. Numbers of isolates with their ease of differentiation were evaluated at 14,16, $24 \mathrm{hrs}$. RESULTS: A) At $14-16$ hrs. $95 \%$ of E.coli on S,O,U,C,B/M and $100 \%$ Enterococci on S,O,U,B/M were discernable and easily identifiable. O verified for ID of these. S,U gave similar results. B/M grew all at 14-16 hrs except for 1 of 2 C. urealyticum and 1 yeast. P mirabilis, Citrobacter sp, S saprophyticus performance varied the most with manufacturers.

DISCUSSION: $15 \%$ of E.coli were NLF but identifiable on O,S,U. O,U and especially $\mathrm{S}$ are superior for mixture differentiation and the ability to identify $80 \%$ of pathogens the same day. They reduce work-up time (up to $18 \%$ ) while reducing ID testing costs (up to $22 \%$ ). At $14-16 \mathrm{hrs}, 95 \%$ of coliforms and Enterococci ( $>90 \%$ pathogens) are discernible on S,O,U,B/ M,C. Gram positives ( $<10 \%$ of pathogens) require $>=18 \mathrm{hrs}$ on $\mathrm{C}, \mathrm{O}, \mathrm{S}, \mathrm{U}$.

C3

DETERMINING PENICILLIN (PEN) AND CEFTRIAXONE (CRO) MIC IN STREPTOCOCCUS PNEUMONIAE (SPN): EVALUATION OF COMMERCIALLY AVAILABLE TREK SENSITITRE BROTH MICRODILUTION (BMD) AND BIOMERIEUX'S ETEST GRADIENT STRIPS USING TWO BRANDS OF MUELLER-HINTON BLOOD AGAR (MHBA) BM WILLEY ${ }^{1}$, K PRAYITNO $^{2}$, T FUNG $^{2}$, A YEBOAH ${ }^{1}$, C LAROCQUE ${ }^{1}$, H NG $^{1}$, K SCHOER $^{1}$, N BERESFORD ${ }^{1}$, P LO ${ }^{1}$, S PONG-PORTER ${ }^{1}$, A) MCGEER ${ }^{1,2}$, SM POUTANEN ${ }^{1,2}$

${ }^{1}$ Mount Sinai Hospital/University Health Network; ${ }^{2}$ University of Toronto, Toronto, ON

OBJECTIVES: Reliable detection of beta-lactam MIC in SPN is critical. CLSI BMD (gold STD) was compared to Sensititre BMD and Etest (ET) on BD and Oxoid MHBA for accuracy in Pen and Cro MIC determination.
METHODS: 200 blood SPN (2000-09) were selected for serotype ( $n=43$ ) and MIC diversity. 0.5 MacFarland suspensions in MHB were inoculated to Trek STP6F panels (incubated and read in ARIS: 20-24h) and to BD and Oxoid MHBA for Pen and Cro ET. After $20 \mathrm{~h}$ in $\mathrm{CO}_{2}$ at $35^{\circ} \mathrm{C}$, ET endpoints were determined by 5 readers. Consensus Pen/Cro ET MIC per agar were compared to ARIS and CLSI MIC.

RESULTS: As per CLSI meningitis (MEN)/non-meningitis (Non-MEN) criteria, $45.5 \% / 3.5 \%$ strains were Pen-R and $19 \% / 10 \%$ Cro-R, respectively, while $20.5 \%$ were Pen-I and $14 \%$ Cro-I by non-MEN criteria. The table below summarizes performance of all methods and media tested; there were no major errors.

\begin{tabular}{|c|c|c|c|c|c|c|}
\hline \multirow{2}{*}{$\begin{array}{l}\text { Penicillin/ } \\
\text { Ceftriaxone }\end{array}$} & \multicolumn{2}{|c|}{ Sensititre STP6F BMD } & \multicolumn{2}{|c|}{ Etest on BD MHBA } & \multicolumn{2}{|c|}{ Etest on Oxoid MHBA } \\
\hline & MEN & Non-MEN & MEN & Non-MEN & MEN & Non-MEN \\
\hline $\begin{array}{l}\% \text { VME } \\
(95 \% \mathrm{Cl})\end{array}$ & $\begin{array}{c}0 / 0 \\
(0-5 / 0-11)\end{array}$ & $\begin{array}{c}0 / 0 \\
(0-40 / 0-32)\end{array}$ & $\begin{array}{c}4 / 3 \\
(1-11 /<0.01-15)\end{array}$ & $\begin{array}{c}57 / 0 \\
(25.84 / 0.32)\end{array}$ & $\begin{array}{c}8 / 16 \\
(4-16 / 7-31) \\
\end{array}$ & $\begin{array}{c}86 / 20 \\
(47.99 .5 / 5.52) \\
\end{array}$ \\
\hline$\% \operatorname{minE}$ & -13 & $9 / 4.5$ & $/ 15$ & $20 / 15$ & $/ 17$ & 19/16 \\
\hline 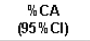 & $\begin{array}{c}100 / 97 \\
(98-100 / 94-99)\end{array}$ & $\begin{array}{c}91 / 96 \\
\text { (86.93/92-98) }\end{array}$ & $\begin{array}{c}98 / 84.5 \\
(95.99 / 78-89)\end{array}$ & $\begin{array}{c}78 / 86 \\
(72.83 / 80.90)\end{array}$ & $\begin{array}{c}96 / 80 \\
(93.98 / 74-85)\end{array}$ & $\begin{array}{c}78 / 83 \\
(72-84 / 77.87)\end{array}$ \\
\hline $\begin{array}{l}\% \pm 1 \text { EA } \\
(95 \% \mathrm{Cl})\end{array}$ & \multicolumn{2}{|c|}{$\begin{array}{c}100 / 100 \\
(98-100 / 98-100)\end{array}$} & \multicolumn{2}{|c|}{$\begin{array}{c}86 / 47 \\
(81.90 / 40-53)\end{array}$} & \multicolumn{2}{|c|}{$\begin{array}{c}60 / 42 \\
(5367 / 3549)\end{array}$} \\
\hline
\end{tabular}

CONCLUSIONS: Compared to CLSI BMD, only STP6F Sensititre performed within acceptable limits as defined by Cumitech 31A. Both Pen and Cro Etest incurred unacceptably high VME, CA and EA on both BD and Oxoid MHBA.

\section{C4}

\section{COMPARISON OF CHROMOGENIC CULTURE METHODS FOR DETECTION OF VANCOMYCIN RESISTANT ENTEROCOCCI (VRE)}

W JANG, G RITCHIE, MG ROMNEY, S CHAMPAGNE, A WONG, J TARUC, J BILAWKA

Providence Health Care, Vancouver, BC

OBJECTIVE: Several new chromogenic culture plates have been marketed for the detection of VRE. We compared the sensitivity, specificity and ease of use of Bio-Rad VRESELECT ${ }^{\mathrm{TM}}$, bioMérieux chromID ${ }^{\mathrm{TM}}$ VRE and Oxoid ${ }^{\circledR}$ Brilliance VRE plates with Oxoid ${ }^{\circledR}$ VRE Selective Broth culture and Oxoid ${ }^{\circledR}$ Bile Esculin Agar plates with $8 \mu \mathrm{g} / \mathrm{mL}$ Vancomycin (BEAV), using a real-time PCR method to confirm the presence of the resistance genes, van $A$ and van $B$, in the cultured bacteria.

METHOD: A total of 500 specimens from screening samples (rectal swab) and stool samples submitted for $\mathrm{C}$ difficile toxin were analyzed. Specimens were suspended in saline, vortexed, cultured on all 5 media, then incubated and read at 1 and 2 days. Confirmatory real-time PCR for vanA and vanB, was performed on positive cultures using an in-house developed, internally controlled PCR reaction. In addition, biochemical identification and vancomycin MIC susceptibility testing was performed on all positive cultures according to manufacturers' instructions.

RESULTS: 61 specimens were confirmed positive for VRE (confirmed by PCR).

\begin{tabular}{|c|c|c|c|c|c|c|c|c|c|c|}
\hline \multirow[b]{2}{*}{ Day } & \multicolumn{2}{|c|}{ VRESelect } & \multicolumn{2}{|c|}{ chromID } & \multicolumn{2}{|c|}{ Brilliance } & \multicolumn{2}{|c|}{ BEAV } & \multicolumn{2}{|c|}{ VRE Broth } \\
\hline & 1 & 2 & 1 & 2 & 1 & 2 & 1 & 2 & 1 & 2 \\
\hline Sens. & $76 \%$ & $90 \%$ & $75 \%$ & $89 \%$ & $80 \%$ & $89 \%$ & $47 \%$ & $84 \%$ & $82 \%$ & $98 \%$ \\
\hline Spec. & $98 \%$ & $97 \%$ & $99 \%$ & $98 \%$ & $98 \%$ & $94 \%$ & $96 \%$ & $92 \%$ & $88 \%$ & $76 \%$ \\
\hline
\end{tabular}

Shaded boxes are the manufacturers' recommended incubation and reading time

CONCLUSION: Oxoid ${ }^{\circledR}$ Brilliance VRE plates and Oxoid ${ }^{\circledR}$ VRE Selective Broth exhibited the best sensitivity when read on the first day. 48 hour incubation resulted in better overall sensitivity for all media with a loss in specificity, more pronounced for the VRE Selective Broth. Bio-Rad VRESELECT ${ }^{\mathrm{TM}}$ and Oxoid ${ }^{\circledR}$ Brilliance VRE were more selective than bioMérieux chromID ${ }^{\mathrm{TM}}$ VRE and easier to use. 


\section{C5}

EVALUATION OF THE INVERNESS COLOREX (CX) AND OXOID BRILLIANT BLUE (BB) CHROMOGENIC AGAR FOR THE DETECTION AND DIFFERENTIATION OF VANCOMYCIN (VAN) RESISTANT ENTEROCOCCUS (VRE) M DESJARDINS $^{1,2}$, K JESSAMINE $^{2}$, T LEWIS $^{2}$

${ }^{1}$ The Ottawa Hospital; ${ }^{2}$ The Ottawa Hospital Research Institute, Ottawa, ON

BACKGROUND: Screening for VRE colonization continues to rely on culture based media. Although selective and differential media has improved recovery they lack specificity and have prolonged turn around times. We compare the performance of 2 chromogenic agars for the detection and identification of VRE in clinical specimens.

METHODS: Samples submitted for VRE testing were plated to Bile Esculin Azide Agar (BEAA) with $6 \mu$ of Van and then plated to CX and $\mathrm{BB}$ agar. All plates were incubated at $35^{\circ} \mathrm{C}$ in $\mathrm{O}_{2}$ and read at 24 and 48 hours. From the BEAA, black colonies were subcultured to blood agar (BA) incubated for 24 hours and Gram stain, PYR and catalase were performed on colonies from the BA. Isolates with expected results were con-

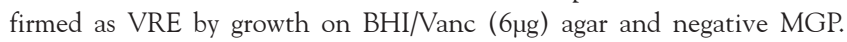
E-test was performed and the Van genotype was determined by PCR. A PYR, Van E-test and Van gene PCR was performed directly from colonies from the $\mathrm{CX}$ and $\mathrm{BB}$ agar and compared to testing performed from colonies subcultured from the chromogenic agar to BA. Sensitivity, specificity and breakthrough growth of the CX and BB plates at 24 and 48 hours were compared.

RESULTS: Of the 648 samples (605 patients) 49 were positive (39 patients) for VRE. By PFGE, $41 \%$ of the VRE had the same genotype. The $\mathrm{MIC}_{90}$ was $>256 \mathrm{mg} / \mathrm{L}$ (range 32 to $>256 \mathrm{mg} / \mathrm{L}$ ) and $90 \%$ and $10 \%$ carried the Van A and VanB genes respectively. At 24 hours, the sensitivity and specificity of both CX and BB was $67 \%$ and $99 \%$ respectively and at 48 hours, $100 \%$ and $95 \%$ respectively for the CX and $100 \%$ and $85 \%$ respectively for BB. There was 100\% correlation for direct E-test and Van PCR testing from $\mathrm{CX}$ and $\mathrm{BB}$.

CONCLUSION: Both CX and BB agar performed well and identified all VRE at 48 hours. With breakthrough of Van susceptible Enterococci on both chromogenic agars suspicious colonies will require confirmatory testing. Overall the CX agar performed better with lower rates of breakthrough compared to BB agar. Direct PYR and PCR testing of suspicious colonies from the CX will improve turn around times.

\section{C6}

EVALUATION OF THE VITEK ${ }^{\text {TM }}$ AST-GP67 CARD FOR DETECTION OF MRSA - RESULTS FROM >1300 CLINICAL STAPHYLOCOCCUS AUREUS ISOLATES

\section{P LAGACÉ-WIENS ${ }^{1,2}$, M ALFA $^{1,2}$, K MANICKAM $^{1,2}$}

${ }^{1}$ Diagnostic Services of Manitoba; ${ }^{2}$ Department of Medical Microbiology and Infectious Diseases, University of Manitoba, Winnipeg, MB

OBJECTIVE: To validate the methicillin-resistant Staphylococcus aureus screening (FOX) wells of the Vitek ${ }^{\mathrm{TM}}$ AST-GP67 card alone and the Vitek $^{\mathrm{TM}}$ Expert system against the CLSI-recommended cefoxitin disk method for detection of MRSA clinical isolates in a tertiary care hospital. METHODS: 1316 clinical S. aureus isolates were subjected to cefoxitin disk (10 mg) screening for MRSA using the CLSI recommended disk-diffusion method. Results from the disk-diffusion method were compared to the FOX MRSA screening well of the AST-GP67 card and to the Vitek ${ }^{\text {TM }}$ extert system result. Discordant results were evaluated using PBP2' latex agglutination and mecA PCR.

RESULTS: 389 (29.6\%) of the isolates were MRSA and 928 (70.4\%) were MSSA. All discordant results were resolved by PBP2' latex agglutination or mecA PCR in favour of the CLSI disk diffusion method. The FOX well alone had a sensitivity of $97.4 \%$ and a specificity of $99.4 \%$. The positive predictive value of the FOX well alone was $98.4 \%$ and the negative predictive value was $98.9 \%$. The Vitek ${ }^{\mathrm{TM}}$ expert system had a sensitivity of $98.2 \%$ and a specificity of $99.0 \%$. The positive predictive value of the Vitek ${ }^{\mathrm{TM}}$ expert system was $97.7 \%$ and the negative predictive value was $99.2 \%$.

\begin{tabular}{lrc} 
& MRSA & MSSA \\
\hline Vitek FOX+ & 378 & 6 \\
Vitek FOX- & 10 & 922 \\
Total & 388 & 928 \\
& MRSA & MSSA \\
\hline Vitek expert MRSA & 381 & 9 \\
Vitek expert MSSA & 7 & 919 \\
Total & 388 & 928 \\
\hline
\end{tabular}

CONCLUSION: Both the FOX well alone and the Vitek ${ }^{\mathrm{TM}}$ expert have a high sensitivity and specificity for detection of MRSA from clinical isolates, however the clinical and infection control consequences of false negative MRSA, which at a frequency of $1.8 \%$ for the expert system and $2.6 \%$ for the FOX well, may be serious. Individual laboratories, in collaboration with clinicians and infection control practitioners may want to consider additional MRSA screening in selected situations or in response to clinical or hospital requirements.

\section{ROOM: ST-ANTOINE B}

\section{SESSION D}

\section{D1}

\section{CHARACTERIZATION OF CARBAPENEM-RESISTANT ACINETOBACTER BAUMANII AND PSEUDOMONAS SPP. IN CANADA}

LF MATASEJE ${ }^{1}$, D BOYD ${ }^{1}$, E BRYCE ${ }^{2}$, D ROSCOE ${ }^{2}$, J EMBREE ${ }^{3}$, D GRAVEL ${ }^{4}$, K KIBSEY $^{5}$, A MOUNCHILI ${ }^{4}$, A SIMOR 6 , M KUHN ${ }^{7}$, N TURGEON ${ }^{8}$, E THOMAS $^{9}$, G TAYLOR ${ }^{10}$, M MULVEY $^{1}$

${ }^{1}$ National Microbiology Laboratory, Public Health Agency of Canada, Winnipeg, MB; ${ }^{2}$ Vancouver General Hospital, Vancouver, BC; ${ }^{3}$ University of Manitoba, Pediatric Infectious Diseases, Winnipeg, MB; ${ }^{4}$ Centre for Communicable Diseases and Infection Control, Public Health Agency of Canada, Ottawa, ON; ${ }^{5}$ Victoria General Hospital, Victoria, BC; ${ }^{6}$ Sunnybrook Health Science Centre, Toronto, ON; ${ }^{7}$ The Moncton Hospital, Moncton, NB; ${ }^{8}$ Hotel-Dieu de Quebec du CHUQ, Quebec City, QC; ${ }^{9}$ Children's and Women's Health Centre, Vancouver, $\mathrm{BC}$; ${ }^{10}$ University of Alberta, Edmonton, $\mathrm{AB}$ OBJECTIVES: Carbapenem-resistance (CARB-R) in Gram-negatives due to carbapenemases has increased globally. We describe the incidence of CARB-R Acinetobacter baumanii (AB) and Pseudomonas spp. (PS) in Canada.

METHODS: Surveillance was conducted at 13 hospital sites across Canada over one year (September 01, 2009-10). All CARB-R AB and PS isolates were submitted to the NML. Carbapenem MICs were confirmed by Etest. All isolates were screened for carbapenemases (blaNDM, blaKPC, blaIMP, blaVIM, blaGES) and $\beta$-lactamases (blaSHV, blaTEM, blaCTX, blaOXA-1, blaCMY-2) using PCR. AB were screened for blaOXA-23, -24, -58 and -51 by PCR. PS and AB were characterized by PFGE using SpeI and ApaI, respectively. Modified Hodge Test (MHT) was conducted on AB using ertapenem (ERT) and meropenem (MEM).

RESULTS: A total of $317 \mathrm{AB}(\mathrm{n}=13)$ and PS $(\mathrm{n}=304)$ isolates were sent to the NML, of which, 219 (67\%) were CARB-R by Etest; AB $(n=10)$ P aeruginosa $(P A)(n=209)$. Ten carbapenemase genes were identified in PA: blaGES-5 ( $\mathrm{n}=3$ ) and blaVIM-4 ( $\mathrm{n}=7)$. blaOXA-23 was identified in 9 of $10 \mathrm{AB}$ isolates. $\mathrm{AB}$ isolates possessed blaTEM $(\mathrm{n}=5)$, blaCTX $(\mathrm{n}=1)$ and PA, blaCTX $(\mathrm{n}=1)$. PFGE of the blaVIM PA revealed a cluster which contained 6 fingerprint patterns with $>95 \%$ similarity, all of which were identified from a single site. PFGE of AB revealed one cluster of 3 isolates from the same site. MHT using ERT was positive for all $A B$ harbouring OXA-23.

CONCLUSIONS: Hospital screening methods give higher MICs to CARBs than determined by Etest. A total of 19 carbapenemases were identified in one year of surveillance in Canada. 


\section{D2}

PREVALENCE OF BROAD SPECTRUM BETA LACTAMASE PRODUCING ENTEROBACTERIACEAE IN STOOL SAMPLES SUBMITTED TO OKANAGAN REGION HOSPITALS IN BRITISH COLUMBIA

\section{HOOPER $^{1}$, V HADWELL ${ }^{2}$, E BLONDEL-HILL ${ }^{2,3}$}

${ }^{1}$ University of Victoria, Victoria; ${ }^{2}$ Kelowna General Hospital, Kelowna; ${ }^{3}$ University of British Columbia, Vancouver, BC OBJECTIVES: Broad spectrum beta-lactamases (BSBL) including extended spectrum beta-lactamases (ESBL), AmpC cephalosporinases (AmpC) and carbapenemases(CPM) are rapidly spreading worldwide resulting in limited therapeutic options for infections due to Enterobacteriaceae. This study was designed to determine the prevalence of fully expressed BSBL in the Okanagan region.

METHODS: Routine stool and rectal samples collected from 3 hospitals in the region were screened over a 60 day period using MacConkey plates containing Cefotaxime and Imipenem discs. All isolates testing intermediate or resistant by revised CLSI guidelines were identified and subjected to phenotypic tests to exclude ESBL, AmpC and CPM.

RESULTS: Eighty-four of 759 stool samples screened required further testing to rule out BSBLs. A BSBL was found in 76 stool samples. Fully expressed AmpC producing organisms were found in $6.6 \%$ of stool samples while ESBLs were found in $2.4 \%$. Three samples had E coli with both AmpC and ESBL. No $\mathrm{CPM}$ was found although $2 \mathrm{AmpC}$ positive $E$ coli isolates had decreased susceptibility to ertapenem presumably due to permeability mutations.

CONCLUSIONS: Ten percent of stool samples submitted to 3 laboratories in the Okanagan region over a two month period contained Enterobacteriaceae with BSBLs. Fully expressed AmpC (chromosomal or plasmid) are almost three times as common as ESBLs. No transmissible carbapenemases were detected although decreased susceptibility to ertapenem was noted in AmpC producing E coli $(<0.5 \%)$.

\section{D3}

\section{HOUSEHOLD TRANSMISSION OF COMMUNITY- AND HEALTHCARE-ASSOCIATED METHICILLIN RESISTANT STAPHYLOCOCCUS AUREUS STRAINS}

K KATZ $^{1,2}$, P GNANASUNTHARAM ${ }^{3}$, A FAHEEM ${ }^{1}$, A GELOSIA ${ }^{3}$, L LOUIE ${ }^{4}$, AJ MCGEER ${ }^{2,3}$, M MULLER $^{2,5}$, W NG $^{1}$, K OSTROWSKA ${ }^{6}$, D RICHARDSON ${ }^{7}$, L VERNICH $^{2}$, H WONG $^{4}$, A SIMOR $^{2,4}$

${ }^{1}$ North York General Hospital; ${ }^{2}$ University of Toronto; ${ }^{3}$ Mount Sinai Hospital; ${ }^{4}$ Sunnybrook Health Sciences Centre; ${ }^{5}$ St Michael's Hospital; ${ }^{6}$ Trillium Health Centre, Toronto; ${ }^{7}$ William Osler Health System, Brampton, ON

OBJECTIVE: To describe the transmission of community and healthcare MRSA strains among close contacts (CC) in household (HH) settings. METHODS: Index cases (IC) with MRSA colonization/infection were identified among patients presenting to participating institutions. Consenting $\mathrm{HH}$ with an IC and a minimum of one close contact (CC), spending more than 20 hrs in the $\mathrm{HH}$, were eligible for inclusion. Baseline questionnaires were completed and surveillance cultures collected from multiple body sites. Three monthly follow-up visits were undertaken for families with a minimum of one non-colonized CC. MRSA surveillance cultures were performed using standard clinical laboratory methods. The first MRSA isolate from each IC/CC underwent PFGE, SCCmec typing and PVL testing by standard methods. Strains carrying SCCmec IV/V were considered CA-MRSA, and those carrying SCCmec II were considered HA-MRSA.

RESULTS: 27 IC (11 CA-MRSA, 11 HA-MRSA and 5 untyped) and $59 \mathrm{CC}$ are described. In $6(22 \%) \mathrm{HH}$, all CC were colonized at the baseline visit; 6/6 (100\%) HA-MRSA. Overall, 14/22 (63\%) and 14/31 (45.2\%) of typed CC of IC with HA-MRSA and CA-MRSA strains, respectively, were colonized at some point over the 4 months $(\mathrm{p}=0.18) .64 \%$ of the $28 \mathrm{CC}$ colonizations were first detected at baseline, $17.9 \%$ on first, $14.3 \%$ on second, and $3.6 \%$ on $3 \mathrm{rd}$ f/u visit. All IC/CC strains matched within $\mathrm{HH}$. Recruitment is ongoing.

CONCLUSION: MRSA transmission of both CA- and HA-MRSA strains among CC of MRSA-colonized IC is common. Delineation of transmission risk factors in $\mathrm{HH}$ settings is required.

\section{D4}

\section{GASTRO-INTESTINAL OUTBREAKS IN LONG TERM CARE FACILITIES, HOSPITAL AND DAY CARE CENTRES IN BRITISH COLUMBIA}

N PRYSTAJECKY ${ }^{1,2}$, B AUK ${ }^{1}$, J WONG ${ }^{1}$, J FUNG ${ }^{1}$, B WONG ${ }^{1}$, P TANG ${ }^{1,2}$, J ISAAC-RENTON ${ }^{1,2}$

${ }^{1}$ British Columbia Centre for Disease Control Public Health and Reference Laboratory (BCPHL); ${ }^{2}$ University of British Columbia Department of Pathology and Laboratory Medicine, Vancouver, BC INTRODUCTION: Gastro-intestinal (GI) viruses are a leading cause of illness in children, elderly and the immunocompromised. These viruses, which include norovirus, rotavirus and adenovirus, are easily aerosolized and spread person-to-person, leading to large outbreaks in community settings such as hospitals, long-term care facilities (LTCF) and day care centres. This work was performed to evaluate GI outbreak patterns in different facility types.

METHODS: GI outbreaks were reported by local health authorities, infection control practitioners or local facilities managers. Testing for this study period was carried out in the BC Public Health \& Microbiology Reference Laboratories (BC PHL). Upon receipt, samples were first screened for norovirus by RT-PCR. Further testing for rotavirus, adenovirus, bacterial and/or parasitic pathogens were carried out as according to British Columbia (BC) outbreak investigation protocols. A retrospective analysis from 2006 until 2010 was carried out using BC PHL data.

RESULTS: GI outbreaks in BC peaked in 2006-07 with some seasonal variation noted. A year over year decrease in numbers of GI outbreaks was then noted. Outbreaks in different facility types, however, exhibited distinctly different patterns; in hospital settings, outbreaks peaked in 2006-07 followed this steady decrease in numbers of outbreaks per year. Outbreak patterns for LTCFs, on the other hand, exhibited a cyclical pattern while lacking a significant peak year as compared to the hospitals. In contrast, outbreaks in day care centres showed a similar pattern as hospital but with peak numbers of outbreak in 2007-08 season instead. These patterns hold when evaluating all GI outbreaks or for norovirus outbreaks alone (most common etiological agent in outbreaks).

DISCUSSION: Annual GI outbreak patterns differed amongst facility types. Recent work in Alberta indicated a cyclical pattern of norovirus transmission, likely reflecting genetic drift. In BC, this cyclical trend was observed only in LTCFs. This likely reflects differences in GI virus transmission in different facility types. Knowledge of transmission dynamics amongst facility type may facilitate infection control activities and ameliorate public health vigilance.

\section{D5}

\section{A SHELTER ASSOCIATED TUBERCULOSIS OUTBREAK: LESSONS FORGOTTEN}

D MOREAU ${ }^{1}$, R AHMED ${ }^{1,2}$, E DER ${ }^{1}$, D KUNIMOTO ${ }^{1,2}$, L CHUI $^{3,4}$

${ }^{1}$ Edmonton TB Clinic; ${ }^{2}$ Department of Medicine, University of Alberta; ${ }^{3}$ Provincial Laboratory for Public Health; ${ }^{4}$ Laboratory Medicine and Pathology, University of Alberta, Edmonton, AB OBJECTIVE: To describe the dynamics of a multiethnic, multicenter inner city tuberculosis (TB) outbreak and the consequence of delayed diagnosis.

METHODS: Clinical, public health, and molecular epidemiologic data were used to identify and describe a complex cluster of TB cases occurring within an urban inner city postal code area.

RESULTS: Between May 2008 to May 2009, 3 cases of active TB were linked by $\mathrm{M}$ tuberculosis genotype and residence of each case within an urban apartment building. An additional cluster of 16 cases of TB disease were identified in June to December 2009 and linked to 2 homeless shelter facilities. The majority of these cases were linked epidemiologically and through genotype to the initial urban apartment building cluster. All cases, except one, occurred in Canadian-born Aboriginal (30\%) or North East African (65\%) groups. These two groups were not otherwise socially linked to one another. $80 \%$ of cases had pulmonary TB and $>50 \%$ had smear positive, cavitary disease. The emergency department was the initial site of presentation for $>50 \%$ of cases, averaging $>2$ visits prior to diagnosis. 
Overall, 925 contacts were identified of which 655 contacts were unable to be located.

CONCLUSIONS: This study illustrates the need for improved strategies for early diagnosis of active TB. It also highlights the need for a collaborative approach between inner city agencies, public health employees, and health care providers involved in the care of homeless populations in order to reduce the transmission of TB and aid in contact investigation.

\section{D6}

\section{A MATHEMATICAL MODEL OF NOSOCOMIAL CLOSTRIDIUM DIFFICILE INFECTION (CDI) TRANSMISSION IN AN ACUTE CARE HOSPITAL SYSTEM WITH SEASONAL VARIATIONS IN TRANSMISSION RATE}

\section{K BROWN, D FISMAN}

Dalla Lana School of Public Health, Toronto, ON

OBJECTIVE: Clostridium difficile, a toxin-producing bacterium that causes diarrhoea, is the largest single cause of morbidity and mortality among hospital-acquired infections, and with over 5,000 cases occurring annually in Ontario. Wintertime increases in CDI incidence are known and some authors have noticed a correlation between the incidence of CDI and influenza. Our aim was to develop a mathematical model of CDI incidence and to explore whether seasonal forcing due to wintertime increases in transmission rate could explain seasonal increases in CDI incidence.

METHODS: A simple mathematical model was developed to predict CDI incidence as a function of the transmission rate per day, the factor of increase in the transmission rate during the flu season, as well as the mean date and duration of the flu season. The model was calibrated to CDI incidence in all Quebec acute care centres during the pre-epidemic period from April $1^{\text {st }}$ 1998, to March 31 st, 2002 , derived from the Quebec Discharge Abstract Database (MED-ÉCHO) and FluWatch data for Quebec.

RESULTS: The mean incidence in the 46 -month periods beginning October $1^{\text {st }}$ was 6.98 cases per 1,000 admissions versus and 5.46 per 1,000 admissions in the 4 periods beginning April $1^{\text {st }}$. In the base-case analysis, the model ascertained a transmission rate of 0.026 cases per person-day of exposure which increased seasonally to $0.028(+9.7 \%)$ in the 3 month period starting October $1^{\text {st }}$. When the increase in CDI transmission rate was pegged to the flu season using FluWatch data, error in the prediction of the date of peak annual CDI incidence was reduced by $28.2 \%$. CONCLUSION: Our mathematical model confirms that small seasonal increases in the CDI transmission rate amplify wintertime CDI incidence, and may explain temporal patterns of incidence in North American hospitals.

FRIDAY, APRIL 8
ROOM: VILLE-MARIE A

\section{SESSION E}

\section{E1}

\footnotetext{
ANTIMICROBIAL RESISTANCE IN PATHOGENS ISOLATED FROM CANADIAN HOSPITAL CLINICS, EMERGENCY ROOMS, MEDICAL/SURGICAL WARDS AND INTENSIVE CARE UNITS: RESULTS OF THE CANWARD 2010 STUDY M DeCORBY $^{1}$, HJ ADAM ${ }^{1,2}$, LF MATASEJE ${ }^{3}$, M MULVEY ${ }^{1,3}$, JA KARLOWSKY ${ }^{1,2}$, P LAGACE-WIENS ${ }^{1,2}$, A WALKTY1,2, DJ HOBAN ${ }^{1}, 2$, GG ZHANEL ${ }^{1}$

${ }^{1}$ University of Manitoba; ${ }^{2}$ Diagnostic Services of Manitoba;

${ }^{3}$ National Microbiology Laboratory, Winnipeg, MB

OBJECTIVES: The CANWARD study assesses the pathogens causing infections in patients affiliated with Canadian hospitals and evaluates the prevalence of antimicrobial resistance in these isolates.

METHODS: 14 centres across Canada submitted pathogens causing infections from patients attending clinics $(\mathrm{C})$, emergency rooms (ER), medical and surgical wards (W) and intensive care units (ICU) in 2010. Susceptibility testing was performed by CLSI microdilution methods.
}

RESULTS: 4,555 isolates were collected: $44.9 \%, 26.4 \%, 15.2 \%$, and $13.5 \%$ from blood, respiratory, urine and wound/IV site specimens, respectively. Isolates were from patients on W $36.6 \%$, ER 25.6\%, C 20.0\%, and ICU $17.8 \%$. The most common pathogens were: E coli $21.6 \%, S$ aureus (MSSA) $17.3 \%$, P aeruginosa $8.0 \%$, K pneumoniae 6.2\%, and $E$ faecalis $4.9 \%$. The overall prevalence of MRSA, VRE, and ESBL- E coli was: $21.1 \%, 4.0 \%$, and $2.5 \%$, respectively. Resistance rates (RR) for $E$ coli were: $0 \%$ meropenem (MER), $0 \%$ tigecycline (TGC), 1.7\% piperacillin/tazobactam (PTZ), 5.4\% ceftriaxone (CTR), 7.9\% gentamicin (GEN), 21.6\% ciprofloxacin (CIP) and $25.7 \%$ trimethoprim/sulfamethoxazole (SXT). RR for $P$ aeruginosa were: $0.9 \%$ colistin (COL), 6.0\% PTZ, 8.1\% MER, $16.3 \%$ GEN and $18.9 \%$ CIP. RR for MRSA were: $0 \%$ vancomycin (VAN), linezolid (LZD), and daptomycin (DAP), 5.6\% SXT, 44.3\% clindamycin, $81.9 \%$ CIP, and $86.9 \%$ clarithromycin.

CONCLUSIONS: RR for E coli were lowest with MER, TGC and PTZ, while RR for $P$ aeruginosa were lowest with COL, PTZ, and MER. For MRSA, no resistance occurred with VAN, LZD, or DAP.

\section{E2}

VANCOMYCIN-RESISTANT ENTEROCOCCI (VRE) FINDINGS FROM THE CANADIAN NOSOCOMIAL INFECTION SURVEILLANCE PROGRAM (CNISP), 2006-2009 $\underline{\text { R MITCHELL }}^{1}$, J EMBIL ${ }^{2}$, J CONLY ${ }^{3}$, D GRAVEL ${ }^{1}$, L JOHNSTON ${ }^{4}$, S LEDUC $^{1}$, A MATLOW ${ }^{5}$, M MULVEY ${ }^{1,6}$, D ORMISTON ${ }^{2}$, A SIMOR ${ }^{7}$, A WONG ${ }^{8}$, D ZOUTMAN ${ }^{9}$; Canadian Nosocomial Infection Surveillance Program ${ }^{1}$

${ }^{1}$ Centre for Communicable Diseases and Infection Control, Public Health Agency of Canada, Ottawa, ON; ${ }^{2}$ Health Sciences Centre, Winnipeg, MB; ${ }^{3}$ Foothills Medical Centre, Calgary, $\mathrm{AB} ;{ }^{4} \mathrm{Queen}$ Elizabeth II Health Sciences Centre, Halifax, NS; ${ }^{5}$ The Hospital for Sick Children, Toronto, ON; ${ }^{6}$ National Microbiology Laboratory, Winnipeg, MB; ${ }^{7}$ Sunnybrook Health Sciences Centre, Toronto, ON; ${ }^{8}$ Royal University Hospital, Saskatoon, SK; ${ }^{9}$ Kingston General Hospital, Kingston, ON

OBJECTIVE: To describe the epidemiology of VRE in participating CNISP hospitals between 2006 and 2009.

METHODS: Laboratory surveillance for VRE was conducted through the collection of screening specimens and clinical isolates from inpatients in CNISP hospitals. Clinical isolates were sent to the National Microbiology Laboratory for molecular testing.

RESULTS: Forty-one hospitals in eight provinces identified 9,502 VRE infected and colonized cases. Most cases $(94 \% ; 8,934)$ acquired VRE in hospital; $65 \%(6,158)$ were 65 years or older; and $64 \%(6,115)$ were from central Canada (Ontario and Quebec). From 2006 to 2009, the overall rate of VRE significantly increased from 1.19 to 4.98 cases per 1,000 patient admissions $(p<0.001)$; the rate of VRE colonization increased from 1.13 to 4.74 per 1,000 patient admissions $(p<0.001)$ and the rate of VRE infection increased from 0.06 to 0.24 infections per 1,000 patient admissions $(p<0.001)$. Four per cent $(381)$ of all VRE cases were infections; $41 \%$ were urinary tract infections, $38 \%$ post surgical soft tissue infections, $20 \%$ bloodstream infections and 3\% catheter-associated bloodstream infections. Of all VRE cases, $5 \%$ (502) had a positive $\mathrm{C}$ difficile laboratory test prior to VRE positive culture while $6 \%(n=552)$ had a concurrent MRSA infection. The majority of the 232 clinical isolates sent for molecular testing were E. faecium vanA $(87 \% ; 202)$.

CONCLUSIONS: There has been an increase in both VRE infection and colonization rates in CNISP hospitals since 2006. The increase in VRE has important implications as there are limited treatment options available. 


\section{E3}

\section{CHARACTERIZATION OF CARBAPENEM-RESISTANT ENTEROBACTERIACEAE IN CANADA: RESULTS OF THE CANADIAN NOSOCOMIAL SURVEILLANCE PROGRAM (CNISP)}

LF MATASEIE ${ }^{1}$, D BOYD ${ }^{1}$, E BRYCE ${ }^{2}$, D ROSCOE ${ }^{2}$, J EMBREE ${ }^{3}$, D GRAVEL ${ }^{4}$, P KIBSEY ${ }^{5}$, A MOUNCHILI ${ }^{4}$, A SIMOR $^{6}$, M KUHN $^{7}$, N TURGEON ${ }^{8}$, E THOMAS ${ }^{9}$, G TAYLOR ${ }^{10}$, M MULVEY $^{1}$

${ }^{1}$ National Microbiology Laboratory, Public Health Agency of Canada, Winnipeg, MB; ${ }^{2}$ Vancouver General Hospital, Vancouver, BC; ${ }^{3}$ University of Manitoba, Pediatric Infectious Diseases, Winnipeg, MB; ${ }^{4}$ Centre for Communicable Diseases and Infection Control, Public Health Agency of Canada, Ottawa, ON; ${ }^{5}$ Victoria General Hospital, Victoria, BC; ${ }^{6}$ Sunnybrook Health Science Centre, Toronto, ON; ${ }^{7}$ The Moncton Hospital, Moncton, NB; ${ }^{8} \mathrm{Hotel}-\mathrm{Dieu}$ do Quebec du CHUQ, Quebec City, QC; ${ }^{9}$ Children's and Women's Health Centre, Vancouver, BC; ${ }^{10}$ University of Alberta Hospital, Edmonton, $\mathrm{AB}$

OBJECTIVES: Rapid emergence of carbapenem-resistant (CARB-R) Enterobacteriaceae (ENT) globally prompted us to investigate the occurrence of CARB-R ENT in Canada.

METHODS: Surveillance was conducted at 13 hospital sites across Canada over a one year period (September 01, 2009-10). ENT with a carbapenem MIC of $\geq 2 \mathrm{mg} / \mathrm{L}$ were submitted to the NML. CARB MICs were confirmed by Etest. All isolates were screened for carbapenemases (blaNDM, blaKPC, blaIMP, blaVIM, blaGES) and $\beta$-lactamases (blaSHV, blaTEM, blaCTX, blaOXA-1, blaCMY-2) using PCR. Modified Hodge Test (MHT) was done using ertapenem (ERT) and meropenem (MEM).

RESULTS: A total of 137 ENT were sent to the NML, of which, 62 (45.3\%) were $\geq 2 \mathrm{mg} / \mathrm{L}$ by Etest. 10 carbapenemase genes were identified: blaKPC-3 $(\mathrm{n}=6$; Klebsiella pneumoniae (KP), K.oxytoca, and E.coli (EC)), blaNDM-1 $(\mathrm{n}=2 ; \mathrm{KP}, \mathrm{EC})$ blaVIM-2 ( $\mathrm{n}=1$; Enterobacter spp.(EB)) and blaSME $(\mathrm{n}=1$; Serratia spp.(SER)). Additionally, blaKPC and blaNDM isolates also harboured blaSHV $(\mathrm{n}=4, \mathrm{n}=1)$ blaTEM $(\mathrm{n}=6, \mathrm{n}=1)$ blaCTX $(\mathrm{n}=2, \mathrm{n}=2)$ blaOXA-1 $(\mathrm{n}=0, \mathrm{n}=2)$ and blaCMY-2 $(\mathrm{n}=0, \mathrm{n}=2)$ respectively. MHT was positive (POS) for all organisms harbouring known carbapenemases. $17 \mathrm{~EB}$ (BL negative) were MHT-POS. 1 Proteus spp. (CMY-2), 1 KP (SHV,CTX) and 1 SER were MHT-POS. The SER was phenotypically metallo$\beta$-lactamase (MBL) POS.

CONCLUSIONS: Hospital screening methods give higher MICs to CARBs than determined by Etest. 10 carbapenemases were identified in Canada over one year. MHT was sensitive but susceptible to false POS especially in EB.

\section{E4}

\section{CANADA'S FIRST CASE OF A MULTI-DRUG RESISTANT CORYNEBACTERIUM DIPHTHERIAE, ISOLATED FROM A SKIN ABSCESS}

N MINA ${ }^{1}$, K BERNARD $^{2,3}$, T BURDZ ${ }^{2}$, D WIEBE ${ }^{2}$, JS RAI ${ }^{4}$, L HOANG ${ }^{5,6}$

${ }^{1}$ University of Alberta, Department of Medical Microbiology, Edmonton, AB; ${ }^{2}$ National Microbiology Laboratory, Public Health Agency of Canada; ${ }^{3}$ University of Manitoba, Department of Medical Microbiology, Winnipeg, MB; ${ }^{4}$ Family Physician, Delta; ${ }^{5}$ BCCDC Public Health Reference Microbiology Laboratory, Provincial Health Services Authority; ${ }^{6}$ University of British Columbia, Department of Pathology and Laboratory Medicine, Vancouver, BC

OBJECTIVE: We report on a case of a toe wound infection caused by a multi-drug resistant (MDR) Corynebacterium diphtheriae.

METHOD: Retrospective case review was performed. The bacterium was recovered and identified using standard methods at the local and provincial laboratories, then forwarded to the National Microbiology Laboratory for toxin analysis. Antimicrobial susceptibility testing and typing (by multilocus sequence typing [MLST]) were done; ermX was detected by PCR. RESULTS: The patient was a non-immunized 38y old male with a recent travel history to India, who presented with a skin abscess. The isolate was identified as C diphtheriae biotype mitis and toxigenic. MLST type was ST
136, a type unique to the NML database and published literature. The isolate was resistant to clindamycin and erythromycin (ermX gene positive) as well as to tetracycline and trimethoprim/sulfamethoxazole with intermediate values towards ceftriaxone and cefotaxime. Although no interpretation guidelines exists for telithromycin and chloramphenicol, the isolate demonstrated high MICs (micrograms $/ \mathrm{ml}$ ) of $>4$ and 32 respectively. A variety of drug classes appeared to be sensitive including penicillin, meropenem, cefepime, vancomycin, daptomycin, gentamicin, and linezolid.

CONCLUSION: Our study demonstrated the 1st reported Canadian case involving a MDR strain of $\mathrm{C}$ diphtheriae causing wound infection. There has been no evidence of spread of this strain. Resistance to 4 drug classes among $\mathrm{C}$ diphtheriae have only very rarely been described in recent global literature.

\section{E5}

\section{DAPTOMYCIN FOR HIGH GRADE GRAM POSITIVE INFECTIONS: A CANADIAN TERTIARY HOSPITAL EXPERIENCE}

\section{S HAIDER, A BROOKS}

McMaster University, Hamilton Health Sciences, Hamilton, ON OBJECTIVES: Vancomycin has been the treatment of choice for MRSA high grade infections at most Canadian Centers, but growing concern about treatment failures, and concerns of population creep in vancomycin MIC has focused the need for newer treatment options. Daptomycin has been studied for treatment of infective endocarditis and or MRSA bacteremia, and soft tissue infections. Daptomycin was approved for treatment of MRSA endocarditis and/or MRSA bacteremia, or intolerance to vancomycin for documented gram positive infections at our center.

METHODS: A retrospective chart review using Pharmacy Records and Electronic Medical Records was done for the period 2008-2010. Baseline epidemiological data on patients will be reported along with clinical and microbiological response rates. Safety and tolerability data on daptomycin use will be reported.

RESULTS: Baseline characteristics: 33 patients: age range (19-82 yrs). $30 \%(11 / 33)$ of patients had MRSA infections, while VRE infections was the second most common indication for use of daptomycin seen in $(8 / 33)$ patients. Clinical conditions included: endocarditis $8 / 33$, bacteremia $8 / 33$, and complicated skin/soft tissue infections or bone/joint infections in 15/33 patients. Daptomycin was well tolerated. There was no microbiological failures on daptomycin.

CONCLUSIONS: Our single center experience highlights the need for newer treatment options such as daptomycin for hospitalized patients with MRSA, VRE, and other high grade gram positive infections.

\section{E6}

SYMPTOM-REPORTING COMPARED WITH AUDIOMETRY FOR THE DETECTION OF OTOTOXICITY IN PATIENTS ON LONG-TERM AMINOGLYCOSIDE THERAPY

\section{PALMAY $^{1}$, SAN WALKER ${ }^{1,2}$, SE WALKER ${ }^{1,2}$, A SIMOR $^{1,2}$}

${ }^{1}$ Sunnybrook Health Sciences Centre; ${ }^{2}$ University of Toronto, Toronto, ON

OBJECTIVE: Auditory toxicity (cochleotoxicity) is a concern in patients receiving prolonged aminoglycoside therapy. Published data comparing symptom monitoring to audiometry testing for the detection of aminoglycosideinduced cochleotoxicity is absent, and agreement regarding the optimal monitoring of these patients for early detection of this detrimental effect is lacking. The objective of this study was to compare the sensitivity of symptom monitoring to audiometry in identifying auditory toxicity in patients on prolonged aminoglycoside therapy.

METHODS: A retrospective chart review of adult hospitalized patients prescribed prolonged aminoglycoside therapy ( $\geq 21$ days) who completed at least one audiometry test between January $1^{\text {st }} 1999$ to December $31^{\text {st }} 2009$, was conducted. Data pertaining to results of audiometry testing and development of symptoms of auditory toxicity was collected. Audiometry was compared with symptom monitoring for the detection of auditory toxicity. RESULTS: A total of 40 eligible patients were included for analysis. Audiometry was significantly better than symptom monitoring to identify 
early ototoxicity $(p=0.023 ; A R R=17.5 \%$ and $N N T=6)$. The sensitivity, negative predictive value and accuracy in detecting early cochleotoxicity with symptom monitoring is only $61 \%, 75 \%$ and $82 \%$, respectively.

CONCLUSION: Audiometry testing is significantly better than monitoring symptoms for aminoglycoside-induced auditory toxicity in patients on prolonged aminoglycoside therapy, and is recommended as routine monitoring for patients at risk of aminoglycoside-induced cochleotoxicity.

\section{ROOM: ST-ANTOINE A}

\section{SESSION F}

\section{F1}

\section{SELECTION OF DNA APTAMERS SPECIFIC FOR GROUP A STREPTOCOCCAL M TYPES \\ C HAMULA, X LI, C LE}

University of Alberta, Edmonton, AB

OBJECTIVE: Epidemiological surveillance of Group A Streptococcal (GAS) clinical isolates is important for outbreak management as well as vaccine development and implementation. Due to the complexity of existing methods, typing of GAS isolates has tended to be centralized in laboratories specializing in GAS characterization. Alternative GAS typing methods are needed to extend the range of GAS typing outside of reference laboratories. The protein-based serotyping system of GAS makes it an ideal aptamer target. Aptamers are short, synthetic, single-stranded nucleic acids that bind a target molecule strongly and specifically. DNA aptamers possess key advantages over antibodies including ease of production, longer shelf-life, low variability between batches, and ease of chemical modification. The objective of this study is to create aptamers that bind with high specificity and affinity to live GAS cells, and to isolate those aptamer sequences that bind to the GAS M protein and are specific for different GAS M types.

METHODS: To eliminate the need of purifying target molecules from the cell surface, we have developed a technique using whole live bacterial cells in suspension as targets, to select for single-stranded DNA aptamers specific to bacterial surface molecules. We are currently applying this technique to a mixture of the 10 most prevalent invasive GAS M-types in Canada: M1, M2, M3, M4, M6, M11, M12, M28, M77 and M89.

RESULTS: Aptamer pools obtained after 8-20 rounds of selection demonstrate increased affinity for and selective binding with a mixture of GAS cells. A total of 102 aptamers from 5 different pools were cloned, sequenced, and subjected to further characterization. Several sequences have been isolated with nanomolar-level binding affinity and high species specificity. M-type specific aptamers have also been discovered.

CONCLUSION: Aptamers that bind to bacterial cell surface molecules could prove useful in bacterial surveillance and typing, diagnosis of bacterial infections, and therapeutics. Aptamers offer myriad advantages over antibodies. Specifically, aptamers that are able to positively identify the presence of GAS and different GAS M-types will make surveillance of iGAS isolates routine both in the laboratory and potentially the clinic.

\section{F2}

\section{COST-EFFECTIVE MODIFICATION OF THE BD GENEOHM ${ }^{\mathrm{TM}}$ C DIFFICILE ASSAY}

\section{LOUIE $^{1}$, A SIMOR ${ }^{1,2}$}

${ }^{1}$ Sunnybrook Health Sciences Centre; ${ }^{2}$ University of Toronto, Toronto, ON

OBJECTIVES: The expense of molecular based assays may prevent laboratories from utilizing commercially available real-time PCR methods for the detection of $\mathrm{C}$ difficile from fecal samples. We compared frozen PCR master mix (MM) from the BD GeneOhm ${ }^{\text {TM }}$ Cdiff assay with freshly prepared MM to determine performance.

METHODS: BD GeneOhm ${ }^{\text {TM }}$ Cdiff assay master mix (MM) was reconstituted according to the manufacturer's instructions, and unused MM was aliquoted into SmartCycler tubes and frozen at $-20^{\circ} \mathrm{C}\left(\mathrm{fz}_{\mathrm{z}} \mathrm{MM}\right)$, protected from light. Aliquots were frozen between 1-7 days prior to testing. Kit controls and fresh fecal lysates were tested with the fzMM and freshMM and analyzed on 24 different runs. Performance was assessed by cycle threshold values.

RESULTS: A total of 15 positive and 19 negative specimens were tested, with 24 positive and negative kit controls. The average cycle threshold $\left(\mathrm{C}_{T}\right)$ of $\mathrm{f}_{z} \mathrm{MM}$ was determined with the positive kit control and compared with freshMM. The average $C_{T}$ value of the positive control with freshMM was 32.1 while the average $C_{T}$ value with $\mathrm{f}_{z} \mathrm{MM}$ as $31.2(P=0.10)$. FzMM testing of clinical specimens gave concordant results when compared with freshMM. No discrepancies were found and all runs were valid.

CONCLUSION: Use of $\mathrm{f}_{z} \mathrm{MM}$ worked well and did not compromise the performance of the Cdiff assay. This modification causes no reagent waste and reduces cost per test, and may allow more laboratories to use real-time PCR assays such as the BD GeneOhm ${ }^{\mathrm{TM}}$ Cdiff assay for more rapid and accurate detection of toxigenic $\mathrm{C}$ difficile.

\section{F3}

\section{DETECTION OF ENTERIC PATHOGENS IN COMMUNITY AND HOSPITAL DIARRHEA CASES USING A MULTIPLEX PCR ASSAY}

$\underline{L C H U I}^{1,2}{ }^{,}$J FUNG $^{1}$, M-C LEE $^{3}$, K MALEJCZYK $^{2}$

${ }^{1}$ Provincial Laboratory for Public Health; ${ }^{2}$ Laboratory Medicine and Pathology, University of Alberta; ${ }^{3}$ DynaLIFE DX Laboratory Services, Edmonton, $\mathrm{AB}$

OBJECTIVES: Numerous viruses and bacteria can cause diarrhea and lead to various complications. Infectious diarrhea is the leading cause of death among children worldwide. Currently, most frontline diagnostic laboratories only focus on bacterial cultures of stool samples. This study evaluates Seegene's Seeplex ${ }^{\circledR}$ Diarrhea ACE Detection assay on stool samples. The assay uses Dual Priming Oligonucleotide Multiplex PCR to simultaneously detect viruses, bacteria and bacterial toxin genes.

METHODS: Stool samples were collected from the community and hospitals during the summer of 2010. Nucleic acid was extracted and used as template for reverse transcription of the RNA viruses. PCR amplification of the cDNA or DNA was subsequently performed as per manufacturer's instructions. Amplicons were analyzed using LAB901 ScreenTape ${ }^{\circledR}$ Auto Capillary Electrophoresis or agarose gel electrophoresis. The viral panel amplified enteric adenovirus, rotavirus, norovirus, and astrovirus gene targets. The 2 bacterial panels amplified Salmonella, Shigella, Vibrio, Campylobacter, Y enterocolitica, Aeromonas, C difficile toxin B, C perfringens toxin, and Verocytotoxin-producing E coli gene targets.

RESULTS: A total of 1018 stool samples were included in the study. Norovirus GII and rotavirus were the most common viral infections, found in $3.8 \%$ and $1.6 \%$ of the cases respectively. C perfringens toxin and $\mathrm{C}$ difficile toxin B genes were identified in $9.1 \%$ and $5.0 \%$ of the cases respectively. Campylobacter was the predominant bacterial infection at $2.5 \%$.

CONCLUSIONS: Seegene's assay is time and cost-effective for diagnostic purposes and allows for the detection of a wide panel of viral and bacterial agents commonly found in diarrheal patients. Results of the assay can be reported in around 9 hours. Once a pathogen has been identified by PCR, selective plates for the specific bacteria can be cultured, thereby eliminating the unnecessary plating of a panel of pathogens. This assay can be easily implemented in a routine diagnostic molecular microbiology laboratory.

\section{F4}

CONFIRMATION OF THE ROCHE COBAS AMPLICOR 480 NEISSERIA GONORRHEA MOLECULAR ASSAY WITH AN IN-HOUSE REAL-TIME POLYMERASE CHAIN REACTION ASSAY

$\underline{\text { S DREWS }}^{1,2}$, L CHUI ${ }^{1,3,4}$, L DOUGLAS $^{1,4}$, G ZAHARIADIS ${ }^{1,3,4}$, G TYRRELL ${ }^{1,3,4}$

${ }^{1}$ ProvLab Alberta; ${ }^{2}$ Microbiology and Immunology, University of Calgary, Calgary; ${ }^{3}$ Laboratory Medicine and Pathology, University of Alberta; ${ }^{4}$ University of Alberta Hospital, Edmonton, $A B$

OBJECTIVE: To calculate the false-positive rate for the Roche COBAS AMPLICOR 480 Neisseria gonorrhea (GC) molecular assay from genital swab and urine specimens.

METHODS: Genital swab (endocervical and urethral swab specimens) 
and urine specimens from STD/family planning clinics collected in Calgary and Edmonton (01 January 2009- 20 November 2010) were analyzed. These specimens were tested for GC using the Roche COBAS AMPLICOR 480 Assay and positive samples underwent supplemental testing using an in-house real-time polymerase chain reaction (PCR) assay (Chui et al, 2008).

RESULTS: During the study period, 32373 specimens were analyzed for GC by Alberta ProvLab. 902 (2.8\%) were positive for GC using the Roche COBAS AMPLICOR 480 Assay and upon supplemental testing using an in-house real-time PCR assay, 236/902 (26.2\%) confirmed as GC. Thus the percentage of GC $16 \mathrm{~S}$ positive specimens out of all specimens received for GC screening was $(236 / 32373,0.7 \%)$.

\begin{tabular}{lcc} 
Category & Number & $\begin{array}{c}\% \text { of total } \\
\text { received }\end{array}$ \\
\hline Specimens sent for GC & 32373 & 100 \\
Specimens positive by Roch Amplicor & 902 & 2.8 \\
Specimens positive by in-house PCR & 236 & 0.7 \\
False-positive by Roche Amplicor & 666 & 2.1 \\
\hline
\end{tabular}

CONCLUSIONS: The Roche COBAS AMPLICOR 480 Assay has a significant false-positive rate in our jurisdiction. This study indicates the importance of using confirmatory assays for positive GC specimens tested by the Roche COBAS AMPLICOR 480 Assay.

Reference: Chui L, Chiu T, Kakulphimp J, Tyrrell GJ. Clin Microbiol Infect 2008 May;14(5):473-9.

\section{F5}

TAQ PROBING: A NEW MOLECULAR DIAGNOSTIC METHOD APPLIED TO DETECT SNP ASSOCIATED WITH ANTIBIOTIC RESISTANCE IN CAMPYLOBACTER JEJUNI P DEXTRAS PAQUETTE ${ }^{1}, S$ DESLANDES ${ }^{1}$, EH FROST ${ }^{1,2}$

${ }^{1}$ Université de Sherbrooke; ${ }^{2}$ Centre hospitalier universitaire de Sherbrooke, Sherbrooke, QC

OBJECTIVES: Genomic research has led to the discovery of single nucleotide polymorphisms (SNP) associated with antibiotic resistance. SNP in $\mathrm{C}$ jejuni 23S rRNA and gyrA genes, are the most common causes of resistance to erythromycin and ciprofloxacin. These antibiotics are employed to treat $\mathrm{C}$ jejuni infections, the world's most common cause of bacterial diarrhea. While many methods to detect $C$ jejuni resistance can be performed by reference lab, hospital lab will depend on quick and easy assays. METHODS: Our newly devised strategy, Taq Probing, provides a rapid, efficient and cost-effective method of detecting SNP. According to Taq Man PCR chemistry, probes are hydrolyzed to provide a signal. We developed a strategy to preserve some probes from hydrolysis and use them to form a post-PCR probe-amplicon complex. The melting temperature (Tm) of this complex depends on the presence (or absence) of resistance SNP. RESULTS: We analysed $73 \mathrm{C}$ jejuni colonies known to be resistant to either or both of erythromycin or ciprofloxacin. Using a protocol that detected both mutations simultaneously, we detected the resistance SNP in 23 of 27 erythromycin resistant and 35 of 46 ciprofloxacin resistant colonies. CONCLUSIONS: Alternative explanations for resistance in the other bacteria are being explored. Taq probing could be used to detect numerous other SNP associated with bacterial antibiotic resistance.

\section{F6}

\section{DEVELOPMENT OF MOLECULAR BIOLOGY AND BIOINFORMATICS TOOLS FOR THE IDENTIFICATION OF FUNGAL PATHOGENS IN A REFERENCE LABORATORY SETTING}

\section{H CHAREST, R CANTIN, G ST-GERMAIN}

Laboratoire de santé publique du Québec/INSPQ, Sainte-Anne-deBellevue, QC

BACKGROUND: The Mycology reference laboratory of the Laboratoire de santé publique du Québec receives approximately 1800 fungal clinical isolates per year for identification (ID). Although conventional phenotypic methods are sufficient to identify $>95 \%$ of strains, this approach has limitations, especially when dealing with atypical, nonsporulating, or newly described organisms.

OBJECTIVES: Develop and include DNA-based methods in the ID algorithms to improve the accurate identification of medically important fungi.

METHODS: Published PCR amplification and sequencing assays targeting ribosomal (D1/D2, ITS, and IGS1) and $\beta$-tubulin coding genes were gradually introduced into the fungal ID scheme. Reference strain sequences determined in-house or extracted from selected publications were used to create local databases of fungal pathogens. Bioinformatics tools were designed to manage workflow, integrate conventional method and DNAbased results within the same platform, compile DNA sequences and allow direct access to local or public databases.

RESULTS: Over 2000 sequencing reactions were performed to characterize 241 reference strains and 495 clinical isolates. The selection of DNA targets for each isolate was based on preliminary results obtained by conventional methods. DNA sequencing results allowed the conclusive identification of 391 (79\%) clinical isolates encompassing 81 genera and 134 species. Aspergillus spp. (113 strains, 21 species), Candida spp. (88 strains, 15 species) and dermatophytes (57 strains, 11 species) represented $52 \%$ of the isolates analyzed by sequencing.

CONCLUSION: The addition of sequencing tools to conventional identification algorithms in our reference laboratory has had a major impact on our ability to reliably identify difficult isolates and keep abreast of taxonomic changes.

\section{ROOM: VILLE-MARIE B}

\section{SESSION G}

\section{G1}

\section{DETECTION OF VARICELLA-ZOSTER VIRUS (VZV) FROM GENITAL SPECIMENS}

$\underline{A ~ M A K}^{1}, M_{\text {PETRIC }}^{1,2}, M_{\text {KRAJDEN }}^{1,2}$, P TANG $^{1,2}$

${ }^{1}$ PHSA Laboratory, BCCDC; ${ }^{2}$ University of British Columbia, Vancouver, BC

OBJECTIVE: After introduction of a multiplex herpes simplex virus (HSV) $1 \& 2$ and VZV PCR assay, VZV DNA was detected from genital lesions. A study was initiated to determine the frequency of VZV detection in genital lesion specimens relative to HSV.

METHODS: A multiplex PCR assay, adapted from Weidemann et al, 2003 was validated and implemented as reported previously (Eisler D. et al, CACMID-2010). A review of the viruses detected at various anatomical sites from May 1 to Aug 31, 2010 was performed and compared to the same period in 2009 during which centrifugal inoculation into shell vials followed by IF microscopy was used for HSV viral detection.

RESULTS: Implementation of multiplex HSV/VZV PCR increased overall positivity to $43.1 \%(2741 / 6356)$ of all specimens in 2010 compared with shell vial based HSV detection in $23.1 \%(1653 / 6944)$ in 2009. In 2010, 44.6\% (2838/6356) of tested specimens were from a genital source. Among genital samples HSV-1 was detected in 41.8\%, (541/1294), HSV-2 in $55.9 \%$ (718/1294) and VZV was detected in 2.7\% (35/1294). VZV was detected in genital samples in all age groups except in the first decade of life.

CONCLUSIONS: Multiplex PCR for HSV 1 \& 2, and VZV increases detection of HSV/VZV. Genital VZV infections occur in approximately $2.7 \%$ of HSV $1 \& 2 /$ VZV reactive genital specimens and likely represent a manifestation of shingles. Detection of genital VZV will have implications on patient counselling, the risk of recurrence and transmission and might impact treatment. 
G2

INVESTIGATION OF A HUMAN RHINOVIRUS OUTBREAK IN A CANADIAN LONG-TERM CARE FACILITY

S MUBAREKA ${ }^{1,2}$, L LOUIE ${ }^{1}$, O YOW ${ }^{1}$, A GRANADO ${ }^{3,4}$, K LUINSTRA $^{3}$, H WONG $^{1}$, A ARORA ${ }^{1}$, S CHONG ${ }^{3}$, A PETRICH ${ }^{5}$, M VEARNCOMBE ${ }^{1,2}$, A SIMOR ${ }^{1,2}$, J MAHONY ${ }^{3,4}$, M SMIEJA $^{3,4}$

${ }^{1}$ Sunnybrook Health Sciences Centre and Research Institute; ${ }^{2}$ University of Toronto, Toronto; ${ }^{3} \mathrm{St}$ Joseph's Hospital; ${ }^{4} \mathrm{McMaster}$ University, Hamilton; ${ }^{5}$ The Hospital for Sick Children, Toronto, ON OBJECTIVES: Outbreaks of respiratory illness in long-term care facilities due to human rhinovirus (HRV) have been well-described. Rapid evolution of HRV variants has been observed in experimental human infection. Our primary objective was to gain an understanding of the molecular epidemiology of HRV during a large outbreak of respiratory illness in a longterm care facility (LTCF).

METHODS: Active surveillance was undertaken whereby nasopharyngeal swabs were collected from LTCF residents with one or more of the following: fever, cough, rhinitis, or congestion. Multiplex PCR using the Resplex II version 2 assay was performed on specimens, and samples positive for HRV were then submitted for partial sequence analysis of the 5' untranslated (UTR) and VP1 capsid regions.

RESULTS: Fifty-nine residents were positive for HRV. The average age was 88.7 years and $94.9 \%$ were male. Nineteen $(32.2 \%)$ received antibiotics and 17 (28.8\%) underwent chest radiography. Two residents died after transfer to an acute care setting for respiratory distress. Seven units in 2 wings of the LTCF were affected, resulting in 3152.5 resident unit closure days. Five different HRV strains were identified by 5'UTR sequencing, and 4 strains by VP1 sequencing. An HRV serotype 1B-like virus dominated ( $\mathrm{n}=48$ by $5^{\prime}$ UTR and $\mathrm{n}=51$ by VPI).

CONCLUSION: Multiple strains of HRV were implicated in this LTCF respiratory outbreak. One HRV strain dominated, warranting further investigation into viral determinants for virulence and transmission.

\section{G3}

COMPREHENSIVE MICROBIOLOGICAL ANALYSIS OF FATAL CASES OF PANDEMIC INFLUENZA A VIRUS (H1N1)

S PATEL ${ }^{1,2}$, A MARCHAND-AUSTIN ${ }^{1}, 3$, J LONGTIN ${ }^{1}$, E LOMBOS ${ }^{1}$, P TANG $^{1}$, P RAWTE ${ }^{1}$, D LOW ${ }^{1,2,4}$, J GUBBAY ${ }^{1,2,4}$, F JAMIESON $^{1}$

${ }^{1}$ Ontario Agency for Health Protection and Promotion; ${ }^{2}$ University of Toronto, Toronto, ON; ${ }^{3}$ Public Heatlh Agency of Canada, Winnipeg, MB; ${ }^{4}$ Mount Sinai Hospital, Toronto, ON

OBJECTIVES: In previous influenza pandemics, mortality was attributed to secondary bacterial pneumonia. We provide a summary of microbiological findings on autopsy specimens submitted during the 2009 pandemic. METHODS: Specimens from respiratory deaths were submitted for pH1N1 testing. Nasopharyngeal (NP) swabs were tested for influenza A by real-time Flu A PCR; positive specimens were sub-typed for the pandemic strain. A multiplex viral panel (MVP) was used to detect respiratory viruses. Specimens were also tested for Mycoplasma and Chlamydophila spp using PCR. Respiratory specimens (BAL, lungs, trachea) were also set-up for CMV shell viral culture and bacterial cultures including Legionella spp. Molecular assays were performed to detect Staphylococcus aureus and Streptococcus pneumoniae.

RESULTS: We received autopsy specimens from 92 respiratory deaths. Twenty-two patients were positive for pH1N1. Of 11 patients with respiratory tissue specimens submitted, 8 were positive for $\mathrm{pH} 1 \mathrm{~N} 1$. Bacterial organisms were detected in 5 of 7 respiratory tissue specimens: S aureus (3 patients), S pneumoniae (1), and Group B streptococci (1). CONCLUSIONS: Most tissue specimens from pH1N1 positive also had bacterial organisms, predominantly $S$ aureus. Despite testing guidelines, only a small proportion of autopsies had multiple specimens submitted, making it difficult to evaluate the role of $\mathrm{pH} 1 \mathrm{~N} 1$ in contributing to fatality. A more concerted approach involving microbiologists and pathologists is essential when investigating new pathogens.

\section{G4}

WHY DID HEALTH CARE WORKERS REFUSE THE PH1N1 VACCINE DURING THE PANDEMIC?

K CORACE ${ }^{1}$, C PREMATUNGA ${ }^{1}$, A MCCARTHY ${ }^{1}, \mathrm{~V}^{\text {ROTH }}{ }^{1}$, T HAYES $^{2}$, KN SUH ${ }^{1}$, L BALFOUR $^{1}$, G GARBER ${ }^{1}$

${ }^{1}$ The University of Ottawa at The Ottawa Hospital; ${ }^{2}$ The Ottawa Hospital, Ottawa, ON

OBJECTIVES: Maintaining the health and availability of Health Care Workers (HCWs) is an essential component of pandemic preparedness. A key to protecting HCWs is the pH1N1 flu vaccination. However, the number of HCWs who get the flu vaccine falls well below the public health target of $90 \%$. Last year a mass $\mathrm{pH} 1 \mathrm{~N} 1$ vaccination campaign was launched at our hospital, yet $30 \%$ of staff refused vaccination. Why do HCWs refuse flu vaccinations? This study examined the motivators and barriers influencing pH1N1 vaccination among HCWs in order to design and implement a more effective vaccine campaign that addresses these barriers.

METHODS: This study was conducted at one of the largest tertiary care hospitals in Canada. Information was compiled on staff vaccine uptake during the pH1N1 vaccination roll-out, and Occupational Health and Safety housed a list of employees' vaccination status. Using this list, staff were recruited by mass mail out of a survey, and 3260 participants (2848 who received the pH1N1 vaccine and 412 who refused) completed measures of sociodemographics, flu vaccine history, $\mathrm{pH} 1 \mathrm{~N} 1$ vaccine knowledge and attitudes, and motivation for vaccine uptake or refusal.

RESULTS: Independent sample $t$-tests revealed that compared to HCWs who received the vaccine, HCWs who refused the vaccine were less likely to believe that (1) the vaccine was safe, (2) the vaccine would prevent themselves, family members and patients from getting $\mathrm{pH} 1 \mathrm{~N} 1$, and (3) they were at risk of pH1N1 $(p<.001)$. HCWs who refused vaccination were less likely to report that their supervisors, physicians, and colleagues encouraged them to get vaccinated $(p<.001)$. No significant differences were found between vaccine accepters and refusers in terms of fear of needles, not having time to receive vaccination, or disliking injections ( $p>.05$ ). Vaccine refusers identified lack of information about the vaccine as a key barrier to uptake.

CONCLUSIONS: Results identified fundamental reasons why a core group of HCWs refused vaccination and provide direction for future vaccination campaigns. Creating a culture of vaccine promotion in the workplace, characterized by strong messaging from HCWs supervisors and colleagues to enhance vaccine uptake is important. This work will impact the local and national pandemic vaccine and other influenza vaccine campaign strategies and implementations targeted at HCWs; thus, helping HCWs stay healthy and reducing the impact of influenza on health care organizations and patients.

G5

SELF-REPORTED INFLUENZA VACCINATION, ILLNESS AND ABSENTEEISM AMONG CANADIAN HEALTHCARE WORKERS DURING THE 2009 H1N1 INFLUENZA PANDEMIC

R MITCHELL ${ }^{1}$, G ASTRAKIANAKIS ${ }^{2}$, E BRYCE ${ }^{3}$, R GERVAIS ${ }^{1}$, D GRAVEL $^{1}$, L JOHNSTON ${ }^{4}$, S LEDUC ${ }^{1}$, V ROTH ${ }^{5}$, G TAYLOR ${ }^{6}$, M VEARNCOMBE${ }^{7}$, K WILKINSON ${ }^{1}$, Canadian Nosocomial Infection Surveillance Program ${ }^{1}$

${ }^{1}$ Centre for Communicable Diseases and Infection Control, Public Health Agency of Canada, Ottawa, ON; ${ }^{2}$ University of British Columbia; ${ }^{3}$ Vancouver General Hospital, Vancouver, BC; ${ }^{4}$ Queen Elizabeth II Health Sciences Centre, Halifax, NS; ${ }^{5}$ The Ottawa Hospital, Ottawa, ON; ${ }^{6}$ University of Alberta Hospital, Edmonton, AB; ${ }^{7}$ Sunnybrook Health Sciences Centre, Toronto, ON OBJECTIVES: To determine pandemic H1N1 (pH1N1) and seasonal influenza vaccination coverage; to explore reasons for vaccine declination; and to measure self-reported illness and absenteeism due to influenza-like illness (ILI) in Canadian HCWs.

METHODS: In February 2010 a self-administered survey was distributed to HCWs who had been involved in the direct care of patients on pH1N1 precautions in ten Canadian acute care facilities. 
RESULTS: Surveys were returned from $979 \mathrm{HCW}$; $80 \%$ were nurses, $14 \%$ respiratory therapists (RT), and $6 \%$ physicians. Eighty-three per cent indicated they had received the $\mathrm{pH} 1 \mathrm{~N} 1$ vaccine. A significantly higher proportion of physicians than nurses reported $\mathrm{pH} 1 \mathrm{~N} 1$ vaccination $(94 \%$ vs. $81 \% ; \mathrm{p}=0.014)$. There were 372 respondents $(38 \%)$ who reported receipt of the 2009-10 seasonal influenza vaccine. Physicians were significantly more likely to report seasonal vaccination than both nurses $(59 \%$ vs. $37 \%$; $=0.0006)$ and RT ( $59 \%$ vs. $36 \% ; p=0.002$ ). The most common reasons for refusing the seasonal vaccine were concerns over vaccine safety (24\%) and preference for natural immunity $(35 \%)$. Thirty per cent of unvaccinated $\mathrm{HCW}$ s reported ILI compared to $23 \%$ of vaccinated HCWs $(\mathrm{p}=0.03)$, however there was no difference in absenteeism.

CONCLUSION: Self-reported vaccine coverage for $\mathrm{pH} 1 \mathrm{~N} 1$ was higher than coverage reported worldwide. Self-reported seasonal vaccine coverage was low, which may reflect challenges in the acceptance of multiple vaccines during a pandemic season.

\section{G6}

REPORTED USE OF PERSONAL PROTECTIVE EQUIPMENT (PPE) AMONG CANADIAN HEALTHCARE WORKERS (HCWS) DURING THE SECOND WAVE OF THE H1N1 INFLUENZA PANDEMIC

R MITCHELL ${ }^{1}$, G ASTRAKIANAKIS ${ }^{2}$, E BRYCE ${ }^{3}$, R GERVAIS $^{1}$, D GRAVEL $^{1}$, L JOHNSTON ${ }^{4}$, S LEDUC ${ }^{1}$, V ROTH ${ }^{5}$, G TAYLOR $^{6}$, M VEARNCOMBE ${ }^{7}, C^{2}$ WEIR ${ }^{1}$, Canadian Nosocomial Infection Surveillance Program ${ }^{1}$

${ }^{1}$ Centre for Communicable Diseases and Infection Control, Public Health Agency of Canada, Ottawa, ON; ${ }^{2}$ University of British Columbia; ${ }^{3}$ Vancouver General Hospital, Vancouver, BC; ${ }^{4}$ Queen Elizabeth II Health Sciences Centre, Halifax, NS; ${ }^{5}$ The Ottawa Hospital, Ottawa, ON; ${ }^{6}$ University of Alberta Hospital, Edmonton, $\mathrm{AB},{ }^{7}$ Sunnybrook Health Sciences Centre, Toronto, $\mathrm{ON}$

OBJECTIVES: To determine PPE use among Canadian HCWs involved in the care of pandemic H1N1 patients between September and December 2009. METHODS: A self-administered survey was distributed to HCWs in ten Canadian acute care facilities in February 2010.

RESULTS: Surveys were returned from $979 \mathrm{HCW}$; 80\% were nurses, $14 \%$ respiratory therapists, and $6 \%$ physicians. The majority of respondents reported receiving sufficient training on when (90\%) and how (92\%) to use PPE, as well as which PPE to use $(84 \%)$, and $82 \%$ reported that all PPE were consistently available at point of care.

$\mathrm{HCW}$ reported always wearing gloves (87\%), gowns (75\%), N95 respirators $(70 \%)$ and protective eyewear $(61 \%)$ when performing an aerosolgenerating medical procedure (AGMP). More nurses and respiratory therapists (67\% and $72 \%$ respectively) reported always using N95 respirators when performing an AGMP than physicians (43\%) ( $\mathrm{p}=0.0083$ and $\mathrm{p}=0.0045$ respectively). HCWs in the intensive care and designated influenza wards reported significantly higher compliance with N95 respirator and eyewear use than the emergency (ER) unit (e.g. $42 \%$ of HCWs in the influenza ward vs. $23 \%$ in the ER reported always performing a user seal check after putting on an N95 respirator, $\mathrm{p}<0.004$ ).

CONCLUSION: HCWs reported that PPE was readily available and that they received sufficient training in its usage. However, PPE use was suboptimal and significant differences exist between professional groups and clinical areas which may impact nosocomial transmission of influenza.
ROOM: ST-ANTOINE B

\section{SESSION H}

\section{H1}

EXTENSIVE GENOMIC VARIATION WITHIN CLONAL
COMPLEXES OF NEISSERIA MENINGITIDIS

W HAO ${ }^{1,2}$, J MA ${ }^{1}$, K WARREN ${ }^{1}$, P TSANG $^{3}$, D LOW ${ }^{1,2}$,

F JAMIESON ${ }^{1,2}$, D ALEXANDER ${ }^{1,2}$

${ }^{1}$ Ontario Agency for Health Protectino and Promotion, Public Health Laboratories; ${ }^{2}$ University of Toronto, Department of Laboratory Medicine and Pathobiology, Toronto, ON; ${ }^{3}$ Public Health Agency of Canada, National Microbiology Laboratory, Winnipeg, MB

OBJECTIVE: Invasive meningococcal disease (IMD) is a complex and potentially fatal bacterial infection caused by Neisseria meningitidis. Current vaccines only provide protection against a subset of meningococcal serogroups. In this study, a comparative genomics approach was used to analyze virulence genes in isolates from clinically important clonal complexes. The objective was to identify IMD virulence loci as well as candidates for an improved, broadly protective meningococcal vaccine.

METHODS: Genome sequences for seven clinical N.meningitidis strains (6 invasive blood isolates, 1 non-invasive isolate) were generated via Roche GS-FLX pyrosequencing. Genome sequences for 30 additional Neisseria strains were obtained from public databases. Multi-locus sequence types (STs) were assigned using a standard 7-loci scheme. A set of 164 putative virulence loci was derived from published literature. Orthologs of these virulence genes were identified using BLASTN. MUSCLE was used to align sequences and compare orthologs from different strains. The phylogenetic incongruence of the virulence loci was assessed with the Approximate Unbiased (AU) test.

RESULTS: The 37 Neisseria isolates included serogroup A, B, C, Y, W135 and non-encapsulated strains and represented 15 clonal groups. Analysis of 164 virulence loci revealed extensive genomic variation among these strains. In pairs of strains with identical ST, up to $30 \%$ of orthologous genes were different. The AU test indicated that, for the majority of virulence loci, phylogenetic topologies were statistically different. Furthermore, only 20 of the 164 virulence genes were found to be conserved or slowly evolving.

CONCLUSIONS: Previous studies have shown associations between IMD and specific, hyper-virulent N. meningitidis STs. However, our work shows that the level of genomic diversity within clonal complexes has been underestimated. In strain pairs with identical STs, up to 30\% of 164 virulence genes are different. AU analysis indicates that the genes had multiple, distinct evolutionary origins. Only a subset of 20 loci are identified as conserved or slowly evolving. The limited sequence variation of these genes suggests that the loci, including pili and cell-wall associated proteins, play key roles in IMD and may be viable candidates for a new, broadly protective meningococcal vaccine.

\section{$\mathrm{H} 2$}

WHOLE GENOME ANALYSES AND EPIDEMIOLOGICAL ASPECTS OF A PROPOSED NEW EPIDEMIC CLONE OF LISTERIA MONOCYTOGENES IN CANADA

A REIMER ${ }^{1}$, M WALKER ${ }^{1}$, S TYLER ${ }^{1}$, J ZIEGLER ${ }^{1,2}$, C SINGH $^{1}$, P HEMMONS ${ }^{1,3}$, C CROSS ${ }^{1}$, M GRAHAM ${ }^{1}$, G VAN DOMSELAAR ${ }^{1}$, V ALLEN $^{4}$, F PAGOTTO ${ }^{5}$, M GILMOUR ${ }^{1,2}$

${ }^{1}$ Public Health Agency of Canada; ${ }^{2}$ University of Manitoba, Winnipeg, MB; ${ }^{3}$ Thompson Rivers University, Kamloops, BC; ${ }^{4}$ Ontario Agency for Health Protection and Promotion, Toronto; ${ }^{5}$ Health Canada, Ottawa, ON

OBJECTIVE: Retrospective analysis of Canadian listeriosis cases (19882010) revealed a significant proportion of serotype $1 / 2 a$ isolates sharing indistinguishable or highly-related PFGE patterns. Notably, this subtype emerged in human clinical cases as early as 1988, was responsible for the 2008 nationwide 
listeriosis outbreak, and continues to be detected amongst current clinical clusters and sporadic cases. To investigate whether a new epidemic clone (EC) has emerged in Canada, we characterized a geographically and temporally diverse panel of Canadian L.monocytogenes $(\mathrm{Lm})$ isolates.

METHODS: We used MLST to assess the genetic relatedness of 132 Canadian isolates, representing all $\mathrm{Lm}$ serotypes and a variety of PFGE patterns, 69 of which represented the putative EC. Of these, 4 epidemiologically unrelated isolates, collected over 12 years, underwent definitive characterization using whole-genome sequencing (WGS).

RESULTS: MLST delineated the putative EC into 4 sequence types (ST). Two highly related STs predominated. WGS confirmed a high degree of genetic relatedness between suspected EC isolates. Evolutionary relationships between these strains were characterized by analyses of SNP loci (14 to 56 SNPs) and prophage indels or replacements. Most EC isolates share horizontally transferred genetic elements encoding putative determinants for novel secretion systems and sanitizer resistance.

CONCLUSION: This study has confirmed that a new Lm serotype $1 / 2 \mathrm{a}$ EC has emerged within Canada. WGS of additional EC isolates is required to fully characterize EC strain genetic relationships and evolution.

\section{H3}

PROVISIONAL TAXON, CDC GROUP NO-1, REPRESENTS A NEW GENUS IN THE FAMILY COMAMONADACEAE, LINKED TO ZOONOTIC-BASED WOUNDS / OTHER INFECTIONS IN HUMANS

\section{$\underline{\text { K BERNARD }}^{1,2}$, T BURDZ $^{1}$, B NG $^{1}$, AL PACHECO $^{1}$, D WIEBE $^{1}$, A REIMER ${ }^{1}$}

${ }^{1}$ National Microbiology Laboratory PHAC; ${ }^{2}$ University of Manitoba Department of Medical Microbiology, Winnipeg, MB

OBJECTIVES: A group of Gram negative rods from our collection originally identified as CDC group NO-1, were studied. Strains had been collected over $\sim 30 \mathrm{y}$, recovered from either wounds linked to dog or cat bites, cellulitis (zoonotic contact unknown), or peritoneal dialysate fluid. CDC group NO-1 has no formal taxonomic status, nor has genetic characterization been published which would permit possible assignment to bacterial family, genus and species. Therefore, a polyphasic approach was used on NML strains to a) determine if the group was monophyletic and b) to ascertain an appropriate taxonomic position for these isolates.

METHODS: A polyphasic approach was used, consisting of standard phenotypic, chemotaxonomic and 16S rRNA gene sequencing methods. RESULTS: 6 strains from 5 patients (NML 79-0751, 91-0035, 91-0036, 97-0112, 00-0135 and 03-0146) were found to form a monophyletic taxon group, with $99.4 \%$ or greater similarity to each other by $16 \mathrm{~S}$. One additional strain, 97-0147, was found to be an outlier (97\%) to the others. Strains were otherwise closest to the genus Lampropedia (95\% similarity) with more distant relationships to other genera in the family Comamonadaceae. Cellular fatty acid composition, menaquinones and $\mathrm{G}+\mathrm{C}$ mol\% were consistent with this family. All strains were aerobic Gram negative rods or coccobacilli, catalase positive, oxidase variable, non-motile, nitrate reducing, but otherwise, non reactive towards a variety of sugars / substrates.

CONCLUSIONS: 6 CDC group NO-1 like strains represent a single taxon group suggestive of a genus and species novum in the family Comamonadaceae. NML 97-0147 represents a phenotypically similar but separate taxon.

\section{H4}

\section{MULTI-LOCUS SEQUENCE TYPING OF COASTAL PACIFIC} NORTHWEST CRYPTOCOCCUS ISOLATES

\section{M-K LEE, S MAN, A BALBIRNIE, S MITHANI, Q WONG, L HOANG,} MG MORSHED

British Columbia Centre for Disease Control Public Health and Reference Laboratory (BCPHL), Vancouver, BC

BACKGROUND: This study evaluated the role of multi-locus sequence typing (MLST) for speciation as well as its usefulness in determining molecular epidemiological trends in Cryptococcus isolated from humans, animal and environmental samples from British Columbia and the Pacific Northwest.
METHODS: Cryptococcus spp. $(\mathrm{n}=107)$ were isolated using CGB agar media from clinical, veterinarian and environmental samples. Restriction Fragment Length Polymorphism (RFLP) targeting the URA5 gene was carried out. MLST targeted 7 housekeeping genes, the CAP59, GPD1, LAC1, PLB1, SOD1, URA5 and IGS1 loci.

RESULTS: Compared to the previously established RFLP method, MLST was able to identify all RFLP genotypes of C. gattii and provided higher typing resolution. MLST further typed the RFLP genotype VGI into 7 MLST sequence types, VGIIa into 3, VGIIb in to 2, and VGIII into 2. MLST Sequence type VGIIa_1 was the predominate strain found in the Pacific Northwest, representing over $70 \%$ of the human, animal, and environment samples tested in this study.

CONCLUSION: There was a perfect correlation between RFLP and MLST for the identification of C. gattii and as expected, MLST demonstrated higher subtyping resolution. Despite the expense and time-consuming nature of MLST, it provides greater detail, allowing epidemiological studies that would not be possible with RFLP. The majority of the isolates from this region was genotype VGIIa (RFLP) and sequence type VGIIa_1 (MLST). This demonstrates the clonal nature of C. gattii, particularly in B.C.

\section{H5}

\section{PRELIMINARY EVIDENCE OF CACHE VALLEY VIRUS INFECTIONS AND ASSOCIATED HUMAN ILLNESS IN WESTERN CANADA IN 2009}

K DIMITROVA ${ }^{1}$, M ANDONOVA ${ }^{1}$, K MAKOWSKI ${ }^{1}$, K HOLLOWAY ${ }^{1}$, PN LEVETT $^{3}$, K KADKHODA ${ }^{2}$, P VAN CAESEELE $^{2}$, MA DREBOT ${ }^{1}$

${ }^{1}$ Public Health Agency of Canada; ${ }^{2}$ Cadham Provincial Laboratory, Winnipeg, MB; ${ }^{3}$ Saskatchewan Disease Control Laboratory, Regina, SK OBJECTIVE: Cache Valley virus (CVV) is a mosquito-borne virus belonging to the family Bunyaviridae, genus Orthobunyavirus that is widespread throughout North America. The virus has been documented to cause congenital defects in livestock but two cases of CVV-associated neurological disease in humans have been reported in the United States. The objective of this study was to screen subsets of patient sera originally collected in Manitoba (MB) and Saskatchewan (SK) for West Nile virus (WNV) testing for the presence of CVV antibody.

METHODS: Aliquots of sera from individuals (MB and SK residents) previously suspected to be WNV cases but testing negative for WNV during the 2009 mosquito season were utilized in this study. Testing of human sera for CVV antibody was carried out by plaque reduction neutralization tests (PRNT). CVV was propagated in Vero E6 cells and a viral plaque titration was carried out to determine the viral titre per ml. A constant viral dose of 100 plaque forming units was added to serially diluted sera in a PRNT assay to identify CVV specific antibodies.

RESULTS: 216 WNV suspect-case sera from SK were initially screened by PRNT at a titre of 1:20 with $9(5 \%)$ of the sera giving CVV specific neutralizing titres of $\geq 1: 20$. Sera were further end point titrated with several samples exhibiting significant titres of 40-80 to CVV. An initial testing of 55 sera from WNV suspect cases in MB identified 9 (16\%) patients with CVV antibodies indicating a significant level of virus exposure in this province as well.

CONCLUSION: The application of serological procedures to identify probable CVV infections in symptomatic patients provides preliminary evidence that this pathogen may be contributing to a certain level of exposures and possible illness among patients in MB and SK. Additional surveillance and diagnostic testing is warranted to verify if CVV is associated with disease not only in western Canada but other regions within the country.

\section{H6}

\section{THE IDENTIFICATION OF PROBABLE CASES OF CALIFORNIA SEROGROUP VIRUS INFECTIONS IN MANITOBA IN 2010}

K MAKOWSKI ${ }^{1}$, K DIMITROVA ${ }^{1}$, M ANDONOVA ${ }^{1}$, K HOLLOWAY $^{1}$, $\mathrm{K}$ KADKHODA $^{2}$, P VAN CAESEELE $^{2}$, MA DREBOT ${ }^{1}$

${ }^{1}$ Public Health Agency of Canada; ${ }^{2}$ Cadham Provincial Laboratory, Winnipeg, $\mathrm{MB}$

OBJECTIVE: Snowshoe hare virus (SSHV) and Jamestown Canyon virus (JCV) are mosquito-borne viruses that are members of the California 
serogroup of agents belonging to the family Bunyaviridae, genus Orthobunyavirus. These viruses are found throughout North America and have been associated with disease ranging from febrile illness to encephalitis. The objective of this study was to test patient sera collected in Manitoba (MB) during the 2010 mosquito season for the presence of IgM and IgG antibody to JC and SSH viruses and provide a preliminary assessment of the possible burden of illness contributed by these agents.

METHOD: Aliquots of sera from MB individuals previously suspected to be WNV cases but testing negative for WNV during the 2010 mosquito season were utilized in this study. Testing of human sera for SSH and JC virus antibody was carried out by differential IgM ELISAs and plaque reduction neutralization tests (PRNT) utilizing California serogroup antigens and titrated virus - serum mixtures.

RESULTS: A total of 626 sera from MB patients collected during the mosquito season (May - October ) were screened by both PRNT methodology and IgM ELISAs. 156 (25\%) of the sera were found to be positive for virus specific antibody (primarily $\operatorname{IgG}$ ) by neutralization to at least one of the two California serogroup viruses being studied. Out of the 366 sera that have been tested to date by IgM ELISA \& PRNT, 36 (10\%) have shown to be seropositive for both viral IgM and neutralizing antibody indicative of a probable case designation.

CONCLUSIONS: The results indicate that $25 \%$ of targeted MB patients (suspect WNV cases) were seropositive for California serogroup viruses when tested for virus specific antibody in 2010. Of these a significant number of individuals also had IgM antibody to SSH and/or JC viruses which correlates with possible cases of illness associated with these pathogens.

SATURDAY, APRIL 9 ROOM: VILLE-MARIE A

\section{SESSION I}

\section{I1}

\section{CHRONIC HEPATITIS E IN A CANADIAN HIV INFECTED PATIENT}

A ANDONOV $^{1}$, M POLIQUIN², L LIN', A MASON ${ }^{3}$, D MURPHY 2,4

${ }^{1}$ National Microbiology Laboratory, Public Health Agency of Canada, Winnipeg, MB; ${ }^{2} \mathrm{CHUM}$-Clinique Medicale L'Actuel, Montreal, QC; ${ }^{3}$ University of Alberta, Edmonton, AB; ${ }^{4}$ Laboratoire de sante publique du Quebec, Montreal, QC

OBJECTIVE: Hepatitis E virus (HEV) is a major cause of acute hepatitis in Asia and Africa where serious epidemics often occur. Sporadic HEV cases are being increasingly reported in industrialized countries. Recently chronic HEV infection has been observed in kidney or liver transplant recipients in Western Europe, which increased attention to HEV in the context of immunosuppressed patients. The objective of this study was to detect evidence of HEV infection in immunocompromised Canadian individuals.

METHODS: RT-PCR with four different primer sets targeting the ORF2 HEV genomic region were used for HEV RNA detection. Serologic testing was performed for IgG and IgM anti-HEV antibody with two different commercial kits. Forty-three samples collected six months apart from 21 liver transplant patients and $80 \mathrm{HIV}$ infected patients were tested.

RESULTS: No evidence of HEV infection was found in the immunosupressed liver-transplant patients. One HIV patient was found positive for HEV RNA on two occasions within 1 year period, fulfilling the criteria for chronic hepatitis E due to HEV RNA detection and elevated ALTs for more than six months. This is the first Canadian chronic HEV case and only the third reported in HIV patients worldwide. The HEV genotype in this patient was $3 \mathrm{a}$ which is in line with previous data in HIV patients all of whom were also infected with genotype 3, implicated in all autochthonous cases. Liver-induced injury due to anti-retroviral therapy is not uncommon; as the traditional culprits of liver disease coinfection with HIV are more often $\mathrm{HBV}$ and $\mathrm{HCV}, \mathrm{HEV} / \mathrm{HIV}$ coinfection may remain under diagnosed.
CONCLUSION: HEV RNA screening should be included in the laboratory diagnosis of liver disease in HIV immunocompromised patients due to inconsistent serology results.

\section{2}

SUCCESSFUL CARDIAC TRANSPLANTATION IN A 4-YEAR-OLD CHILD WITH ACTIVE PARAINFLUENZA-3 INFECTION: EXPERIENCE WITH SYSTEMIC RIBAVIRIN THERAPY

\section{KANII, W VAUDRY}

University of Alberta, Edmonton, AB

BACKGROUND: Parainfluenza virus can cause severe respiratory tract infections in haematology stem cell and solid organ transplant recipients, sometimes necessitating mechanical ventilation or causing death.

CASE: A 4-year-old girl with restrictive cardiomyopathy, awaiting cardiac transplant for more than one year, developed confirmed parainfluenza-3 (PIF-3) respiratory tract infection two days prior to a donor heart becoming available. As she had been waiting for her transplant for almost 2 years, it was felt that the benefit of accepting the heart exceeded the risks of worsening of her current parainfluenza infection. The patient subsequently underwent successful cardiac transplantation with intravenous ribavirin cover and other immune suppressing agents for her transplant with a good outcome. The ribavirin was continued for a total of two weeks. Persistent PIF-3 RNA was detected on two further weekly subsequent nasopharyngeal aspirates using the Luminex Diagnostics Respiratory Virus Panel nucleic acid amplification assay which became negative on week 3 post transplant. She required transfusion with four units of packed red cells during her transplant procedure and one further transfusion on the last day of ribavirin therapy. Aside from anemia, no other manifestations of ribavirin toxicity were noted. DISCUSSION: From our literature search, this is the first case of a cardiac transplant done while the patient had active parainfluenza infection. It illustrates that successful outcomes of transplantation can be achieved in the face of active respiratory infection with close post-operative monitoring and active extended therapy with ribavirin and monitoring for anemia.

\section{3}

\section{LABORATORY-CONFIRMED PANDEMIC H1N1 INFLUENZA IN HOSPITALIZED ADULTS - FINDINGS FROM THE CANADIAN NOSOCOMIAL INFECTION SURVEILLANCE PROGRAM (CNISP), 2009-10}

K WILKINSON $^{1}$, G TAYLOR ${ }^{2}$, D GRAVEL ${ }^{1}$, B AMIHOD ${ }^{3}$, C FRENETTE ${ }^{4}$, AJ MCGEER ${ }^{5}$, D MOORE $^{6}, \mathrm{KN} \mathrm{SUH}^{7}$, A WONG $^{8}$, R MITCHELL ${ }^{\prime}$

${ }^{1}$ Public Health Agency of Canada, Ottawa, ON; ${ }^{2}$ University of Alberta Hospital, Edmonton, $\mathrm{AB} ;{ }^{3}$ The Jewish General Hospital; ${ }^{4}$ McGill University Health Centre, Montreal, QC; ${ }^{5}$ Mount Sinai Hospital, Toronto, ON; ${ }^{6}$ Montreal Children's Hospital, Montreal, QC; ${ }^{7}$ The Ottawa Hospital, Ottawa, ON; ${ }^{8}$ Royal University Hospital, Saskatoon, SK

OBJECTIVES: To describe the epidemiology of laboratory-confirmed pandemic H1N1 (pH1N1) influenza in adult inpatients at participating Canadian hospitals between June 1, 2009 and May 31, 2010 and compare to previous years' seasonal surveillance.

METHODS: Case forms were completed for inpatients ( $>=16$ years) with influenza. Variables collected included ICU admissions and death attributed to influenza assessed 30 days after initial diagnosis.

RESULTS: Thirty-seven hospitals submitted forms for $701 \mathrm{pH} 1 \mathrm{~N} 1$ cases. The median age of the cases was 49 years (range 16 - 94). Vaccine history was available for 314 cases, and $21 \%(n=65)$ reported receiving vaccine. Oseltamivir was given to $90 \%$ of the cases a median of 3 days after symptom onset (range 0 - 24). Influenza-associated admission to ICU was required for $28 \%(n=197)$. The 30 day all-cause mortality was $7 \%$; influenza was the primary cause of 20 deaths and contributed to death in a further 22 cases for an influenza-attributed mortality of $6 \%$. The mean age at death was 50 years (SD 13.8).

CONCLUSIONS: The ICU admission rate and influenza-attributed mortality were similar to the three preceding influenza years, but the mean age 
at death was significantly younger during the pandemic $(p<0.01)$. Antivirals were prescribed for more patients with influenza during the pandemic $(90 \%)$ than in previous seasons $(35-47 \%)$. The $\mathrm{pH} 1 \mathrm{N1}$ virus appeared outside the traditional influenza season and impacted a different age group than seasonal viruses circulating in previous years, highlighting the importance of ongoing influenza surveillance.

\section{4}

\section{EPIDEMIOLOGICAL FEATURES AND PATHOGENS ASSOCIATED WITH MENINGITIS AMONG CHILDREN [0-15 YEARS]}

\section{S THLIZA $^{1}$, T LAWOYIN $^{2}$, I JALO $^{3}$}

${ }^{1}$ Hospitals Management Board Headquarters, Maiduguri, Borno State; ${ }^{2}$ Reproductive and Family Health Unit, Department of Community Medicine, College of Medicine, UCH, Ibadan, Oyo State; ${ }^{3}$ Department of Pediatrics, Federal Medical Centre, Gombe, Gombe State, Nigeria

OBJECTIVES: Meningococcal disease is contagious and is caused by the meningococcus. Epidemics can occur in any part of the world. However, the largest epidemics occur mainly in the semi-arid areas of sub-Saharan Africa, "the African meningitis belt." The disease is of public health importance with a huge health burden as morbidity and mortality is very high. In the tropics, especially the Sahel Savannah regions, this vaccinepreventable disease has left an indelible mark because of its havoc on the population. This study was designed to identify epidemiological features of meningitis and assess the microorganisms responsible for the disease over a twenty-year period (1983-2003) and also identify the risks and outcomes among pediatric age group (0-15 years).

METHODS: A case-control study was conducted where cerebrospinal meningitis is endemic as well as epidemic-prone. A structured questionnaire was used to collect information on socio-demographic and health status of 390 cases that were diagnosed from cerebrospinal fluid (CSF) and blood culture analysis. These were compared with records of 390 agematched controls admitted into hospital at the same time as the cases, and who did not have meningitis.

RESULTS: The majority of cases $(\mathrm{n}=288,73.8 \%)$ were under 5 years old and lived in high density areas [OR $=1.38(1.00-1.89), \mathrm{p}=0.040]$ where contact with someone with meningitis was significantly high. The bacterial isolates were predominantly Neisseria meningitidis which accounted for most cases $(n=202,52.7 \%)$ followed by Streptococcus pneumoniae $(n=87,22.9 \%)$, and $H$. influenza $(n=15,3.9 \%)$. On discharge $(n=22,5.8 \%)$ were newly diagnosed as deaf, this being the leading complication of meningitis. Hemiplegia and blindness were found in $(n=19) 5 \%$ each followed by cerebral palsy in $(n=18) 4.7 \%$ and seizure disorders in $(n=9) 2.4 \%$. Hydrocephalus, hypotonia and others accounted for $(n=7) 1.2 \%$. A high case fatality rate (CFR) of 32.7 deaths per 1,000 was associated with the disease.

CONCLUSION: Neisseria meningitidis accounted for most cases of the disease which is vaccine preventable. Efforts should be made to identify specific serotypes that may be epidemic prone in this area. Deafness was the leading complication of meningitis. Health education and vaccination of the populace will reduce the incidence of this disease.

\section{ROOM: ST-ANTOINE A}

\section{SESSION J}

\section{J1}

\section{EVALUATION OF WASP AUTOMATED MICROBIOLOGY SPECIMEN PROCESSOR FOR THE PROCESSING OF URINE SPECIMENS IN A HIGH VOLUME LABORATORY} N SHARMA ${ }^{1}$, H ALMOHRI $^{1}{ }^{12}$, S SMITH $^{1}$, C BECHARD $^{1}$, P ROMANO $^{1}$ ${ }^{1}$ LifeLabs Medical Laboratories, Toronto; ${ }^{2}$ Joseph Brant Memorial Hospital, Burlington, ON

OBJECTIVE: "WASP" is an automated microbiology processor. The objective is to evaluate the performance of WASP and assess the accuracy of plating, carryover and throughput for urine specimens in a community based high volume microbiology laboratory.

METHOD: This Study was conducted at LifeLabs Medical Laboratory in Ontario. A total of 607 urine specimens were processed by our current method and WASP. Of these, 588 specimens were used for the accuracy. The incubation time for both methods was $16-20 \mathrm{hrs}$. The reading of the culture plates was blinded. Positive cultures were defined by our laboratory protocol. The results were considered discordant if final results were clinically different. Throughput for WASP was determined by averaging the 11 runs. The carryover was determined by alternating known positive samples and sterile water.

RESULTS: There were total of 29 out of 588 (4.9\%) discordant results (none clinically significant). WASP throughput average was $40.3 \mathrm{sec} . / \mathrm{speci}-$ men or 89-100 plates per hour. There was no carryover. Technical errors were rare and easily resolved. 30-40 urine containers can be loaded on WASP at one time with walk-away time being 20 minutes.

CONCLUSION: The WASP streaking pattern was more consistent and provided more isolated colonies than our current process. The manual process is repetitive, error prone and may lead to repetitive motion injuries. We found the WASP to be user-friendly; it was easy to resolve technical errors.

\section{J2}

COMPARISON BETWEEN BD VIPER ${ }^{T M}$ XTR SYSTEM AND GENPROBE TIGRIS ${ }^{\circledR}$ DTS ${ }^{\circledR}$ SYSTEM FOR THE DETECTION OF CT/GC IN URINE SPECIMENS AND GENITAL SWABS H ALMOHRI $^{1,2}$, S BOSS ${ }^{1}$, S SMITH ${ }^{1}$, D SEALY $^{1}$, M KELLY $^{3}$, G RITCHIE $^{4}$, J BITTANTE 3 , M WONG 3

${ }^{1}$ LifeLabs Medical Laboratory Services, Toronto; ${ }^{2}$ Joseph Brant Memorial Hospital, Burlington; ${ }^{3}$ LifeLabs Medical Laboratory Services, Burnaby; ${ }^{4}$ Providence Health Care, Vancouver, BC

OBJECTIVE: To compare the performance of BD Viper XTR System and GenProbe Tigris DTS System in detecting CT and GC in urine samples and genital swabs from males and females.

METHODS: The study was conducted at LifeLabs Medical Laboratories in Ontario and British Columbia. Urine samples were collected in sterile containers. Swabs were collected on dry BD swabs in Ontario and GenProbe swabs in BC. All swabs and urine samples were tested using both systems. A third molecular method using Roche Amplicor was used for discrepant results. Sensitivities and specificities were calculated based on the Patient Infectivity Status. PIS is defined as positive if 2 molecular testing was positive.

RESULTS: Sensitivities and specificities for different specimens are given in the table:

\begin{tabular}{|c|c|c|c|c|}
\hline \multirow[b]{2}{*}{ Numbers } & \multicolumn{2}{|c|}{ BD Viper XTR } & \multicolumn{2}{|c|}{ GenProbe APTIMA Combo 2} \\
\hline & Sensitivity \% & Specificity $\%$ & Sensitivity\% & Specificity $\%$ \\
\hline $\begin{array}{l}\text { All specimens } \\
N=2179\end{array}$ & $\begin{array}{l}\text { CT: } 99.4, \mathrm{G} \\
97.7\end{array}$ & $\begin{array}{l}\text { CT: } 99.9, \text { GC: } \\
100\end{array}$ & $\begin{array}{l}\text { CT: 98.7, GC: } \\
100\end{array}$ & $\begin{array}{l}\text { CT: } 99.8, \mathrm{GC}: \\
100\end{array}$ \\
\hline $\begin{array}{l}\text { Swabs males } \\
N=73\end{array}$ & $\begin{array}{l}\text { CT:100, GC: } \\
100\end{array}$ & $\begin{array}{l}\text { CT: } 98.4, \text { GC: } \\
100\end{array}$ & $\begin{array}{l}\text { CT:100, GC: } \\
100\end{array}$ & $\begin{array}{l}\text { CT: } 98.4, \text { GC: } \\
100\end{array}$ \\
\hline $\begin{array}{l}\text { Swabs } \\
\text { females }\end{array}$ & $\begin{array}{l}\text { CT: } 100, \mathrm{GC}: \\
66.7\end{array}$ & $\begin{array}{l}\text { CT: 100, GC: } \\
100\end{array}$ & $\begin{array}{l}\text { СT: } 100, \mathrm{GC}: \\
100\end{array}$ & $\begin{array}{l}\text { СТ: } 99.9, \mathrm{GC}: \\
100\end{array}$ \\
\hline$=998$ & & & & \\
\hline $\begin{array}{l}\text { Urine males } \\
\mathrm{N}=678\end{array}$ & $\begin{array}{l}\text { CT: } 97.9, \mathrm{GC}: \\
100\end{array}$ & $\begin{array}{l}\text { CT: 100, GC: } \\
100\end{array}$ & $\begin{array}{l}\text { CT: 100, GC: } \\
100\end{array}$ & $\begin{array}{l}\text { CT: } 99.8, \mathrm{GC}: \\
100\end{array}$ \\
\hline $\begin{array}{l}\text { Urine females } \\
N=430\end{array}$ & $\begin{array}{l}\text { CT: 100, GC: } \\
100\end{array}$ & $\begin{array}{l}\text { СT: 100, GC: } \\
100\end{array}$ & $\begin{array}{l}\text { CT: } 90.9, \text { GC: } \\
100\end{array}$ & $\begin{array}{l}\text { CT: 100, GC: } \\
100\end{array}$ \\
\hline
\end{tabular}

CONCLUSION: Both methods are very similar in detecting CT/GC. Sensitivity of BD XTR for GC in female swab samples was lower at $66.7 \%$ $(2 / 3)$. This can be explained by low number of positive swabs for GC in this study. 


\section{J3}

MULTI-SITE EVALUATION OF TWO ENZYME IMMUNOASSAYS FOR VEROTOXINS (SHIGATOXINS) IN CLINICAL STOOL SAMPLES

L HOANG $^{1,2}$, D CARRANZA ${ }^{3}$, M CASTRO ${ }^{1}$, J DALY ${ }^{4,5}$,

B HOLTSLANDER ${ }^{3}$, S MALOTT ${ }^{4}$, A MAZZOCCO $^{3}$, A PACCAGNELLA ${ }^{1}$, SE RICHARDSON ${ }^{6}$, T WONG $^{1}$, R JOHNSON $^{3}$ ${ }^{1}$ BCCDC Public Health and Reference Microbiology Laboratory, PHSA; ${ }^{2}$ UBC Department of Pathology and Laboratory Medicine, Vancouver, BC; ${ }^{3}$ Laboratory for Foodborne Zoonoses, Public Health Agency of Canada, Guelph, ON; ${ }^{4}$ Microbiology Laboratories Primary Children's Medical Center; ${ }^{5}$ Department of Pathology, University of Utah, Salt Lake City, UT, United States; ${ }^{6}$ Division of Microbiology, The Hospital for Sick Children, Toronto, ON OBJECTIVES: Following recent best practice recommendations that all stools submitted for E. coli O157 testing also be screened for shigatoxin (STX), we performed an evaluation of the new Prolisa EHEC EIA and the Premier EHEC EIA for STX with the Vero cell cytotoxicity assay (VCA) as the reference method.

METHOD: Stool samples $(\mathrm{N}=525)$ collected prospectively in $2008-9$ by two paediatric hospitals and a provincial public health laboratory were tested directly and after broth enrichment by the two EIAs and compared to the VCA of filtrates of enrichment cultures.

RESULTS: Analysis of the two EIAs relative to VCA of enrichment cultures.

\begin{tabular}{lccccccc} 
& \multicolumn{3}{c}{ Direct Stool } & & \multicolumn{3}{c}{ Enriched Stool } \\
\cline { 2 - 3 } \cline { 6 - 7 } & Prolisa & Premier & P-value & & Prolisa & Premier & P-value \\
\hline Sensitivity & $45.9 \%$ & $43.2 \%$ & $\geq 0.05$ & & $86.5 \%$ & $70.3 \%$ & $\geq 0.05$ \\
Specificity & $99.2 \%$ & $95.3 \%$ & $\mathbf{0 . 0 0 0 2}$ & & $99.6 \%$ & $99.6 \%$ & $\geq 0.05$ \\
PPV & $81.0 \%$ & $41.0 \%$ & $\mathbf{0 . 0 0 3}$ & & $94.1 \%$ & $92.9 \%$ & $\geq 0.05$ \\
NPV & $95.0 \%$ & $95.7 \%$ & $\geq 0.05$ & $99.0 \%$ & $97.8 \%$ & $\geq 0.05$ \\
\hline
\end{tabular}

CONCLUSIONS: Enrichment broths were more sensitive and specific for the detection of STX than direct stool samples by the VCA and EIA methods. The Prolisa EIA performed as well or better than the Premier EIA in both direct stool and enriched stool testing. The high positive and negative predictive values of the EIAs indicate they are reasonable frontline screening tools for STX in broth enrichment cultures of stool samples.

\section{J4}

\section{A PROSPECTIVE COMPARATIVE STUDY OF ANTIGEN DETECTION (GALACTOMANNAN) VERSUS CONVENTIONAL DIAGNOSIS OF INVASIVE ASPERGILLOSIS AND IMPACT ON PATIENT OUTCOME}

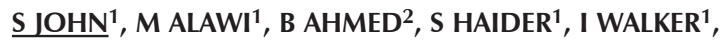
D YAMAMURA ${ }^{1}, \mathrm{C} \mathrm{KIM}^{2}$, C LEE ${ }^{1,2}$

${ }^{1}$ McMaster University; ${ }^{2}$ St Joseph's Healthcare, Hamilton, ON OBJECTIVE: The objective of this study was to compare the test performance of antigen detection (galactomannan) with current diagnostic criteria (clinical manifestations, results of imaging and microbiology) for the diagnosis of invasive aspergillosis (IA). The second objective was to evaluate the impact of antigen testing on the morbidity and mortality of IA in comparison with the existing diagnostic modality.

METHODS: Patients were eligible to participate in this prospective, randomized study if they met criteria for probable/confirmed diagnosis of IA based on US mycology group definitions or were deemed high risk. Patients were recruited from 4 hospitals over a 24 month period. Eligible patients who consented underwent non invasive blood tests.

RESULTS: Of 50 eligible patients, 29 individuals were recruited. $40 \%$ of patients were neutropenic and more than $85 \%$ had a haematological malignancy or had undergone a bone marrow transplant. "Platelia Aspergillus EIA" assay was performed on 69 specimens. 13 samples were positive (8 patients), 2 samples were at cut off value and 54 samples were negative (78\%). In the 8 patients with positive samples, 4 had possible IA based on clinical criteria. None had proven or probable IA. All 8 patients received anti-fungal prophylaxis with fluconazole. 2 of the 8 patients received treatment with voriconazole or ampho B. The mortality rate was 50\% (4/8) and the cause of death for $75 \%$ of cases was fungal pneumonia

CONCLUSION: The galactomannan assay may be a better diagnostic tool than clinical criteria for IA and may also predict mortality in this high risk group.

\section{ROOM: VILLE-MARIE B}

\section{SESSION K}

\section{K1}

\section{POST-ARRIVAL SCREENING FOR MALARIA IN}

ASYMPTOMATIC REFUGEES USING REAL-TIME PCR

C MATISZ ${ }^{1}$, P NAIDU ${ }^{2}$, S SHOKOPLES ${ }^{1}$, D GRICE ${ }^{3}$, V KRINKE $^{3}$, SZ BROWN ${ }^{4}$, K KOWALEWSKA-GROCHOWSKA ${ }^{2}$, S HOUSTON ${ }^{2}$, SK YANOW ${ }^{1,2}$

${ }^{1}$ Provincial Laboratory for Public Health; ${ }^{2}$ University of Alberta; ${ }^{3}$ New Canadians Clinic; ${ }^{4}$ DynaLife Diagnostic Laboratory Services, Edmonton, $\mathrm{AB}$

OBJECTIVE: Malaria is a significant health risk to refugee populations originating from endemic areas yet there is little consensus on screening and/or treatment approaches for malaria in this population. Furthermore, detection of malaria in semi-immune asymptomatic refugees is limited by the sensitivity of diagnostic tests used for screening. We determined the prevalence of malaria by microscopy and real-time PCR in asymptomatic refugees examined in Edmonton, Canada, during 2009-2010.

METHODS: A total of 324 refugees who underwent a medical exam at the New Canadians Clinic in Edmonton between March 2009 and 2010 were included in the study. All were asymptomatic for malaria. Patient demographics, clinical and laboratory data were obtained through review of medical charts and results from electronic laboratory information systems. Routine assessment included a medical history, history of past malaria diagnoses, country of origin and of refugee residence. Blood samples were processed by microscopy immediately following medical screening and served as the standard for patient care. Real-time PCR was performed retrospectively on de-identified samples at the end of the collection year.

RESULTS: The majority of refugees $(97 \%)$ originated in countries that are endemic for malaria. While all thick and thin blood smear results were negative, ten subjects (3.1\%) tested positive for Plasmodium DNA by PCR. Speciation by real-time PCR identified 3 cases of P. falciparum, 5 cases of $P$. vivax, and 1 case of $P$. ovale. One sample could not be speciated. Infections were identified in subjects originating from 3 countries: Democratic Republic of Congo ( 2 P. falciparum and 1 P. ovale), Burma (5 P. vivax) and Liberia (1 P. falciparum). Pre-departure treatment was selfreported in only $11 / 324$ subjects, including three subjects infected with P. falciparum.

CONCLUSION: These results highlight the potential usefulness of realtime PCR in the diagnosis of asymptomatic malaria. Furthermore, our findings suggest that appropriate guidelines for malaria screening should consider the risk of relapsing infections, particularly in refugees arriving from Southeast Asia.

\section{K2}

\section{CHAGAS DONOR SCREENING AT CANADIAN BLOOD SERVICES}

M FEARON $^{1,2}{ }^{1}$ V SCALIA $^{3}$, I DINES ${ }^{1}$, M HUANG $^{1}$, M NDAO $^{4,5}$

${ }^{1}$ Canadian Blood Services; ${ }^{2}$ Department of Pathobiology and Laboratory Medicine, University of Toronto, Toronto; ${ }^{3}$ Canadian Blood Services, Ottawa, ON; ${ }^{4}$ National Reference Centre for Parasitology; ${ }^{5} \mathrm{McGill}$ University Centre for Tropical Diseases, Montreal, QC

OBJECTIVE: Chagas Disease is caused by a protozoan parasite and is transmitted by the bite of a Triatoimine bug indigenous to parts of Latin America. 
Recipients of blood transfusions from infected donors may become infected. In May 2010, Canadian Blood Services (CBS) began screening 'at risk' donors for Chagas Disease. This presentation describes the results of donor testing, follow up and lookback investigations after eight months of testing. METHODS: All donors who answer affirmatively to any of three risk questions (birth or having spent $\geq 6$ months in an endemic area, mother or maternal grandmother born in an endemic area) is tested for Chagas antibody using Abbott PRISM Chagas, a highly automated chemiluminescent assay approved for blood donor screening. Any donor who tests repeat reactive (RR) is confirmed at the National Parasitology Reference Lab at McGill University. Confirmatory testing includes immunoblot and PCR. Samples are also sent to the Quest reference lab used by the U.S. blood suppliers for radioimmunoprecipitation assay (RIPA). Donors are requested to have follow up diagnostic testing for Chagas Disease and are asked to complete a detailed CBS questionnaire. Lookback is performed on every previous donation for every confirmed positive donor.

RESULTS: To date, 8 RR donors have been identified out of 7290 donors tested (approximately $0.1 \%$ of tested donor population), and 7 have confirmed positive. Donors are from Manitoba (3), Ontario (2) and B.C. (2) and range in age from 25 to 63 years. There are 6 males and 1 female. Five donors were born in Paraguay, 1 in Argentina, 1 in Canada (this donor spent time in Paraguay as a missionary). Five donors lived in rural areas as children and 4 recall living in mud or thatched houses. Four had mothers, 3 had grandmothers born in an endemic country. Lookback identified 370 products transfused, of which 45 living recipients could be notified. 24 recipients are known to have tested negative to date.

CONCLUSION: Seven blood donors have been confirmed positive for Chagas Disease. Only one of the donors was aware that he had had Chagas Disease in the past. These donors are now permanently deferred.

\section{K3}

\section{GENOTYPING OF TWENTY YEARS OF PATHOGENIC ACANTHAMOEBA ISOLATES FROM BRITISH COLUMBIA: A PRELIMINARY REPORT}

\section{M-K LEE, S MAN, T LO, Q WONG, J ISAAC-RENTON,} MG MORSHED

British Columbia Centre for Disease Control Public Health Microbiology and Reference Laboratory (BCPHL), Vancouver, BC OBJECTIVE: Acanthamoeba keratitis, a corneal disease caused by members of a genus of free-living amoebae, is predominantly associated with contact lens use. This research aims to genotype Acanthamoeba isolates recovered since 1988 using sequence analysis.

METHODS: Acanthamoeba isolates from patients diagnosed in British Columbia (B.C.) between the years 1988 to 2009 ( $n=45$ ) were subjected to PCR amplification and DNA sequencing targeting a partial sequence of the 18S rRNA gene (ASA.S1, DF3 region). Cycle sequencing was performed with ABI Big Dye ${ }^{\circledR}$ Chemistry and analyzed on the ABI Genetic Analyzer 3130xl. The sequences obtained were analyzed and a phylogenetic tree was built with Geneious software and confirmed with PHYML and MrBayes.

RESULTS: All cultured isolates were confirmed to be Acanthamoeba by PCR. Molecular phylogenetic analysis showed that all the Acanthamoeba isolates studied belonged to the T4 genotype.

CONCLUSION: This study demonstrated the prevalence of the T4 genotype in B.C., which parallels the finding that T4 is the predominant Acanthamoeba keratitis-related genotype worldwide. Some differences between isolates were also demonstrated using this method. This tool may be useful in defining the molecular epidemiology of this parasitic infection.

\section{K4}

MOLECULAR CHARACTERIZATION OF WEST NILE VIRUS STRAINS IN WESTERN NORTH AMERICA

M-K LEE ${ }^{1}$, S MAN ${ }^{1}$, K FERNANDO ${ }^{1}$, T LO ${ }^{1}$, Q WONG ${ }^{1}$, P TANG $^{1}$, B HENRY $^{2}$, V BOWES ${ }^{3}$, S DREWS ${ }^{4}$, R BAKER ${ }^{5}$, MG MORSHED ${ }^{1}$ ${ }^{1}$ British Columbia Centre for Disease Control Public Health Microbiology and Reference Laboratory (BCPHL); ${ }^{2}$ British Columbia Centre for Disease Control Public Health Emergency Services, Vancouver; ${ }^{3}$ Animal Health Centre, Abbotsford, BC; ${ }^{4}$ Provincial Laboratory for Public Health, Calgary, AB; ${ }^{5}$ Oregon State University, Corvallis, OR, United States

OBJECTIVE: We analyzed and compared the sequences of the West Nile virus (WNv) strains detected in British Columbia (BC) and from the neighbouring provinces and states of Alberta (AB), Washington (WA), and Oregon (OR) and 162 sequences obtained from GenBank.

METHODS: PCR positive WNv samples from mosquito pools $(\mathrm{BC}=10$, $\mathrm{AB}=6, \mathrm{WA}=3$, and $\mathrm{OR}=2)$ and birds $(\mathrm{BC}=5)$ were subjected to sequencing of the pre-membrane protein $\mathrm{M}$, membrane protein $\mathrm{M}$, and envelope glycoprotein E regions. Cycle sequencing was performed on the ABI Genetic Analyzer 3130xl. The sequences were assembled and analyzed with Geneious and phylogenetic tree were constructed with PhyML and MrBayes.

RESULTS: Phylogenetic comparisons showed that all isolates from the Pacific Northwest were similar to each other and belonged to Lineage 1 Clade 1a. However, the BC WNv sequences (both 2009 mosquitoes and 2010 birds) were novel and comprised a new subgroup. The closest subgroup to the BC strains was the subgroup formed by two 2007 Alberta isolates, while samples from WA and OR clustered in a separate subgroup. CONCLUSION: All BC WNv isolates originated from the southern interior region of $\mathrm{BC}$ (Okanagan region), and had closely related sequences. Phylogenetic analysis of available viral sequences from $\mathrm{AB}$, WA and $\mathrm{OR}$ suggests that $\mathrm{WNv}$ may have reached $\mathrm{BC}$ through $\mathrm{AB}$. As viral sequences from certain regions may be underrepresented, additional efforts to sequence additional viruses from WA and OR will be useful.

\section{ROOM: ST-ANTOINE B}

\section{SESSION L}

\section{L1}

\section{SEASONALITY OF INFLUENZA-ATTRIBUTABLE MENINGOCOCCAL DISEASE IN CENTRAL ONTARIO, CANADA: IMPLICATIONS FOR TARGETING OF INFLUENZA VACCINATION PROGRAMS}

\section{A TUITE, D FISMAN}

Dalla Lana School of Public Health, University of Toronto, Toronto, ON

BACKGROUND: Invasive meningococcal disease (meningococcal bacteremia and meningitis, IMD) is an important source of morbidity and mortality in children and adolescents in North America. Seasonality of IMD is aligned with that of influenza, and we have identified a significant surge in IMD risk with elevated influenza incidence in central Ontario, Canada in a recent publication (RR per 100 unit increase in influenza case activity $=1.18,95 \%$ CI 1.06 to 1.31 ). We used population-attributable risk percent (PAR\%) calculations to evaluate the implications of influenza control for IMD risk reduction.

METHODS: PAR\% reflects risk among the exposed and population prevalence of exposure. With multiple exposure levels PAR $\%=\sum \mathrm{p}_{\mathrm{i}}\left(\mathrm{RR}_{\mathrm{i}}-1\right) /$ $\left(1+\sum \mathrm{p}_{\mathrm{i}}\left(\mathrm{RR}_{\mathrm{i}}-1\right)\right) ; \mathrm{p}_{\mathrm{i}}$ represents the proportion of the population with the $i^{\text {th }}$ level of exposure and $R_{i}$ represents the relative risk associated with the $\mathrm{i}^{\text {th }}$ level of exposure. We evaluated PAR\% estimates for 6 influenza seasons and 6 non-influenza seasons in Ontario from 2000 to 2006 using FluWatch surveillance data and laboratory surveillance data on IMD.

RESULTS: PAR\% for invasive meningococcal disease within influenza seasons was high, ranging from $66 \%(29 \%-79 \%$ ) (in 2003-2003) to $97 \%$ (56\% to 100\%) (in 2003-2004); in non-influenza seasons PAR\% ranged 
from $26 \%$ (9\% to $36 \%$ ) in 2000 to $60 \%(22 \%$ to $77 \%$ ) in 2003 . Based on weekly estimates of PAR\% 48 of 88 IMD cases (54\%) occurring from 2000 2006 were influenza-attributable.

CONCLUSIONS: Studies supporting a causal role for influenza A in the genesis of seasonal changes in meningococcal disease have important implications for the control of both diseases. Immunization of young age groups against influenza likely represents an under-recognized strategy for preventing IMD in high-risk age groups.

\section{L2}

GOOD COVERAGE OF INVASIVE STREPTOCOCCUS PNEUMONIAE EXPECTED FOR THE 13-VALENT PNEUMOCOCCAL CONJUGATE VACCINE (PCV-13) IN CANADA

HJ ADAM ${ }^{1,2}$, M DeCORBY $^{2}, \mathrm{NM} \mathrm{LAING}^{2}, \mathrm{KA} \mathrm{NICHOL}^{1}$, AK WIERZBOWSKI ${ }^{2}$, I MARTIN ${ }^{3}$, M GILMOUR $^{1,3}$, W DEMCZUK ${ }^{3}$, DJ HOBAN ${ }^{1,2}$, GG ZHANEL ${ }^{2}$

${ }^{1}$ Diagnostic Services of Manitoba; ${ }^{2}$ University of Manitoba;

${ }^{3}$ National Microbiology Laboratory - Public Health Agency of Canada, Winnipeg, MB

OBJECTIVE: The Baseline Epidemiology of Streptococcus pneumoniae (SPN) Serotypes (BESST) study assessed the circulating serotypes of SPN from all age groups in Canada from 2007 to 2009, inclusive, prior to the introduction PCV-13.

METHODS: 399 SPN obtained from blood cultures as part of CANWARD (an annual, ongoing, Canadian surveillance study) were serotyped by the Quellung method and susceptibility testing was performed with CLSI methodology.

RESULTS: Forty-two different serotypes were observed; most commonly, 19A (12\%), 5 (8\%), $3(8 \%), 22 \mathrm{~F}(7 \%)$, and $4(6 \%)$. Overall, $54 \% / 19 \%$ of the strains belonged to PCV-13/PCV-7 serotypes and $9 \% / 23 \%$ to vaccinerelated serotypes, respectively. By age group $(\geq 2,3-16,17-49$, and $\geq 50$ years), $56,71,52$ and $51 \%$ of the strains belonged to PCV- 13 serotypes and $5,5,8$, and $11 \%$, to vaccine-related serotypes, respectively. Selected antimicrobial susceptibilities of PCV-13 vaccine serotypes (VS), PCV-13 vaccine-related serotypes (VRS) and PCV-13 non-vaccine (NV) serotypes are as follows: penicillin [meningitis (M)/nonmeningitis (NM)], 79\%/99\% (VS), 83\%/100\% (VRS), 96\%/100\% (NV); ceftriaxone (M/NM), 99\%/100\% (VS), 100\%/100\% (VRS \& NV), clarithromycin, 86\% (VS), 94\% (VRS), 87\% (NV); and levofloxacin, 100\% (VS, VRS \& NV).

CONCLUSIONS: The current epidemiology of SPN serotypes in Canada demonstrates that PCV-13 will provide good coverage of invasive strains across all age groups. PCV-13 provides significantly more coverage of the currently circulating SPN strains in Canada as compared to PCV-7, including the emerging, multi-drug resistant 19A.

\section{L3}

\section{RISK OF INVASIVE HAEMOPHILUS INFLUENZAE TYPE B (HIB) DISEASE AND RESPONSE TO HIB VACCINE IN PATIENTS WITH CHRONIC RENAL FAILURE}

M ULANOVA $^{1}$, N HAWDON ${ }^{1}$, E NIX ${ }^{1}$, G FERRONI ${ }^{2}$, W MCCREADY ${ }^{1}$ ${ }^{1}$ Northern Ontario School of Medicine, Thunder Bay; ${ }^{2}$ Northern Ontario School of Medicine, Sudbury, ON

OBJECTIVE: Patients with end-stage renal disease undergoing peritoneal or hemodialysis are immunocompromised and therefore at high risk for septicemia and other severe infections. The pediatric conjugate vaccine against Haemophilus influenzae type b (Hib) was introduced in Canada in 1991; the population born before was not immunized. In non-vaccinated adults, protection against Hib is mediated by natural anti-capsular polysaccharide antibodies. Immunocompromised individuals may be at risk of invasive Hib disease because the bacteria still circulate in the population. We hypothesized that adult patients with chronic renal failure (CRF) lack protective antibodies against $\mathrm{Hib}$, but may respond to the pediatric Hib vaccine.

METHODS: Serum anti-Hib IgG levels were studied in 60 adult patients with CRF and 38 healthy controls of similar age using standard ELISA. Thirty-two CRF patients (median age: 65 years) were immunized with one dose of Act-HIB, which contains 10 microgram of Hib capsular polysaccharide conjugated to tetanus toxoid; serum antibody levels were assessed before and 4 weeks after vaccination. Functional antibody activity was studied using a serum bactericidal test.

RESULTS: Almost $90 \%$ of controls, but only $43 \%$ of non-vaccinated CRF patients had protective anti-Hib antibody levels ( $>1.0$ microgram $/ \mathrm{ml})$. Geometrical mean concentrations (GMC) and 95\% confidence intervals in controls were 1.07 (0.41-2.29) versus 0.13 (0.04-0.41) in CRF patients. Following vaccination, all but one patient ( $97 \%$ ) have developed protective antibody levels with a 14-fold increase in GMC $(\mathrm{P}<0.05)$.

CONCLUSION: The majority of adult patients with CRF are at an increased risk of acquiring invasive Hib disease because they lack protective anti-capsular polysaccharide antibodies. The pediatric Hib conjugate vaccine is highly immunogenic in these patients. Considering that $\mathrm{Hib}$ continues to circulate in Canada, this study provides rationale for the immunization of adult immunocompromised individuals with the pediatric Hib vaccine to achieve protective immunity.

\section{L4}

\section{SPATIAL AND TEMPORAL DISTRIBUTION OF TUBERCULOSIS IN THE NORTHWEST TERRITORIES, CANADA}

$\underline{\text { LCHUI }}{ }^{1,2}$, H LI $^{1}, \mathrm{C} \mathrm{CASE}^{3}, \mathrm{~V} \mathrm{LI}^{1}$

${ }^{1}$ Provincial Laboratory for Public Health; ${ }^{2}$ Laboratory Medicine and Pathology, Edmonton, AB; ${ }^{3}$ Department of Health and Social Services, Yellowknife, NWT

INTRODUCTION: Geographical Information System (GIS) is a software system used to map and analyze geographically referenced information. This study uses GIS to analyze the spatial and temporal distribution of tuberculosis (TB) in the Northwest Territories (NWT), based on Mycobacterium tuberculosis fingerprinting patterns in an effort to identify potential transmission trends, at-risk populations and communities, and fingerprint pattern prevalence.

METHODS: Demographic data - including gender, age, ethnicity, date of diagnosis, and patient address - collected between 1990 and 2009 were mapped using ArcGIS software. Space-time permutation scan statistic in SatScan was used to analyze spatial and temporal patterns of DNA fingerprint clusters. Fingerprinting data generated by IS6110-RFLP (Restriction Fragment Length Polymorphism) were clustered using BioNumerics software with the following parameters: UPGMA clustering method, the Dice coefficient, and $1 \%$ position tolerance. Annual spatial distribution for each cluster was mapped to present the spatial pattern and transmitting trend. Meanwhile, thematic maps were generated based on demographic data.

RESULTS: 195 cases of TB in the NWT were grouped into 5 clusters based on DNA fingerprint patterns using BioNumerics software. Our data show that most of these cases were concentrated around the Great Slave Lake and Mackenzie Delta, areas with high population density and established transportation routes. A pattern of TB transmission was also revealed: transmission in the NWT generally occurs from the south to the north, as well as from central cities to the surrounding towns.

CONCLUSION: Potential transmission occurrences identified in this study include working relationships (cluster 3), a nomadic lifestyle (cluster 4), and social networking (cluster 5). Transmission of TB in northernmost areas is most likely due to close networking and contact within the communities. Trends identified in this study suggest TB transmission is largely occurring from southern to northern NWT. However, southern communities, especially Behchoko and Yellowknife, are still at a higher risk in the coming years. 


\section{POSTER PRESENTATIONS THURSDAY, APRIL 7 AND FRIDAY, APRIL 8 ROOM: PALAIS ROOM}

\section{P1}

\section{IMPACT OF 2009 CAPITAL HEALTH VANCOMYCIN MONITORING AND DOSING GUIDELINES ON CLINICAL PRACTICE}

T ELTON $^{1}$, M GRAY $^{1}$, L SAXINGER ${ }^{2}$, P MAYO ${ }^{1}$, S KOSHMAN ${ }^{1,2}$ ${ }^{1}$ Alberta Health Services; ${ }^{2}$ University of Alberta, Edmonton, $A B$ OBJECTIVES: A 2005 Residency project by Lehmann \& Gray established that time to target trough levels of $15-20 \mathrm{mg} / \mathrm{L}$ was delayed when dosing vancomycin based upon ideal body weight (IBW) for most adults. As of February 2009, Capital Health adjusted guidelines to initially dose all patients according to actual body weight (ABW) with a maximum of $2 \mathrm{~g} /$ dose. We sought to determine whether new guidelines were being adhered to by practitioners and assess their impact.

METHODS: We performed a retrospective chart review for individuals at least 18 years of age receiving intravenous vancomycin therapy for at least 2 weeks at the University of Alberta Hospital between February $1^{\text {st }}$ and December $31^{\text {st }}$ of 2009 . We gathered demographic information, initial dosing data (type of infection, dose amount, interval and start time), and trough level data (target and actual levels).

RESULTS: We screened 101 patients (51 targeting 10-15 mg/L, 50 targeting $15-20 \mathrm{mg} / \mathrm{L}$ ). Only $17 \%$ of initial patient doses matched 2009 guideline estimates, of which the average initial trough level for these patients was $10.7 \mathrm{mg} / \mathrm{L}$. Time to reach goal troughs was 7.1 days for targets of $15-20 \mathrm{mg} / \mathrm{L}$ as compared to 3.8 days for targets of $10-15 \mathrm{mg} / \mathrm{L}$.

CONCLUSIONS: We found that clinicians are not adhering to the new guidelines. Furthermore, the new guidelines do not adequately dose to achieve $1^{\text {st }}$ target levels for indications requiring $15-20 \mathrm{mg} / \mathrm{L}$. New calculations are proposed to target either $10-15 \mathrm{mg} / \mathrm{L}$ or $15-20 \mathrm{mg} / \mathrm{L}$.

\section{P2}

A SINGLE DAY PREVALENCE SURVEY OF ANTIMICROBIAL UTILIZATION WITHIN SELECTED CANADIAN ACUTE CARE HOSPITALS: 11\% INCREASE IN USE BETWEEN 2002-2009 D GRAVEL ${ }^{1}$, G TAYLOR ${ }^{2}$, A MATLOW ${ }^{3}$, J EMBIL ${ }^{4}$, E HENDERSON ${ }^{5}$, M JOHN $^{6}$, L JOHNSTON ${ }^{7}$, N LE SAUX ${ }^{8}$, J EMBREE ${ }^{4}$, V ROTH ${ }^{9}$, A WONG ${ }^{10}$, L SAXINGER ${ }^{2}$, K WEISS ${ }^{11}$, Canadian Nosocomial Infection Surveillance Program ${ }^{1}$

${ }^{1}$ Centre for Communicable Diseases and Infection Control, Public Health Agency of Canada, Ottawa, ON; ${ }^{2}$ University of Alberta Hospital, Edmonton, AB; ${ }^{3}$ The Hospital for Sick Children, Toronto, ON; ${ }^{4}$ Health Sciences Centre, Winnipeg, MB; ${ }^{5}$ Calgary Health Region, Calgary, $\mathrm{AB}$; ${ }^{6}$ London Health Sciences Centre, London, $\mathrm{ON}$; ${ }^{7}$ Queen Elizabeth II Health Sciences Centre, Halifax, NS; ${ }^{8}$ Children's Hospital of Eastern Ontario; ${ }^{9}$ The Ottawa Hospital, Ottawa, ON; ${ }^{10}$ Royal University Hospital, Sasktoon, SK;

${ }^{11}$ Maisonneuve-Rosemont Hospital, Montreal, QC

OBJECTIVES: To determine the prevalence, and describe patterns of antimicrobial use in Canadian acute care hospitals participating in the Canadian Nosocomial Infection Surveillance Program during a single day prevalence survey of healthcare-associated infections, in follow up of an identical 2002 survey.

METHODS: A point-prevalence survey of healthcare associated infections was conducted on February 3, 2009 in 49 hospitals across Canada. Information including antimicrobial use was collected on all hospitalized inpatients.

RESULTS: 9953 patients were surveyed; 8599 (86.4\%) were adults ( $\geq 18$ years of age), $622(6.4 \%)$ were 1 to 17 years of age, and $732(7.4 \%)$ were infants under the age of 1 years. Overall, $42.7 \%$ of patients were on Medicine or Pediatric Units, with $30.1 \%$ on Surgery and $8.9 \%$ on Critical or Intensive Care units. 3998 (40.2\%) patients were on one or more antimicrobial agents on the day of the survey $(3434,40.1 \%$ adults, $329,52.9 \%$ children; and $213,29.4 \%$ of the infants). The most commonly prescribed antimicrobial class in adults was fluoroquinolones (1023, 29.8\%); followed by cephalosporins $(925,26.9 \%)$ penicillins $(784,22.8 \%)$ and vancomycin $(475,13.8 \%)$. Children and infants were more frequently prescribed penicillin class antimicrobial $(204,31.6 \%$ and $105,49.3 \%$ respectively), followed by aminoglycosides $(65,19.8 \%$ and $73,34.3 \%)$. Patterns of use in different hospital settings will be presented.

CONCLUSIONS: Compared to our previous point prevalence survey conducted in 2002, there was an $11 \%$ increase in antimicrobial utilization from $36.2 \%$ to $40.2 \%$ ( $p<0.0001$ ). With the increasing importance of antimicrobial resistance, these results may help derive an approach to benchmarking antimicrobial use in various different hospital settings and guide antimicrobial stewardship programs.

\section{P3}

DEVELOPING AN ANTIFUNGAL PROPHYLAXIS ALGORITHM IN HIGH RISK NEUTROPENIC PATIENTS USING SURVEILLANCE OF LOCAL INVASIVE FUNGAL DISEASE PREVALENCE AND MODELLING BASED ON PUBLISHED DATA

\section{SAXINGER $^{1}$, S FRYTERS ${ }^{2}$, K DOUCETTE $^{1}$}

${ }^{1}$ University of Alberta; ${ }^{2}$ Alberta Health Services, Edmonton, $A B$ BACKGROUND: Studies suggest that antifungal prophylaxis can decrease incidence of invasive fungal disease (IFD) in high risk neutropenic patients, but the impact of prophylaxis depends on the local prevalence of IFD. Stewardship of these agents should include a cost analysis of prophylaxis, as a variety of approaches are feasible. Prospective surveillance of IFD in high risk neutropenic patients (ANC of $0.5 \times 10^{9} / \mathrm{L}$ or less, for at least 10 days) has been carried out at our centre since January 1 , 2009, providing data for this analysis.

METHODS: Using our surveillance data and published efficacy data, we estimated the efficacy and drug cost for posaconazole prophylaxis versus no prophylaxis in the highest risk subset of our patient population. In addition, published Canadian data was used for a cost analysis of IFD related hospitalization.

RESULTS: Assuming 40 induction/reinduction AML patients per year, the total cost (to nearest $1 \mathrm{~K}$, including all antifungal drug management costs) to prevent one IFI is $\$ 14 \mathrm{~K}$, one all cause death $\$ 6 \mathrm{~K}$, with an overall incremental antifungal cost of $\$ 42 \mathrm{~K}$ in using prophylaxis. Incorporating IFD management costs suggested an overall savings of $\$ 52 \mathrm{~K}$. Our proposed algorithm will be presented.

\begin{tabular}{lcccrr} 
Condition & $\begin{array}{c}\text { Surveillance } \\
\text { Data 2009 }\end{array}$ & $\begin{array}{c}\text { Estimated change } \\
\text { with Posaconazole }\end{array}$ & $\begin{array}{c}\text { RR } \\
\text { Reduction }\end{array}$ & $\begin{array}{c}\text { AR } \\
\text { Reduction }\end{array}$ & NNT \\
\hline All IFI & $10.10 \%$ & $2.77 \%$ & $72.6 \%$ & $7.33 \%$ & 14 \\
Aspergillosis & $4.00 \%$ & $0.39 \%$ & $90.2 \%$ & $3.61 \%$ & 28 \\
All cause Mortality & $56.00 \%$ & $37.33 \%$ & $33.3 \%$ & $18.67 \%$ & 5 \\
Attributable Mortality & $22.00 \%$ & $14.67 \%$ & $33.3 \%$ & $7.33 \%$ & 14 \\
\hline
\end{tabular}

CONCLUSION: In this population, the drug cost and NNT of antifungal prophylaxis with posaconazole is in keeping with other interventions, and may be cost saving.

\section{P4}

COMPARISON OF MORTALITY BETWEEN NOSOCOMIAL AND COMMUNITY ACQUIRED FEBRILE NEUTROPENIA PATIENTS TREATED INITIALLY WITH CEFAZOLIN AND TOBRAMYCIN: RETROSPECTIVE CHART REVIEW M ELLIGSEN $^{1}$, SAN WALKER ${ }^{1,2}$, SE WALKER ${ }^{1,2}$, F LEPIANE $^{3}$, N LATHIA $^{1,2}$, C De'ANGELIS $^{1,2}$, A SIMOR $^{1,2}$

${ }^{1}$ Sunnybrook Health Sciences Centre, North York; ${ }^{2}$ University of Toronto; ${ }^{3}$ Univeristy Health Network, Toronto, ON

OBJECTIVE: Cefazolin and tobramycin is the recommended empiric regimen for febrile neutropenia (FN) at our institution. This regimen was determined to be effective for community acquired FN but had not been evaluated in nosocomial FN. The objectives of this study were to compare all cause mortality from 2002-04 with 2008-09 in nosocomial FN 
empirically treated with cefazolin and tobramycin and to compare the characteristics of patients with nosocomially acquired FN to community acquired FN.

METHODS: A retrospective chart review of 45 nosocomially acquired FN episodes from 2008-09, and 50 episodes before Dec 31/04 empirically treated with cefazolin and tobramycin was conducted. Data on the community acquired FN episodes were obtained from our earlier research.

RESULTS: All cause mortality in nosocomially acquired FN increased from $4 \%$ in $2002-04$ to $13 \%$ in 2008-09 ( $\mathrm{p}=0.14$ ). The nosocomial cohort was at higher risk of medical complications and mortality than the community acquired cohort based on several variables (neutrophil nadir, duration of neutropenia and fever, hematological malignancy, MASCC and Talcott score $)(\mathrm{p}<0.05)$. As a result, patients in the nosocomial cohort were treated with longer courses of antibiotic therapy and were more likely to require broader spectrum antibiotic coverage $(\mathrm{p}<0.05)$.

CONCLUSIONS: A signal of increased risk of mortality from 2002-04 compared to 2008-09 was observed in patients empirically treated with cefazolin and tobramycin for nosocomial FN. Therefore, this regimen is not appropriate for nosocomial FN.

\section{P5}

\section{BORDATELLA HOLMESII BACTEREMIA IN NORTHERN ALBERTA: A 5-YEAR CASE REVIEW}

LKANII ${ }^{1}$, S GEE ${ }^{2}$, J AHMED-BENTLEY ${ }^{3}$, M-C LEE $^{3}$, J NIGRIN ${ }^{3}$, R VERITY $^{3}$, N SOLOMON ${ }^{1,2}$

${ }^{1}$ University of Alberta; ${ }^{2}$ Provincial Laboratory for Public Health (Microbiology); ${ }^{3}$ DYNALife Dx Microbiology Laboratory, Edmonton, AB

BACKGROUND/OBJECTIVE: Bordetella holmesii is an emerging pathogen that can cause invasive disease in immunocompromised (particularly asplenic) patients. Due to the fastidious growth requirements of this organism, it can be difficult to identify in the routine microbiology laboratory. The aim of this study was to carry out a 5 -year review of B. holmesii cases in Northern Alberta.

METHODS: Cases of B. holmesii bacteremia were identified retrospectively at the Provincial Laboratory for Public Health (Microbiology) at the University of Alberta Hospital. Blood culture isolates referred for identification to the Provincial Laboratory by DYNALife Microbiology Laboratories during the pre-identified 5-year period were also included in our review.

RESULTS: From October 2005 - November 2010, 8 cases of B. holmesii bacteremia were identified. $50 \%$ were from the Edmonton region and one case was from the Northwest Territories. The average age was 43 years (range 4 weeks - 66 years) and $62.5 \%$ were male. Identified comorbidities included ulcerative colitis on prednisone ( 2 patients), asplenia, common variable immune deficiency, and type 2 diabetes mellitus. Cough, fever, and upper respiratory tract symptoms were the presenting manifestations in $50 \%$ of patients. The organism usually grows poorly on blood-agar plates, but the growth can be enhanced by incubating plates at $80-90 \%$ humidity for 24-48 hours. Identification was confirmed using biochemical testing, cellular fatty acid analysis, and confirmation by sequencing at the National Microbiology Laboratory (Winnipeg, Manitoba). The most effective antibiotics were found to be ciprofloxacin, levofloxacin, piperacillin-tazobactam, meropenem, and imipenem. None of the patients in this review died from B. holmesii infection.

CONCLUSIONS: B. holmesii can cause infections in both healthy and immunocompromised individuals. Although it is an organism of low virulence and susceptible to many common antimicrobials, infection with B. holmesii can cause severe sepsis. The organism can be difficult to initially identify if not grown in high humidity which may delay identification.

\section{P6}

PROSTHETIC JOINT INFECTION CAUSED BY BORDETELLA HOLMESII

S ABOUANASER ${ }^{1}$, J SRIGLEY ${ }^{1}$, L WILCOX ${ }^{1}$, J JOHNSTONE ${ }^{2,3}$

${ }^{1}$ Department of Pathology and Molecular Medicine; ${ }^{2}$ Department of Medicine, McMaster University; ${ }^{3}$ Michael G DeGroote Institute for Infectious Disease Research, Hamilton, ON

OBJECTIVES: To describe the first reported case of prosthetic joint infection caused by B. holmesii and to review the features of this organism.

METHODS: Case report and review of the literature.

RESULTS: A 54-year-old immunocompetent woman presented to hospital with a suspected right prosthetic knee infection following bilateral knee replacement in 2008. The patient was started empirically on IV cefazolin and the prosthesis was removed. Cultures from both the knee aspirate and intraoperative tissue grew a fastidious Gram-negative bacillus (GNB) after 5 days of incubation, which could not be identified by biochemical analysis. Cellular fatty acid analysis identified the organism as B. holmesii, and this identification was confirmed using matrix-assisted laser desorption/ ionization time-of-flight mass spectrometry. The most common clinical manifestation of $B$. holmesii infection is bacteremia in asplenic patients, but it has also been reported to cause endocarditis, pneumonia, cellulitis, and pyelonephritis, usually in immunocompromised patients. There are only 2 reported cases of septic arthritis, 1 in an immunocompromised patient and 1 in a healthy adolescent. B. holmesii is generally susceptible in vitro to fluoroquinolones, trimethoprim-sulfamethoxazole and carbapenems, but clinical experience is limited and the optimal treatment regimen is uncertain.

CONCLUSION: B. holmesii is a fastidious GNB that is associated with bactremia and a variety of other infections, usually in asplenic and otherwise immunocompromised patients. This is the first reported case of prosthetic joint infection caused by B. holmesii.

\section{ROOM: VIGER}

\section{P7}

\section{DESULFOVIBRIO SPP BACTEREMIA ASSOCIATED WITH UNDERLYING GASTROINTESTINAL ILLNESSES} N MINA ${ }^{1}$, F SHING ${ }^{2}$, P KIBSEY ${ }^{3,4}$, D PURYCH ${ }^{4,5}$, T RAHIM ${ }^{2}$, D ROSCOE 4,6, L HOANG ${ }^{2,4}$

${ }^{1}$ University of Alberta, Edmonton, AB; ${ }^{2}$ BCCDC Public Health and Reference Microbiology Laboratory, PHSA, Vancouver; ${ }^{3}$ Royal Jubilee Hospital, Victoria; ${ }^{4}$ UBC Department of Pathology and Laboratory Medicine, Vancouver ${ }^{5}$ Royal Columbian Hospital, New Westminster ${ }^{6}$ Vancouver General Hospital, Vancouver, BC OBJECTIVE: We report on a series of 5 Desulfovibrio bacteremia cases associated with gastrointestinal (GI) diseases.

METHOD: Isolates from blood culture sources submitted to a provincial reference laboratory between 2008 and 2010 were evaluated by phenotypic and $16 \mathrm{~S}$ rRNA sequencing in parallel. A number of Desulfovibrio spp were unidentifiable by commercial identification systems and reference level conventional phenotypic methods. Identification required sequencing. Detailed clinical information was sought by retrospective clinical and laboratory chart review.

RESULTS: Four slow growing, gram negative anaerobic bacilli were identified as Desulfovibrio desulfuricans and one as D. intestinalis using $16 \mathrm{~S}$ rRNA sequencing during the period of study. Patients' ages ranged from 61-86 yo with a male to female ratio of 3:2. All patients presented with or had a recent history of underlying GI diseases including diverticular disease and choledocholithiasis. Two of 5 cases had pure blood cultures with Desulfovibrio sp, while the rest had polymicrobial bacteremia.

CONCLUSION: Our study demonstrated the first case series linking Desulfovibrio spp to bacteremia in elderly patients with underlying GI diseases. This is also the first reported clinical case in the literature of $D$. intestinalis causing human disease. Difficulties in phenotypically identifying Desulfovibrio isolates were resolved by $16 \mathrm{~S}$ sequencing. Identification of Desulfovibrio spp at least on the genus level helps to identify the source of infection, likely gastrointestinal or biliary tree. 
P8

ACTINOMYCETE BACTEREMIA CAUSED BY SACCHAROPOLYSPORA SP

C TURENNE $^{1}$, J DELPORT ${ }^{2}$, T BURDZ ${ }^{3}$, D WIEBE ${ }^{3}$, D THIRY $^{1}$, N ANTONISHYN ${ }^{1}$, E NAGLE ${ }^{1}$, PN LEVETT ${ }^{1}$, GB HORSMAN ${ }^{1}$, K BERNARD $^{3}$

${ }^{1}$ Saskatchewan Disease Control Laboratory; ${ }^{2}$ Regina General Hospital, Regina, SK; ${ }^{3}$ National Microbiology Laboratory, Public Agency of Canada, Winnipeg, MB

OBJECTIVE: A 58 y. old male presented with febrile episodes of unknown origin. His blood culture grew long branching Gram positive bacilli and he was referred for infective endocarditis follow-up.

METHODS: Identification of the isolate (NML090439) was attempted by API, biochemical tests, FAME analysis and 16 S sequencing. Antimicrobial susceptibility testing was performed by broth microdilution.

RESULTS: The API Coryne profile did not reveal a valid identification. Phylogenetic analysis of the $16 \mathrm{~S}$ gene (1476 bp) revealed a $99.3 \%$ and 98.8\% sequence identity with Saccharopolyspora gloriosae and S. gregorii, respectively. However, the only existing type strain sequence of $S$. gregorii was of poor quality, contributing to a lower sequence identity. Re-analysis using only the first 500 bp revealed a 100\% match to a blood culture isolate labeled as S. gregorii, though this information remains unpublished. Phenotypically, the isolate differed by several characteristics in comparison with both S. gloriosae and S. gregorii, which may suggest a novel species. The organism was susceptible to all antibiotics tested using CLSI aerobic actinomycetes breakpoints.

CONCLUSION: We describe a first case of actinomycete bacteremia caused by a species of Saccharopolyspora. The patient was treated with penicillin for 4 weeks, with negative blood cultures after 1 week. Investigations for infective endocarditis were inconclusive and it was assumed the bacteremia was secondary to poor dentition in most of the lower teeth. This case presented with diagnostic challenges associated with poor sequence quality of GenBank 16S sequences and a lack of information in the literature.

\section{P9}

\section{PSEUDALLESCHERIA BOYDII WRIST SEPTIC ARTHRITIS/ OSTEOMYELITIS IN A PATIENT WITH RHEUMATOID ARTHRITIS RECEIVING ETANERCEPT}

\section{KANII, J FULLER, S SMITH, L MIEDZINSKI}

University of Alberta, Edmonton, AB

BACKGROUND: With the growing use of monoclonal antibody therapies to treat various inflammatory and autoimmune conditions, an increasing number of infectious complications are being reported.

CASE: A 52 year old man with rheumatoid arthritis treated with methotrexate, leflunomide, and etanercept presented with acute painful swelling over the right wrist several days after having received an intra-articular triamcinolone injection into his wrist. Ultrasound guided aspiration of the wrist compartments revealed fungal elements on gram stain. On operative exploration, there was noted to be extensive infection of the fourth, fifth, and sixth compartments of the wrist as well as the entire extensor retinaculum. Moderate amounts of purulent material were sent for culture which subsequently grew $P$. boydii. The patient was started on combination therapy with voriconazole and terbinafine for $P$. boydii osteomyelitis of the wrist. Therapy with etanercept was stopped. We questioned whether the infection was introduced into the wrist via the triamcinolone injection. At 1 year post debridement, the patient is doing well but has developed extensive fibrotic tissue in the area of the previous debridement.

DISCUSSION: Pseudallescheri boydii is a ubiquitous saphrophytic fungus that is known to cause infections in both immunocompetent and immunocompromised hosts. To our knowledge, this is the first osteoarticular infection by $P$. boydii complicating the use of etanercept in the treatment of an inflammatory condition. It is important that clinicians be aware of $P$. boydii as a cause of osteomyelitis and septic arthritis in patients receiving therapy with monoclonal antibodies, treatment for which (based on previous case reviews) is a combination of surgical debridement with antifungal therapy for an extended period.

\section{P10}

\section{ANIDULAFUNGIN FOR TREATMENT OF INVASIVE CANDIDIASIS/CANDIDEMIA: A TERTIARY CENTER CANADIAN EXPERIENCE}

\section{S HAIDER, A BROOKS}

McMaster University, Hamilton Health Sciences, Hamilton, ON

OBJECTIVES: Epidemiological studies have highlighted the emergence of Non-Candida albicans sp, while early effective antifungal therapy has been shown to impact on mortality. Echinocandins have emerged as first line therapy for patients with Candidemia with history of prior azole exposure and or hemodynamic instability.

METHODS: All new antiinfectives introduced at our institution are audited for appropriate utilization. Anidulafungin was introduced in 2008, as an alternative echinocandin, to be restricted to Infectious Disease Specialists for treatment of Candidemia/Invasive Candidiasis in patients with hemodynamic instability. A retrospective chart review was done using the pharmacy databases and electronic medical records, for the period up to November 2010.

RESULTS: Anidulafungin was utilized in 18 patients with age range of 25-88yrs. 12/18 patients had Candidemia or Invasive Candidiasis: 8/18 patients had confirmed Candidemia, while 4 patients had Candida sp. cultured from a sterile site. Candida albicans was seen in 13/18 patients, with C. parapsilosis (2), C. glabrata (1), and C. tropicalis (1). Overall mortality was $56 \%$.

CONCLUSIONS: Mortality from Candidemia underscores the need for newer treatment options. Our audit confirms appropriate use of anidulafungin for patients with documented invasive candidiasis and candidemia. Anidulafungin does not require renal or hepatic adjustment and as such is effective for candidemia/IC with evidence of multiorgan failure.

\section{P11}

DETECTION OF SHIGA TOXIN-PRODUCING E COLI (STEC) USING ENZYME-LINKED IMMUNOSORBENT ASSAY AND

\section{REAL-TIME PCR}

$\underline{L C H U I}^{1,2}$, P KWONG $^{1}$

${ }^{1}$ Provincial Laboratory for Public Health; ${ }^{2}$ Laboratory Medicine and Pathology, University of Alberta, Edmonton, AB

INTRODUCTION: Shiga toxin-producing Escherichia coli (STEC) are food and waterborne pathogens that can cause hemorrhagic diarrhea and can lead to hemolytic uremic syndrome. The O157:H7 serotype is associated with most outbreaks; however non-O157 serotypes are also attributed to diarrheal disease. This study identified STEC in stool samples with realtime PCR, compared toxin expression with PCR results by Enzyme-linked Immunosorbent Assays (EIA), and immunochromatographic tests. Finally all STEC were isolated and serotyped.

METHODS: Diarrheal stool samples were collected from hospitals and communities in selected zones in Alberta during the summer of 2010. Stool culture was incubated in MacConkey broth overnight at $37^{\circ} \mathrm{C}$ and DNA was extracted for real-time PCR to detect the presence of Shiga toxin genes $(s t \times 1$ and $s t \times 2)$. Premier ${ }^{\text {TM }}$ EHEC EIA and ImmunoCard STAT! ${ }^{\circledR}$ EHEC commercial kits were used to detect the presence of Shiga toxin in the overnight enriched stool samples. STEC positive colonies were then isolated on selective media and random colonies were picked for PCR confirmation. As well, STEC and single colonies were picked and cultured for subsequent serotyping.

RESULTS: Of 946 stool samples, 10 (1.1\%) were positive for STEC. Three positive samples carried only the $s t x 1$ gene, three carried only the $s t \times 2$ gene, and four carried both genes. Over half of the STEC samples were found to have non-O157 serotypes: O26, O121, O5, O6, O111 and O145. The real-time PCR data correlated with EIA and ImmunoCard STAT! ${ }^{\circledR}$ EHEC results.

CONCLUSIONS: The results show that real-time PCR, EIA, and ImmunoCard STAT! ${ }^{\circledR}$ EHEC tests are effective methods of detecting STEC and there is $100 \%$ concordance between all three assays. Over half of the STEC positives are non-O157, suggesting that these serotypes are underdiagnosed. Current frontline diagnostic laboratories do not use either real-time PCR, EIA, or immunochromatographic tests to screen for STEC. 
This study outlines the importance of including non-O157 STEC detection in medical diagnostics laboratories.

\section{P12}

\section{A REVIEW OF A NEW TWO STEP ALGORITHM TO DETECT TOXIGENIC CLOSTRIDIUM DIFFICILE}

\section{ЦKRAMER, L FEDIRKO, L DAWSON}

Regina Qu'Appelle Health Region, Regina, SK

OBJECTIVE: Clostridium difficile is the leading cause of nosocomial and antibiotic-associated diarrhoea. Prompt and accurate detection of toxigenic strains is imperative to enable early treatment. Studies have shown that immunologic detection of the constitutive antigen glutamate dehydrogenase $(\mathrm{GDH})$ in stool is more sensitive but less specific than detection of toxin A \& B by enzyme immunoassay (EIA). Therefore, in testing for C.difficile toxin (CDT), our laboratory implemented a two step algorithm to detect GDH, CDT and cytotoxin (CT). The objective of this study was to determine CDT positivity rates and turn around time (TAT) of this two step method.

METHODS: 1054 stool samples were received for C.difficile testing 01/06 -31/08, 2010. 117 were formed stools which were not tested. Stool specimens of liquid or unformed consistency were tested the day of receipt or stored at $4^{\circ} \mathrm{C}$ and tested the following day. Batch testing was increased to twice daily. Samples were tested with C. DIFF QUIK CHEK COMPLETE (Techlab, 2009) according to manufacturers' instructions. GDH+/CDT+ tests were reported as positive and $\mathrm{GDH}$-/CDT- tests were reported as negative. GDH+/CDT- tests were reported as C. difficile antigen positive/ toxin negative; specimen sent to reference laboratory for cytotoxin testing (CT). CT results were reported when complete.

RESULTS: $92 \%$ of test requests were finalized within $24 \mathrm{hrs}$ of receipt as GDH-/CDT- (826) or GDH+/CDT+ (34) and the remaining $8 \%$ of tests $\mathrm{GDH}+/ \mathrm{CDT}$ - (77) were reported as Referred Out for CT (CT: CT + = 16, $\mathrm{CT}-=61)$.

CONCLUSION: Positivity rate was $5.3 \%$. Final TAT decreased for $8 \%$ of specimens. Using a two step algorithm increases the sensitivity and specificity of testing for toxigenic C.difficile. Additional review of the positivity rates and TAT will be done.

\section{P13}

EVALUATION OF TECHLAB QUICK CHEK ${ }^{\text {TM }}-60$ GLUTAMATE DEHYDROGENASE TEST FOR DETECTION OF CLOSTRIDIUM DIFFICILE IN CLINICAL SPECIMENS G BROUKHANSKI $^{1,2}$, A MAKI $^{1}$, A PERALTA ${ }^{1}$, DR PILLAI ${ }^{1,2}$ ${ }^{1} \mathrm{OAHPP}$, PHL-Toronto; ${ }^{2} \mathrm{LMP}$, University of Toronto, Toronto, ON OBJECTIVE: Accurate and rapid detection of Clostridium difficile (CD) is essential for the appropriate treatment and isolation of patients to prevent outbreaks of CD. The Techlab Tox A/B II ${ }^{\mathrm{TM}}$ (TTAB) EIA is widely used because of low cost and high throughput but may lack sensitivity. We evaluated the Techlab QUICK CHEK ${ }^{\mathrm{TM}}$-60 (QC-60) EIA for detection of $\mathrm{CD}$ glutamate dehydrogenase $(\mathrm{GDH})$ as a method to screen specimens negative for $\mathrm{CD}$ compared to a molecular assay (BD GeneOhm ${ }^{\mathrm{TM}}$ (BDGO)) and toxigenic culture.

METHODS: 265 specimens (166 negative and 99 positive by TTAB) were tested with QC-60, TTAB, BD GeneOhm ${ }^{\mathrm{TM}}$ (BDGO) and toxigenic culture. For toxigenic culture (gold standard), colonies grown on CDMN plates were identified using gram stain, fluorescence, and biochemicals. DNA was extracted from colonies confirmed as CD and tested for the presence of toxins genes.

RESULTS: 123 specimens were positive and 142 negative by QC-60. Sensitivity and specificity of QC-60 without confirmation was $98 \%$ and $92 \%, \mathrm{BDGO}-87 \%$ and $93 \%, \mathrm{TTAB}-77 \%$ and $97 \%$ at the first testing and $86 \%$ and $97 \%$ at retesting. After confirmation with the BDGO, specificity of QC-60 increased to $98 \%$ whereas after using the TTAB - to $99 \%$.

CONCLUSION: The Techlab QC-60 is more sensitive than Tox A/B $\mathrm{II}^{\mathrm{TM}}$ when used alone but lacks specificity. When used in the two-step format where TTAB and BDGO are confirmatory tests, the specificity of the QC-60 increased significantly and outperformed TTAB and BDGO.

\section{P14}

RAPID AND ACCURATE DIAGNOSIS OF CLOSTRIDIUM DIFFICILE INFECTION

HR DEVLIN ${ }^{1,2}$, C JACKSON ${ }^{1}$, O PANGAN ${ }^{1}$, G BROUKHANSKI ${ }^{2,3}$, DR PILLAI ${ }^{2,3}$

${ }^{1}$ Department of Laboratory Medicine and Keenan Research Centre of the LiKa Shing Knowledge Institute, St Michael's Hospital; ${ }^{2}$ Department of Laboratory Medicine and Pathobiology, University of Toronto; ${ }^{3}$ Ontario Agency for Health Protection and Promotion, Toronto, ON

OBJECTIVE: Enzyme immunoassays for detection of Clostridium difficile (CD) are insensitive. The BD GeneOhm ${ }^{\mathrm{TM}} \mathrm{CD}$ assay is licensed to be carried out on the SmartCycler. Not all laboratories have a SmartCycler. The role of screening using Glutamate Dehydrogenase (GDH) remains controversial. We compared the TOX A/B II ${ }^{\mathrm{TM}}$ from Techlab (EIA), the BD GeneOhm ${ }^{\mathrm{TM}}$ Cdiff assay run on the Roche LightCycler Instrument, GDH detection using QUICK CHEK ${ }^{\mathrm{TM}}-60$ and toxigenic culture.

METHODS: 200 patient samples were tested. All samples were kept at $4^{\circ} \mathrm{C}$ until tested. TOX A/B II ${ }^{\mathrm{TM}}$ and GDH using QUICK CHEK ${ }^{\mathrm{TM}}-60$ were done according to manufacturer's instructions. DNA was extracted, using the BD GeneOhm ${ }^{\mathrm{TM}}$ kit, and run on the Roche LightCycler 2.0. Samples were plated onto CCFA and CDMN after alcohol shock, incubated anaerobically, identified using fluorescence and latex agglutination, and sent to the Public Health Laboratories - Toronto, OAHPP, for toxin testing using multiplex PCR.

RESULTS: 15 patients were EIA toxin positive, 21 patients were PCR positive and 24 patients were GDH positive. 3 patients were EIA, PCR and GDH positive, but negative by toxigenic culture. The sensitivity and specificity respectively were $60 \%$ and $98.3 \%$ for toxin EIA, $90 \%$ and $98.3 \%$ for PCR and $100 \%$ and $97 \%$ for GDH when compared to toxigenic culture. CONCLUSION: The BD GeneOhm test using the LightCycler is more sensitive than Tox A/B II ${ }^{\mathrm{TM}}$. GDH can be used to screen for $\mathrm{CD}$ prior to PCR for toxin genes. While cost effective, there would be a delay in results reporting.

\section{P15}

EVALUATION OF A COMMERCIAL KIT THE ILLUMIGENE DNA AMPLIFICATION ASSAY TO DETECT CLOSTRIDIUM DIFFICILE AGAINST IN-HOUSE DEVELOPED RT-PCR METHOD

$\underline{\text { SOHN }}^{1,2}$, L MONKMAN ${ }^{1}$, C LEE $^{1,2}$, P JAYARATNE $^{1,2}$

${ }^{1}$ Hamilton Regional Laboratory Medicine Program - Hamilton

Health Sciences and St Joseph's Healthcare; ${ }^{2}$ McMaster University, Hamilton, ON

OBJECTIVES: Illumigene kit is a rapid method for identification of Clostridium difficile based on DNA amplification assay. This study assessed the accuracy of Illumigene detecting Clostridium difficile from freshly collected stools against the in-house RT-PCR method.

METHODS: Stools for Clostridium difficile testing using the in-house developed RT-PCR were tested using the Illumigene method. Consecutive positive and negative specimens collected December 2010 were tested. The stools were maintained at $-20^{\circ} \mathrm{C}$. They were then thawed at room temperature and tested within the hour using the Illumigene kit and determined using the Meridan illumipro-1 Incubator/Reader. Observations were noted on the stools' initial consistency and colour. Results were compared to in-house RT-PCR method (EasyMAG extraction and Rotorgene analysis).

RESULTS: The stools tested ( $\mathrm{n}=76)$ were negative for Clostridium difficile $(\mathrm{n}=43)$, and positive for Clostridium difficile $(\mathrm{n}=33)$ by the in-house method. The Illumigene kit detected $32 / 33$ positives, and 42/43 negatives. There were 3 invalid tests. The in-house PCR method called negative 3 positives that were detected by the Illumigene kit. The in-house method underwent modification and the 3 negatives were re-tested as positive. The Illumigene test procedure took 1 hour including extraction time requiring one piece of equipment. The in-house RT-PCR method took 4 hours including prep time, extraction time (on EasyMAG) and analysis time (on Rotorgene). 
CONCLUSIONS: The Illumigene method is a rapid and accurate method for identification of Clostridium difficile from stool specimens.

\section{P16 \\ COMPARISON OF TEST PERFORMANCE CHARACTERISTICS OF AN IN-HOUSE REAL-TIME MULTIPLEX PCR METHOD WITH COMMERCIAL PCR ASSAYS FOR DETECTION OF TOXIGENIC C. DIFFICILE FROM ADULT STOOLS} P JAYARATNE ${ }^{1,2}$, L MONKMAN ${ }^{1}$, B AHMED ${ }^{1}$, G BROUKHANSKI ${ }^{3,4}$, DR PILLAI $^{3,4}$, C LEE $^{1,2}$

${ }^{1}$ Hamilton Regional Laboratory Medicine Program - Hamilton Health Sciences and St Joseph's Healthcare; ${ }^{2}$ Department of Pathology and Molecular Medicine, McMaster University, Hamilton; ${ }^{3}$ Department of Laboratory Medicine \& Pathobiology, University of Toronto; ${ }^{4}$ Ontario Agency of Public Health Protection and Promotion, Toronto, ON

OBJECTIVES: The objective of the present study was to compare the performance of an in-house real-time PCR method to 3 commercially available PCR assays used to detect toxigenic C. difficile from stools. METHODS: The in-house PCR amplified and detected a fragment of $t c d C, c d t A$ and a eubacterial ribosomal gene target (internal control). Genes were amplified and detected using the Qiagen QuantiTect ${ }^{\mathrm{TM}}$ Mutiplex PCR kit supplemented with respective primers and Taqman Probes in a Rotorgene 6500. DNA from unformed stools from 470 specimens from adults suspected of infection was tested. Three commercial Real-Time PCR assays - proGastro $\mathrm{Cd}^{\circledR}$ (Gen-Probe), BD GeneOhm (Becton Dickinson) for the detection of toxin B gene, and Astra Clostridium difficile PCR Kit 1.0 (Astra Diagnostics) for the detection of toxin A and/or B gene - were used for comparison. All discordant results were tested by culture and toxin assay. The expanded gold standard was defined as $100 \%$ concordance between tests and for discrepant results, positive culture showing toxigenic strains.

RESULTS: Forty one of 470 specimens were identified to have toxigenic C. difficile using the expanded gold standard. Fourteen of 41 toxigenic specimens showed discrepant results by at least one genotypic method. The test performance characteristics showed PCR in-house method was comparable to all commercial assays tested. However, the in-house method is about $30 \%$ cheaper than the least expensive commercial PCR assay. CONCLUSIONS: The in-house PCR used here is a highly sensitive, costeffective method to detect toxigenic C. difficile from adult stools.

\section{P17}

PREVENTION OF CLOSTRIDIUM DIFFICILE INFECTIONS BY BIO-K+CL1285 ${ }^{\circledR}$ : EXPLANATION OF SOME MECHANISMS OF ACTION

\section{MILLETTE, M FRAPPIER, G ST-PIERRE, É SIMARD, S CARRIÈRE}

Bio-K+ Pharma, Laval, QC

OBJECTIVE: Clinical trials have demonstrated the capacity of a probiotic product named Bio-K+CL1285 ${ }^{\circledR}$ to reduce the incidence of Clostridium difficile infections (CDI) in hospitalized elderly patients receiving antibiotics. However, little is known about the characteristics of this probiotic that could explain its efficacy to prevent CDI. The objective of this project was to determine the anti-C. difficile mechanisms of action of Bio$\mathrm{K}+\mathrm{CL} 1285^{\circledR}$.

METHODS: The agar spot test was used to demonstrate the direct antagonism of the lactobacilli in the product against C. difficile strains but also against other bacteria responsible of nosocomial infections. The presence of antimicrobial metabolites in the probiotic product was determined using cell-free supernatants (CFS) evaluated by well-diffusion assay. Adhesion capacity of Lactobacillus acidophilus CL1285 ${ }^{\circledR}$ and L. casei LBC80R ${ }^{\circledR}$ was measured on Caco-2 and HT-29 cell lines. Also, prevention of the adhesion and cytotoxic effect of C. difficile by probiotic strains or finished product was evaluated in vitro.

RESULTS: Direct inhibition of all C. difficile strains, methicillin-resistant Staphylococcus aureus, vancomycin-resistant enterococci, carbapenemresistant Klebsiella pneumoniae, Escherichia coli O157:H7, Salmonella Typhimurium, Listeria monocytogenes and Bacillus cereus was obtained using each probiotic fermented milk products and pure lactobacilli. Concerning the inhibition capacity of CFS, it was shown that the fermented soy and also the strawberry and fruity products were the most active products. Lactic acid production, differing between the products and the manufacturing time, is thought to be the main antimicrobial metabolite. Lactobacilli and CFS obtained from various finished products were also able to displace C. difficile from cell lines and to reduce cytotoxicity of toxin $\mathrm{A} / \mathrm{B}$ containing C. difficile broth.

CONCLUSION: From these results, it can be concluded that Bio$\mathrm{K}+\mathrm{CL} 1285^{\circledR}$ probiotics could eliminate C. difficile strains via secretion of inhibitory compounds such as lactic acid but can also prevent adhesion of the nosocomial pathogen to intestinal lining. Reduction of the cytotoxic effect by the probiotic product is also discussed.

\section{P18}

DIFFERENTIATION OF MEMBERS OF THE MYCOBACTERIUM TUBERCULOSIS COMPLEX USING A LUMINEX ASSAY IWOLFE $^{1}$, M SHARMA ${ }^{1,2}$, S CHRISTIANSON ${ }^{1}$

${ }^{1}$ Public Health Agency of Canada; ${ }^{2}$ University of Manitoba, Winnipeg, MB

BACKGROUND: Rapid differentiation of the Mycobacterium tuberculosis complex (MTBC) has important clinical and epidemiological applications. Current methods include sequence analysis or probe-based systems such as the GenoType MTBC strip. Sequence analysis can be time consuming, and the GenoType MTBC strips and data can be cumbersome to work with. Molecular differentiation is based largely on single nucleotide polymorphisms (SNPs) within multiple MTBC genes. The Luminex system allows for simultaneous detection of up to 500 probes for specific nucleotide sequences. We have applied this methodology to the identification of the SNPs that are currently used to differentiate MTBC members.

METHODS: PCR primers and Luminex probes were developed that corresponded with SNPs in the gyrB, pncA, 16s rRNA genes along with, region of difference (RD) 1. Probes included each previously observed nucleotide at SNP sites. Probes were detected using the Luminex FlexMap 3D system.

RESULTS: Amplification of the various genes was successful in each of the MTBC members tested. All of the probes were successfully hybridized and detected using the Luminex system. Optimization of conditions shows that the PCR and hybridization conditions lend themselves to multiplexing of these assays.

CONCLUSIONS: The Luminex system can provide high throughput, rapid and accurate differentiation of the MTBC complex with a rapid turnaround time and the ability for multi-plexing.

\section{P19}

\section{COMPARISON OF DIP N COUNT TO STERILE PLASTIC CONTAINERS FOR URINE TRANSPORT C VERMEIREN ${ }^{1,2}$, L BISHOP1 ${ }^{1}$ K KATZ ${ }^{1,2,3}$}

${ }^{1}$ Shared Hospital Laboratory; ${ }^{2}$ University of Toronto; ${ }^{3}$ North York General Hospital, Toronto, ON

OBJECTIVE: The Dip N Count urine paddle (DNC, Starplex, Canada) is a urine collection device for semi-quantitative culture of urine consisting of a dip paddle housing cystine lactose electrolyte deficient agar (CLED) and MacConkey agar (MAC) attached to a screw-top lid and suspended in a plastic vial. In comparison to a sterile plastic container (SPC), the DNC device has several theoretical advantages including decreased growth of contaminants and reduced turn-around time. We compare turn-around time and the contamination rate of urines submitted to our laboratory from two large community teaching hospitals, one using SPC and the other using DNC.

METHODS: The results of all urines submitted for culture between January 2009 and July 2010 from either hospital were retrospectively reviewed. Urines received in SPC were inoculated to CLED and sheep blood agar plates. Urines were processed and results interpreted according to standard protocols. Briefly, isolates from urines collected by a non-invasive method were considered pathogens if the quantity was $\geq 10^{6} \mathrm{cfu} / \mathrm{L}$ and the 
total number of isolates on the plate was $\leq 2$ while $\geq 3$ pathogens were contaminated. Any isolate from urine collected by an invasive method was considered a pathogen. Pearson's chi-squared test was used for statistical calculations.

RESULTS:

\begin{tabular}{|c|c|c|c|c|}
\hline $\begin{array}{l}\text { Collection } \\
\text { Device }\end{array}$ & $\begin{array}{l}\text { No. } \\
\text { Urines }\end{array}$ & $\begin{array}{c}\text { No. Urines } \\
\text { submitted/patient } \\
\text { day }\end{array}$ & $\begin{array}{l}\text { No. Urines with } \\
\text { 'significant' } \\
\text { growth (\%) }\end{array}$ & $\begin{array}{c}\text { No. Contaminated } \\
\text { Urines }\end{array}$ \\
\hline DNC & 19750 & 0.084 & 5297 (27) & $69(0.35)$ \\
\hline SPC & 25883 & 0.071 & $6856(26.5)$ & $228(0.80)$ \\
\hline
\end{tabular}

E.coli, Enterococcus spp. and Klebsiella spp. were the most frequently isolated pathogens, accounting for $61 \%$ and $69 \%$ of specimens with significant growth collected using DNC and SPC respectively. Considering only these pathogens, $72 \%$ and $81 \%(P<0.001)$ were resulted within $48 \mathrm{hrs}$ of receipt in the laboratory using $\mathrm{DNC}$ and $\mathrm{SPC}$, respectively. The contamination rate was significantly lower $(P<0.001)$ for the hospital using DNC compared to the hospital using SPC.

CONCLUSION: In this study we compare urine culture results from two hospitals, each using a different device for transport of urine specimens to the microbiology laboratory. The hospital using DNC had a significantly lower contamination rate. However, for at least the most common pathogens encountered, use of DNC led to delayed results.

\section{P20}

EVALUATION OF A NEW HEALTH CANADA-APPROVED LYME IMMUNOBLOT ASSAY FOR CONFIRMATION OF POSITIVE EIA SCREEN RESULTS

\section{K KADKHODA, P VAN CAESEELE, G SMART, W MAR}

\section{Cadham Provincial Laboratory, Winnipeg, MB}

OBJECTIVE: Lyme disease is a tick-borne infectious disease, which is caused by Borrelia burgdorferi and is found in several provinces of Canada. Diagnosis of Lyme disease is based on multiple factors and criteria with serology being the most important and convenient at the present time which is a two-tiered method: an EIA followed by an Immunoblot. In this study a new Health Canada-approved product for Lyme Immunoblot was evaluated.

METHOD: Sera confirmed as positive for Lyme-specific antibodies by the National Microbiology Laboratory in Winnipeg, MB, were tested by recomLine Borrelia IgG/IgM ${ }^{\mathrm{TM}}$ (Mikrogen) for both Lyme-specific IgM and IgG antibodies based on manufacturer's instructions. Interpretation of immunoblot results were based on sum of the points that each band (if present) received; being $\geq 7$ considered as positive and $\leq 4$ as negative.

RESULTS: Out of 11 sera tested negative by the reference lab, one showed positive and one showed a borderline IgG result while C6 ELISA results for the latter two were highly positive. Furthermore, out of $7 \mathrm{IgG}$ positive sera done by the reference lab, all were positive by our test for IgG. Three serum samples that tested negative for IgM by the reference lab tested positive in our study. This test also did not show any cross-reactivity with sera positive for Syphilis, CMV, EBV, and Mycoplasma IgM.

CONCLUSION: Taken together, using an immunoblot based on wholecell sonicate is inferior to a product with purified recombinant antigens in terms of both specificity and sensitivity in serological diagnosis of Lyme disease. Testing against a gold standard (e.g. PCR) would be a desired approach.

\section{P21}

COMPARISON OF ELUTED SWAB AND AMIES CHARCOAL SWAB FOR DETERMINATION OF METHICILLIN RESISTANT STAPHYLOCOCCUS AUREUS (MRSA) AND VANCOMYCIN RESISTANT ENTEROCOCCUS (VRE) COLONIZATION AND IF NASAL SWAB CULTURE ALONE IS SUFFICIENT FOR DETECTION OF MRSA COLONIZATION IN HOSPITALIZED PATIENTS

$\underline{\text { S JOHN }}{ }^{1}$, B AHMED ${ }^{2}$, L KELLEHER ${ }^{2}$, C KIM ${ }^{2}$, R ONISHI ${ }^{2}$, B MACORETTA ${ }^{2}$, C LEE ${ }^{1,2}$

${ }^{1}$ McMaster University; ${ }^{2} \mathrm{St}$ Joseph's Healthcare, Hamilton, ON OBJECTIVE: 1) To assess whether Eluted swab (ESwab) collection and device, flocked swab and a $1 \mathrm{ml}$ tube of liquid Amies is superior to conventional charcoal swab (CS), in detecting MRSA and VRE colonization. 2) To determine if nare swab alone compared to combination of nare and groin swabs is sufficient for detection of MRSA in colonized patients.

METHODS: Total of 141 nasal and groin and 75 rectal specimens were tested using both ESwab and CS from 47 patients known to be previously colonized with MRSA and 25 with VRE. Aliquots of $100 \mathrm{ul}$ and $10 \mathrm{ul}$ of each specimen suspension were plated on to MRSA and VRE plates. A technologist, blinded to the swabs used, counted colonies after a 24 and $48 \mathrm{hr}$ incubation.

RESULTS: CS detected MRSA in $53 \%$ and $42 \%$ of nare swabs and $51 \%$ and $42 \%$ of groin swabs. CS detected VRE in $73 \%$ and ESwab $75 \%$ from rectal swabs. ESwab yielded $62 \%$ and $59 \%$ of MRSA from nare swabs and $51 \%$ and $42 \%$ from groin swabs. The CS yielded 146 and 88 colony forming units (cfu) when inoculated into 100 and $10 \mathrm{ul}$ respectively. The ESwab yielded 192 and 123 cfu in nares, 151 and 141 cfu in groin and $263 \mathrm{cfu}$ in rectal for 100 and $10 \mathrm{ul}$. The paired t-test indicated superiority to CS swab when inoculated into 100 and $10 \mathrm{ul}$ of liquid Amies for detection of MRSA at $\mathrm{P}<0.00000001$ and $\mathrm{VRE}$ at $\mathrm{P}<0.00000001$.

CONCLUSIONS: ESwab is superior to conventional Amies swab in detecting MRSA and VRE colonization. It may not be necessary to swab both nare and groin, as nare alone is sufficient in detecting 20\% more MRSA colonization.

\section{P22}

COMPARISON OF POOLED ESWABS TO SWABS IN AMIES

TRANSPORT MEDIUM (ATM) FOR DETECTING EPIDEMIOLOGICALLY SIGNIFICANT ORGANISMS (ESO)

FROM SURVEILLANCE IN EMERGENCY ROOM (ER) AND INFLAMMATORY BOWEL DISEASE (IBD) PATIENTS

BM WILLEY ${ }^{1}$, P GNANASUNTHARAM $^{1}$, G NGUYEN ${ }^{1,2}$, B BORGUNDVAAG ${ }^{1,2}$, W LEUNG $^{1,2}$, M LOFTUS $^{1}$, R THANABALAN ${ }^{1}$, V PORTER ${ }^{1}$, G SMALL ${ }^{1}$, IA EDWARDS ${ }^{1}$, P LO $^{1}$, K WONG $^{1}$, G MAHLI ${ }^{1}$, AJ MCGEEER ${ }^{1,2}$, SM POUTANEN ${ }^{1,2}$

${ }^{1}$ Mount Sinai Hospital/University Health Network; ${ }^{2}$ University of Toronto, Toronto, ON

OBJECTIVES: Pilot data comparing single eSwabs to ATM for MRSA and MSSA indicated that eSwab yield fell between ATM direct plating and broth enriched culture. This study compared yield from pooled nasal (NAS) and rectal (REC) eSwabs (PeS) to individual swabs in ATM.

METHODS: With ethics approval, single NAS and REC ATM swabs were taken in parallel to PeS from ER/IBD patients. ATM swabs were plated for MRSA, MSSA, VRE, and resistant (R) enterobacteria (R-GNB: $3^{\text {rd }}$ gen. cephalosporin-R plus $\mathrm{R}$ to quinolones or aminoglycosides). The WASP (Copan) plated $30 \mathrm{uL}$ from each PeS to the same selective media. Incubation and identification methods were standardized.

RESULTS: MRSA were isolated from 7/475 patients [5 (1.1\%) NASATM, 4 (0.8\%) REC-ATM, 6 (1.3\%) NAS-ATM or REC-ATM, 6 (1.3\%) from PeS] with no difference between combined ATM and PeS. MSSA, used as proxy for MRSA, was found in 118 patients [102 (21.5\%) NASATM, 37 (7.9\%) REC-ATM, 112 (23.6\%) NAS-ATM or REC-ATM, and 107 (22.5\%) PeS], also with no difference between combined ATM and PeS (P value $=0.7079$ ). Detection of R-GNB by systems was also not significantly different: REC-ATM and PeS both detected 49 R-GNB from 51 positive patients, respectively, with 47 R-GNB (1 C. freundii, 42 E. coli, 
4 K. pneumoniae, 4 P. mirabilis) identified from both REC-ATM and PeS, while E. coli from 4 remaining patients were identified from REC-ATM (2 isolates) or PeS (2 isolates) only. No VRE were identified from any swab.

CONCLUSIONS: This study found the WASP planted PeS (nasal plus rectal) to have an equivalent yield of ESO compared to NAS-ATM and REC-ATM planted separately.

\section{P23 \\ CLINICAL EVALUATION OF FIVE CHROMOGENIC SELECTIVE AGAR FORMULATIONS FOR THE DETECTION OF VanB-MEDIATED RESISTANCE}

FULLER, L TURNBULL

University of Alberta Hospital, Edmonton, AB

OBJECTIVE: VanB vancomcycin-resistant enterococcal (VRE) detection using selective agar is difficult due to the low level resistance conferred by these orgainisms. We evaluated five commercial selective media for VanB VRE detection in a clinical setting.

METHODS: In parallel with our reference $\mathrm{m}$-Enterococcosel agar (m-Ent), we tested 270 rectal swabs and 30 stool specimens inoculated with $\operatorname{vanB}$ VRE; the latter specimens were set up to reflect representative concentrations in stool ( 10e5 CFU per swab). Plates were read at 24 and $48 \mathrm{~h}$ as no growth, negative (non-chromogenic), and positive (chromogenic). Test media included VRESelect (Bio-Rad), Colorex Red and Colorex Blue (CHROMagar), Brilliance VRE (Oxoid), and chromID (bioMerieux).

RESULTS: The 24-h VanB sensitivity ( $\mathrm{n}=30$ ) was 27\%, 57\%, 83\%, 63\%, and $43 \%$ for VRESelect, Colorex Red, Colorex Blue, Brilliance VRE, and chromID, respectively; the 48-h sensitivities achieved or neared $100 \%$ for all challenge media and $\mathrm{m}$-Ent was $83 \%$. The 24 -h specificity $(\mathrm{n}=270)$ was 96\%, 97\%, 92\%, 93\%, and 99\% for VRESelect, Colorex Red, Colorex Blue, Brilliance VRE, and chromID, respectively, and all values decreased at least $6 \%$ with $48 \mathrm{~h}$ incubation (chromID not read at $48 \mathrm{~h}$ ); m-Ent specificity was $97 \%$ at 48 hr. A majority of non-specific (chromogenic) growth on all test media was identified as vancomycin susceptible enterococcus (VSE); none of these grew on BHI vancomycin agar $(6 \mathrm{mg} / \mathrm{L})$.

CONCLUSIONS: Overall, the five test media showed enhanced VanB VRE detection at $24 \mathrm{~h}$ incubation, particularly the Colorex Blue (CHROMagar), indicating the potential for more timely communication of results.

\section{P24}

RETROSPECTIVE EVALUATION OF OXOID BRILLIANCE VRE ${ }^{\circledR}$ AND BIO-RAD VRESELECT ${ }^{\circledR}$ CHROMOGENIC AGARS

FOR DETECTION OF VANCOMYCIN-RESISTANT ENTEROCOCCI (VRE) USING A GENETICALLY DIVERSE ISOLATE COLLECTION

BM WILLEY ${ }^{1}$, P GNANASUNTHARAM ${ }^{1}$, L LOUIE ${ }^{2}$, K PRAYITNO $^{3}$, T FUNG ${ }^{3}$, P GNANASUNTHARAM $^{1}$, P LO $^{1}$, AJ MCGEER ${ }^{1,3}$, SM POUTANEN ${ }^{1,3}$

${ }^{1}$ Mount Sinai Hospital/University Health Network; ${ }^{2}$ Sunnybrook Health Sciences Centre; ${ }^{3}$ University of Toronto, Toronto, ON

OBJECTIVES: Prospective evaluations of VRE selective agars are limited due to the clonality of circulating VRE. This study compared chromogenic to conventional VRE agars using diverse VRE and non-VRE clinical isolates.

METHODS: Brilliance VRE (BRL), VRESelect (SLT), Bile Esculin Azide-V (BEAV) and Difco mEnterococcus-V (mEV) were studied using 137 strains: 49 E. faecium [EFE: 43 VR - 24 vanA, 17 vanB, 1 vanD; $6 \mathrm{~V}$-susceptible (VS)], 44 E. faecalis [EFC: $26 \mathrm{VR}-2$ vanA, 17 vanB, 6 vanE, 1 vanL; $18 \mathrm{VS}$, 15 E. gallinarum (GAL), 8 E. casseliflavus (CAS), 10 Leuconostoc, 7 Pediococcus, 4 Lactobacillus. Plates were inoculated with $100 \mathrm{uL}$ of each $0.5 \mathrm{McF}$ arland suspension, and read after $24 \mathrm{~h}$ and $48 \mathrm{~h}$ $(\mathrm{mEV}$ at $72 \mathrm{~h})$ at $37 \mathrm{C}$ for colony no., size and colour. Data from all lots for each agar were pooled and converted to $\%$ to be comparable.

RESULTS: Overall data from BRL, mEV, BEAV and SLT tested with 548, 411,411 and 274 isolates, respectively, is summarized below.

\begin{tabular}{|c|c|c|c|c|c|c|c|c|c|}
\hline \multirow{2}{*}{$\begin{array}{l}\begin{array}{l}\text { VRE agar } \\
\text { [no. of lots tested] }\end{array} \\
\text { Examination time point }\end{array}$} & \multicolumn{2}{|c|}{$\begin{array}{l}\text { Oxoid Brilliance VRE } \\
\text { [4 lots] }\end{array}$} & \multicolumn{2}{|c|}{$\begin{array}{l}\text { Bio. Rad VRESelect } \\
\text { [2 lots] }\end{array}$} & \multicolumn{2}{|c|}{$\begin{array}{c}\text { Oxoid BEAV } \\
\text { [ } 3 \text { lots| }\end{array}$} & \multicolumn{3}{|c|}{$\begin{array}{c}\text { mEnterococcus+Vancomycin } \\
{[3 \text { lots] }}\end{array}$} \\
\hline & $24 \mathrm{~h}$ & $48 \mathrm{~h}$ & $24 \mathrm{~h}$ & $48 \mathrm{~h}$ & $24 \mathrm{~h}$ & 8h & $24 \mathrm{~h}$ & $48 \mathrm{~h}$ & $72 \mathrm{~h}$ \\
\hline & $\begin{array}{l}99.1 \\
899.97)\end{array}$ & $\begin{array}{c}100 \\
98.1-100)\end{array}$ & \begin{tabular}{|c|}
90 \\
(63.2.94.3)
\end{tabular} & $\begin{array}{c}99.2 \\
\beta 5 .>99.99) \\
\end{array}$ & $\begin{array}{c}93.3 \\
(80.696 .3) \\
\end{array}$ & $(35.9$ & & $\begin{array}{l}95.6 \\
397.9)\end{array}$ & \\
\hline Sensitivi & $\begin{array}{c}94.1 \\
(90.696 .4)\end{array}$ & \begin{tabular}{|c|}
97.8 \\
$95.299 .1)$
\end{tabular} & $\begin{array}{c}82.4 \\
(P 5.87 .9)\end{array}$ & $\begin{array}{c}91.9 \\
(86.95 .6)\end{array}$ & $\begin{array}{c}90.7 \\
(95.8 .94)\end{array}$ & & $\begin{array}{c}60.8 \\
(53.967 .2)\end{array}$ & $\begin{array}{c}87.3 \\
(81.991 .2)\end{array}$ & $\begin{array}{c}92.2 \\
(87.695 .2)\end{array}$ \\
\hline & $\begin{array}{c}96.8 \\
95.2 .99 .1)\end{array}$ & $\begin{array}{c}94.6 \\
01.2 .96 .8)\end{array}$ & \begin{tabular}{|c|}
82.6 \\
$75.388 .1)$
\end{tabular} & $\begin{array}{c}60.9 \\
{[52.5-68.6)}\end{array}$ & $\begin{array}{c}60.9 \\
(54.167 .3)\end{array}$ & $\begin{array}{c}59.9 \\
(53.1 .66 .3)\end{array}$ & $\begin{array}{c}81.2 \\
(76.385 .2)\end{array}$ & $\begin{array}{c}75.4 \\
(69.180 .8)\end{array}$ & $\begin{array}{l}64.7 \\
(58-70.9)\end{array}$ \\
\hline
\end{tabular}

CONCLUSIONS: While BRL had 24h performance that exceeded all other agars combined, this study indicates that as with other VRE selective agars, BRL requires $48 \mathrm{~h}$ to grow $100 \%$ of vanA/B VRE isolates.

\section{P25}

PROSPECTIVE COMPARISON OF OXOID BRILLIANCE VRE ${ }^{\circledR}$ AND BIO-RAD VRESELECT ${ }^{\circledR}$ TO NON-CHROMOGENIC AGARS FOR DETECTING VANCOMYCIN-RESISTANT ENTEROCOCCI (VRE) FROM SURVEILLANCE SPECIMENS BM WILLEY ${ }^{1}$, L LOUIE ${ }^{2}$, P GNANASUNTHARAM $^{3}$, T FUNG $^{3}$, C WATT $^{2}$, P GNANASUNTHARAM ${ }^{1}$, C VERMEIREN ${ }^{4}$, G RICCI $^{5}$, P LO $^{1}$, K WONG ${ }^{1}$, A SIMOR ${ }^{2}$, AJ MCGEER ${ }^{1,2}$, T MAZZULLI ${ }^{1,2}$, SM POUTANEN 1,2

${ }^{1}$ Mount Sinai Hospital/University Health Network; ${ }^{2}$ Sunnybrook Health Sciences Centre; ${ }^{3}$ University of Toronto; ${ }^{4}$ Shared Hospital Laboratory; ${ }^{5}$ William Osler Health Sciences Centre, Toronto, ON OBJECTIVE: To improve turn-around-time (TAT) to VRE isolation without loss of sensitivity, this study compared new chromogenic to conventional VRE agars.

METHODS: 3000 prospective specimens were plated to: Brilliance VRE (BRL); VRESelect (SLT); Bile Esculin Azide-V (BEAV); and $\mathrm{mEnterococcus}-\mathrm{V}(\mathrm{mEV})$. All were read at $24 \mathrm{~h} / 48 \mathrm{~h}$, and $\mathrm{mEV}$ at $72 \mathrm{~h}$. Only potential E. faecium/E. faecalis (BRL: purple/blue, SLT: pink/aqua) were identified (ID), direct from agars if possible; $\mathrm{G}+\mathrm{C}$ from $\mathrm{mEV} / \mathrm{black}$ colonies from BEAV were ID via BA subs. ID was by PYR, arabinose, MGP, ampicillin disc, BHIV6 screen. VRE were PCR confirmed.

RESULTS: The 141 VRE from 140 specimens [137/2926 rectal swabs, 3/74 stools] included 126 vanA E. faecium (EFE), 11 vanB EFE and 4 vanB E. faecalis. mEV detected 140, BRIL 137, SLT 135 and BEAV 124. NonVRE requiring tests to rule out VRE included: BRIL (527), mEV (646), BEAV (718) and SLT (804). Use of either BRL or SLT improved TAT significantly (BRL vs. $\mathrm{mEV} / \mathrm{BEAV}(\mathrm{P}=0.003 / 0.001)$ and $\mathrm{SLT}$ vs. $\mathrm{mEV} /$ $\operatorname{BEAV}(\mathrm{P}=0.03 / 0.01)$. Performance, $(95 \% \mathrm{CI})$, is below.

\begin{tabular}{|l|c|c|c|c|}
\hline $\begin{array}{l}\text { Performance at } \\
\text { read time }\end{array}$ & $\begin{array}{c}\text { Oxoid } \\
\text { Brilliance VRE Agar }\end{array}$ & $\begin{array}{c}\text { Bio-Rad } \\
\text { VRESelect Agar }\end{array}$ & $\begin{array}{c}\text { mEnterococcus Vancomycin } \\
\text { Agar }\end{array}$ & $\begin{array}{c}\text { Bile Esculin Azide Vancomycin } \\
\text { Agar }\end{array}$ \\
\hline Sensitivity at 24h & $\begin{array}{c}92.9 \% \\
(87.3-96.3)\end{array}$ & $\begin{array}{c}90.1 \% \\
(83.9-94.1)\end{array}$ & $\begin{array}{c}80.1 \% \\
(72.8-85.9)\end{array}$ & $\begin{array}{c}78.7 \% \\
(71.2-84.7)\end{array}$ \\
\hline Sensitivity at 48h & $\begin{array}{c}97.2 \% \\
(92.7-99.1)\end{array}$ & $\begin{array}{c}95.8 \% \\
(90.8-98.2)\end{array}$ & $\begin{array}{c}96.5 \% \\
(91.8-98.7)\end{array}$ & $\begin{array}{c}87.9 \% \\
(81.5-92.4)\end{array}$ \\
\hline Sensitivity at 72h & ND & ND & $\begin{array}{c}99.3 \% \\
(95.7-999.9)\end{array}$ & ND \\
\hline Specificity at 24h & $\begin{array}{c}88.8 \% \\
(87.6-89.9)\end{array}$ & $\begin{array}{c}79.4 \% \\
(77.9-80.9)\end{array}$ & $\begin{array}{c}92.4 \% \\
(91.5-93.4)\end{array}$ & $\begin{array}{c}84.9 \% \\
(83.6-86.2)\end{array}$ \\
\hline Specificity at 48h & $\begin{array}{c}79.8 \% \\
(78.3-81.4)\end{array}$ & $\begin{array}{c}72.0 \% \\
(70.4-73.7)\end{array}$ & $\begin{array}{c}83.5 \% \\
(82.2-84.8)\end{array}$ & $\begin{array}{c}70.9 \% \\
(69.2-72.7)\end{array}$ \\
\hline Specificity at 72h & ND & ND & $\begin{array}{c}78.3 \% \\
(76.8-79.8)\end{array}$ & ND \\
\hline
\end{tabular}

CONCLUSIONS: Compared to conventional agar, use of either chromogenic agar significantly improved TAT to VRE detection with minimal loss of sensitivity.

\section{P26}

EVALUATION OF THREE CHROMOGENIC MEDIA FOR DETECTION OF VANCOMYCIN-RESISTANT ENTEROCOCCI IN A TERTIARY-CARE HOSPITAL

M MILLER ${ }^{2}$, D ZOUTMAN ${ }^{1,2}$, L TOMALTY ${ }^{1,2}$, D ARMSTRONG ${ }^{2}$, J DIEN BARD ${ }^{1,2}$

${ }^{1}$ Queen's University; ${ }^{2}$ Kingston General Hospital, Kingston, ON OBJECTIVE: To evaluate the performance of Brilliance VRE Agar, Colorex VRE Agar and VRE Select Agar for detecting vancomycinresistant enterococci (VRE) from faecal samples and rectal swabs collected from patients undergoing VRE-screening for VRE colonization at a tertiary care hospital.

METHODS: 95 rectal swabs/stool specimens (22 positives and 73 negatives) were collected from patients admitted to Kingston General Hospital 
to screen for VRE colonization. Swabs or stools were inoculated into BHI enrichment broth and incubated overnight at $35-37^{\circ} \mathrm{C}$, followed by streaking $10 \mu \mathrm{L}$ of broth onto Brilliance VRE Agar, Colorex VRE Agar and VRE Select Agar. All plates were incubated at $35-37^{\circ} \mathrm{C}$ for $22 \mathrm{~h}, 24 \mathrm{~h}$ and $26 \mathrm{~h}$. Suspicious VRE colonies were confirmed by Gram stain, biochemical identification, and antibiotic susceptibility testing. Any Enterococcus species that were confirmed to have a minimum inhibitory concentration (MIC) of $\geq 8 \mu \mathrm{g} / \mathrm{mL}$ for vancomycin were considered VRE positive.

RESULTS: Of the three chromogenic agars, the Brilliance VRE Agar was able to identify all 22 positives ( $100 \%$ sensitivity), followed by Colorex VRE Agar with 21 positives (96\% sensitivity). The one false negative VRE grew only 1 colony on the Brilliance VRE Agar at $26 \mathrm{~h}$. The VRE Select Agar had the lowest sensitivity with 15 true positives (68\%) identified. All three agars required $26 \mathrm{~h}$ incubation to recover all VRE positive isolates. Although the Brilliance VRE Agar had 100\% sensitivity, the specificity was $71 \%$ with 21 false positives identified. The majority of the false positives (18/21) were detected at $24 \mathrm{~h}$. The Colorex VRE Agar and VRE Select Agar had very similar specificities of $90 \%$ and $92 \%$, respectively.

CONCLUSION: Brilliance VRE Agar and Colorex VRE Agar showed exceptionally better sensitivity than VRE Select Agar. In contrast, Colorex VRE Agar and VRE Select Agar had much higher specificity than Brilliance VRE Agar. When taking into account the overall performances of the agar plates to accurately identify VRE isolates in broth-enriched rectal swabs and stool samples, the Colorex VRE Agar appears to be the most highly effective screening agar.

\section{P27}

\section{BIOFILM SUSCEPTIBILITY OF P AERUGINOSA ISOLATED FROM PATIENTS WITH OTITIS MEDIA TO CIPRODEX, CIPROFLOXACIN AND N-ACETYLCYSTEINE} M NOBLE ${ }^{1}$, V RESTELLI ${ }^{1}$, R RENNIE ${ }^{1,2}$, B WESTERBERG ${ }^{1}$, L TURNBULL ${ }^{1,2}$

${ }^{1}$ University of British Columbia, Vancouver, BC; ${ }^{2}$ University of Alberta, Edmonton, AB

OBJECTIVE: $P$. aeruginosa is a common cause of chronic otitis media in which biofilm formation favours bacterial resistance. N-Acetylcysteine (NAC) is a mucolytic agent with clinical synergy with Ciprodex (ciprofloxacin $0.3 \% /$ dexamethasone $0.1 \%$ ) for treatment of otitis media. Our objective was to demonstrate synergy between Ciprodex and or ciprofloxacin and NAC against biofilm forming P. aeruginosa strains isolated from otitis cases.

METHODS: 15 clinical isolates of P. aeruginosa were tested using the bioFILM PA (tm) antimicrobial susceptibility assay (Innovotech, Edmonton, $\mathrm{AB}$ ) to determine antimicrobial susceptibility of both planktonic and biofilm $P$. aeruginosa to Ciprodex and ciprofloxacin both alone and in combination with NAC. Isolates were grown on plastic pegs to allow identical biofilms to form. The pegs were then placed into 96 well microplates containing Ciprodex and ciprofloxacin both alone or in combination with NAC at various concentrations. Susceptibility of the planktonic population was measured by a microreader. Viability of the sessile population was assessed after $24 \mathrm{hrs}$.

RESULTS: Of the 15 P. aeruginosa strains, one (7\%) showed growth in all Ciprodex tested concentrations and two strains (13\%) showed growth in two ciprofloxacin concentrations in the planktonic state. Higher resistance to Ciprodex and ciprofloxacin was observed in the sessile population where five $(33 \%)$ strains showed growth in one or more ciprodex or ciprofloxacin concentration. NAC, in a final concentration of $0.5 \mathrm{ug} / \mathrm{mL}$, effectively inhibited both planktonic and sessile populations when added to any of the Ciprodex or ciprofloxacin concentrations. No growth was observed in any of the wells with added NAC.

CONCLUSION: Sessile P. aeruginosa were less susceptible than the planktonic populations to Ciprodex and ciprofloxacin when tested alone. The addition of NAC completely inhibited growth of both planktonic and sessile populations. These results suggest there are differences between biofilm and planktonic state in P. aeruginosa strains. Biofilm P. aeruginosa are more resistant to Ciprodex or ciprofloxacin. The combination of these agents with NAC may increase their clinical utility in the treatment of chronic otitis media.

\section{P28}

SCREENING POSSIBLE CANDIDA ALBICANS ISOLATES FOR CANDIDA DUBLINIENSIS: IS IT WORTH IT?

\section{HALDANE}

${ }^{1}$ Queen Elizabeth II Health Sciences Centre; ${ }^{2}$ Dalhousie University, Halifax, NS

OBJECTIVE: To determine the added value of screening possible Candida albicans isolates for Candida dubliniensis, with reference to the site of isolation, frequency and detection of fluconazole resistant isolates.

METHODS: Laboratory records were reviewed retrospectively from 2005 to 2010. Candida albicans from non sterile sites was presumptively identified by using chromogenic media. These isolates, and C. albicans isolates from sterile sites identified biochemically, were screened to detect Candida dubliniensis using the inability to grow at $42^{\circ} \mathrm{C}$ and abundant production of chlamydospores on tobacco agar. Isolates testing positive were confirmed biochemically using the API 20C AUX (bioMerieux). Susceptibility to fluconazole was determined by disc diffusion using the CLSI method.

RESULTS: There were 15 isolates from 11 patients (1 patient had 4 isolates, 1 patient had 2, the remainder had a single isolate). 14 isolates were from oral mucosa, 1 was from a central line. All of the isolates were susceptible to fluconazole.

CONCLUSIONS: Routine sceening of all Candida albicans isolates to rule out Candida dubliniensis is not warranted. More directed screening, for example by screening of resistant isolates, might be a better use of resources.

\section{P29}

EVALUATION OF VITEK-2 (VT2) YS01 ANTIFUNGAL SUSCEPTIBILITY TESTING (AFST) IN CANDIDA SPECIES BM WILLEY ${ }^{1}$, K PRAYITNO ${ }^{2}$, A GELOSIA ${ }^{1}$, C PORTER ${ }^{1}$, J FULLER ${ }^{3}$, SM POUTANEN ${ }^{1,2}$

${ }^{1}$ Mount Sinai Hospital/University Health Network; ${ }^{2}$ University of Toronto, Toronto, ON; ${ }^{3}$ University of Alberta Hospital, Edmonton, AB BACKGROUND: Broth microdilution (BMD) AFST for Candida is not routine and yet timely AFST is important in patient care. This study evaluated the YSO1 AFST card on the automated VT2 using a reference collection.

METHODS: VT2 YS01 AFST results from 169 Candida (70 C. albicans, 48 C. glabrata, 16 C. krusei, 12 C. tropicalis, 11 C. parapsilosis, 10 C. kefyr, 2 C. lusitaniae) were compared with $48 \mathrm{~h}$ fluconazole (FLU), voriconazole (VOR), 5-flucytosine (5FC) and amphotericin B (AmB) BMD MICs using Cumitech 31A standards. Essential agreement (EA), category agreement (CA), major (ME), very major (VME) and minor ( $\mathrm{mE}$ ) errors were calculated using FLU, VOR and 5FC CLSI breakpoints and NS >1 for AmB. RESULTS: AFST results for the 169 Candida spp. tested are tabled below.

\begin{tabular}{|c|c|c|c|c|c|c|c|c|c|}
\hline \multirow[t]{2}{*}{ Drug } & \multicolumn{6}{|c|}{ No. $(\%) /[95 \% \mathrm{Cl}]$} & \multicolumn{3}{|c|}{$\%[95 \% \mathrm{Cl}]$} \\
\hline & $s$ & SDD $\|$ & R/NS & VME & ME & $\mathrm{mE}$ & $\pm 1 E A$ & $\pm 2 \mathrm{EA}$ & $\mathrm{CA}$ \\
\hline FLU & $\begin{array}{l}130 \\
(77)\end{array}$ & $\begin{array}{c}22 \\
(13)\end{array}$ & $\begin{array}{l}17 \\
(10)\end{array}$ & $\begin{array}{l}2(12) \\
{[2-36]}\end{array}$ & $\begin{array}{l}0(0) \\
{[0.3]}\end{array}$ & $\begin{array}{l}29(17) \\
{[12-24]}\end{array}$ & $\begin{array}{c}86 \\
{[80-90]}\end{array}$ & $\begin{array}{c}94 \\
{[89-97]}\end{array}$ & $\begin{array}{c}82 \\
{[75-87]}\end{array}$ \\
\hline VOR & $\begin{array}{l}153 \\
(90)\end{array}$ & $\begin{array}{c}9 \\
\text { (5) }\end{array}$ & $\begin{array}{l}7 \\
\text { (4) }\end{array}$ & $\begin{array}{l}2(29) \\
{[8-65]}\end{array}$ & $\begin{array}{l}0(0) \\
{[0.3]}\end{array}$ & $\begin{array}{c}7(4.1) \\
{[2-8]}\end{array}$ & $\begin{array}{c}88 \\
\text { [82-92] }\end{array}$ & $\begin{array}{c}96 \\
{[92-98]}\end{array}$ & $\begin{array}{c}95 \\
\text { [90-97] }\end{array}$ \\
\hline 5FC & $\begin{array}{l}142 \\
\{84\}\end{array}$ & $\begin{array}{l}14 \\
(8)\end{array}$ & $\begin{array}{l}13 \\
\text { (8) }\end{array}$ & $\begin{array}{c}0(0) \\
{[0-27]}\end{array}$ & $\begin{array}{l}0(0) \\
{[0.3]}\end{array}$ & $\begin{array}{c}9(5.3) \\
{[3.10]} \\
\end{array}$ & $\begin{array}{c}93 \\
{[88-96]}\end{array}$ & $\begin{array}{c}98 \\
{[95-100]}\end{array}$ & $\begin{array}{c}95 \\
{[90-97]}\end{array}$ \\
\hline AmB & $\begin{array}{l}149 \\
(88)\end{array}$ & - & $\begin{array}{l}20 \\
\text { (12) }\end{array}$ & $\begin{array}{l}4(20) \\
{[8-42]}\end{array}$ & $\begin{array}{c}16 \text { (11) } \\
7-17]\end{array}$ & - & $\begin{array}{c}84 \\
{[78-89]}\end{array}$ & $\begin{array}{c}99 \\
{[96-100]}\end{array}$ & $\begin{array}{c}88 \\
\text { [82.92] }\end{array}$ \\
\hline \multicolumn{4}{|c|}{ Cumitech 31 A limits: } & $3 \%$ & $3 \%$ & $\leq 7 \%{ }^{*}$ & $\geq 90$ & . & $\geq 90$ \\
\hline
\end{tabular}

CONCLUSIONS: Only VT2 YS01 5FC met standards, while FLU (mE, $\mathrm{CA}$ ), VOR (VME), and AmB (VME, ME, EA \pm 1 ) did not. Further study of the VT2 YSO1 with more $\mathrm{R}$ isolates and parallel BMD/VT2 testing is needed. 
ROOM: RAMEZAY

\section{P30}

DETERMINING PRESENCE OF BROAD SPECTRUM
BETA-LACTAMASES IN ENTEROBACTERIACEAE -
COMPARISON OF THE MAST DISC METHOD TO
STANDARD PHENOTYPIC AND MOLECULAR DETECTION
METHODS

M HOOPER ${ }^{1}$, V HADWELL ${ }^{2}$, L HOANG ${ }^{3,4}$, A BALBIRNIE ${ }^{5}$, E BLONDEL-HILL 2,3

${ }^{1}$ University of Victoria, Victoria; ${ }^{2}$ Kelowna General Hospital, Kelowna; ${ }^{3}$ University of British Columbia; ${ }^{4} \mathrm{BC}$ Centre for Disease Control, Vancouver, BC; ${ }^{5}$ Simon Fraser University, Burnaby, BC OBJECTIVES: Detection of plasmid mediated broad spectrum beta-lactamases (BSBL) including extended spectrum beta-lactamases (ESBL), and AmpC cephalosporinases (AmpC) is important for both therapeutic and epidemiological purposes. This study compared the performance of the MAST disc method for BSBL detection to standard phenotypic detection. Molecular confirmation was based on the most common gene targets for transmissible beta-lactamases.

METHODS: Screen positive cefotaxime resistant Enterobacteriaceae $(\mathrm{N}=82)$ were tested using the novel MAST disc method to detect ESBLs, AmpCs or both. Standard phenotypic tests included ESBL confirmatory discs and AmpC Etest ${ }^{\circledR}$ method. All isolates were blindly tested for multiple transmissible beta-lactamase gene targets.

RESULTS: Full agreement of all 3 methods was found for 55 of the isolates (30 AmpC, 16 ESBL, 1 ESBL and AmpC, 8 no resistance). An additional 21 isolates demonstrated AmpC or ESBL by phenotypic and MAST methods, but were negative by molecular method, as the potential genes were not included in the PCR panel. Five isolates were inconclusive by AmpC Etest ${ }^{\circledR}$ but positive by MAST and molecular method. One isolate was ESBL positive by phenotypic and molecular method while further work was recommended by the MAST method.

CONCLUSIONS: With the exception of one organism requiring further testing, the MAST test was able to identify BSBLs in all isolates tested.

\section{P31}

\section{DETECTION OF PROLYLIMINOPEPTIDASE-NEGATIVE} NEISSERIA GONORRHOEAE STRAINS IN QUÉBEC

B LEFEBVRE ${ }^{1}$, F LAMOTHE ${ }^{2}$, M MILLER ${ }^{3}$, C FORTIN ${ }^{4}$, I MARTIN ${ }^{5}$, LK NG $^{5}$, A-M BOURGAULT ${ }^{1}$

${ }^{1}$ Laboratoire de santé publique du Québec, Sainte-Anne-de-Bellevue; ${ }^{2} \mathrm{CHUM}$-Hôpital St-Luc; ${ }^{3}$ Hôpital général juif SMBD; ${ }^{4} \mathrm{CHUM}$-Hopital Notre-Dame, Montréal, QC; ${ }^{5}$ National Microbiology Laboratory, Winnipeg, MB

OBJECTIVES: To detect the occurrence of Neisseria gonorrhoeae (NG) strains failing to produce prolyliminopeptidase enzyme (PIP) that could be undetected by commercial test panels.

METHODS: From 05/2007 to 11/2010, 724 NG strains isolated in three laboratories in Montreal and identified by carbohydrate utilization tests were tested by commercial kits using PIP for NG identification: API-NH, Rapid ID-NH and Gonochek II. Pulsed field gel electrophoresis (PFGE), pip gene sequencing and NG multiantigen sequence typing (NG-MAST) were performed on the first 24 PIP-negative isolates.

RESULTS: Thirty (4.1\%) isolates were PIP-negative: 0/120 in 2007, $1 / 186$ in 2008, 11/207 in 2009 and 18/211 in 2010. All strains were isolated in males residing in the Montreal area. The identification obtained with API-NH was NG (94.6\%), with Rapid ID-NH, Kingella kingae (98.4\%) and with Gonochek II, Moraxella catarrhalis. The package inserts recommended additional tests and when performed, the kits provided correct identifications. Sequencing of the pip gene revealed a deletion mutation (nucleotide 110), possibly resulting in a truncated protein. Twenty strains were assigned identical NG-MAST sequence type ST-210, 3 strains as ST-292 and 1 strain as ST-4638. According to PFGE analysis, PIP-negative strains were all related and different from PIP-positive strains isolated.
CONCLUSIONS: This study reiterates the importance of surveillance programs using "gold standards" and of following the manufacturers instructions in order to detect NG variants.

\section{P32}

EVALUATION OF THE MULTI-LOCUS VARIABLE NUMBER TANDEM REPEAT ANALYSIS (MLVA) PULSENET PROTOCOL FOR TYPING SALMONELLA ENTERITIDIS

AK KEARNEY ${ }^{1}$, L HOANG ${ }^{2,3}$ M TAYLOR $^{4}$, E GELANIS ${ }^{4,5}$, A GERBASI ${ }^{1}$, M GILMOUR ${ }^{1,6}$

${ }^{1}$ National Microbiology Laboratory, Winnipeg, MB; ${ }^{2}$ BCCDC Public Health and Reference Microbiology Laboratory, PHSA; ${ }^{3}$ Department of Pathology and Laboratory Medicine, University of British Columbia, Vancouver, BC; ${ }^{4} \mathrm{BC}$ Centre for Disease Control; ${ }^{5}$ University of British Columbia, Vancouver, BC; ${ }^{6}$ Department of Medical Microbiology and Infectious Diseases, University of Manitoba, Winnipeg, MB

OBJECTIVE: Canadian surveillance systems have identified that Salmonella enterica serovar Enteritidis accounts for approximately $40 \%$ of all human clinical Salmonella isolates. The US CDC has recently developed an MLVA protocol for S. Enteritidis. Prior to the widespread implementation of MLVA in PulseNet Canada, this study sought to evaluate the subtyping results achieved by this method on a panel of Canadian isolates.

METHODS: MLVA was carried out on some of the most common PT and PFGE pattern combinations seen in Canada including: PT8/SENXAI.0003 and PT13/SENXAI.0038. VNTR loci were amplified in two multiplex PCR reactions and characterized by capillary gel electrophoresis using an ABI 3130xl. Fragment sizes were determined using GeneMapper software and Applied Maths Bionumerics.

RESULTS: This MLVA scheme corroborated the subtype lineages previously seen across the $S$. Enteritidis population through PT and PFGE, and in some instances elucidated additional diversity. Conversely, there were major MLVA types that each contained multiple PT and PFGE types, suggesting that in some cases MLVA is less discriminatory than the current methods. CONCLUSIONS: S. Enteritidis has already been established as a clonal serovar, and owing to the small proportion of total genome sampled by MLVA, it is possible that this method may not be the most appropriate method to routinely subtype isolates. However, MLVA will possibly still be suitable as a supplementary method during outbreak investigations and other similar investigations into short-term evolution.

\section{P33}

GENETIC HETEROGENEITY OF COMMUNITY ASSOCIATED METHICILLIN-RESISTANT STAPHYLOCOCCUS AUREUS IN SASKATCHEWAN

PN LEVETT $^{1}{ }^{1}$, RR MCDONALD $^{1}{ }^{1}$, E NAGLE $^{1}$, CE WONG $^{1}$,

M MULVEY $^{2}$, GB HORSMAN1; Comité sur les infections nosocomiales du Québec

${ }^{1}$ Saskatchewan Disease Control Laboratory, Regina, SK; ${ }^{2}$ National Microbiology Laboratory, Winnipeg, MB

OBJECTIVE: The initial outbreak in Saskatchewan of communityassociated methicillin-resistant Staphylococcus aureus (CA-MRSA) occurred in the fall of 2000. The previously un-recognized CA-MRSA strain, designated CMRSA7, was found to be consistent with the USA400 cluster, first recovered in the mid-1990s. This cluster was subsequently hypothesised to have spread from Minnesota to Saskatchewan via Manitoba. The objective of this study was to investigate the genetic heterogeneity of CMRSA7 isolates from Saskatchewan.

METHODS: CMRSA7 isolates $(n=1557)$ from Saskatchewan during the period 2006-2010 were characterised by spa typing. The presence of genes coding for Panton-Valentine leukocidin was detected using PCR.

RESULTS: CMRSA7 represented over 50\% of all MRSA isolates recovered in Saskatchewan. 26 spa types belonging to the CMRSA7 cluster were detected during the period studied, of which 9 were first detected in Saskatchewan. The most common spa type throughout the period studied was t128 (79\%). Most CMRSA7 strains were PVL positive but there was a 
trend towards decreasing prevalence of PVL gene positivity. In contrast, only $4 \%$ of spa type 1508 isolates harboured PVL-encoding genes. This spa type was uncommon among 2006 isolates, but appeared to be increasing in relative frequency ( $4 \%$ in 2006 vs $18 \%$ in 2010).

CONCLUSIONS: CMRSA7 strains are genetically heterogeneous and this trend may be increasing. Non-toxigenic strains are increasing in frequency, and detection of PVL genes is no longer a reliable marker of CMRSA7 isolation.

\section{P34}

CHARACTERIZATION OF METHICILLIN-RESISTANT STAPHYLOCOCCUS AUREUS (MRSA) ISOLATES FROM CANADIAN PATIENTS WITH BLOODSTREAM INFECTION

(BSI)

A MOUNCHILI ${ }^{1}$, A SIMOR ${ }^{2}$, D GRAVEL ${ }^{1}$, M MULVEY $^{3}$, E BRYCE $^{4}$,

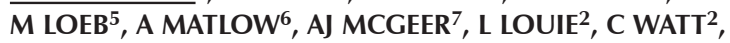
J CAMPBELL ${ }^{3}$; Canadian Nosocomial Infection Surveillance Program $^{1}$

${ }^{1}$ Centre for Communicable Diseases and Infection Control, Public Health Agency of Canada; ${ }^{2}$ Sunnybrook Health Sciences Centre, Ottawa, ON; ${ }^{3}$ National Microbiology Laboratory, Public Health Agency of Canada, Winnipeg, MB; ${ }^{4}$ Vancouver General Hospital, Vancouver, BC; ${ }^{5}$ Hamilton Health Sciences Corporation, Hamilton; ${ }^{6}$ Hospital for Sick Children; ${ }^{7}$ Mount Sinai Hospital, Toronto, ON OBJECTIVE: To characterize MRSA recovered from blood cultures of patients identified during prospective surveillance conducted by the Canadian Nosocomial Infection Surveillance Program in 2008 and 2009. METHODS: Surveillance for MRSA bloodstream infections (BSI) cases was done in about 50 Canadian hospitals in 2008 and 2009. Medical records were reviewed for clinical and epidemiologic data. A single blood culture isolate from each patient was characterized by antimicrobial susceptibility testing, pulsed-field gel electrophoresis (PFGE), and spa typing. RESULTS: The rates of MRSA BSI were 0.50 per 1,000 admissions in 2008 and 0.54 per 1,000 admissions in 2009 . Overall, $19.2 \%$ of all BSIs were primary BSI and other important sources included skin/soft tissues (29.3\%), and pneumonia (11.8\%). The most common MRSA strains by PFGE were CMRSA-2 (Ridom spa type t002) with 54.1\%, and CMRSA-10 (Ridom spa type t008) with $29.3 \%$ of the 518 isolates available. Most (63.1\%) community-associated (CA) clones were from patients in western Canada. CMRSA10, traditionally recognised as CA strain was equally isolated from patients whose infections were clinically defined as healthcare-associated. No isolates with reduced susceptibility to vancomycin were identified. Mortality was associated $(P<0.001)$ with older age (OR 2.3) and respiratory site as source of infection (OR 3.0).

CONCLUSION: MRSA BSIs were associated with 24\% 30-day mortality. One-third of BSIs were caused by CA strains (CMRSA 10/7). Certain host factors (age and infection site) were associated with increased mortality.

\section{P35}

SURVEILLANCE FOR COMMUNITY ACQUIRED METHICILLIN-RESISTANT STAPHYLOCOCCUS AUREUS (CA-MRSA) STRAINS ISOLATED FROM BLOOD CULTURES IN QUÉBEC

\section{$\underline{S}$ LÉVESQUE $^{1}$, B LEFEBVRE ${ }^{1}$, L-A GALARNEAU ${ }^{2}$, A-M BOURGAULT $^{1}$}

${ }^{1}$ Laboratoire de santé publique du Québec/Institut national de santé publique du Québec, Sainte-Anne-de-Bellevue;

${ }^{2}$ Centre hospitalier de Trois-Rivières, Trois-Rivières, QC

OBJECTIVE: To determine the proportion of MRSA bacteremias caused by CA-MRSA strains in the province of Québec.

METHODS: MRSA blood culture strains isolated from 2009-04 to 2010-03 were analyzed. Patient's information was collected through a standardized questionnaire. The detection of nuc, mecA and PVL toxin genes was done by PCR and molecular typing by PFGE. The susceptibility profile was determined by a microbroth dilution technique.

RESULTS: A total of 270 strains were characterized: $70.7 \%$ of the isolates were healthcare-associated (HCA). All strains were susceptible to daptomycin, doxycyclin, gentamicin, TMP-SMX and vancomycin; $99.6 \%$ were resistant to levofloxacin, $95.2 \%$ to erythromycin and $85.9 \%$ to clindamycin (CLI). Two strains were resistant to rifampicin and one to linezolid. There were 33 strains with the CMRSA-10 pattern: 32 of those carried the PVL toxin gene and 27 were susceptible to CLI. These strains were responsible for 26 community acquired and 7 HCA bacteremias. Amongst the other 237 strains, 198 were CMRSA-2, 1 CMRSA- 8 and 38 different from the ten known CMRSA profiles. Only 11 stains of other PFGE patterns were susceptible to CLI. The sensitivity, specificity, positive and negative predictive values of CLI susceptibility as a predicting factor for CMRSA-10 were $81.8 \%, 95.4 \%, 71.1 \%$ and $97.4 \%$, respectively.

CONCLUSION: CA-MRSA strains were responsible for $12.2 \%$ of all MRSA bacteremias and 3.7\% of HCA bacteremias. CLI susceptibility and the presence of PVL toxin gene are acceptable markers for CA-MRSA.

\section{P36}

VALIDATION OF ETEST GRADIENT STRIPS FOR DAPTOMYCIN (DAP) MIC DETERMINATION IN METHICILLINRESISTANT AND METHICILLIN-SUSCEPTIBLE STAPHYLOCOCCUS AUREUS AND COAGULASE-NEGATIVE STAPHYLOCOCCI (MRSA, MSSA, CNST)

BM WILLEY ${ }^{1}$, B MINNEMA ${ }^{2}$, P GNANASUNTHARAM ${ }^{1}$, A GELOSIA ${ }^{1}$, N KREISWIRTH ${ }^{1}$, D LOW ${ }^{1,2}$, K WONG $^{1,2}$, SM POUTANEN ${ }^{1}$

${ }^{1}$ Mount Sinai Hospital/University Health Network; ${ }^{2}$ University of Toronto, Toronto, ON

BACKGROUND: DAP Etest (ET) was compared to broth microdilution (BMD) for its ability to determine DAP MIC in staphylococci.

METHODS: Of 364 strains tested, 301 (82\%) were clinical [135 consecutive blood (84 MSSA, 51 MRSA), 67 diverse MRSA, 99 blood CNST (51 S. lugdunensis, 28 S. epidermidis, 20 other)] and 63 controls [19 Cubist MRSA DAP non-susceptible (NS MIC 2-8 mg/L), 44 NARSA VISA (38 MR, $6 \mathrm{MS})$ ]. Using the same inoculum, DAP ET (BD MHA Ca ${ }^{++}$ $25 \mathrm{mg} / \mathrm{L}$ ) was done in parallel to CLSI BMD (Difco MHB DAP Ca ${ }^{++}$ $50 \mathrm{mg} / \mathrm{L})$. Concordant $18 \mathrm{~h}$ ET MIC from 5 readers were used.

RESULTS: Compared to BMD, ET categorical agreement was 95.9\% (95\% CI 93.3-97.5), essential agreement was 85.7\% (95\% CI 81.7-89), major error (ME) rates were 4.8\% (95\% CI 2.9-7.8); and very major error (VME) rates were $0 \%$ (95\% CI 0-8). Notably, ET MIC were $\geq 1$ dil. higher than BMD in 63.7\% strains (95\% CI 58.7-68.5) ( $\chi^{2}$ for trend: 57.292, $\mathrm{d} \mathrm{f}=1, \mathrm{P}<0.0001$ ) (Fig.).

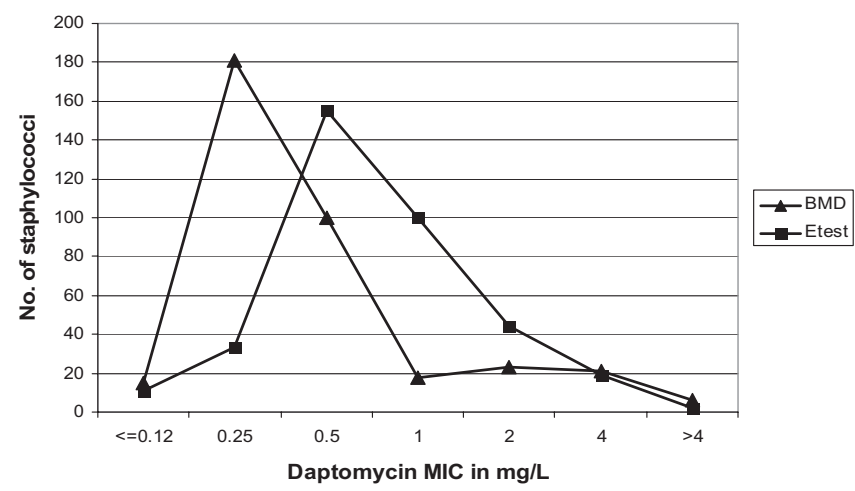

CONCLUSIONS: As ET overestimated DAP MIC by $\geq 1$ dil. and ME exceeded Cumitech 31A's $\leq 3 \%$ limit, all DAP ET NS results should be confirmed by BMD.

\section{P37}

THE INCIDENCE OF MULTI-DRUG RESISTANCE ACINETOBACTER BUMANNII AND ITS INVOLVEMENT IN HOSPITAL INFECTIONS: ONE YEAR PROSPECTIVE STUDY M HALWANI, K AL-HARTHI, NA RASIN

The Infection Control Sterilization and Medical Waste Management Program, Health Affairs, Jeddah, Saudi Arabia

BACKGROUND: Emergence and spread of Acinetobacter bumannii, resistant to most available antimicrobial agents, is an area of great concern. 
OBJECTIVE: To determine the incidence of multi-drug resistance Acinetobacter bumannii from the identified Gram negative bacteria and its involvement in hospital infections.

METHODS: A one year follow-up (January to December 2010) of the clinical isolates of hospitalized patients in twelve different hospitals and the monitoring of the involvement of these isolates in hospital infections. RESULTS: In total, subsequent data from the twelve hospitals revealed that Acinetobacter bumannii counted for 17\% (754/4404) of the identified Gram negative bacteria of which $18 \%$ were involved in different hospital infections. The resistance rate was 25\% Ceftazidime, 26\% Ciprofloxacin, 27\% Cefepime, 34\% Amikacin, 52\% Tazocin, 57\% Gentamicin and 66\% Imipenem. Only $8 \%$ of the isolates were tested for colistin and no resistance was detected.

CONCLUSIONS: There is an urgent need to enforce precise infection control measures and increase in-hospital educational efforts. The application of an antimicrobial stewardship program is inevitable to minimize the spread of these problematic resistant bacteria. Moreover, molecular typing is also needed to identify the responsible clone(s) and determine the possible modes of transmission.

\section{P38}

SURVEILLANCE OF GRAM-NEGATIVE INTRA-ABDOMINAL AND URINARY TRACT PATHOGENS IN EDMONTON, ALBERTA, 2008-2010: THE SMART STUDY

R RENNIE ${ }^{1}$, LA TURNBULL ${ }^{1}$, A JOHNSON ${ }^{2}$

${ }^{1}$ University of Alberta Hospital, Edmonton, $\mathrm{AB}$;

${ }^{2}$ IHMA, Schaumburg, USA

OBJECTIVE: SMART (Study for Monitoring Antimicrobial Resistance Trends) is a global surveillance program that focuses on infections for which Ertapenem is indicated. The purpose of SMART is to monitor susceptibility of these organisms to a variety of antimicrobial agents, and to monitor the before and after use impact of Ertapenem. 324 gram-negative strains from the University of Alberta Hospital were examined between 2008 and 2010.

METHODS: A total of 324 gram-negative clinical isolates of intraabdominal and urinary tract infection pathogens were submitted from our institution to SMART. The antimicrobial susceptibility of these isolates was determined by CLSI broth microdilution methodology. Antimicrobials monitored were ampicillin-sulbactam (AS), amikacin (AK), ceftriaxone (CAX), ceftazidime (CAZ), cefotaxime (CFT), cefoxitin (CFX), ciprofloxacin (CP), cefepime (CPE), Ertapenem (ETP), imipenem (IMP), levofloxacin (LVX) and pipercillin/tazobactam (P/T). Results are entered into a website portal - the Surveillance Data Link Network (SDLN ${ }^{\mathrm{TM}}$ ) which is available to the investigator and to other members of the SMART surveillance program for comparison.

RESULTS: Percent susceptibility results for all organisms combined are: A/S (54), AK (99), CAX (85), CAZ (90), CFT (86), CFX (76), CP (80), CPE (96), ETP (96), IMP (94), LVX (82) and P/T (92). Overall, both IMP and ETP were highly effective against these gram-negative microorganisms. There was considerably greater resistance to LVX (18\%) and CP (20\%). CPE and P/T remain highly effective agents. We did not observe any major changes in susceptibility of these organisms to the carbapenems during the surveillance period.

CONCLUSIONS: Laboratory-based antimicrobial surveillance is designed to detect shifts in resistance to specific agents over time. We did not observe any changes in susceptibility to the carbapenems, imipenem and ertapenem during this surveillance period. Imipenem is widely used in our institution, but is used for specific indications in serious infections. There was no effect noted on ertapenem resistance in this study.

\section{P39}

TWO CASES OF SIGNIFICANT ADVERSE PATIENT EVENTS DUE TO CONTACT ISOLATION PRECAUTIONS IN AMBULATORY CARE PROGRAMS - TIME TO REASSESS EVIDENCE AND POLICIES

L SAXINGER, G TAYLOR

University of Alberta, Edmonton, $A B$

BACKGROUND: Contact isolation practices are effective in limiting spread of methicillin resistant $S$. aureus (MRSA) within the hospital environment, but emerging literature documents adverse consequences of isolation practices, including less patient-health care worker contact, changes in systems of care with more noninfectious adverse events, increased patient depression and anxiety, and decreased patient satisfaction. In ambulatory settings there is no demonstrated benefit of isolation practices for MRSA, with little or no literature addressing its adverse consequences. We report 2 MRSA colonized patients with significant adverse consequences due to isolation practices in ambulatory care. (Demographic details may be changed to protect identity.)

CASE 1: A 68 year old man developed a wound infection after a rectosigmoid resection for adenocarcinoma; MRSA grew in culture. The infection resolved with antimicrobial therapy, but 1 year later he remained MRSA colonized on nasal culture. He was referred to a hospital based education program for cancer patients, but when his MRSA status was noted, was excluded from participation, "according to hospital policy". The patient expressed feelings of severe stigmatization and shame over these events.

CASE 2: A 42 year old male with known MRSA colonization, HIV infection, substance abuse and history of homelessness was established on successful antiretroviral therapy. He was very engaged in improving his life and enrolled in a residential drug rehabilitation program. The program policy required him to wear a gown, gloves and mask while attending group programs, and in common spaces. The patient was then stigmatized and physically threatened by other clients in the program. In fear for his safety, he discharged himself from the rehabilitation program, and subsequently he stopped taking his HIV medications, resumed his substance abuse and again became homeless.

CONCLUSION: Health care workers treating MRSA colonized patients in ambulatory programs should adhere to Routine Practices, and counsel patients on hygiene. Contact isolation precautions create the potential for significant, life altering adverse events related to stigmatization and exclusion, in the absence of documented benefit. These policies should be reviewed.

\section{P40}

\section{EFFICACY OF ALCOHOL-BASED HAND HYGIENE ON SPORE-FORMING BACTERIA IN A COMMUNITY HOSPITAL INTENSIVE CARE UNIT}

M KELLY, M DELORME, W DANDJINOU

Hôpital Jean Talon, Montreal, QC

OBJECTIVE: To measure the performance of alcohol-based hand hygiene used in compliance to WHO guidelines on an intensive care unit.

METHODS: During a 6 hour observation period, the hands of six healthcare workers were plated on sheep-blood agar at ten specific times: At the beginning of their working shift; before and after using an alcohol-based hand-rub (MicroSan) immediately after having cared for a patient on four occasions, 1 hour apart; before leaving for their lunch break. The plates were incubated at $37^{\circ} \mathrm{C}$ in aerobic conditions for 48 hours and bacterial CFUs were counted according to the three following categories: Micrococcaceae, Bacillus sp, other. Efficacy was calculated by comparing the number of CFUs in each category before and after each patient care episode.

RESULTS: Micrococcaceae represented 53\% and Bacillus 36\% of the total CFUs on the bacterial plates. The alcohol-based handrub reduced total CFU counts by $72 \%$ globally: $96 \%$ for Micrococcaceae, $16 \%$ for Bacillus sp. When compared to the initial plates, the final plates showed a decrease of $51 \%$ of the Micrococcaceae count but a $125 \%$ increase in the Bacillus sp. CFU counts.

CONCLUSION: The alcohol-based hand rub demonstrated high efficacy in reducing the density of aerobic Gram positive cocci on the hands of 
health care workers. However, its efficacy on sporulated bacteria was six times lower. The results also suggest that there was selection and accumulation of sporulated bacteria during the observation period.

\section{P41}

HEY BABY WHAT'S YOUR SIGN? EDUCATIONAL INFECTION CONTROL SIGNS IN A CANADIAN PEDIATRIC ACUTE CARE HOSPITAL

\section{S FORGIE $^{1,2}$, J DURAND ${ }^{1}$, S GILBRIDE ${ }^{1}$, S WELLING ${ }^{1}$}

${ }^{1}$ Stollery Children's Hospital; ${ }^{2}$ University of Alberta, Edmonton, AB OBJECTIVES/BACKGROUND: Children admitted to acute care facilities with a viral respiratory infection should be placed on contact and droplet precautions. Our previous isolation signs were created for adult units, and two signs were required - increasing the chance for error. A new pediatric sign that incorporated both contact and droplet precautions was piloted.

METHOD: Signs with a stick figure, explanations and overlay of personal protective equipment (PPE) were created to educate healthcare workers. A hospital wide contest was held to name the stickperson and the new signs were piloted on one unit. A questionnaire was distributed, changes were made to the signs and they were then distributed hospital wide.

RESULTS: 225 staff members contributed suggestions for the name of the stick figure. The winning entry was "Captain Germnomore" and the contest generated a lot of discussions. 50 questionnaires were sent out with a $56 \%$ response rate. All respondents were female and the majority $(57 \%)$ were $20-30$ years of age. $86 \%$ were nurses, $7 \%$ student nurses and $7 \%$ allied healthcare workers. All noticed the new signs, and $54 \%$ liked the overlay of PPE on the stick figure the most. $89 \%$ found these signs more noticeable than the older signs. $68 \%$ felt the new signs increased their understanding of required infection control procedures outlined on them. $93 \%$ wanted similar signs for other isolation precautions. Comments were positive: "All the signs should be like this," "Easy to understand," "You only need 1 sign instead of 2," "User friendly."

CONCLUSIONS: Implementation of new signs promoted understanding of infection control procedures.

\section{P42}

PERSONAL PROTECTIVE EQUIPMENT USE AMONG CANADIAN HEALTHCARE WORKERS DURING THE DELIVERY OF CARE TO PATIENTS ON DROPLET PRECAUTIONS

R MITCHELL ${ }^{1}$, V ROTH ${ }^{2}$, G ASTRAKIANAKIS ${ }^{3}$, E BRYCE ${ }^{4}$, R GERVAIS $^{1}$, D GRAVEL ${ }^{1}$, L JOHNSTON ${ }^{5}$, G TAYLOR ${ }^{6}$, $M_{\text {VEARNCOMBE}}{ }^{7}$, K WILKINSON ${ }^{1}$; Canadian Nosocomial Infection Surveillance Program ${ }^{1}$

${ }^{1}$ Centre for Communicable Diseases and Infection Control, Public Health Agency of Canada; ${ }^{2}$ The Ottawa Hospital, Ottawa, ON; ${ }^{3}$ University of British Columbia; ${ }^{4}$ Vancouver General Hospital, Vancouver, BC; ${ }^{5}$ Queen Elizabeth II Health Sciences Centre, Halifax, NS; ${ }^{6}$ University of Alberta Hospital, Edmonton, AB; ${ }^{7}$ Sunnybrook Health Sciences Centre, Toronto, ON OBJECTIVES: To determine the proportion of healthcare workers (HCWs) appropriately selecting, wearing, and removing PPE for droplet precautions during the second wave of the influenza A H1N1 pandemic in Canadian hospitals.

METHODS: An observational study was performed in seven hospitals in Ontario and Manitoba between February 9, 2010 and April 8, 2010. Using a standardized data collection tool trained observers recorded HCWs selecting, putting on and taking off PPE during the delivery of care to inpatients on droplet precautions. A composite score was derived for correct PPE use as determined by hospital policy.

RESULTS: Observations of $204 \mathrm{HCW}$ s were recorded of which 110 were complete. Only six HCWs (6\%) applied all components of proper PPE use. There were $29 \%$ of HCWs who selected the appropriate PPE, $76 \%$ who put on PPE correctly, $72 \%$ who removed PPE using the correct procedure, and $29 \%$ who removed PPE in the correct sequence. A significantly higher proportion of nurses than other clinical HCWs removed PPE correctly
( $81 \%$ vs. $64 \% ; \mathrm{p}=0.04$ ). As well, significant differences in PPE use between units across all facilities were observed.

CONCLUSIONS: HCWs were observed to perform poorly in applying all components of proper PPE use (selection, putting on, and removing). Reasons for this poor performance should be explored to be better prepared for management of patients on droplet precautions, including during future pandemics.

\section{P43}

SEROCONVERSION AFTER ONE DOSE OF ADJUVANTED PH1N1 INFLUENZA VACCINE IN SOLID ORGAN TRANSPLANTED (SOT) PATIENTS

M RESENDE ${ }^{1,2}, \mathrm{~S}^{\text {HUSAIN }}{ }^{1}, \mathrm{C}^{\text {ROTSTEIN }}{ }^{1}$

${ }^{1}$ University of Toronto, Toronto, ON; ${ }^{2}$ University of Campinas, Campinas, Brazil

BACKGROUND: SOT patients are more highly susceptible to $\mathrm{pH} 1 \mathrm{~N} 1$. As a result, they were a target group for $\mathrm{pH} 1 \mathrm{~N} 1$ vaccination. However few studies have evaluated the immunogenicity of the vaccine in this group. During 2009, due to influenza pandemic H1N1 (pH1N1), one dose of adjuvanted H1N1 pandemic influenza vaccine (Arepanrix ${ }^{\mathrm{TM}}$, GlaxoSmithKline Canada) was employed.

OBJECTIVE: To evaluate the seroconversion after one dose of adjuvanted H1N1 pandemic influenza vaccine in SOT patients.

METHODS: We enrolled 5 adults (age $>18$ y) SOT patients submitted (1 liver, 2 kidney and 2 lung transplants) from the Multi-organ Transplant Program at the University Health Network to receive one dose of $3.75 \mathrm{mcg}$ adjuvanted pHIN1 vaccine. Serologic status was evaluated through haemagglutination inhibition (HI) assay before and two and 4 weeks post-vaccination. Vaccine related-seroconversion was defined as post-vaccination titre of $\geq 1: 40$ for subjects who were seronegative prevaccination or a 4-fold increase in post-vaccination compared to prevaccination titre.

RESULTS: The median age of patients was 50 years, and 3 were male. The median time post-transplant was 3 years (range 2 months to 15 years). All patients were on a double or triple immunosuppressive regimen. Prevaccination $\mathrm{pH} 1 \mathrm{~N} 1$ titre was 1:10 in 4 patients and 1:40 in one lung transplant patient. Seroconversion and seroprotection were observed only in the latter case, from 1:40 at baseline to 1:320 at both two and four weeks after vaccination. In this case the lung transplant was done 34 months before vaccination and the immunosuppressive regimen was tacrolimus, azathioprine and prednisone. Another lung transplant patient, without seroconversion to $\mathrm{pH} 1 \mathrm{~N} 1$, had the same titre to Brisbane H1N1 (1:40) at baseline, as well as two and four weeks post-vaccination.

CONCLUSION: This small sample size calls into question whether one dose of adjuvanted $\mathrm{pH} 1 \mathrm{~N} 1$ vaccine did in fact provide seroprotection in SOT patients. It may have been that one dose was inadequate, perhaps seroconversion would have taken longer or that the $\mathrm{HI}$ assay did not measure seroprotection adequately. These results may drive the necessity to evaluate the immune response to other adjuvanted influenza vaccines in a larger number of patients and if additional doses can improve the seroprotection rate.

\section{P44}

PRODUCTION OF STANDARDIZED CONTROL SAMPLES FOR ASSESSING INTRA- AND INTER-LABORATORY VARIABILITY OF INFLUENZA RT-PCR DETECTION TESTS DURING THE 2009 A(H1N1) INFLUENZA (PH1N1) PANDEMIC

H CHAREST, É BÉLANGER-TRUDELLE, M LORTIE, M FAUVEL, M COUILLARD

Laboratoire de santé publique du Québec/INSPQ,

Sainte-Anne-de-Bellevue, QC

BACKGROUND: In the fall of 2009, real time RT-PCR ( $r$ tRT-PCR) assays to detect $\mathrm{pH} 1 \mathrm{~N} 1$ were decentralized to 9 hospital laboratories in the Province.

OBJECTIVES: 1) Provide sufficient stocks of standardized control samples to all laboratories offering $\mathrm{pH} 1 \mathrm{~N} 1$ tests; 2 ) evaluate the stability 
of control stock reagents; 3) assess variability in pH1N1 rtRT-PCR assays.

METHODS: Positive controls were prepared using a heat inactivated $\left(56^{\circ} \mathrm{C}\right.$ for $\left.30 \mathrm{~min}\right)$ isolate of $\mathrm{pH} 1 \mathrm{~N} 1$ cultured on MDCK cells and diluted with culture medium (MEM) to obtain a pH1N1 $r$ RT-PCR Ct of 30, a value close to 10 -fold the limit of detection using a $r t$ RT-PCR pH1N1 assay. Negative control stocks consisted of MEM only. For each control, more than 5,000 aliquots $(0.25 \mathrm{ml})$ were prepared in a single batch and shipped on dry ice to hospitals. Laboratories were instructed to thaw aliquots only once, to use 1 set of controls per series of 22 samples or less, to compile Ct values obtained for control samples and to submit their results together with methods and kit lot numbers. $r$ RTT-PCR Ct values for influenza $\mathrm{A}$ and $\mathrm{pH} 1 \mathrm{~N} 1$ subtype tests were analyzed using Levey-Jennings plots.

RESULTS: Five laboratories, using 3 different $r$ RTT-PCR assays, submitted a total of 680 data point sets over a 5 month period. The inter-laboratory mean $\mathrm{Ct}$ values ranged between 28.6 and 34.0. The intra-laboratory reproducibility measurements varied from 0.7 to $3.4 \mathrm{Ct}$ units ( 2 standard deviations). No false positive results were reported. One laboratory experienced a failure rate $>5 \%$ with positive controls. The monitoring over time showed no significant variation in Ct value trends, suggesting that control reagents remained stable for up to 5 months.

CONCLUSIONS: The centralized mass production of cell culture derived control samples for $r t$ RT-PCR assays is simple, and allows the monitoring of intra- and inter-laboratory performance over time.

\section{P45}

RESPIRATORY INFECTIONS IN INSTITUTIONS DURING THE SECOND WAVE OF PANDEMIC (H1N1) 2009

A MARCHAND-AUSTIN ${ }^{1}$, N LOMBARDI ${ }^{2}$, A ESHAGHI ${ }^{2}$, E LOMBOS $^{2}$, D ALEXANDER ${ }^{2,3}$, S PATEL ${ }^{2,3}$, J LONGTIN ${ }^{2}$, E KRISTJANSON ${ }^{2}$, F JAMIESON ${ }^{2,3}$, D LOW ${ }^{2,3,4}$, J GUBBAY, 2,3

${ }^{1}$ Public Health Agency of Canada, Winnipeg, MB; ${ }^{2}$ Ontario Agency for Health Promotion and Protection; ${ }^{3}$ University of Toronto;

${ }^{4}$ Mount Sinai Hospital, Toronto, ON

OBJECTIVES: At the onset of Pandemic (H1N1) 2009 (pH1N1) in April 2009, enhanced surveillance of respiratory infections in institutions was instituted. pH1N1 was only identified in 2 of 83 outbreaks tested during the first wave between 20 April and 12 June 2009. Laboratory data from 13 June to 30 November 2009 was reviewed to document circulating respiratory viruses in the period preceding and during the second wave of intense $\mathrm{pH} 1 \mathrm{~N} 1$ activity.

METHODS: Specimens from respiratory institutional outbreaks were tested by real-time reverse transcription-PCR (rRT-PCR) for influenza A virus matrix gene. Matrix gene positives were then subtyped for pH1N1. Multiplex molecular respiratory virus panels were also used.

RESULTS: Entero/rhinovirus was detected in $214(60 \%)$ and pH1N1 in 77 (22\%) of 354 outbreaks tested. Long Term Care Facilities (LTCF) accounted for $250(71 \%)$ of the outbreaks tested but only 19 (25\%) of pH1N1 positive outbreaks. Conversely, schools accounted for only $37(10 \%)$ of the outbreaks tested but $37(44 \%)$ of $\mathrm{pH} 1 \mathrm{~N} 1$ positive outbreaks. Entero/rhinovirus was detected in 169 (68\%) outbreaks from LTCFs in contrast to pH1N1 being detected in 19 (7.6\%).

CONCLUSIONS: pH1N1 was only responsible for a small proportion of LTCF outbreaks in the second pandemic wave. Enhanced surveillance using molecular methods provided valuable insight into the significant impact of entero/rhinovirus in the midst of a pandemic. Such information is key for the use of empiric antiviral therapy in respiratory outbreaks during periods of increased influenza activity, including pandemics.

\section{P46}

MULTIDRUG-RESISTANT PANDEMIC INFLUENZA A (H1N1) INFECTION IN AN IMMUNOCOMPETENT AMBULATORY CHILD WITH NO PRIOR ANTIVIRAL EXPOSURE

A ESHAGHI ${ }^{1}$, S PATEL ${ }^{1,2}$, A SARABIA ${ }^{3}$, R HIGGINS ${ }^{1}$, A SAVCHENKO $^{2}$, P STOJIOS ${ }^{2}$, Y LI $^{4}$, N BASTIEN ${ }^{4}$, D ALEXANDER ${ }^{1,2}$, D LOW ${ }^{1,2,5}$, L GUBBAY ${ }^{1,2,5,6}$

${ }^{1}$ Ontario Agency for Health Protection and Promotion; ${ }^{2}$ University of Toronto, Toronto; ${ }^{3}$ The Credit Valley Hospital, Mississauga, ON; ${ }^{4}$ Public Health Agency of Canada, Winnipeg, MB; ${ }^{5}$ Mount Sinai Hospital; ${ }^{6}$ The Hospital for Sick Children, Toronto, ON

OBJECTIVES: Recent case reports describe multidrug-resistant pandemic influenza A (H1N1) 2009 infection in immunocompromised patients exposed to neuraminidase inhibitors due to an I223R neuraminidase mutation. We report the first case of multidrug-resistant H1N1 2009 bearing the I223R mutation in an ambulatory child with no previous exposure to neuraminidase inhibitors.

METHODS: A 15 year old girl with a history of asthma sought treatment for influenza like illness at an emergency room. H1N1 2009 was detected by real-time RT-PCR. The specimen was cultured in rhesus monkey kidney cells and whole genome sequencing was performed using a modified WHO protocol.

RESULTS: Compared to A/California/7/2009 (H1N1), several nonsynonymous mutations were identified, including I223R in neuraminidase. Compared to wild type control, the I223R mutant exhibited 69.1 and 10.7 fold increases in $\mathrm{IC}_{50}$ s for oseltamivir and zanamivir, respectively.

CONCLUSIONS: Clinical significance of the I223R mutation is poorly understood, since $\mathrm{IC}_{50}$ s for oseltamivir and zanamivir are well below achievable levels in serum. Our case is unique as she had no prior exposure to NAIs, was immunocompetent, and had an uneventful recovery. This first report of community acquisition of a multidrug-resistant strain of H1N1 2009 reinforces the need to continue close monitoring for the emergence of resistant viruses and incorporation of screening for newly discovered resistance mutations into clinical diagnostics.

\section{P47}

MODELLING OF LABORATORY TESTING FOR AN INFLUENZA PANDEMIC: EVALUATION OF A MODEL USING REAL-TIME DATA FOR ONTARIO FROM THE SECOND WAVE 2009 H1N1 PANDEMIC

K MINNINGS $^{1}$, A KOU ${ }^{1}$, G EVANS ${ }^{1,2}$, T DAY ${ }^{1}$, A MAJURY ${ }^{1,2}$

${ }^{1}$ Queen's University; ${ }^{2}$ Ontario Agency for Health Protection and Promotion, Kingston, ON

OBJECTIVE: The purpose of the study was to predict the number of influenza laboratory tests anticipated in the event of a pandemic, using modelling, and to use this information to optimize the laboratory response. The model developed was then validated against the laboratory findings from the second wave pH1N1.

METHODS: The parameters that the model used are: Attack rate: 18\%, Average infectiousness: 2 days, Maximum duration of infectiousness: 3 days.

RESULTS: The model predicted the number of specimens submitted (smooth curve) and the actual number of specimens submitted (shaded bars) in Ontario during second wave pH1N1 (top figure). Numbers predicted by the model, were compared to numbers of specimens received and numbers of specimens testing, in Ontario, during the second wave of pH1N1 (bottom figure). 

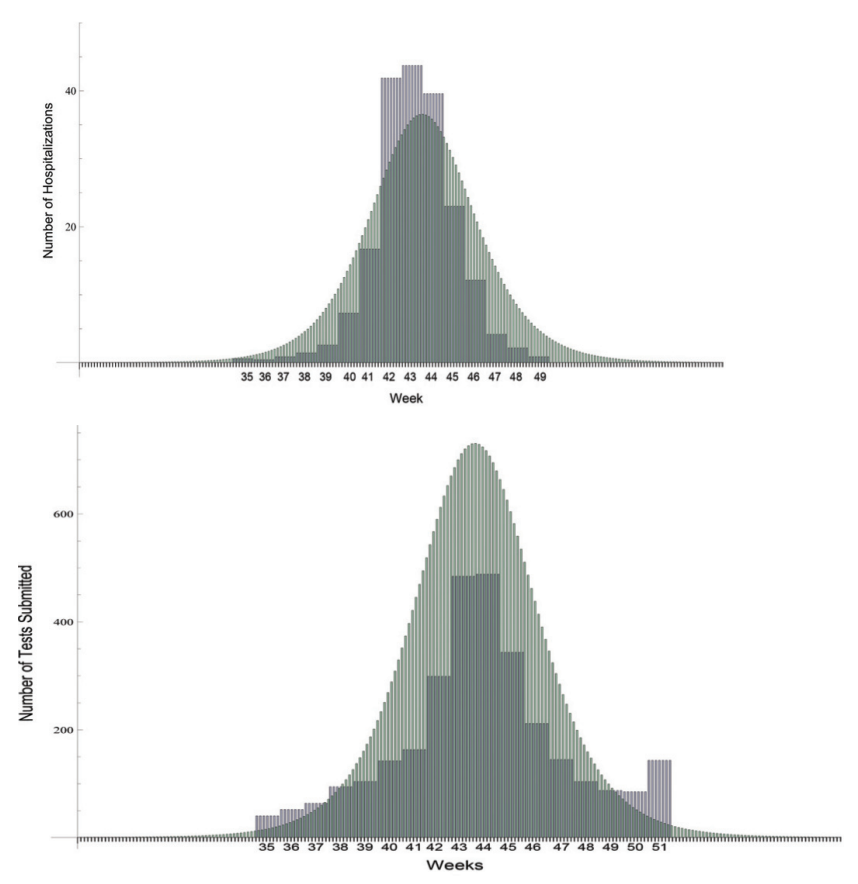

CONCLUSIONS: 12,202 more specimens were predicted than actually submitted. These differences can be explained by gate keeping measures introduced by laboratories and public health agencies at the time of the pandemic. Modelling successfully aided in laboratory pandemic preparation.

\section{P48}

FREQUENT DETECTION OF RHINOVIRUS/ENTEROVIRUS AMONGST CHILDREN HOSPITALIZED WITH RESPIRATORY INFECTION

S MATHUR $^{1}$, J BABWAH ${ }^{2}$, S CHONG $^{2}$, J MAHONY ${ }^{1}$, M SMIEJA $^{1}$

${ }^{1}$ McMaster University; ${ }^{2}$ St Joseph's Healthcare, Hamilton, $\mathrm{ON}$ OBJECTIVE: Influenza and Respiratory Syncytial Virus (RSV) are the primary identified causes of pediatric hospitalizations for respiratory illness. However, with the development of multiplex PCR assays for respiratory virus detection, an important role for rhinovirus and enterovirus is emerging. We studied the frequency of rhinovirus/enterovirus detection amongst pediatric inpatients with respiratory disease.

METHODS: From consecutive nasopharyngeal swabs (NPS) submitted to the Regional Virology Laboratory at St Joseph's Healthcare in Hamilton, Ontario, we obtained a stratified random sample of up to 50 children per month for 12 consecutive months. Viruses were detected by the Luminex Respiratory Virus Panel (RVP version 1, Austin TX). Chart reviews were performed using standardized case report forms detailing medical history, symptoms, admission details, diagnosis and treatment.

RESULTS: A total of 423 children were identified. Of these, 163 tested negative for all viruses. RVP detected 29 cases of influenza A or B, 34 RSV, 9 coronaviruses and 135 for rhino/enterovirus. Rhino/enterovirus patients had a mean age of 2.3 years, a median length of stay of 1 day, (Q1-Q3: 1 , 2 days), and $4.4 \%$ required ICU admission. Length of stay was similar for other respiratory viruses. Rhino/enterovirus patients accounted for 14 of 40 pneumonia diagnoses from the study period.

CONCLUSION: Rhino/enterovirus was the most commonly detected respiratory virus amongst pediatric inpatients, and was associated with a similar length of stay and rate of ICU admission as other respiratory viruses.

\section{P49}

EVALUATION OF THE SEEPLEX ${ }^{\circledR}$ RV12 MULTIPLEX PCR ASSAY FOR THE DETECTION OF COMMON RESPIRATORY VIRUSES

C KERN ${ }^{1}$, L SHAW ${ }^{1}$, L SULLIVAN ${ }^{1}$, I KARNAUCHOW ${ }^{1,2}$

${ }^{1}$ Regional Virology Laboratory, Children's Hospital of Eastern

Ontario; ${ }^{2}$ University of Ottawa, Ottawa, ON

OBJECTIVE: Diagnostic laboratories increasingly rely on molecular detection and identification of respiratory viruses. Seeplex® RV12 ACE Detection (RV12; Seegene Inc., Rockville, MD), a DPO-based multiplex assay, interrogates samples for the presence of 12 viruses by PCR, followed by a choice of detection method. We compared the performance of RV12 plus Lab901 Screentape (Lab901 Ltd., Loanhead, UK) analysis to reference molecular assays for detection of RSV, influenza- (Flu), adeno- (Adv), corona-(CoV), human metapneumo-(hMPV), parainfluenza- (PIV), and rhino- (RV) viruses.

METHODS: Respiratory specimens submitted to the Regional Virology Laboratory (RVL; 04/23/09-10/24/09) from adult and pediatric patients, tested for influenza by realtime PCR (RTPCR) ( $n=170: 82$ FluA positive, $88 \mathrm{FluA} / \mathrm{B}$ negative) were retrospectively tested by RV12. Discordant RV12 Flu results and all non-Flu RV12-positive results were analyzed by in-house-validated molecular assays.

RESULTS: RV12 detected and identified all 82 known FluA (+) specimens ( 12 adult, 70 pediatric): relative to RTPCR, RV12 FluA sensitivity and specificity were $100 \%$. RV12 detected single agent infections by FluA (72), RV (10), PIV3 (4), CoV (3), hMPV (2), AdV (1), and RSV (1). Twelve co-infections were identified: FluA plus: RV (5), AdV (2), hMPV (1), and PIV3 (1); RV plus: AdV/PIV3 (1) and AdV (1), and RSV/RV/ PIV3 (1). All RV12 detections were confirmed by reference PCR, save for PIV3. 5 of 7 PIV3 (+) RV12 results could not be confirmed. Target-specific RV12 performance data will also be presented.

CONCLUSION: Seeplex ${ }^{\circledR}$ RV12 / Lab901 displayed excellent sensitivity and specificity for FluA (H1N1)-2009. It also accurately detected and identified several infections/co-infections caused by other viruses, with sensitivity similar to that of reference PCR assays. RV12 /Lab901 did overcall PIV3 infections (71\%). With this caveat, the Seeplex ${ }^{\circledR}$ RV12/Lab 901 combination is convenient to use, and is a serious contender in the expanding field of multiplexed pathogen identification systems.

\section{P50}

DEVELOPMENT OF A MULTIPLEX RESPIRATORY VIRUS REAL TIME RT-PCR DESIGNED TO REPLACE TRADITIONAL DETECTION METHODS IN A REGIONAL VIROLOGY LABORATORY

C RUTHERFORD $^{1,2}$, SE DALE ${ }^{2,3}$, D JOHNSON ${ }^{1,2}$, M MACPHERSON ${ }^{1,2}$

${ }^{1}$ St. Joseph's Healthcare; ${ }^{2}$ Hamilton Regional Lab Medicine Program; ${ }^{3}$ McMaster University, Hamilton, ON

BACKGROUND AND OBJECTIVE: The direct fluorescent antibody (DFA) technique and shell vial culture (SVC) are traditional methods used in Virology to detect respiratory viruses. Both techniques are labour intensive and require a high degree of technologist training to perform. While DFA is relatively quick ( 1.5 hours), SVC requires 48 hours and costs may be prohibitive. A real-time RT-PCR was developed to replace DFA/SVC with the aim of increasing sensitivity, improving workflow and reducing costs.

METHODS: Viral nucleic acids were extracted from nasopharyngeal swabs (NPS) in Universal Transport media. The assay detects influenza A and $\mathrm{B}$, respiratory syncytial virus (RSV), human metapneumovirus (hMPV), parainfluenza 1,2 and 3 and adenovirus using the Qiagen Multiplex RT-PCR Probe kit and the Corbett RotorGene 6000. The RNA phage MS2 is included as an internal extraction and amplification control.

RESULTS AND CONCLUSION: In 2009-10 we compared the performance of the PCR using DFA \pm SVC as a gold standard for 1238 specimens. Overall sensitivity/specificity vs DFA ( $n=938$ ) was 100/96.8\% and vs SVC ( $\mathrm{n}=300)$ was $99.7 / 99.6 \%$. For influenza A, B and RSV sensitivity was $100 \%$. For parainfluenza, adenovirus and hMPV, sensitivity was $>95 \%$. 
RT-PCR costs $\sim \$ 10.00 \mathrm{CDN}$ per specimen, including extraction, while DFA/SVC costs $\sim \$ 28.00 \mathrm{CDN}$ per specimen. The RT-PCR is now used in the laboratory with a projected cost savings of $\$ 48,000$ for the 2010-11 respiratory season. We conclude that this assay can effectively replace DFA/ SVC without loss of sensitivity or specificity while concomitantly reducing costs.

\section{P51}

\section{COMPARATIVE EVALUATION OF THE LUMINEX XTAG RVP FAST AND THE QIAGEN RESPLEX II V.2 ASSAYS FOR RESPIRATORY VIRUS DETECTION}

\section{J MÉNARD, H CHAREST}

Laboratoire de santé publique du Québec/INSPQ,

Sainte-Anne-de-Bellevue, QC

OBJECTIVES: 1 ) to evaluate the sensitivity of the Luminex xTAG RVP Fast assay for influenza (Flu) virus detection and 2) to compare results obtained by xTAG RVP Fast and the QIAGEN Resplex II v.2 kits for respiratory viruses in the context of a surveillance program for patients with flu-like illnesses.

METHODS: A total of 137 nucleic acid extracts prepared from nasal flocked swabs and previously analyzed by real time RT-PCR ( $r$ RT-PCR) or by Resplex II were retested using the xTAG RVP kit. In house RT-PCR was used to further investigate discordant results.

RESULTS: The concordance between results is shown in the following table:

\begin{tabular}{lcrrrrrr}
\hline & & \multicolumn{2}{c}{ Objective \#1 } & & \multicolumn{2}{c}{ Objective \#2 } \\
\cline { 3 - 4 } \cline { 6 - 7 } & & \multicolumn{2}{c}{ Flu rtRTPCR } & & \multicolumn{2}{c}{ Resplex II v.2 } \\
& & + & & & & + & - \\
\hline \multirow{2}{*}{ xTAG RVP } & + & 58 & & & 56 & 21 \\
& - & 3 & 71 & & 1 & 27 \\
\hline
\end{tabular}

In addition, 7 samples with $r$ RTT-PCR Ct values $>32$ and negative by Resplex II tested positive for Flu by xTAG RVP. Samples that tested positive for one or more respiratory viruses with both commercial kits included positives for syncytial virus $(n=14)$, parainfluenza virus $(n=9)$, human metapneumovirus (hMPV) $(n=5)$, rhinovirus or enterovirus $(n=8)$, adenovirus $(n=2)$ and coronavirus $(\mathrm{CoV})(\mathrm{n}=4)$. In house RT-PCR for hMPV and CoV confirmed 3 of 4 samples that tested positive by xTAG RVP only.

CONCLUSION: Although both commercial kits generated similar results for the respiratory viruses tested, the xTAG RVP Fast assay was found to be more sensitive than Resplex II v.2 assay for Flu viruses, hMPV and $\mathrm{CoV}$.

\section{P52}

EVALUATION OF COPAN UTM-RT TRANSPORT MEDIUM AND SIGMA VCM MEDIUM FOR THE RECOVERY OF CULTIVATABLE, CLINICAL VIRUS ISOLATES AND COMPARISON OF THE COPAN FLOCKED SWAB WITH THE SIGMA SWAB FOR SPECIMEN COLLECTION

\section{K BRANDT, PN LEVETT, GB HORSMAN}

Saskatchewan Disease Control Laboratory, Regina, SK

OBJECTIVES: (1) To compare the effectiveness of the Copan UTM-RT transport medium and Sigma VCM medium (Medical Wire and Equipment) in preserving the infectivity of influenza A, parainfluenza 3, respiratory syncytial virus (RSV), adenovirus and herpes simplex type 1 (HSV-1); and (2) to compare the Copan flocked swab with the Sigma swab

METHODS: (1) Clinical isolates of each virus were suspended in each transport medium, kept at $4^{\circ} \mathrm{C}$ and $22^{\circ} \mathrm{C}$ and then inoculated into cell cultures on days 2, 4 and 6, simulating transport times of 2, 4 and 6 days respectively. Cell cultures were examined for CPE daily for 6 days, at which time DFA was performed on the cell cultures. (2) Duplicate NP swabs were collected on patients with respiratory illness.

RESULTS: (1) Of the two media, the Sigma VCM medium best preserved influenza $\mathrm{A}$ and parainfluenza type 3 infectivity at both $4^{\circ} \mathrm{C}$ and $22^{\circ} \mathrm{C}$. In contrast, RSV viability declined rapidly in the Sigma VCM medium. Adenovirus and HSV-1 were stable in both media and at both temperatures tested. (2) Equivalent numbers of cells were collected with each type of swab

DISCUSSION: (1) The results indicate that viral transport and subsequent culture isolation are possible using different virus transport media if transport times are kept to a minimum, but if transport extends to longer times or low levels of virus are present in the specimen, infectivity may be compromised. (2) There is no apparent difference between the Copan and Sigma swabs for specimen collection.

CONCLUSIONS: CMRSA7 strains are genetically heterogeneous and this trend may be increasing. Non-toxigenic strains are increasing in frequency, and detection of PVL genes is no longer a reliable marker of CMRSA7 isolation.

\section{ROOM: ST PAUL}

\section{P53}

COMPARISON OF DNA AND RNA HPV DETECTION ASSAYS ON CERVICAL, VAGINAL AND URINE SPECIMENS M CHERNESKY, A LYTWYM, D JANG, M HOWARD, L ELIT, K ONUMA, M KLINGEL, J GILCHRIST, A ECOBICHON-MORRIS McMaster University, Hamilton, ON

OBJECTIVE: To compare APTIMA HPV mRNA and HC2 DNA assays for high risk (HR) HPV detection in PreservCyt Pap (L-Pap) self-collected vaginal swabs (SCVS) and first void urine (FVU).

METHODS: Patients $(n=100)$ signed consent for the collection of 2 vaginal nylon-flocked swabs (Copan) transported in a dry tube or in media (Qiagen). Cervical samples were collected with a cervex brush into PreservCyt vials (Hologic) and FVU was collected. Specimens were tested by APTIMA HPV (Gen-Probe) and HC2 (Qiagen). Discordant samples were tested by Linear Array (LA) (Roche). Patients were considered infected if they were L-Pap positive or had 2 positive non L-Pap specimens.

RESULTS: AHPV was positive in 61 and $\mathrm{HC} 2$ in 59 patients ( 55 in both tests). Specimen and test agreements were strong: both tests on L-Pap (95\%, K.75); wet to dry SCVS using AHPV (89.2\%, K.77), using HC2 (96.0\%, K.90); L-Pap to SCVS ranged between $90 \%$ and $88 \%$; FVU agreement to L-Pap was stronger in AHPV than HC2. AHPV and HC2 showed similar sensitivity and specificity for diagnosis of HPV infections. HC2 showed slightly higher sensitivity and lower specificity for detecting 24 patients with CIN2+ biopsies. LA genotyping explained discordancy between patients.

CONCLUSIONS: AHPV and HC2 assays performed similarly to identify women with DNA or mRNA using L-Pap or SCVS. FVU samples were less effective in identifying infections or CIN2+ precancerous lesions. Dry or wet SCVS may serve as an alternative choice for women avoiding pelvic examination.

\section{P54 \\ VALIDATION OF REAL-TIME RT-PCR FOR MOLECULAR DETECTION OF RUBELLA VIRUS R WEBER, J HIEBERT, A SEVERINI}

National Microbiology Laboratory, Public Health Agency of Canada, Winnipeg, MB

OBJECTIVE: The Rubella virus generally causes a mild childhood disease, however it is of primary concern to women during the first trimester of pregnancy. Risk of spontaneous abortion or Congenital Rubella Syndrome (CRS), which is characterized by severe birth defects, can occur. Although vaccination rates are high in Canada, cases from recent travel or immigration where vaccination is not readily available can still be detected. Screening for the Rubella virus by reverse transcription polymerase chain reaction (RT-PCR) is therefore important in the diagnostic sense as well as for molecular epidemiological purposes. The objective of this project was to complete a full validation of a real time RT-PCR screening assay for detection of the Rubella virus. 
METHODS: Conventional primers specific to the envelope (E1) gene target currently used in diagnostics were adapted to a real-time SYBR Green based RT-PCR followed by an in-run melt analysis of the product. Synthetic RNAs were used to establish the copy number limit of detection for the rubella M33 RNA swab/urine samples. To test the reproducibility and repeatability, dilutions were tested in triplicate for a course of six days. Clinical and panel specimens were also tested to assess specificity, sensitivity and cross reactivity.

RESULTS: The detection limit for the assay was determined to be 80 copies of RNA for the rubella M33 swab/urine dilution producing positive result of $>95 \%$ of runs. Repeatability and reproducibility estimates were both found to be $>95$. The estimated cross-reactivity rate is $<5 \%$.

CONCLUSION: The real-time SYBR Green based RT-PCR has proven to be an efficient, sensitive and specific assay. The in-run melt analysis is advantageous to differentiate between true and non-specific amplification of the E1 gene within the Rubella virus genome.

\section{P55}

MEASLES OUTBREAK POST-OLYMPICS-2010

K GUNADASA $^{1}$, A MAK ${ }^{1}$, P TANG ${ }^{1}$, J BEIRNES ${ }^{2}$, G TIPPLES ${ }^{2}$, M KRAJDEN ${ }^{1}$, M PETRIC $^{1}$, Y CHANG 1

${ }^{1} \mathrm{BC}$ Centre for Disease Control, Vancouver, BC; ${ }^{2}$ National Microbiology Laboratory, Winnipeg, MB

OBJECTIVE: Just after the 2010 winter Olympics, an outbreak of measles occurred in British Columbia. A review of laboratory cases is presented.

METHODS: Measles infection was detected by multiple approaches including: RT-PCR testing of respiratory and urine specimens by the NML which was then implemented at the BCCDC as well as by serology for IgGand IgM-class antibody. RT-PCR positive samples were sequenced to determine the infecting genotype.

RESULTS: A total of 80 blood and respiratory specimens from the outbreak were collected between March 13 and May 28, 2010. Of the 53 respiratory specimens tested by RT-PCR, measles virus RNA was detected in 50 . Of these, 35 were genotype H1, 5 were D8 and of interest 3 were genotype A which likely reflects vaccine administration. A total of 57 sera were reactive for measles IgM antibody; 12 were non-reactive and 8 were equivocal. Of note, RNA was detected in the respiratory specimens from all 57 patients also tested by serology. Of the 18 sera that tested equivocal or non-reactive for IgM antibody to measles, 17 had elevated IgG antibody levels.

CONCLUSIONS: These findings illustrate the dynamics of the IgM and IgG responses compared to detection of the virus by RT-PCR and suggest that measles RNA can be detected in the absence of a measles IgM response and despite the presence of a detectable IgG response. RT-PCR of respiratory samples aides in the diagnosis of acute measles and genotyping of positive specimens can be used to determine the origin of the outbreak virus.

\section{P56}

\section{A REVIEW OF LABORATORY TESTING DURING A MUMPS OUTBREAK}

L GUBBAY $^{1,2}$, K OGBULAFOR ${ }^{1}$, E LOMBOS ${ }^{1}$, R HIGGINS ${ }^{1}$,

A ESHAGHI ${ }^{1}$, D ALEXANDER ${ }^{1,2}$, E KRISTJANSON ${ }^{1}$, S RACEY ${ }^{1}$, J MAREGMEN ${ }^{1}$, G LIM ${ }^{1}$, S PATEL ${ }^{1,2}$, G TIPPLES ${ }^{3}$, D LOW ${ }^{1,2,4}$, S DEEKS ${ }^{1,2}$, T MAZZULLI ${ }^{1,2,4}$

${ }^{1}$ Ontario Agency for Health Protection and Promotion; ${ }^{2}$ University of Toronto, Toronto, ON; ${ }^{3}$ National Microbiology Laboratory, Winnipeg, MB; ${ }^{4}$ Mount Sinai Hospital, Toronto, ON

OBJECTIVE: To compare mumps testing modalities used during a mumps outbreak that began in September, 2009.

METHODS: Testing modalities for mumps, included serology, viral culture and realtime reverse-transcriptase PCR (rRTPCR). Whole genome sequencing (WGS) was performed on one viral culture isolate.

RESULTS: 372 samples were received for culture and rRT-PCR. Buccal specimens had the highest positivity. WGS showed $91 \%$ amino acid correlation between the haemaglutinin-neuraminidase (HN) gene of a mumps isolate and the Jeryl Lynn vaccine strain.

CONCLUSIONS: Buccal swabs were more likely to be rRT-PCR positive than urine rRT-PCR. Further work is needed to better understand the poor match of the HN gene of the outbreak strain with that of the Jeryl Lynn vaccine strain and its implications for vaccine effectiveness.

\begin{tabular}{|c|c|c|}
\hline Test & Samples positive/tested (\% pos) & Persons positive/tested (\% pos) \\
\hline $\begin{array}{l}\text { ggM serology } \\
\text { IgG serology pairs }\end{array}$ & $49 / 853(5 \%)$ & $\begin{array}{l}35 / 698(5 \%) \\
23 / 90(26 \%) \text { seroconverted }\end{array}$ \\
\hline All viral culture & $47 / 372(15 \%)$ & $39 / 255(15 \%)$ patients positive \\
\hline All rRT-PCR & $62 / 372(17 \%)$ & 47/255 (18\%) patients positive \\
\hline rRT-PCR by collection site: & & \\
\hline Buccal & $33 / 158(21 \%)$ & \\
\hline Urine & $19 / 146(14 \%)$ & \\
\hline Oral/Oropharyngeal & $1 / 11(9 \%)$ & \\
\hline Throat/pharyngeal & $3 / 24(13 \%)$ & \\
\hline Nasopharyngeal & $0 / 8$ & \\
\hline CSF rRT-PCR & $1 / 1$ & $1 / 1$ \\
\hline Sputum & $0 / 1$ & \\
\hline Swab - collection site not provided & $5 / 23(22 \%)$ & \\
\hline
\end{tabular}

\section{P57}

EVALUATION AND VERIFICATION OF THE SEEPLEX ${ }^{\circledR}$ MULTIPLEX VIRAL DIARRHOEA PANEL FOR THE SIMULTANEOUS DETECTION OF ROTAVIRUS, ADENOVIRUS, AND NOROVIRUS IN CLINICAL STOOL SPECIMENS R HIGGINS ${ }^{1}$, M CARDONA ${ }^{1}$, S MASNEY ${ }^{1}$, M BENIPRASHAD ${ }^{1}$, A ESHAGHI ${ }^{1}$, LGUBBAY 1,2

${ }^{1}$ The Ontario Agency for Health Protection and Promotion; ${ }^{2}$ University of Toronto, Toronto, ON

OBJECTIVES: Acute viral gastroenteritis is a common disease worldwide with noroviruses and rotaviruses being the leading causes. We describe the verification of the Seeplex ${ }^{\circledR}$ viral diarrhoea assay, a newly developed multiplex reverse transcriptase polymerase chain reaction (RT-PCR) assay for detection of norovirus, rotavirus, adenovirus and astrovirus.

METHODS: 165 clinical specimens were tested. Of these, 145 stool specimens were previously tested for enteric viruses by electron microscopy (EM) and, norovirus I or II by real time RT-PCR. The remaining 20 specimens included other viruses and bacteria. Total nucleic acid was extracted using an automated NucliSenese easyMag (Biomeriuex) and rt-PCR amplification was performed in the Icycler (BioRad). PCR inhibitory effects were assessed by co-amplification of an internal control that is included in the Seeplex ${ }^{\circledR}$ primer mix.

RESULTS: Using EM as a reference in the analysis of rotavirus and adenovirus, or real-time RT-PCR in the analysis of norovirus II and norovirus I, diagnostic sensitivities were: $40 / 40$ (100\%) for rotavirus, 35/36 (97\%) for norovirus II, 19/20 (95\%) for norovirus I and (18/22) 82\% for adenovirus. Specificities were: $100 \%$ for rotavirus, $99 \%$ for both norovirus I and norovirus II and $97 \%$ for adenovirus.

CONCLUSIONS: The Seeplex ${ }^{\circledR}$ multiplex viral diarrhoea assay is sensitive and specific for the simultaneous direct detection of several enteric viral pathogens in stool specimens from patients suffering from gastroenteritis. This multiplex PCR assay also enables identification of viral coinfections.

\section{P58}

A REVIEW OF TEN YEARS OF HEPATITIS C GENOTYPING IN NEW BRUNSWICK: 2000-2009

R GARCEAU, G GIROUARD, L THIBAULT, M MALLET

Hôpital Dr GL Dumont, Moncton, NB

OBJECTIVE: We have been genotyping Hepatitis $\mathrm{C}(\mathrm{HCV})$ for the province of New Brunswick since 1999. We audited our data to determine the ratio of various genotypes $(G)$ and the trends in $G$ over the period 2000-2009.

METHODS: Amplicons were generated with Roche Cobas Amplicor HCV test version 2.0, then genotyped with Inno-lipa HCV assay. Methods were unchanged during the study period. We had access to age, gender and HCV G for statistical analysis. Duplicates were deleted.

RESULTS: We tested 1440 patients. $74.4 \%$ were male. The mean age was 40 years (median: 40.2 , range: $13-89$ ). Table 1 provides distribution of HCV G for the study period in percentage. Nt stands for not further typable.

\begin{tabular}{ccccccccccc} 
1nt & 1a & 1a/b & 1b & 2nt & 2a & 2a/c & 2b & 3nt & 3a & 4 \\
\hline 23.9 & 27.1 & 2.4 & 15.5 & 3.1 & 0.1 & 3.6 & 4 & 0.2 & 19.5 & 0.6 \\
\hline
\end{tabular}

G1 accounted for $68.8 \%$, G2 for $10.8 \%$, G3 for $19.8 \%$ and G4 for $0.6 \%$ of all HCV G. 
Table 2 provides the \% HCV G3 over the total of HCV G per year

\begin{tabular}{llllllllll}
$\mathbf{2 0 0 0}$ & $\mathbf{2 0 0 1}$ & $\mathbf{2 0 0 2}$ & $\mathbf{2 0 0 3}$ & $\mathbf{2 0 0 4}$ & $\mathbf{2 0 0 5}$ & $\mathbf{2 0 0 6}$ & $\mathbf{2 0 0 7}$ & $\mathbf{2 0 0 8}$ & $\mathbf{2 0 0 9}$ \\
\hline 12.2 & 12.2 & 16.7 & 16.8 & 19.3 & 22.4 & 20.5 & 23 & 21 & 25.4 \\
\hline
\end{tabular}

The mean age of the patients tested $(40+/-2$ years $)$ and the proportion of male patients were unchanged during the study period.

CONCLUSION: HCV G1 is the dominant G in New Brunswick. The relative role of $\mathrm{G} 3$ increases over time. The population tested is otherwise stable in age and distribution of gender.

\section{P59}

COMPLETE GENOME SEQUENCING OF TWO COXSACKIE A9 VIRUS ISOLATES TO ASSESS RECOMBINATION EVENTS AND GENETIC DRIFT

K PABBARAJU ${ }^{1}$, S WONG ${ }^{1}$, R TELLIER ${ }^{1,2}$

${ }^{1}$ Provincial Laboratory for Public Health; ${ }^{2}$ Department of Microbiology, Immunology and Infectious Diseases, University of Calgary, Calgary, AB

OBJECTIVE: An unusually high incidence of enterovirus (EV) meningitis was seen in Alberta in 2010. Partial VP4 sequence revealed $81 \%$ of the isolates as coxsackie A9 (CVA9). Recombinations within the same species are common; to study this we determined and compared the complete genomic sequence of CVA9 isolates from 2003 and 2010.

METHODS: The complete genome of two CVA9 isolates were amplified by long RT-PCR. The amplicons were sequenced by genome walking.

RESULTS: Nucleotide (nt) comparison of the 2 isolates revealed the 5'UTR to be $96.5 \%$ identical; the VP (1,2,3 and 4) regions were $94.71 \%$ identical, the 3' UTR were $83.11 \%$ identical. There was more divergence in the domain encoding the $2 \mathrm{~A}, 2 \mathrm{~B}, 2 \mathrm{C}, 3 \mathrm{~A}, 3 \mathrm{~B}, 3 \mathrm{C}$ and $3 \mathrm{D}$ proteins. $\mathrm{Nt}$ identity in the $2 \mathrm{~A}$ and $2 \mathrm{~B}$ protein regions was $96.15 \%$ and $93.60 \%$ respectively, identity in the $2 \mathrm{C}, 3 \mathrm{~A}$ and $3 \mathrm{D}$ proteins was $\approx 80 \%$. Lowest nt identity was observed in the $3 \mathrm{~B}$ protein at $74.63 \%$ and $3 \mathrm{C}$ protease at $77.50 \%$. BLASTX comparison of the VPg protein to the protein database revealed $100 \%$ and $95 \%$ similarity of the 2003 and 2010 isolates to the VPg of other CVB and echoviruses. Similarly, the 3C protein of the 2003 isolate was $98 \%$ identical to that of CVB and some echoviruses, the 2010 isolate was $100 \%$ identical to that of EVs 97 and 75.

CONCLUSIONS: The CVA9 isolates showed a high level of homology in the 5'UTR, VP, 2A and 2B regions. Significant difference was noted in the non-structural region of the genome suggesting recombination events between the CVA9, other CVBs and echoviruses.

\section{P60}

EVALUATION OF THE FOCUS DIAGNOSTIC HERPESELECT 1 AND 2 ELISA IGG

A MAK $^{1}$, M PETRIC $^{1,2}$, M KRAJDEN $^{1,2}$, A SEVERINI ${ }^{3}$

${ }^{1}$ PHSA Laboratory, BCCDC; ${ }^{2}$ University of British Columbia, Vancouver, BC; ${ }^{3}$ National Microbiology Laboratory, Winnipeg, MB OBJECTIVE: The Focus HSV1/2 type specific serology assay was implemented to determine HSV-1 and HSV-2 exposure status in select patient specimens. Results for anti-HSV-2 can generally differentiate samples as reactive vs. non-reactive (NR); results for anti-HSV-1 were more ambiguous when compared to Western Blot (WB).

METHODS: The Focus HSV EIA was performed on sera reactive for anti-HSV-1 and/or -2 by the Dade-Behring assay. Sera that tested equivocal or NR by the Focus assay were submitted to the NML for HSV WB testing.

RESULTS: A total of 1166/1556 samples were reactive for HSV-1 and 726/1556 were reactive for HSV-2 using Focus kit. 106 samples were reactive for HSV antibody by the Behring assay, but equivocal (EQ) or NR by the Focus HSV $1 / 2$ kit for either HSV-1 and/or HSV-2 underwent WB testing at the NML. Using the manufacturer's EQ zone of signal to calibrator ratio of $0.9-1.10$, of 8 samples EQ for HSV-1 by the Focus assay, 3 were WB reactive. Modification of the Focus EQ range between 0.3-1.10 for HSV-1 yielded HSV-1 WB reactive serology in 31/51 samples. In contrast, of the 6 specimens that were HSV-2 EQ using the manufacturer's EQ zone, only 2 were reactive by WB. Using the modified 0.3 to 1.1 range for equivocal status, 4/29 EQ specimens were HSV-2 WB reactive.

CONCLUSIONS: Focus anti-HSV-1/2 assay results with ratios of $>1.10$ and those with ratios of $<0.3$ were shown to display concordant WB serostatus. Samples with ratios between 0.3 to 0.9 likely require WB testing to confirm serostatus especially with regard to anti-HSV-1.

\section{P61}

\section{A REVIEW OF THE CURRENT NLHRS HTLV SEROLOGY CONFIRMATORY ALGORITHM IN IDENTIFYING HTLV-II ANTIBODY POSITIVE SAMPLES}

\section{MALLOCH, J KIM}

PHAC, Ottawa, ON

OBJECTIVE: The NLHRS evaluated its current strategy to diagnose the HTLV-II antibody positive status of clinical samples received for reference testing from Canadian laboratories.

METHODS: The NLHRS uses two confirmatory tests (Innogenetics Inno-LIA HTLV I/II Score assay and in-house HTLV-1 RIPA) run in tandem to help resolve HTLV antibody status. The Inno-LIA is a synthetic peptide based discriminatory assay capable of differentiating between HTLV-I and HTLV-II while the HTLV-I RIPA is derived from whole viral lysate. Under the current regime, both of the confirmatory assays must concur or the sample is deemed indeterminate. Seven years of retrospective data from clinical samples submitted to the NLHRS for HTLV testing between January 2003 and August 2010 was examined for samples testing HTLV-II positive on the Innogenetics Inno-LIA HTLV I/II Score assay.

RESULTS: The NLHRS tested 64 HTLV-II antibody positive samples by both Inno-LIA and HTLV-I RIPA. Although 10 (15.6\%) samples were positive on HTLV-I RIPA, a discouraging 39\% (25 samples) tested negative on HTLV-I RIPA. The remaining 30 samples were indeterminate by RIPA.

CONCLUSION: It is known that HTLV-II cross-reacts with HTLV-I antigens, however our data demonstrates that the majority of HTLV-II samples do not cross-react with HTLV-I antigens. HTLV-II is a concern as the major association to HTLV-II positivity is intravenous drug use. The spread of blood borne pathogens is well documented in this high risk population. Although HTLV-II is not conclusively linked to disease, it is important to monitor this human retrovirus as it may evolve to a more pathogenic form. Laboratorians should be diligent in ensuring the HTLV testing algorithm they use has the ability to detect antibodies to HTLV-II as well as HTLV-I.

\section{P62}

METAGENOMIC INSIGHTS: PUBLIC HEALTH RISK POTENTIAL OF SIX UNTREATED WATER SAMPLES N PRYSTAJECKY ${ }^{1,2}$, J GARDY $^{2,3}$, M GRAHAM $^{4,5}$, K JEWELL $^{1}$, V WOLF $^{4}$, T MURPHY ${ }^{4}$, S TYLER ${ }^{4}$, J ISAAC-RENTON ${ }^{1,2}$, P TANG $^{1,2}$ ${ }^{1}$ British Columbia Centre for Disease Control Public Health and Reference Laboratory (BCPHL); ${ }^{2}$ University of British Columbia; ${ }^{3}$ British Columbia Centre for Disease Control (BCCDC), Vancouver, BC; ${ }^{4}$ National Microbiology Laboratory; ${ }^{5}$ University of Manitoba, Winnipeg, MB

INTRODUCTION: It is estimated that $99 \%$ of organisms present in the environment cannot be detected by traditional cultivation methods. Metagenomic analyses of nucleic acids derived from aquatic environments (groundwater, freshwater, marine water) allow exploration of ecological relationships and identification of novel microorganisms. For this work, we compare the bacterial metagenomic profiles of distinct aquatic communities, identifying sequences of clinical importance.

METHODS: Water samples were collected as part of routine water testing at British Columbia Public Health and Reference Laboratory (BCPHL). Six water samples were analyzed; four recreational water samples (two from marine beaches and freshwater beaches) and two surface water samples used for drinking water. Following isolation of genomic DNA, metagenomic profiles were created by $16 \mathrm{~s}$ rRNA targeted amplification, followed by sequencing on Roche's 454 sequencing platform at the National Microbiology Laboratory (NML). 
RESULTS: Over 800,000 reads were generated. Using NCBI BLAST, sequence identities to the species/isolate level were achieved for $40 \%$ of sequences. The majority of sequences represented common aquatic, nonpathogenic microorganisms, with marine and freshwater (beach and surface water) showing distinct metagenomic profiles. Microorganisms associated with enteric and soil environments were not detected in marine environments but were detected in beach and surface water samples. Some sequences of clinical importance that were detected include Campylobacter jejuni, Bacillus cereus, Burkholderia cepacia, Legionella pneumophila, Vibrio vulnificus and Mycobacterium avium complex.

DISCUSSION: This work represents early stages of novel environmental metagenomic work in Canadian water supplies. Bacterial pathogen sequences represent a small proportion of total sequences, particularly in comparison to common environmental organisms such as Rhizobium spp. However, their presence in these water samples emphasizes the role of water as an important transmission vehicle in infectious disease investigations.

\section{P63}

RURAL WELL WATER CONTAMINATION AND HUMAN HEALTH: AN INVESTIGATION IN EASTERN ONTARIO D RYDING ${ }^{1}$, G EVANS ${ }^{1,2}$, K MOORE ${ }^{1,3}$, A VANDIJK ${ }^{3}$, A MAJURY ${ }^{1,4}$

${ }^{1}$ Queen's University; ${ }^{2}$ University Hospitals Kingston; ${ }^{3}$ KFL\&A Health Unit, Kingston; ${ }^{4}$ Ontario Agency for Health Protection and Promotion, Toronto, Ontario

OBJECTIVE: The human health implications of bacterial contamination of drinking water across both a population and large aquifers are poorly understood. And, the role of bacterial, and contaminant migration, through complex aquifers is critical with respect to the study of microbial pathogens, and the mechanisms and risks related to their human consumption through contaminated water.

METHODS: Kingston PHL tests drinking water samples from private wells that are collected from the Hastings \& Prince Edward, Leeds, Grenville \& Lanark, and Kingston, Frontenac, Lennox \& Addington counties. The population in this area is approximately 480,000 residents. Waters are processed via membrane filtration and examined for the presence of E. coli or coliforms. Contaminated waters are further assessed, using rt-PCR, for the presence of $B$. fragilis, and typed by host species-human, bovine or other.

RESULTS: On average, Kingston PHL tests between 20,000 and 35,000 drinking water samples each year. Further, of the samples tested, between $30 \%$ and $40 \%$ have some form of bacterial contamination and of these, between $4 \%$ and $9 \%$ are contaminated with E. coli. Of the 378 specimens for which data are currently available, fecal contamination was tracked to human source in $61 \%$, bovine source in $0 \%$, both human and bovine in $4 \%$ and neither bovine or human in $35 \%$.

CONCLUSIONS: This study uses molecular techniques to track microbial sources with the intent of better understanding of both the source and causes of fecal contamination with the expectation of enabling more effective public health interventions.

\section{P64}

COMPARATIVE EVALUATION OF PURITAN AND COPAN LIQUID AMIES TRANSPORT SYSTEMS FOR THE RECOVERY OF STREPTOCOCCUS PNEUMONIAE AGAINST THE CLSI M40A STANDARD

\section{B GANDHI ${ }^{1}$, T MAZZULLI ${ }^{1,2}$}

${ }^{1}$ Department of Microbiology, Mt Sinai Hospital and University Health Network; ${ }^{2}$ University of Toronto, Toronto, ON

OBJECTIVE: To assess the performance of two modified liquid Amies transport systems, Puritan Liquid Amies Transport System with a standard flocked swab (P) (Puritan Medical Products Company LLC) and Copan's ESwab (E) (Copan Diagnostics Inc.) intended for processing using automated plating systems for the recovery of Streptococcus pneumoniae (SPN). METHOD: The CLSI M40A Roll-Plate method at room temperature was followed to test the viability of $13 \mathrm{SPN}$ isolates from the Toronto Invasive Bacterial Disease Network (TIBDN) stock as well as 2 ATCC strains of (SPN) ATCC 6303 and ATCC 49619 strains, for up to $48 \mathrm{hrs}$. The average colony counts for the 3 swabs for each organism/dilution/time combination were read manually at 0, 24 and $48 \mathrm{hrs}$ and compared against control plates. For viability studies to be considered acceptable there had to be $\geq 5$ CFU following the specified holding time from the specific dilution that yielded zero-time plate counts closest to $300 \mathrm{CFU}$.

RESULTS: At 10-3 dilution P recovered all 15 isolates after $48 \mathrm{hrs}$; E recovered one mucoid ATCC strain after $48 \mathrm{hrs}$ and five strains were recovered after $24 \mathrm{hrs}$. At $10^{-4}$ dilution P recovered 5 strains after $48 \mathrm{hrs}$ and 10 after $24 \mathrm{hrs}$. E recovered zero strains after $48 \mathrm{hrs}$ and five strains after $24 \mathrm{hrs}$.

CONCLUSION: This study shows: (1) recovery of SPN was reduced at lower dilution and (2) the P system appears to outperform the E system and meets the CLSI M40A requirements.

\section{P65}

INCIDENCE OF INVASIVE ASPERGILLOSIS AND

ANTIFUNGAL USE FOLLOWING IMPLEMENTATION OF GALACTOMANNAN IN A HIGH RISK HEMATOLOGY POPULATION USING THE 2008 EORTC CRITERIA C BEAUCHAMP-PAYMENT ${ }^{1}$, M DJADI ${ }^{1}$, DJG THIRION ${ }^{1,2}$, DC SHEPPARD ${ }^{3}$, Y ROUSSEAU $^{1}$, S CARLE $^{1}$, C DUPONT ${ }^{1}$, G POPRADI ${ }^{1}$, A BONNICI ${ }^{1}$

${ }^{1}$ McGill University Health Centre; ${ }^{2}$ University of Montreal;

${ }^{3}$ McGill University, Montreal, QC

BACKGROUND: Invasive aspergillosis (IA) is a major cause of mortality in high risk hematology patients. The recent development of the galactomannan (GM) assay, combined with standard investigation, can significantly improve the diagnosis of IA as presented in the 2008 EORTC updated criteria. This study evaluated IA incidence with implementation of GM testing. It also determined the impact of GM testing on antifungal use.

METHODS: A retrospective pre-post study of neutropenic patients with acute myelogenous leukemia (AML) or undergoing allogeneic hematopoietic stem cell transplant (HSCT) in the hemato-oncology unit of two tertiary care centers. GM testing was implemented by protocol in 2009. Outcomes: IA was diagnosed by blinded physician using the 2008 EORTC criteria for the baseline (2006-2008) and GM testing (2009) groups. Antifungal use was described by the mean duration of treatment in each group and by the rate.

RESULTS: For the AML population, 37 patients were included in the baseline group and 13 episodes in the GM group were included. In the baseline group, there were 4 patients with a probable diagnosis of IA (incidence of $10.8 \%$ ) and 7 possible cases of IA. In the GM group, there was 1 patient with proven IA, 1 probable (incidence of 15.4\%) and 3 possible cases. The mean duration of treatment was 13.4 days in the baseline group and 20.0 days in the post-GM group. The rate was calculated to be 0.189 in the baseline group versus 0.352 in the post-GM group $(t=-2.34, p=0.03)$. For the HSCT population, 28 patients were included in the baseline group and 9 patients in the GM group. In the baseline group, there were 2 patients who received a possible diagnosis of IA (Incidence not available). In the GM group, there was 1 patient with proven IA, 2 probable (incidence of $23 \%$ ) and 2 possible cases. The mean duration of treatment was 29.1 days in the baseline group and 23.8 days in the post-GM group. The rate was calculated to be 0.305 in the baseline group versus 0.485 in the post-GM group $(t=-0.11, \mathrm{p}=0.91)$.

CONCLUSIONS: Incidence of IA is clinically relevant in the AML and HSCT population. Antifungal use remained elevated after implementing of GM testing.

\section{P66}

MARAVIROC MEDIATED CCR5 BLOCKAGE FOR HIV INFECTED INDIVIDUALS IS ASSOCIATED WITH INCREASED RATES OF RESPIRATORY TRACT INFECTIONS Y KEYNAN ${ }^{1,2,3}$, E RUBENSTEIN ${ }^{1,2}$, K KASPER $^{1,2}$, K FOWKE ${ }^{1,3}$ ${ }^{1}$ University of Manitoba, Department of Medical Microbiology; ${ }^{2}$ University of Manitoba, Department of Internal Medicine; ${ }^{3}$ University of Manitoba, Department of Community Health Sciences, Winnipeg, MB

INTRODUCTION: Maraviroc is an allosteric, non-competitive blocker of CCR5, interfering with its ability to bind to the HIV GP120 and thus preventing HIV cell entry. CCR5 is involved in recruiting immune cells to sites of viral invasion in the respiratory tract. 
METHODS: This postulated role of CCR5 led us to perform a metaanalysis of the largest clinical drug trials that evaluated CCR 5 antagonists, as part of antiretroviral regimens, with focus on the occurrence of respiratory infections in general and viral infections in particular in CCR5 antagonist recipients. Clinical trials involving administration of Maraviroc to HIV infected individuals were reviewed with emphasis on reported respiratory infections. We included only trials that enrolled more than 100 individuals in the CCR 5 antagonist treatment arm.

RESULTS: We found in the clinical data significantly more respiratory infections in the CCR 5 blocker treated arm than in the control arm. The incidence was 11 LRTI; 7 PCP in the treatment arm compared to 1 LRTI; 0 PCP in controls. (Lower respiratory tract infections in the maraviroc treatment arms - OR 7.497, CI 0.9982-56.31), $\mathrm{p}=0.04$.

CONCLUSIONS: This analysis along with the increased recognition of the role CCR5 plays in trafficking of lymphocyte to the site of infection, suggests that the CCR5 blockade may lead to more infections caused by other viruses and respiratory pathogens in HIV infected patients and that the incidence of such infections needs to be carefully assessed.

STUDENT POSTER PRESENTATIONS

THURSDAY, APRIL 7 AND FRIDAY, APRIL 8 ROOM: MCGILL

\section{SP1}

ENDEMIC FOCUS OF NON-TOXIGENIC CORYNEBACTERIUM DIPHTHERIAE CAUSING CUTANEOUS DISEASE IN AN IMPOVERISHED URBAN COMMUNITY

\section{CF LOWE ${ }^{1}, \mathrm{~K}^{\text {BERNARD }}{ }^{2,3}, \mathrm{MG} \mathrm{ROMNEY}^{4,5}$}

${ }^{1}$ University of Toronto, Toronto, ON; ${ }^{2}$ National Microbiology

Laboratory, $\mathrm{PHAC}^{3}$ University of Manitoba, Winnipeg, MB;

${ }^{4}$ University of British Columbia; ${ }^{5}$ Providence Health Care,

Vancouver, BC

OBJECTIVES: To conduct a clinical, microbiological and molecular characterization of cutaneous diphtheria presenting to a large tertiary care hospital serving an impoverished urban community.

METHODS: A systematic chart review of patients presenting with cutaneous diphtheria between 1998 - 2007 was completed. C. diphtheriae isolated from wound cultures underwent confirmatory testing and diphtheria toxin studies at the provincial and national reference laboratories. Multilocus sequence testing (MLST) was performed on available isolates and compared to an online database of $\mathrm{C}$. diphtheriae sequences.

RESULTS: 33 patients were reviewed, of which 30 were residents of the impoverished community. Patients had a history of injection drug use (66.7\%) and multiple medical co-morbidities including HBV (18.2\%), HCV (63.4\%), HIV (33.3\%), recurrent ulcers (24.2\%) and psychiatric illnesses $(21.1 \%)$. One patient developed invasive disease (bacteremia and endocarditis). Wound cultures were primarily polymicrobial, with Staphylococcus aureus and Streptococcus pyogenes most commonly cocultured. All C. diphtheriae isolates were non-toxigenic. 29 isolates were available for MLST analysis. The predominant sequence type (ST) was $76(20 / 29$ or $69 \%)$. The remaining isolates were distributed between ST5 $(n=1)$, ST32 $(n=6)$, ST78 $(n=1)$ and ST81 $(n=1)$.

CONCLUSIONS: Cutaneous diphtheria was associated with injection drug use and low socioeconomic circumstances. Over a 10 year period, cutaneous disease due to non-toxigenic $\mathrm{C}$. diphtheriae has established itself as an endemic pathogen in this impoverished community.

\section{SP2}

PREDICTABILITY OF NOROVIRUS OUTBREAKS ACTIVITY

\section{IN ALBERTA}

M HASING ${ }^{1}$, B LEE ${ }^{1}, X$ PANG $^{1,2}$

${ }^{1}$ University of Alberta; ${ }^{2}$ Provincial Laboratory for Public Health,

Edmonton, $A B$

OBJECTIVES: We have published a unique biannual pattern of Norovirus (NoV) outbreaks in Alberta over an eight-year period (Jul 2000 - Jun 2008).
This pattern was characterized by alternating quiescent and epidemic years associated with the emergence of novel GII.4 variants in the epidemic years. In this study, we summarize the NoV outbreak data in Alberta for the winter months of 2008, 2009 and 2010 to test the predictability of the previously observed biannual pattern.

METHODS: Provincial Public Health Laboratory provides NoV testing using real-time RT-PCR for all stool samples submitted from suspected NoV outbreaks in Alberta and the Northern Territories. At least one positive specimen from each $\mathrm{NoV}$ positive outbreak was sequenced within the capsid gene. Nucleotide sequences were analyzed with the NoV Genotyping Tool Version 1.0 or NCBI DNA nucleotide blast for genotype identification. As NoV peaks in the winter and the winter season for 2010/11 is still incomplete, a 4-month period, Sep to Dec, from 2008 to 2010, was used to compare the NoV outbreaks activity.

RESULTS: The number and proportion of NoV positive outbreaks between Sep and Dec for each year was 89/122 (73\%) in 2008, 15/23 (65\%) in 2009 and 68/100 (68\%) in 2010 following the biannual pattern. NoV genogroup II (GII) was predominant in all three years, $90 \%, 80 \%$ and $99 \%$ of all $\mathrm{NoV}$ outbreaks, respectively. 2008 was an epidemic year and the major variant strain in circulation was GII.4 2006b, which was found in $85 \%$ of all NoV GII outbreaks. 2009 was a quiescent year and GII.4 2006b was found in only $25 \%$ of the NoV GII outbreaks, with non-GII.4 genotype being more common. The new GII.4 variant (GII.4 2010) was first identified in Nov 2009 but only had sporadic activity up to Mar 2010.

CONCLUSION: The biannual pattern of NoV outbreaks in Alberta has persisted since 2000 . NoV sequencing and typing can provide important information that can be used to predict NoV outbreak activity and enhance appropriate health resources allocation by public health officials. We predict GII.4 2010 will be the predominant strain in the winter of 2010/11.

\section{SP3}

\section{A LONGITUDINAL STUDY TO EXAMINE THE EPIDEMIOLOGY OF METHICILLIN-RESISTANT STAPHYLOCOCCUS AUREUS (MRSA) CONTAMINATION IN THE GENERAL ENVIRONMENT OF THREE COMMUNITY HOSPITALS IN ONTARIO}

M FAIRES ${ }^{1}$, D PEARL ${ }^{1}$, J WEESE ${ }^{1}$, W CICCOTELLI ${ }^{2,3}$, R SCHERTZBERG ${ }^{2}$, K STRAUS ${ }^{2}$, G ZINKEN ${ }^{3}$

${ }^{1}$ University of Guelph, Guelph; ${ }^{2}$ Grand River Hospital; ${ }^{3}$ St Mary's General Hospital, Kitchener, ON

OBJECTIVES: To estimate the prevalence of MRSA and identify surfaces that are more likely to be contaminated by MRSA in the general environment in three community hospitals in Ontario.

METHODS: In 2010, surfaces in the medical and surgical wards of three hospitals were sampled once a week for four consecutive weeks. Sterile electrostatic cloths were wiped over each environmental surface and processed using enrichment methods for MRSA. MRSA isolates were spa typed and examined for the PVL toxin gene using PCR. Patient MRSA isolates were also obtained. Risk factors analyzed included type of surface sampled and location using a multi-level logistic regression model.

RESULTS: Overall, 11.6\% (72/619) of surfaces were positive for MRSA. Relative to computer keyboards, surfaces significantly associated with MRSA contamination included chairs, hand rails, isolation carts, and sofas. Four different MRSA strains were identified among these isolates: CMRSA-2 (50\%), CMRSA-5 (34.7\%), spa type 539 (12.5\%), and CMRSA-10 (2.8\%). However, CMRSA-5 and spa type 539 were not found in any patient.

CONCLUSIONS: MRSA contamination was relatively common, but several environmental strains were not found in patients. The discordance between patient and hospital MRSA strains suggests unidentified reservoirs or sources, such as staff, visitors, or unscreened patients. Several surfaces had an increased risk of being contaminated with MRSA but further studies regarding contact rates among hospital surfaces, type of surface material, and the populations using these surfaces are warranted. 


\section{SP4}

SCCMEC TYPE IV MRSA ACQUISITION IN HOSPITAL SETTINGS: PORTRAIT FROM A UNIVERSITY HEALTH CENTER IN MONTREAL, CANADA

P MORENCY-POTVIN, D ROULEAU, M ROGER, S GAGNON, P St-ANTOINE, P LAPLANTE

Centre Hospitalier de I'Université de Montréal (CHUM), Université de Montréal, Montreal, QC

OBJECTIVE: Community Acquired Methicillin-resistant Staphylococcus aureus (CA-MRSA) has emerged in the late 1990s and is increasingly present in hospital settings. This study aims to determinate the importance of CA-MRSA genotypes in-hospital acquisition among patients in our institution.

METHODS: This was a retrospective chart review study from April 2009 to March 2010. MRSA isolates from the first clinical specimen of patients were collected. Demographics, clinical data and sensitivity profile were then retrieved from patient files. Staphylococcal cassette chromosome mec (SCCmec) typing and Panton-Valentine Leukocidin (PVL) detection were performed by PCR for healthcare-associated MRSA (HA-MRSA) defined by CDC's criteria.

RESULTS: Among all MRSA specimens, 312 were identified as HA-MRSA. 48 (15.4\%) SCCmec type IV strains (normally associated with CA-MRSA) and $2(0.6 \%)$ type I, $256(82.1 \%)$ type II and $1(0.3 \%)$ type III (classic strains of HA-MRSA) were found. 12 cases of nosocomial (defined as a positive culture more than $48 \mathrm{~h}$ after admission) CA-MRSA strains were reported. However, SCCmec type 4 strains were less likely to be nosocomial (OR 0.46). 85.4\% of SCCmec type IV carried the genes encoding PVL compared to only $1.1 \%$ in other types. $97.9 \%$ of type IV strains were sensitive to clindamycin compared to $3.4 \%$ in strains from types I, II and III.

CONCLUSION: A significant proportion of MRSA infections acquired in our institution are now caused by CA-MRSA genotypes. This has potential important clinical and infection control impacts.

\section{SP5}

NOSOCOMIAL TRANSMISSION OF GROUP B STREPTOCOCCI IN A NEONATAL INTENSIVE CARE UNIT PROVEN BY POSITIVE ENVIRONMENTAL CULTURE A AL-MAANI ${ }^{1}$, L STREITENBERGER ${ }^{2}$, M CLARKE $^{2}$, Y YAU $^{3}$, D KOVACH $^{3}$, R WRAY ${ }^{2}$, A MATLOW 1,2

${ }^{1}$ Division of Infectious Diseases, The Hospital of Sick Children; ${ }^{2}$ Infection Prevention and Control Department; ${ }^{3}$ Division of Microbiology, The Hospital for Sick Children, Toronto, ON OBJECTIVE: Neonates are particularly susceptible to Group B streptococcal (GBS) infection which is usually acquired vertically. Nosocomial transmission of GBS has also been reported to occur in neonatal care units. In January 2010, there were an increased number of clinical specimens from our neonatal intensive care unit (NICU) growing GBS. This study describes outbreak management and investigate role of environment contamination in nosocomial transmission of GBS.

METHODS: Microbiology laboratory records were reviewed to identify isolates of GBS from the NICU during December 2009 and January 2010. The patients from whom these specimens were collected were identified and their charts reviewed. Environmental samples from the unit were collected, and clinical and environmental isolates were compared by pulse field gel electrophoresis (PFGE). A point prevalence study was conducted twice before calling off the outbreak.

RESULTS: PFGE patterns of 3 clinical strains from 6 patients were indistinguishable. One environmental strain was isolated from a patient monitor, and had identical PFGE pattern to that of the three clinical strains. Infection control measures were implemented and follow-up unit point prevalence screening showed no new cases.

CONCLUSION: Although poor infection control practice has been implicated in previous reports of nosocomial outbreaks of GBS infection in NICUs, our finding provides unique evidence that the environment can act as a reservoir of GBS and play a key role in nosocomial transmission.

\section{SP6}

CLINICAL AND MICROBIOLOGIC CHARACTERISTICS OF STENOTROPHOMONAS MALTOPHILIA NOSOCOMIAL BACTEREMIA OVER AN ELEVEN YEAR PERIOD P NAIDU, S SMITH

University of Alberta Hospital, Alberta Health Services, Edmonton, AB

OBJECTIVES: To describe the microbiologic and clinical characteristics of S. maltophilia blood stream infections (BSI) over an eleven year period at a tertiary care centre in Canada.

METHODS: All adult S. maltophilia BSIs from 1999 to 2009 were identified through the Infection Control nosocomial infection surveillance program. Demographic and clinical data were extracted from the Infection Control database and from patient charts. Microbiologic data were confirmed through the laboratory information system.

RESULTS: Twenty five episodes of S. maltophilia BSI (0.9\% of all BSI), involving 24 patients, were identified between 1999 and 2009. The patient age range was 18 to 83 years (average 45.7 years). The majority were males $(14 / 24 ; 58.3 \%)$. The mean length of hospital stay was 83.3 days (range: $8-$ 310 days). The rate of S. maltophilia BSI s per 1000 admissions ranged from 0.04 to 0.22 with an average rate of 0.09 . More than half of the patients were admitted to the ICU $(13 / 25,52 \%)$ prior to BSI onset. Laboratory data were available on 24 of the 25 isolates. Polymicrobial infections were present in 11/24 (45.8\%) episodes. Resistance to trimethoprim/sulphamethoxazole (TMP/SMX) occurred in $8.3 \%$ of all infections. Fifteen percent of isolates were resistant to ticarcillin/clavulanate. Mortality attributed to S. maltophilia BSI was $16.7 \%$.

CONCLUSIONS: In our centre the rate of S. maltophilia BSI remains very low and constant over more than a decade. Patients generally had prolonged hospital stays and multiple comorbidities. The MICs for the TMP/SMX susceptible S. maltophilia strains remain stable and resistance rates are low.

\section{SP7}

THE POSITIVE CLINICAL IMPACT ON STAPHYLOCOCCAL BACTEREMIA BY DIRECT MECA PCR TESTING OF BLOOD CULTURE BOTTLES

B WANG ${ }^{1,2}$, P JESSAMINE $^{1,2}$, M DESJARDINS $^{1,2}$, B TOYE ${ }^{1,2}$, K RAMOTAR $^{1,2}$

${ }^{1}$ The Ottawa Hospital, Ottawa, ON ${ }^{2}$ The University of Ottawa, Ottawa, ON

OBJECTIVE: Prospective evaluation of the clinical impact of direct mecA PCR testing of positive BacT/Alert blood culture bottles with Grampositive cocci in clusters (GPCC) on optimization of antibiotic therapy. METHODS: Direct mecA PCR was performed on positive blood cultures with GPCC using the Roche Light-Cycler MRSA Kit, and results phoned to the attending physician. Susceptibility testing of the staphylococcal isolates was performed using the Vitek 2 P580 cards (bioMérieux). Analysis of antibiotic usage in response to lab results was performed on patients from July - December 2010. For evaluation purposes of oxacillin susceptible (OXS) staphylococcal bacteremia optimal therapy (OPTx) was defined as cloxacillin or equivalent beta-lactams; whereas optimal therapy of oxacillin resistant (OXR) staphylococci was defined as vancomycin, or linezolid. The use of vancomycin, or linezolid in the absence of a true allergy, for OXS staphylococci was considered as non-optimal therapy (NOPTx).

RESULTS: A total of 48 significant bacteremias were reviewed. 4 patients died/withdrew therapy prior to the availability of the final susceptibility result. MRSA was recovered in 4 of 34 S. aureus bacteremias, and 11 of 14 CoNS were OXR. The clinical impact results are as follows:

\begin{tabular}{lcccccc}
\hline & \multicolumn{2}{c}{$\begin{array}{c}\text { Empiric therapy } \\
\text { (48) }\end{array}$} & $\begin{array}{c}\text { Therapy after } \\
\text { direct } \text { mecA (48) }\end{array}$ & $\begin{array}{c}\text { Therapy after final } \\
\text { susceptibility } \\
\text { report (44) }\end{array}$ \\
\cline { 2 - 7 } & OPTx & NOPTx & OPTx & NOPTx & OPTx & NOPTx \\
\hline S. aureus (34) & 10 & 24 & 26 & 8 & 31 & 0 \\
CoNS (14) & 4 & 10 & 12 & 2 & 13 & 0 \\
Total & 14 & 34 & 38 & 10 & 44 & 0 \\
\hline
\end{tabular}


CONCLUSION: The implementation of direct mecA PCR testing improved the earlier optimization of antibiotic therapy in patients (38/48). Further improvements in optimizing therapy will require additional strategies, e.g. physician education or pharmacist intervention.

\section{SP8 \\ THE IMPACT OF NASAL CARRIAGE OF STAPHYLOCOCCUS AUREUS (SA) ON BLOODSTREAM INFECTIONS (BSI) IN A LARGE COHORT OF HEMODIALYSIS (HD) PATIENTS IN A TERTIARY-CARE CENTER}

JM LEDUC ${ }^{1,2}$, AS LAPOINTE ${ }^{1,2}$, M LEBLANC $^{1,2}$, AC LABBÉ 1,2 , K WEISS ${ }^{1,2}$

${ }^{1}$ Hôpital Maisonneuve-Rosemont; ${ }^{2}$ Faculté de médecine, Université de Montréal, Montréal, QC

OBJECTIVE: SA nasal carriage is a well-known risk factor for BSI in HD. We evaluated the impact of SA carriage regarding BSI and mortality while patients were on a screening and decolonization protocol. In vitro susceptibility of our SA strains to 10 antibiotics was also evaluated.

METHODS: HD patients were screened for SA carriage from September to December 2008 ( $n=360)$. Positive patients were treated with mupirocin for 5 days each month for 6 months. Charts were reviewed retrospectively for baseline clinical data (age, sex, time on HD, vascular access type, diabetes). Overall mortality and BSI were evaluated one year after the initial screening. Susceptibility testing to antibiotics including mupirocin and vancomycin was performed by agar dilution method.

RESULTS: Baseline characteristics were similar in carriers vs. non-carriers. BSI occurred in 14 patients (3.9\%). For SA, low (MIC>4 mg/L) and highlevel (MIC>512 mg/L) resistance to mupirocin was found in $3(4.5 \%)$ and $5(7.5 \%)$ strains, respectively. For vancomycin, all strains had a MIC $\leq 2 \mathrm{mg} / \mathrm{L}$

Table 1

\begin{tabular}{lcccl}
\hline & Total $(\mathbf{n}=\mathbf{3 6 0})$ & BSI : total & S. aureus BSI & $\begin{array}{c}\text { One-year } \\
\text { mortality }\end{array}$ \\
\hline SA carriers & $71(19.7 \%)$ & $3(4.2 \%)$ & $2(2.8 \%)$ & $11(15.5 \%)$ \\
Noncarriers & $289(80.3 \%)$ & $11(3.8 \%)$ NS & $6(2.1 \%)$ NS & $34(11.8 \%)$ NS \\
\hline
\end{tabular}

CONCLUSION: We found no significant differences in our cohort between SA nasal carriers and non-carriers in terms of BSI, SA BSI and overall mortality. Since SA carriers in HD usually have a higher risk of developing SA BSI, our study suggests that monthly application of mupirocin in carriers lowers the risk of BSI occurrence to the same level of noncarriers.

\section{SP9 \\ EVALUATION OF THE MICROBIOLOGY OF PERITONEAL DIALYSIS-ASSOCIATED PERITONITIS IN THE NORTHERN PERITONEAL DIALYSIS PROGRAM IN SASKATOON} A PABANI, S SKINNER, J BARTON

University of Saskatchewan, Saskatoon, SK

OBJECTIVES: The purpose of this study was to identify the epidemiological, historical, microbiological, and clinical factors that contribute to the development and outcome of peritonitis associated with peritoneal dialysis (PD) in the Northern Peritoneal Dialysis program (NPDP).

METHODS: A retrospective analysis of the NPDP from 2000-2010 was undertaken. Data including demographics, etiology of renal disease, type and duration of peritoneal dialysis, microbiology, treatment and outcomes were reviewed. Data were analyzed using SPSS software.

RESULTS: 539 episodes of peritonitis were reviewed. Coagulase-negative Staphylococci were the most common organism at $25.1 \%$. Staphylococcus aureus was the second at $19.7 \%$ with only $1.5 \%$ of total cases being MRSA. $11.3 \%$ were caused by gram negative bacilli. Risk factors for Staphylococcus aureus were Caucasian ethnicity ((Odds ratio 4.8 (95\% CI 1.3 - 19.0)), use of APD catheter ((OR $3.8(95 \%$ CI $1.3-11.2))$ and individuals with an exit site infection in the previous 3 months ((OR 4.4 (95\% CI 1.8 to 10.9)). CONCLUSIONS: Epidemiology of peritonitis in NDPD is unique with high rates of Staphylococcus aureus and low rates of MRSA and gram negatives. Staphylococcus aureus risk factors such as recent exit site infection are consistent with other studies; however, the finding of increased risk with Caucasian ethnicity and APD catheter use is unique and requires further research. Targeted review and enhanced treatment of exit site infections could reduce incidence of peritonitis in our program.

\section{SP10}

EVALUATION OF THE VITEK 2 SYSTEM FOR RAPID DIRECT IDENTIFICATION AND SUSCEPTIBILITY TESTING OF GRAM-NEGATIVE BACILLI FROM POSITIVE BLOOD CULTURES

$\underline{\text { S ABOUANASER }}{ }^{1}$, K NURI $^{1}$, P DRUMMOND ${ }^{2}$, L MONKMAN ${ }^{2}$, SE DALE ${ }^{1,2}, C_{\text {MAIN }}{ }^{1,2}$

${ }^{1}$ McMaster University; ${ }^{2}$ Hamilton Regional Laboratory Medicine Program, Hamilton, ON

OBJECTIVE: Rapid identification and susceptibility testing for GNB (gram negative bacilli) has the potential to improve patient therapy, outcome and improve workflow in the laboratory. To assess this, we evaluated direct identification of GNB using VITEK 2 (Biomerieux).

METHODS: Blood cultures with GNB identified in the BacT/Alert culture system (Biomerieux) were inoculated to the VITEK 2 ID-GNB card and AST-GN24 card following differential centrifugation. Identifications and susceptibilities were compared to standard identification methods.

RESULTS: Sixty positive aerobic blood cultures were evaluated. Fifty isolates were reported to a confidence level of $\geq 90(76 \%)$ and 10 isolates were reported to a confidence level of $<90$. Correct identification to the species level was achieved in $96 \%$ (48/50) when the confidence level was $\geq 90$.

Two misidentified organisms were A. calcoaceticus and E coli. Using MALDI-TOF, we were able to confirm the identification of 18 isolates directly from blood cultures. The susceptibilities of 48 strains had complete MIC agreement for $98.6 \%$ of the drugs tested. Of the disagreements, 1 had a very major error when a Citrobacter which was resistant to piperacillintazobactam was interpreted as susceptible, 3 had major errors and 6 were minor errors. Hands on technologist time was less than one hour.

CONCLUSIONS: This preliminary analysis found that direct identification and susceptibility testing by VITEK 2 may be a useful method for reporting gram-negative bacilli from blood cultures. This method shortens TAT, and may improve patient care with minimal increased cost to the laboratory.

\section{SP11}

FAILURE OF THE MODIFIED HODGE TEST (MHT) TO DETECT NEW DELHI METALLO-BETA-LACTAMASE-1 (NDM-1) CARBAPENEMASES

IV KUS ${ }^{1,2}$, BM WILLEY ${ }^{1}$, R MELANO ${ }^{2,3}$, C LAROCQUE ${ }^{1}$, K PIKE $^{1}$, M LUM $^{1}$, S HO$^{1}$, B MITCHELL ${ }^{1}$, C POULOS $^{1}$, E CUDEK $^{1}$, H DEDIER $^{1}$, IA EDWARDS ${ }^{1}$, D LOW ${ }^{1,2,3}$, A MCGEER ${ }^{1,2}$, SM POUTANEN ${ }^{1,2}$ ${ }^{1}$ University Health Network/Mount Sinai Hospital, Department of Microbiology; ${ }^{2}$ University of Toronto; ${ }^{3}$ Ontario Agency for Health Protection and Promotion, Toronto, ON

OBJECTIVE: NDM-1, a new carbapenemase identified in Enterobacteriaceae (ENT), has been spreading quickly worldwide. Rapid identification of NDM-1-producing strains is essential for its control. CLSI recommends the MHT for carbapenemase detection. We investigated the utility of the MHT for identifying NDM-1-producing strains. METHODS: From Nov. 1 to Dec. 31, 2010 ENT with ertapenem MIC $>0.5 \mathrm{mg} / \mathrm{L}$ (VITEK 2) or disk diffusion zones $<23 \mathrm{~mm}$ from a large clinical laboratory were tested by PCR to determine the presence of carbapenemase genes, including $b a_{\mathrm{NDM}-1}$. All bla $a_{\mathrm{NDM}-1}$ positive isolates were tested by the MHT following CLSI standards and interpreted by three medical laboratory technologists blinded to PCR results. Antibiotic susceptibilities were determined by in-house broth microdilution following CLSI.

RESULTS: In total, 57/2922 (1.9\%) ENT met our criteria for PCR; 4/57

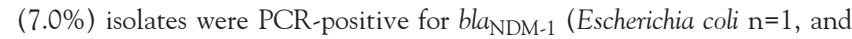
Klebsiella pneumoniae $\mathrm{n}=2$ (non-clonal), Morganella morganii $\mathrm{n}=1$ ). No other carbapenemases were detected. For 2 isolates, the MHT was interpreted as negative by the majority of readers, 2 were weakly positive. High 
levels of resistance to most classes of antibiotics were noted in all 4 isolates.

CONCLUSIONS: The MHT failed to detect 50\% of NDM-1-producing strains identified in a large clinical laboratory over a 2 month period. Clinical laboratories must be aware of the limitations of the MHT and seek alternate means to identify NDM-1-producing ENT so that timely identification can be made and infection control measures instituted accordingly.

\section{SP12 - WITHDRAWN}

\section{SP13 - WITHDRAWN}

\section{SP14}

EMBCAB POLYMORPHISMS IN RESISTANT MYCOBACTERIUM TUBERCULOSIS STRAINS THAT LACK EMBB306 MUTATIONS

\section{VOTH $^{1}$, J WOLFE ${ }^{2}$, S CHRISTIANSON $^{2}$, N SMART ${ }^{2}$,} M SHARMA ${ }^{1,2}$

${ }^{1}$ University of Manitoba; ${ }^{2}$ Public Health Agency of Canada, Winnipeg, MB

OBJECTIVE: Ethambutol (emb) resistance in Mycobacterium tuberculosis $(\mathrm{M} t b)$ has been associated with mutations in the embCAB operon. The most commonly observed mutations occur in the embB306 codon, but correlation with phenotypic resistance occurs in only $30-60 \%$ of the isolates. Also, embB306 mutations have been observed in phenotypically susceptible isolates.

METHODS: Twenty two Mtb isolates showing phenotypic resistance to emb and lacking a mutation within the embB306 codon were selected for this study. Sequencing of the entire $10 \mathrm{~kb}$ region containing the embCAB operon was accomplished using a series of primers selected from the literature.

RESULTS: Mutations within the embCAB operon were observed in 17 of 22 strains. Nine of those 17 had a mutation in the $e m b B$ gene, and 6 had multiple embB mutations. 13 of these mutations occurred between codons 355 to 497 of $\mathrm{embB}$, composing $59 \%$ of all isolates. Of the 2 strains lacking an $e m b B$ mutation, one had a mutation in codon 237 of embA and the other contained mutations in $e m b C$ codons 270 and 394. Further, 5 of the 7 emb resistant strains with wild type $e m b B$ sequences also displayed wild type sequences for the entire operon.

CONCLUSIONS: Mutations between codons 355 to 497 of embB were detected in 59\% of isolates lacking an embB306 mutation. Resistant strains lacking mutations within the $e m b C A B$ operon, suggest an alternate mechanism of emb resistance.

\section{ROOM: PALAIS}

\section{SP15}

\section{TREATMENT OUTCOMES IN PATIENTS WITH MRSA BACTEREMIA TREATED WITH VANCOMYCIN IN A TERTIARY CARE ACADEMIC CENTER} G GIRARD $^{1}$, EY HE ${ }^{1}$, DJG THIRION ${ }^{1}, 2$, VG LOO ${ }^{1}$, R HORN ${ }^{1}$, HG ROBSON ${ }^{1}$

${ }^{1}$ McGill University Health Centre; ${ }^{2}$ University of Montreal, Montreal, QC

INTRODUCTION: Mortality rates associated with invasive S. aureus infection ranges from 20 to $35 \%$. Vancomycin is currently the first line agent for treatment of known methicillin-resistant Staphylococcus aureus (MRSA) infection, and is now becoming the first line agent as empiric therapy of $S$. aureus because of increasing incidence of MRSA. The goal of this study was to assess predictive factors and clinical outcomes of patients with MRSA bacteremia in the Canadian context.

METHODS: This is a retrospective cohort study in MRSA bacteremic patients with clinical symptoms according to Centers for Disease Control defined criteria and treated with vancomycin from 2005 to 2010 in an academic hospital. Clinical and demographic information was collected for population description, analysis of risk factors for MRSA bacteremia, and treatment outcomes according to co-morbidities, control of the infectious source, MRSA sensitivities, and vancomycin therapy. MRSA strains extracted from blood samples were frozen for further testing. Outcomes evaluated included success to treatment and overall mortality. Vancomycin therapy was adjusted according to hospital protocol for targeted troughs of $10-15 \mathrm{mg} / \mathrm{L}$. Simple linear regression and Chi-square were used to identify factors associated with outcomes. Multiple logistic regressions were performed with all co-variates to identify influencing factors of outcomes.

RESULTS: 143 patients were identified to have MRSA bacteremia. 92 patients were included in the analysis. This was a medically heterogeneous population with an MRSA-Chronic Disease Score of 1.38. Successful outcomes were observed in $66.3 \%$ (61/92) patients treated with vancomycin. Overall mortality rate was $23.9 \%$ (22/92). Age was associated with mortality. Patients with Do-Not-Resuscitate orders had higher mortality and treatment failure rates. Mean duration of vancomycin therapy was 17.5 days. Vancomycin nephrotoxicity was observed in $18.5 \%$ (17/92) of patients. Outcomes were not associated with vancomycin levels; MRSA sensitivities to vancomycin were stable over time.

CONCLUSION: Patients with MRSA bacteremia had a lower mortality rate but slightly higher treatment failure rate compared to historical controls. Patient factors are strong predictors of outcomes.

\section{SP16}

CAN THE CLINICAL OUTCOMES ASSOCIATED WITH CLOSTRIDIUM DIFFICILE INFECTIONS (CDI) BE PREDICTED BY BACTERIAL GENOTYPE AND PHENOTYPES?

\section{S SIRARD, L VALIQUETTE, L-C FORTIER}

Université de Sherbrooke, Sherbrooke, QC

OBJECTIVE: Clostridium difficile is the main cause of nosocomial diarrhea. The NAP1/BI/027 hypervirulent strain emerged recently and was linked to several outbreaks. This strain is thought to produce more toxins, to better sporulate and to be associated with an increased risk of complications and relapses. The objective of this study was to determine if different outcomes associated with CDI can be predicted based on current typing methods and on virulence-associated phenotypes such as toxin production and sporulation.

METHODS: Twenty-one clinical isolates associated with variable CDI outcomes, including 14 ribotype 027 isolates (R027), were characterized by PCR-ribotyping, TRST, MLVA, PCR detection of toxin genes and tcdC gene sequencing. Toxin production was evaluated by immunodot blot and sporulation rates were determined in vitro by microscopic observations. RESULTS: The 14 R027 isolates were distributed amongst different CDI outcomes, including mild to moderate $(n=5)$, severe $(n=2)$, complicated $(n=4)$ and relapsing cases $(n=3)$. Ten isolates produced high quantities of TcdA and TcdB toxins in vitro, of which 9 had the typical 1-bp deletion in tcdC. The other R027 isolates produced less toxins despite a deleted tcdC. Moreover, toxin production and sporulation rates were generally inversely related, and the higher sporulating isolates were non-027 ribotypes.

CONCLUSION: Our results suggest that R027 isolates are not always associated with complicated or relapsing CDI cases. Also, virulence-associated phenotypes in vitro are not predictive of increased CDI severity.

\section{SP17}

\section{SERUM PROCALCITONIN AS A PREDICTOR OF} VENTILATOR-ASSOCIATED PNEUMONIA (VAP) $\underline{\text { S JOHN }}{ }^{1}$, B AHMED ${ }^{2}$, L KELLEHER ${ }^{2}$, C KIM $^{2}$, S ABOUANASER ${ }^{1}$, C LEE $^{1,2}$

${ }^{1}$ McMaster University; ${ }^{2}$ St Joseph's Healthcare, Hamilton, ON OBJECTIVE: The objectives of this study are as follows: to determine the accuracy of procalcitonin (PCT) levels to diagnose VAP, to assess the correlation between the Clinical Pulmonary Infection Score (CPIS) and PCT levels and to evaluate the relationship between PCT levels, antibiotic therapy and clinical improvement.

METHODS: From June 2009 to Dec 2010, patients expected to be intubated and ventilated for at least 7 days from an ICU in Hamilton were 
recruited to take part in a prospective, observational study comparing PCT levels and the CPIS. Endotracheal aspirates were collected on admission and every 72 hours of intubation until extubation or day 14 of intubation. Blood samples collected for routine patient care were used to measure PCT using an automated VIDAS instrument as per manufacturer's protocol. A chart review was performed to obtain patient demographics, antibiotic use and clinical data to determine the CPIS.

RESULTS: PCT $>10 \mathrm{ng} / \mathrm{ml}$ is correlated with a high likelihood of severe sepsis. CPIS $<6$ makes infection less likely. 45 patients were enrolled. Based on $2 \times 2$ contingency table, there were 60 observations where PCT was less than 10 AND CPIS less than or equal to 6; 11 observations where PCT was greater than or equal to 10 AND CPIS less than or equal to 6 . One observation where PCT was less than 10 AND CPIS greater than 6 . CONCLUSIONS: There is no defined gold standard to establish a diagnosis of VAP. Based on this preliminary data, we can conclude that when PCT is less than 10 one can rule out an underlying VAP.

\section{SP18 - WITHDRAWN}

\section{SP19}

\section{SERUM GALACTOMANNAN IN CYSTIC FIBROSIS PATIENTS} COLONIZED WITH ASPERGILLUS SPECIES

\section{T WARREN $^{1}$, Y YAU ${ }^{1,2}$, V WATERS ${ }^{1,2}$}

${ }^{1}$ University of Toronto; ${ }^{2}$ Hospital for Sick Children, Toronto, ON

OBJECTIVE: Colonization with Aspergillus spp. is a predictor for worsening lung function, hospitalization and infection post-lung transplant in cystic fibrosis patients. We sought to establish whether a galactomannan assay could detect circulating galactomannan in cystic fibrosis patients with chronic colonization and aid in identifying a subgroup that may need potential antifungal intervention.

METHODS: Serum samples from cystic fibrosis patients were collected prospectively over a two year period. We included sera from patients chronically colonized with Aspergillus spp. as well as those who had never had Aspergillus spp. cultured from a clinical specimen. All subjects were classified as: 1) Chronic Aspergillus; 2) Intermittent Aspergillus; 3) Never Aspergillus. The sera were tested for galactomannan using a one-stage immunoenzymatic sandwich microplate assay (Bio-Rad Laboratories Platelia ${ }^{\mathrm{TM}}$ Aspergillus EIA), following the manufacturers recommendations.

RESULTS: Seventy-five serum samples from 58 pediatric patients and 80 serum samples from 80 adult patients were tested. All serum samples had an optical density index $<0.5$ and were therefore considered negative for galactomannan. There was no difference in the mean optical density index between patients classified as chronic, intermittent or never colonized.

CONCLUSIONS: In our study, detection of serum galactomannan in cystic fibrosis patients was uniformly negative, in both adult and paediatric patients colonized with Aspergillus spp. and those not colonized.

\section{SP20 \\ IDENTIFICATION OF YEAST FROM BLOOD AND STERILE FLUID BY MATRIX-ASSISTED LASER DESORPTION/ IONIZATION TIME-OF-FLIGHT MASS SPECTROMETRY (MALDI)}

ISRIGLEY ${ }^{1}$, L MONKMAN ${ }^{2}$, S BURACOND ${ }^{2}$, L DALLE VEDOVE², C LEE $^{1,2}$, D YAMAMURA ${ }^{1,2}$

${ }^{1}$ Department of Pathology \& Molecular Medicine, McMaster University; ${ }^{2}$ Hamilton Regional Laboratory Medicine Program, Hamilton, ON

OBJECTIVES: MALDI is a rapid method for identification of organisms based on analysis of large organic molecules. This study assessed the accuracy of MALDI (Bruker Daltonics, Germany) in the identification of yeast from blood and sterile fluid cultures.

METHODS: Four protocols were tested on 18 well-characterized isolates using direct smears and extracted isolates from multiple media to optimize the identification scores. Consecutive blood and sterile fluid isolates from March-October 2010, and 8 additional isolates $(n=60)$, were then retrospectively identified using MALDI. Frozen isolates were subcultured twice to Sabouraud agar and incubated for 48 hrs. MALDI was performed on direct and extracted smears using Compass 1.2 SRI for FLEX series. Results were compared to conventional identification (microscopy, urea, Vitek2 YST, API20CAUX).

RESULTS: The clinical isolates tested were 12 different Candida species ( $\mathrm{n}=56)$, Trichosporon inkin and T. mucoides $(\mathrm{n}=2)$, and Cryptococcus neoformans $(\mathrm{n}=2)$. Based on results from the optimization phase, a score greater than 1.900 was found to be accurate for identification to the species level, which is lower than the manufacturer's recommendation of 2.300 to 3.000 . The majority of isolates (45/60) could not be identified by performing MALDI on a direct smear. Extracted isolates were correctly speciated in $96.7 \%$ (58/60). The 2 isolates (C. famata, C. saitoana) that were incorrectly identified were not in the MALDI database. The test procedure took 1-2 minutes on direct smears and 20-30 minutes on extracted isolates.

CONCLUSIONS: MALDI of extracted isolates is a rapid and accurate method for identification of clinically significant yeasts.

\section{SP21 - WITHDRAWN}

\section{SP22 - WITHDRAWN}

\section{SP23}

\section{COMPARISON OF THREE DIFFERENT METHODS FOR DETECTION OF SHIGA-TOXIN PRODUCING ESCHERICHIA COLI IN A TERTIARY PAEDIATRIC CARE CENTER} E VALLIERES, M ST-JEAN, F RALLU

Centre Hospitalier Universitaire Ste-Justine, Microbiology Department, Montreal, QC

OBJECTIVE: Shiga-toxin producing Escherichia coli (STEC) is a common cause of food-borne gastroenteritis, hemorrhagic colitis and may lead to haemolytic uremic syndrome (HUS). HUS is the major cause of acute renal failure in paediatric patients. STEC are characterized by the presence of at least one of the two types of Shiga toxins, Stx1 and Stx2. The first serotype of STEC identified was O157:H7 (O157 STEC) but at least 150 STEC serotypes have been associated with human illness. However, O157 STEC is the only serotype easily identified by culture on sorbitol MacConkey agar (SMAC). To optimize the recovery of non-O157 STEC, the CDC recommends since 2009 to screen for Shiga toxins all specimens submitted for enteric pathogens detection. The objective of this study was to compare the performance of the culture on SMAC with an enzyme immunoassay (EIA) and a real time PCR for the detection of STEC in our institution.

METHODS: Between June and September 2009, we tested all stool samples sent to our laboratory to rule out bacterial enterocolitis. The usual workup, including SMAC, for common enteropathogens was done, and a MacConkey broth was added. After overnight enrichment of the broth, the STEC screening was performed by EIA Premier EHEC (Meridian) and by a real time PCR targeting stx 1 and stx 2 . For EIA positive samples, the type of Shiga toxins was determined by the ImmunoCard STAT! EHEC (Meridian).

RESULTS: Of the 676 samples tested, 12 were positive for stx 1 by PCR. Only 3 of these specimens were positive by EIA ( 3 were Stx 1 by STAT!) and none was positive by SMAC. Two specimens were positive for stx 2 by PCR but were negative by EIA and SMAC. Finally, 7 specimens were positive for stx 1 and stx 2 by PCR. Among these 7 specimens, 5 were positive for O157-STEC by SMAC and only 3 were positive by EIA (by STAT!, 2 were negative and one was positive for Stx1 and Stx2).

CONCLUSION: Of 676 stool samples sent to our laboratory to rule out bacterial enterocolitis, we identified 21 STEC but only $24 \%$ was detected by culture on SMAC. This result goes along with the CDC guidelines which recommend that all stools submitted for testing from patients with acute community-acquired diarrhea should be assayed for non-O157 STEC with a test that detects the Shiga toxins or the genes encoding these toxins. 


\section{SP24}

PEPTIDOGLYCAN O-ACETYLTRANSFERASE AS A KEY VIRULENCE FACTOR OF STAPHYLOCOCCUS AUREUS IN A CAENORHABDITIS ELEGANS HOST MODEL

D TAMBALO ${ }^{1}$, K WU ${ }^{1}$, J-A MCCLURE ${ }^{2}$, J CONLY1,2, K ZHANG ${ }^{1,2}$

${ }^{1}$ University of Calgary; ${ }^{2}$ Alberta Health Services-Calgary, Calgary,

AB

OBJECTIVE: Peptidoglycan (PD) O-acetyltransferase (OatA) is considered a virulence factor in S. aureus. OatA confers resistance to lysozyme by modifying PD with addition of $\mathrm{O}$-acetyl groups. We sought to study the virulence of a wildtype (WT) and an oatA deletion mutant of methicillinresistant S. aureus (MRSA) in a C. elegans host model.

METHODS: A clinical isolate of the predominant CA-MRSA strain USA300 with demonstrated virulence in the C. elegans host model was used. An oatA deletion mutant was constructed by gene replacement. The virulence of WT, mutant, and complemented strains were analyzed in a C. elegans model. Data were analyzed by the Kaplan-Meier method and by the log rank test (GraphPad Prism). We also assessed the survival of the WT and oatA mutant strains after phagocytosis by human neutrophils and examined intracellular survival by live cell imaging using phase contrast microscopy.

RESULTS: The WT was resistant to lysozyme but the oatA mutant was sensitive. In the C. elegans host model, the WT and oat A mutant were around $90 \%$ and $29 \%$ nematocidal, respectively within 5 days. Compared with the WT, the oatA mutant demonstrated decreased survival post neutrophil phagocytosis, with $77 \%$ and $8 \%$ vs. $38 \%$ and $8 \%$ after 25 and 60 minutes, respectively.

CONCLUSION: This study showed that OatA is a significant virulence factor for S. aureus infections in a C. elegans model. It appears that resistance to lysozyme may be an important virulence mechanism in evading the innate immune system as in human or other mammalian host, further supporting the use of the C. elegans model for studying the virulence of S. aureus.

\section{SP25 \\ A PHASE II STUDY OF ${ }^{18} \mathrm{~F}$-FLUORODEOXYGLUCOSE IN ADULTS WITH PYOGENIC VERTEBRAL OSTEOMYELITIS USING PET/CT}

AK CHU $^{1,2}$, HR RABIN ${ }^{1,2}$, R KLOIBER $^{1,2}$, J HABA ${ }^{1,2}$

${ }^{1}$ University of Calgary; ${ }^{2}$ Alberta Health Services, Calgary, AB

OBJECTIVE: Current imaging modalities for the diagnosis of vertebral osteomyelitis include conventional xrays, bone scans, WBC scans, CT scans, and MRI with the latter being the imaging procedure of choice owing to its high sensitivity and specificity. However, resolution of MRI abnormalities often lags behind clinical improvement making it of limited use in assessing treatment response. There are several studies using ${ }^{18} \mathrm{~F}$ FDG PET/CT showing high sensitivity and specificity in diagnosing vertebral osteomyelitis. The objective of this study was to confirm the high sensitivity and specificity of ${ }^{18} \mathrm{~F}-\mathrm{FDG} \mathrm{PET} / \mathrm{CT}$ for the detection of pyogenic vertebral osteomyelitis, to determine if ${ }^{18} \mathrm{~F}$-FDG uptake more closely parallels the activity of the infection, and to determine the value of ${ }^{18} \mathrm{~F}$ FDG PET/CT in the treatment of patients who are not responding appropriately to treatment.

METHODS: Patients with clinical symptoms of vertebral osteomyelitis +/specific localization by an imaging study were enrolled and ${ }^{18} \mathrm{~F}-\mathrm{FDG} \mathrm{PET} /$ CT scans were performed. The extent of infection was quantified using the Standardized Value Uptake (SUV). Blood cultures and/or biopsy was performed and patients were treated for 4-6 weeks with pathogen-specific antibiotics, or otherwise empirically if no pathogen was identified. A repeat PET/ CT scan was performed at 8 weeks and the SUV was correlated with clinical response.

RESULTS: Of the 20 patients enrolled, 5 had PET/CT showing no infection and had no organism recovery. After no clinical progression over a 6 month followup, they were deemed to be true negatives. 11/15 patients with PET/CT showing vertebral osteomyelitis had a microbial pathogen identified. The other 4 were empirically treated and improved, making infection likely. Overall sensitivity was $100 \%$ and specificity was $100 \%$. Of the $9 / 15$ patients who returned for repeat PET/CT after receiving antibiotic therapy, 8 had clinically improved with an average SUV $\max$ decrease of 2.87. One patient with worsening clinical symptoms and increasing $\mathrm{SUV}_{\max }$ required laminectomy and prolonged antibiotics.

CONCLUSION: ${ }^{18} \mathrm{~F}-\mathrm{FDG} \mathrm{PET} / \mathrm{CT}$ is a highly sensitive and specific modality for the diagnosis of vertebral osteomyelitis. Furthermore, FDG uptake provides an objective way to assess activity of infection and response to treatment.

\section{SP26 - WITHDRAWN}

\section{SP27}

I GET BY WITH A LITTLE HELP FROM MY FRIENDS PEER TEACHING OF ANTIMICROBIAL TREATMENT OPTIONS IN MEDICAL SCHOOL

\section{BELOZER, S FORGIE}

University of Alberta, Edmonton, $\mathrm{AB}$

OBJECTIVES/BACKGROUND: The Year 1 medical curriculum at the University of Alberta contains a 7-week block of microbiology. There are a wide variety of teaching modalities including 21 hours of large-group bacteriology sessions. For half of these sessions, students presented antimicrobial treatment options for specific bacterial infections.

METHOD: The 8 minute presentation had to be engaging, describe the top antimicrobials for the bacterial infection, explain how the antimicrobials work, and outline resistance issues. They were marked with a specific scoring rubric, and a questionnaire was administered.

RESULTS: Group presentations encompassed a wide variety of techniques including skits, videos and songs. All groups chose antimicrobials wisely, and $89 \%$ named three other antimicrobial choices. $44 \%$ provided a precise explanation about how the antimicrobials work and $56 \%$ provided a credible description of how antimicrobials work and resistance issues. $89 \%$ communicated information in a persuasive manner using aids that engaged the audience. The average overall mark was $95 \%$. Of the 120 who responded to the survey, $83 \%$ stated the presentations were the most enjoyable parts of the course because they were a "creative way to summarize material covered in class", "good group bonding" "Interactive, humorous and above simply memorizing facts." All of the presentations were recorded and each group created a handout.

CONCLUSIONS: Students responded positively to peer teaching. The repetitive nature of the information coupled with fun presentations engaged the audience and promoted the wise use of antimicrobials.

\section{SP28 \\ NOT A JEDI YET, BUT NOT A PADAWAN EITHER: NEAR-PEER TEACHING OF MICROBIOLOGY W CHAN, S FORGIE}

University of Alberta, Edmonton, AB

BACKGROUND: Residents are taught to be clinical teachers, but receive little experience in lecturing to students. The Year 1 medical \& dental curriculum at the University of Alberta contains a 7-week course in microbiology and immunology, including laboratory sessions in microbiology. A new series of medical microbiology sessions was trialled with 29 dental students. These were taught by a resident, who by virtue of his 'near-peer' status was hypothesized to be able to bring a fresh take on clinical microbiology.

METHOD: 33 first-year dentistry students attended the lecture series. The first session was a didactic lecture, an overview of medical microbiology and considerations from a dental perspective. The second was a game show designed to review prior concepts and to introduce new ones. Both sessions were designed and delivered by the near-peer instructor, a medical microbiology resident, and reviewed by the course coordinator. After the second session, a questionnaire was administered.

RESULTS: Response rate was $88 \% .90 \%$ agreed that they enjoyed the sessions. 97\% agreed that they liked having a near-peer instructor, with 86\% preferring a near-peer instructor over a more senior teacher. 90\% believed they increased their understanding of microbiology, and 86\% thought that the material covered was relevant to their field of study.

CONCLUSIONS: Students responded very positively to this session 
series, and were especially positive regarding teaching from a near-peer. We show that residents can be effective near-peer teachers of medical and health care students in larger groups and lecturer roles.

\section{SP29 \\ ENVISIONING A NEW CURRICULUM FOR POST GRADUATE MICROBIOLOGY PROGRAMS}

INADARAJAH, A SIMOR, T MAZZULLI, L MATUKAS

University of Toronto, Toronto, $\mathrm{ON}$

OBJECTIVE: The first 6 months of postgraduate Microbiology training are important in providing a foundation in Microbiology and inspiring trainees' ongoing learning. The goal is to develop a microbiology curriculum that will meet these needs.

METHODS: To determine the key components of a curriculum that would address trainees' needs, a questionnaire was distributed to 39 trainees; current and graduates since 2005 in Adult and Paediatric Infectious Diseases, Medical (MM) and Clinical Microbiology (CM). Participants were asked to rate components of their microbiology training using a 5 point scale and to comment on their training.

RESULTS: A response rate of $72 \%(n=28)$ was achieved with representation from all the programs. Bench rotations were rated very useful by $61 \%$ $(\mathrm{n}=17)$, however, comments suggested improvements were needed in molecular techniques, parasitology and mycology. 71\% $(\mathrm{n}=20)$ rated workup of unknowns and plate rounds as very useful. $75 \%(n=21)$ found teaching sessions with the microbiologist very useful and suggested more time. Lab management meetings were rated as useful mainly by $\mathrm{CM}$ and MM trainees. Most respondents who performed call and sign-out duties found it somewhat useful. Infection control was rated a somewhat useful component by most participants and was suggested to be expanded. $57 \%$ $(n=16)$ rated a research project as very useful. Other recommendations included a more directive curriculum with standardization across teaching sites.

CONCLUSION: Highly rated components and common recommendations will be incorporated into a new curriculum to improve Microbiology training.

\section{SP30 \\ FIRST REPORT OF XENOPHILUS AEROLATUS CAUSING CLINICAL INFECTION IN A PATIENT WITH CAPD PERITONITIS}

N THAMPI $^{1}$, K BERNARD $^{2}$, B NG ${ }^{2}$, E HARVEY $^{1}$, Y YAU $^{1}$

${ }^{1}$ The Hospital for Sick Children, Toronto, ON; ${ }^{2}$ National

Microbiology Laboratory, Public Health Agency of Canada,

Winnipeg, MB

OBJECTIVE: Xenophilus aerolatus is a newly described member in the family Comamonadaceae. First identified from air samples in South Korea, it has never been associated with any clinical infections. We described here the first human infection secondary to $X$. aerolatus in a four-year-old boy with catheter-related peritonitis and the characterization of this organism.

METHODS: Peritoneal fluid was inoculated directly onto a combination of agar plates. The bacterial isolate (NML100527) was further characterized by conventional biochemicals, gas chromatography and 16S rRNA sequencing. Antimicrobial susceptibility testing was performed by broth microdilution. RESULTS: A patient with end-stage renal failure, on CAPD, presented with acute abdominal pain and fever one day after returning from a 10-day trip to Hawaii and Connecticut. 4 of 4 peritoneal fluid cultures were positive for $X$. aerolatus over a 20-day period. Pinpoint yellow colonies were isolated from Columbia sheep blood and chocolate agar incubated at $35^{\circ} \mathrm{C}$ in $\mathrm{CO}_{2}$ after 2 days. There was no growth anaerobically. The isolate was motile, Gram-negative and appeared coccobacillary to short rods in shape. It was oxidase and catalase positive, but nitrate, gelatin, cetramide, esculin, and triple decarboxylases negative. It was asaccharolytic and hydrolyzed xanthine, hypoxanthine, and tyrosine. 16S rRNA sequencing showed $100 \%$ homology with the type strain X. aerolatus 5526S-2T. Cellular fatty acids by GLC were consistent with published results. It appeared susceptible to aminoglycosides, quinolones, carbapenems, and trimethoprim/sulfamethoxazole, but resistant to aztreonam and pipercillin.
CONCLUSION: This is the first report of a clinical infection associated with X.aerolatus. The patient improved on a 4-week course of ciprofloxacin.

\section{SP31}

\section{FIRST REPORT OF ISOLATION AND CHARACTERIZATION OF PIGMENTIPHAGA DAEGUENSIS FROM A CLINICAL SAMPLE}

N BRIDGER $^{1,2}$, T BURDZ $^{3}$, D WIEBE ${ }^{3}$, K BERNARD $^{2,3}$

${ }^{1}$ Department of Pediatrics and Child Health; ${ }^{2}$ University of Manitoba; ${ }^{3}$ Special Bacteriology Section, National Microbiology Laboratory, Public Health Agency of Canada, Winnipeg, MB OBJECTIVES: A 6-year old girl from northern Manitoba with a history of liver transplantation presented to a local emergency department in 2008 with otalgia and otorrhea. She was diagnosed with suppurative otitis media. She had been swimming in an indoor pool within 48 hours of symptom onset. A swab of purulent material from her ear was sent to the microbiology laboratory for culture and sensitivity. The culture yielded 4+ Streptococcus pneumoniae, 3+ Staphylococcus aureus, and 1+ Gram negative bacilli (GNB) which could not be identified using conventional phenotypic systems. The isolate was sent to a reference laboratory where a polyphasic approach was undertaken for further characterization.

METHODS: Standard phenotypic, chemotaxonomic, and 16S rRNA gene sequencing were employed by the reference laboratory. Antimicrobial susceptibility testing was performed with broth microdilution using Sensititre GN2F and GPN3F panels and CLSI interpretative standards for GNBs.

RESULTS: The isolate (NML 080357) was strictly aerobic, motile, catalase positive, oxidase positive, and otherwise chemically inert. The strain was identified by nearly full $16 \mathrm{~S}$ rRNA gene sequencing and was found to have $99.5 \%$ identity to the $P$. daeguensis type strain. The cellular fatty acid composition analysis of the strain was consistent with the type strain of $P$. daeguensis. The isolate was multidrug resistant.

CONCLUSIONS: We believe this is the first clinical case involving P. daeguensis isolated from a clinical sample and potentially associated with human infection.

CLINICAL VIGNETTE POSTERS
THURSDAY APRIL 7 AND FRIDAY APRIL 8
ROOM: MCGILL

\section{CVP1}

FEVER, LEUKOCYTOSIS AND ZOONOTIC EXPOSURE DIAGNOSING LEPTOSPIROSIS

LSALMON $^{1}$, D WEBSTER ${ }^{1,2}$, S EL-BAILEY ${ }^{2}$

${ }^{1}$ Department of Medicine, Division of Infectious Diseases;

${ }^{2}$ Department of Medical Microbiology, Saint John Regional Hospital, Saint John, NB

PRESENTATION: A 47-year-old male with a past history of COPD and chronic stable back pain presented to hospital complaining of a 1-week history of fevers and chills, diffuse fine maculopapular rash, blurred vision and retro-orbital headaches. He lived in a rural area but has some exposure to sick contacts. He had a pet dog as well as a half-wild raccoon that came in and out through a pet door and whose litter box was under this patient's bed. He also had exposure to a sick owl 48 hours prior to onset of the illness. He lacked risk factors for blood-bourne infections and had lived in Canada his entire life.

INVESTIGATIONS: Upon admission, the patient was dehydrated with some mild acute renal insufficiency that resolved with fluid resuscitation. He had leukocytosis: $14.5 \times 10^{9} / \mathrm{L}$ that increased to $23.6 \times 10^{9} / \mathrm{L}$ on his $5^{\text {th }}$ day of admission and then gradually normalized. Chest radiographs showed no acute abnormality and a CT scan of the chest, abdomen and pelvis showed some mild lymphadenopathy in the mediastinum only. Two sets of blood cultures and urine cultures from admission were negative as were follow-up blood cultures. Q-fever serology, EBV serology, CMV serology, 
mycoplasma serology, cryptococcal serology and leptospira serology were sent.

TREATMENT: The patient was started on IV ceftriaxone and clindamycin and switched to cefuroxime axetil on the $5^{\text {th }}$ day of admission. He completed a 10-day course. On the $7^{\text {th }}$ day of admission, azithromycin was added and continued to complete a 5 -day course. He was discharged from hospital on the $8^{\text {th }}$ day after admission as his symptoms and his leukocytosis were resolving.

FOLLOW-UP: Serology for leptospira was positive (microagglutination test positive for serogroups Australis and Grippotyphosa). By one month post-admission, the patient had returned to his pre-morbid state of health. Leptospira serological testing on his bed partner was negative.

\section{CVP2}

GRANULOMAS...SO EVOCATIVE YET SO UNSPECIFIC! J FRÈRE $^{1}$, F LE DEIST ${ }^{2}$, A HATAMI ${ }^{3}$, E HADDAD ${ }^{2}$, D BOURON-DAL SOGLIO $^{4}$, V LAMARRE ${ }^{1}$, P OVETCHKINE ${ }^{1}$

${ }^{1}$ Infectious Diseases Division, Department of Pediatrics; ${ }^{2}$ Immunology Division, Department of Pediatrics; ${ }^{3}$ Dermatology Unit, Department of Pediatrics; ${ }^{4}$ Department of Histology and Pathology, CHU Sainte-Justine, Université de Montréal, Montréal, QC

PRESENTATION: A 5-year-old boy was transferred to our institution for a peri-anal abscess with neutropenia. Upon exam, nodular skin lesions located on the internal sides of both thighs and feet were noted. These lesions first appeared when he was 6 months old. The child was from rural Quebec, born to non consanguineous French Canadian parents. He did not live on a farm and had no pets. There was no TB contact. During infancy, he had recurrent upper respiratory tract infections. Multiple biopsies of the cutaneous lesions were performed and pathological studies concluded to a granulomatous process with tuberculoïd granuloma formation. However, neither specific stains (including Ziehl-Niehlsen and WarthinStarry), nor cultures (including bone marrow culture) identified any infectious agents responsible for these pathological findings. The initial workup revealed auto-immune neutropenia and thrombocytopenia, hepatitis and enlarged mediastinal lymph nodes. After ruling out the diagnosis of chronic granulomatous disease, in absence of proven infection, the hypothesis of sarcoidosis-like process was retained and therefore steroids were started. Despite the treatment, the disease progressed with over the years involvement of liver, spleen and lungs. All these organs that were biopsied led to the same pathological findings: granulomatous process without any infectious agent identified. Concomitantly, he developed a severe immunodeficiency with a global T-cell lymphopenia (lymphocytes $0.9 \times 10^{9} / \mathrm{L} \mathrm{CD} 4+0.09 \times 10^{6} / \mathrm{L}$ ) with normal B-cell count and an excess of NK-cells. The working diagnosis was then an immune deregulation with immunodeficiency and auto-immunity. As the disease progressed, he underwent an unrelated umbilical cord blood transplant. Three months post-transplantation, as immunological reconstitution began, he presented to hospital with a history of high fever $\left(39^{\circ} \mathrm{C}\right)$, chills and abundant watery diarrhea.

INVESTIGATIONS: His labs revealed: WBC $20.29 \times 10^{9} / \mathrm{L}$, neutrophils $12.3 \times 10^{9} / \mathrm{L}, \mathrm{HB} 89 \mathrm{~g} / \mathrm{L}$, platelets $479 \times 10^{9} / \mathrm{L}$. Gastroscopy and colonoscopy excluded a graft versus host disease. Total body gallium scan showed increased uptake of the radiotracer in the region of the left mesenteric lymph node which was confirmed by CT scan. Excisional lymph nodes biopsy was performed. The Ziehl-Niehlsen stained samples revealed the presence of acid fast bacilli. Cultures of the samples were positive for Mycobacterium avium. Moreover, blood cultures, cultures of the stools, the urine and the sputum grew Mycobacterium avium, more than 3 years after the onset of the disease, when the child's T-cell immunity was starting to recover.

TREATMENT: A multiple drug regimen including Amikacin, Ethambutol, Clarithromycin and Rifampicin was therefore started. Amikacin was promptly stopped because of the occurrence of hearing loss.

FOLLOW-UP: Antimicrobial therapy was discontinued after ten months. No treatment failure or relapse were observed. The child is now in perfect health, his skin lesions are healed, his liver function tests and CBC are normal.

\section{CVP3}

MYCOBACTERIUM CELATUM SKIN INFECTION WITH SPOROTRICHOID SPREAD IN A HIV-NEGATIVE IMMUNOCOMPROMISED MAN - ANOTHER FISHING STORY...

JM LEDUC, C LAVALLÉE, SF DUFRESNE, AC LABBÉ, L POIRIER

Département de microbiologie-infectiologie, Hôpital MaisonneuveRosemont, Montréal, QC

PRESENTATION: A 68-year-old male presented to our hospital for a two-month history of progressing, painful plaques on his right arm. The patient was known for a transfusion-dependant myelodysplastic syndrome, type-II diabetes mellitus and a recently diagnosed temporal arteritis treated with prednisone and azathioprine. He had accidentally punctured the tip of his fourth finger with a fish-hook while on a fishing trip in Quebec a few weeks prior to the appearance of the first lesion.

INVESTIGATIONS: On admission, physical examination was remarkable for edema, erythema and warmth around the distal interphalangeal joint of the right fourth finger. There were also three erythematous, indurated and tender plaques with a sporotrichoid spread on his right arm without lymphadenopathy nor any other noticeable skin lesion. His white blood-cell count was $1.6 \times 10^{9} / \mathrm{L}$ (neutrophils $1.3 \times 10^{9}$ ). C-reactive protein was elevated at $83 \mathrm{mg} / \mathrm{L}$. A skin biopsy showed lymphocytic dermatitis without involvement of the epidermis and without granuloma. An MRI of the upper right limb confirmed that the infection was limited to the skin and soft tissue. One week after admission, a fluctuation was felt underneath the fourth finger lesion and an aspiration was conducted. It showed few acid-fast bacilli (AFB).

TREATMENT: The patient was started empirically on a regimen covering nontuberculous mycobacterias and received clarithromycin, rifampin and ethambutol. The aspiration and biopsy eventually yielded Mycobacterium celatum. Rifampin was stopped as the pathogen was resistant.

FOLLOW-UP: Six months later, treatment with clarithromycin and ethambutol was discontinued, as only residual hyperpigmentation had been present for the past three months. However, on follow-up one month later, there was relapse of erythema and warmth over the right elbow. A new biopsy demonstrated granulomatous inflammation and still yielded AFB, that were again identified as M. celatum. Therapy with trimethoprimsulfametoxazole, amikacin and moxifloxacin was started, with some improvement. A few weeks later, the patient died of rhinocerebral mucormycosis. 
A

Abouanaser S. . . . . . . . . . P6,SP10,SP17

Adam $\mathrm{HJ} \ldots \ldots \ldots \ldots \ldots \ldots \ldots$ E1,L2

Ahmed B ........... J4,P16,P21,SP17

Ahmed R ........................ 5

Ahmed-Bentley J . . . . . . . . . . . . . . . P5

Alawi M....................... J4

Alexander D. . . . . . . . . . H1,P45,P46,P56

Alfa M ...................... 66

Al-Harthi K.................. P37

Allen $\mathrm{V} \ldots \ldots \ldots \ldots \ldots \ldots \ldots \ldots . \ldots \ldots$

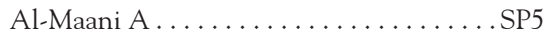

Almohri H. . . . . . . . . . . . . . . . . J1,J2

Al-Rawahi $G \ldots \ldots \ldots \ldots \ldots \ldots$ A6

Amihod B .................... I3

Andonov A .................... I1

Andonova M . ............... H5,H6

Antonishyn N . . . . . . . . . . . . . . . P8

Armstrong D . ................... P26

Arora $A \ldots \ldots \ldots \ldots \ldots \ldots \ldots$ G2

AstraKianakis G. . . . . . . . . G5,G6,P42

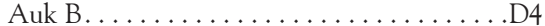

\section{B}

Babwah J .................. P48

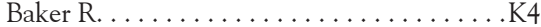

Balbirnie A. ................ H4,P30

Balfour L. . . . . . . . . . . . . . . . . . G4

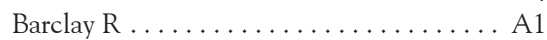

Bard JD ..................... P26

Barton J . . . . . . . . . . . . . . . . SP9

Bastien N ..................... P46

Beauchamp-Payment C............. P65

Bechard C . . . . . . . . . . . . . . . . J1

Beirnes J . . . . . . . . . . . . . . . . . P55

Bélanger-Trudelle É . . . . . . . . . . . . . P44

Béliveau C . . . . . . . . . . . . . . . . B6

Belozer C ........................SP27

Beniprashad M................. P57

Beresford N . . . . . . . . . . ........ 3

Bernard K. . . . . . . E4,H3,P8,SP1SP30,SP31

Biers K . . . . . . . . . . . . . . . B1

Bilawka J .....................C4

Bishop L....................... P19

Bittante J .................... J2

Blondel-Hill E ........... A2,A3,D2,P30

Bonnici A .................... P65

Borgundvaag B................ P22

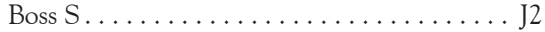

Bourgault A-M............... P31,P35

Bouron-Dal Soglio D . . . . . . . . . . . CVP2

Bowes $\mathrm{V} \ldots \ldots \ldots \ldots \ldots \ldots \ldots \ldots \ldots \ldots$

Bowie W.............A2,A3,A5

Boyd D . . . . . . . . . . . . . . D1,E3

Brandt K. . . . . . . . . . . . . . . P52

Bridger N . . . . . . . . . . . . . . . . . SP31

Brooks A . . . . . . . . . . . . E 5,P10

Broukhanski G. ...........P13,P14,P16

Brown K......................

Brown SZ . . . . . . . . . . . . K1

Bryce E. . . . . . A4,D1,E3,G5,G6,P34,P42

Bunzeluk K................... A1

Buracond S. . . . . . . . . . . . . SP20

Burdz T............E4,H3,P8,SP31

\section{$\mathrm{C}$}

Campbell J .

Canadian Nosocomial Infection

Surveillance Program ... A4,E2,G5,G6,P2,P34,P42

Cantin R .................... F6

Cardona M................... P57

Carle S........................ P65
Carranza D.................... J3

Carrière $S \ldots \ldots \ldots \ldots \ldots \ldots \ldots \ldots$ P17

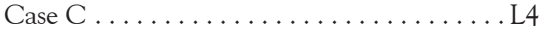

Castro M ...................... J3

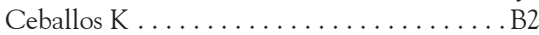

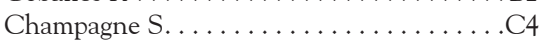

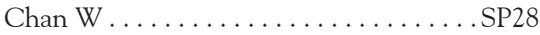

Chang Y . . . . . . . . . . . . . . . . P55

Charest H. . . . . . . . . . . B6,F6,P44,P51

Chernesky M . ............... B1,P53

Childerhose L ..................... B7

Chong M ...........A2,A3,A5

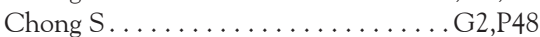

Christianson S . ............ P18,SP14

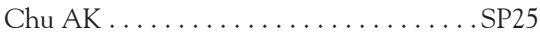

Chui $\mathrm{L} \ldots \ldots \ldots \ldots \ldots \ldots$ D5,F3,F4,L4,P11

Ciccotelli W....................SP3

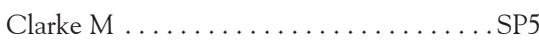

Coldman A . . .................... 2

Comité Sur Les Infections

Nosocomiales Du Québec .............. P33

Conly J . . . . . . . . . . . . . . . . . . . . E2,SP24

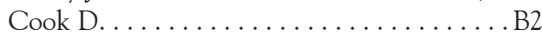

Corace K ................... G4

Couillard M................ B6,P44

Cross C. . . . . . . . . . . . . . . . H2

Cudek E.................... SP11

\section{$\mathrm{D}$}

Dale SE .................. P50,SP10

Dalle Vedove L . . . . . . . . . . . . . . . SP20

Daly J . . . . . . . . . . . . . . . . . . J3

Dandjinou W...................... P40

Dawson L.....................P12

Day $\mathrm{T} \ldots \ldots \ldots \ldots \ldots \ldots \ldots \ldots \ldots \ldots \ldots . . . \ldots \ldots$

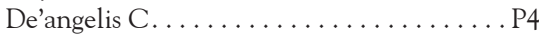

Decorby M . . . . . . . . . . . . . E E1,L2

Dedier H. . . . . . . . . . . . . . . . . . . . SP11

Deeks S. . . . . . . . . . . . . . . . . . . P56

Delorme M.................... P40

Delport J. . . . . . . . . . . . . . . . . . . P8

Demczuk W . . . . . . . . . . . . . . . . L2

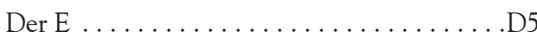

Desjardins M .............C1,C5,SP7

Deslandes S . . . . . . . . . . . . . . . . F5

Devlin HR . . . . . . . . . . . . . . P14

Dextras P . . . . . . . . . . . . . . F5

Dhaliwal S .................. A6

Dimitrova K................ H5,H6

Dines I . . . . . . . . . . . . . . . K2

Djadi M ...................... P65

Donaldson T . . . . . . . . . . . . . A1

Doucette K.....................P3

Douglas L.......................F4

Dowhaniuk D. . . . . . . . . . . . . . .C2

Drebot MA. . . . . . . . . . . . . H5, H6

Dreher K. . . . . . . . . . . . . A2,A3

Drews $S \ldots \ldots \ldots \ldots \ldots \ldots \ldots \ldots \ldots \ldots \ldots \ldots$ F4,K4

Drummond P . . . . . . . . . . . . . . . SP10

Dufresne SF . . . . . . . . . . . . . CVP3

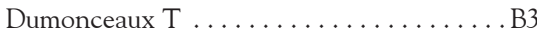

Dupont C........................ P65

Durand J...................... P41

\section{$\mathrm{E}$}

Ecobichon-Morris A . .............. P53

Edwards IA . . . . . . . . . . . . . . P22,SP11

El-Bailey S . . . . . . . . . . . . . . CVP1

Elit $\mathrm{L} \ldots \ldots \ldots \ldots \ldots \ldots \ldots \ldots \ldots \ldots \ldots \ldots \ldots$

Elligsen M ..................... P4

Elton T ..................... P1

Embil J . . . . . . . . . . . . . . E2,P2

Embree $J \ldots \ldots \ldots \ldots \ldots \ldots \ldots$ D1,E3,P2
Eshaghi A. . . . . . . . . . . . P45,P46,P56,P57

Eskandarian $\mathrm{S} \ldots \ldots \ldots \ldots \ldots \ldots \ldots \ldots \ldots \ldots$

Evans $G \ldots \ldots \ldots \ldots \ldots \ldots$ P47,P63

Fagan $\mathrm{A} \ldots \ldots \ldots \ldots \ldots \ldots \ldots \ldots \ldots \ldots$

\section{$\mathrm{F}$}

Faheem A. ...................... 3

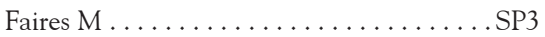

Fast M. .................... A1

Fauvel M .................. P44

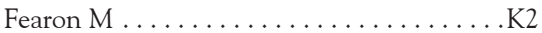

Fedirko L . . . . . . . . . . . . . . . P12

Fernandez E . . . . . . . . . . . . . . . B7

Fernando $\mathrm{K} \ldots \ldots \ldots \ldots \ldots \ldots \ldots \ldots \ldots \ldots \ldots \ldots$

Ferroni $G \ldots \ldots \ldots \ldots \ldots \ldots \ldots \ldots$ L 3

Fisman D . . . . . . . . . . . . . D6, L1

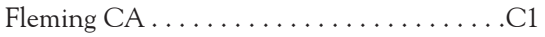

Flynn $\mathrm{G} \ldots \ldots \ldots \ldots \ldots \ldots \ldots \ldots \ldots \ldots$

Forgie $S \ldots \ldots \ldots \ldots \ldots$. P41,SP27,SP28

Fortier L-C . . . . . . . . . . . . . . . . . SP16

Fortin C . . . . . . . . . . . . . . P31

Fowke $\mathrm{K} \ldots \ldots \ldots \ldots \ldots \ldots \ldots \ldots$. . . . . . . . .

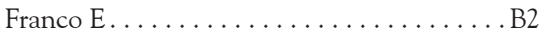

Frappier M ................... P17

Frenette $\mathrm{C} \ldots \ldots \ldots \ldots \ldots \ldots \ldots \ldots \ldots$. I3

Frère $J \ldots \ldots \ldots \ldots \ldots \ldots \ldots \ldots \ldots$ CVP2

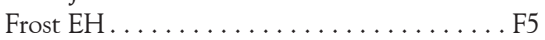

Fryters $S \ldots \ldots \ldots \ldots \ldots \ldots \ldots \ldots$. . . . . . . .

Fuller J . . . . . . . . P P, P23,P29,D4,F3

Fung $\mathrm{T} \ldots \ldots \ldots \ldots \ldots \ldots$ C3,P24,P25

G

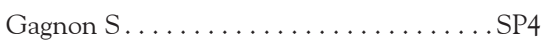

Galarneau L-A. . . . . . . . . . . . . . . . P35

Gandhi B ..................... P64

Garber G . . . . . . . . . . . . . . G4

Garceau R . . . . . . . . . . . . . . P58

Gardy J . . . . . . . . . . . . . . . P62

Gee S ......................... P5

Gelanis E .................... P32

Gelosia A . . . . . . . . . . . . D3,P29,P36

Gerbasi A.................... P32

Gervais R . . . . . . . . . . . . . G5,G6,P42

Ghosh C. . . . . . . . . . . . . . . . . . . .C2

Gilbride S................... P41

Gilchrist J. . . . . . . . . . . . . . . B1,P53

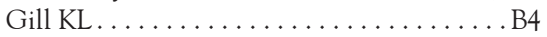

Gilmour M.............. H2,L2,P32

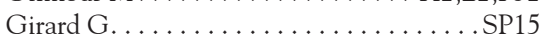

Girouard G. . . . . . . . . . . . . . . P58

Gnanasuntharam P . . . . D3,P22,P24,P25,P36

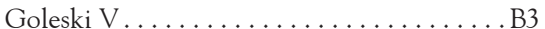

Graham M . . . . . . . . . . . . . H2,P62

Granados A . . ............... G2

Gravel D ... A4,D1,E2,E3,G5,I3,P2,P34,P42,G6

Gray $M \ldots \ldots \ldots \ldots \ldots \ldots \ldots \ldots \ldots \ldots$

Grice D. . . . . . . . . . . . . ....K1

Gubbay J. . . . . . . . . G3,P45,P46,P56,P57

Gunadasa K . . . . . . . . . . . . . . . . P55

Gun-Munro J . . . . . . . . . . . . . . . . C1

Guzman M..................C2

Haba J......................SP25

Haddad E . . . . . . . . . . . . . . . . . CVP2

Hadwell V . ........................ D2,P30

Haider $S \ldots \ldots \ldots \ldots \ldots \ldots \ldots$ E5,J4,P10

Haldane D . . . . . . . . . . . . . . . . . P28

Halwani M................... P37

Hamilton $\mathrm{K} \ldots \ldots \ldots \ldots \ldots \ldots \ldots \ldots$. B

Hamula C....................F1

Hansen G................... A1

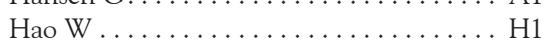




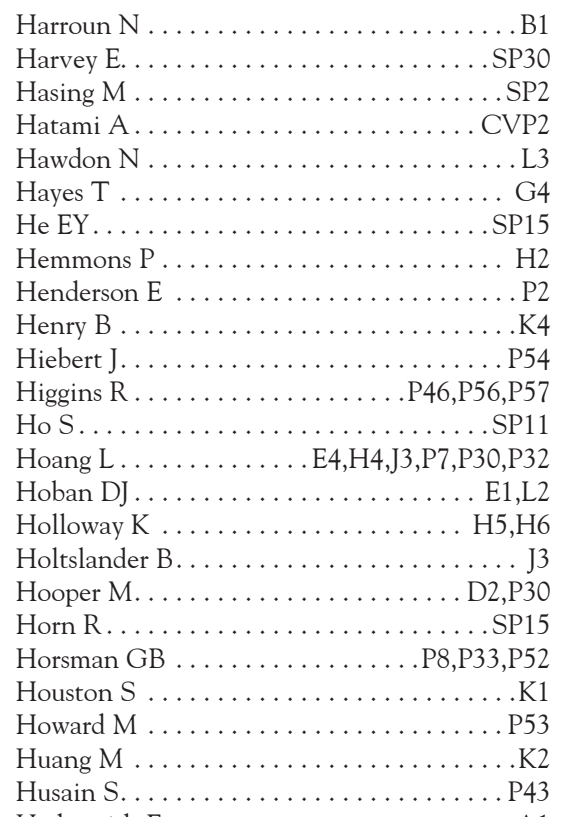

Hydesmith E. . . . . . . . . . . . . A1

\section{I}

Isaac-Renton J

$\mathrm{D} 4, \mathrm{~K} 3, \mathrm{P} 62$

\section{$\mathrm{J}$}

Jackson C...................... P14

Jalo I. . . . . . . . . . . . . . . . . . . . I4

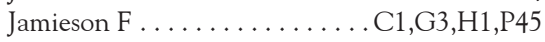

Jang D. . . . . . . . . . . . . B B, P53

Jang $\mathrm{W} \ldots \ldots \ldots \ldots \ldots \ldots \ldots \ldots \ldots \ldots \ldots \ldots$

Jayaratne $\mathrm{P} \ldots \ldots \ldots \ldots \ldots \ldots \ldots$ P15,P16

Jessamine $K \ldots \ldots \ldots \ldots \ldots \ldots \ldots$.C5

Jessamine P...................SP7

Jewell K ................... P62

John M.................... P2

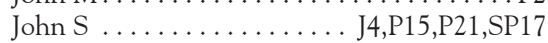

Johnson A . . . . . . . . . . . . . . P38

Johnson D . . . . . . . . . . . . . . P50

Johnson R . . . . . . . . . . . . . . J3

Johnston L . . . . . . . . . . E2, G5,G6,P2,P42

Johnstone J................. P6

$\mathrm{K}$

Kadivar K. . . . ...B

Kadkhoda K.................H5,H6,P20

Kanji J . . . . . . . . . . . . . . . I2,P5,P9

Kapala J . . . . . . . . . . . . . . B1,C2

Karlowsky JA . . . . ...............E1

Karnauchow T.................. P49

Kasper K. . . . . . . . . . . . . . . . . . . P66

Katz K................. C1,D3,P19

Kearney AK ................... P32

Kelleher L. . . . . . . . . . . . . P21,SP17

Kelly M. . . . . . . . . . . . . . . A6,J2,P40

Kern C ...................... P49

Keynan Y . . . . . . . . . . . . . . . . P66

Kibsey P . . . . . . . . . . . . . D1,E3,P7

Kim C. ................. J4,P21,SP17

Kim J .................... B5,P61

Klingel M..................... P53

Kloiber R ......................... SP25

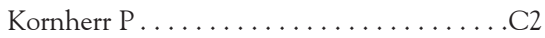

Koshman S.................P1

Kou A........................ P47

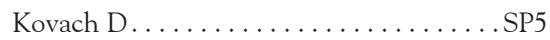

Kowalewska-Grochowska K. . . . . . . . . K1

Krajden M . . . ..........B2,G1,P55,P60

Kramer J................... P12
Kreiswirth N. . . . . . . . . . . . . P36

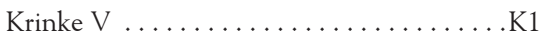

Kristjanson E . . . . . . . . . . . . . P45,P56

Kuhn M ................. D1,E3

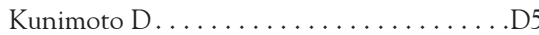

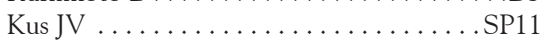

Kwong P..................... P11

\section{$\mathrm{L}$}

Labbé AC. ............... SP8,CVP3

Lagace-Wiens P . . . . . . . . . . . . E1,C6

Laing NM. . . . . . . . . . . . . . . . L2

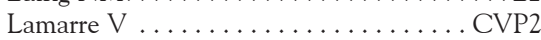

Lamirande R. . . . . . . . . . . . . . . B6

Lamothe F . . . . . . . . . . . . . . . P31

Laplante P ..................... . . . .

Lapointe AS. . . . . . . . . . . . . . . SP8

Larocque C. . . . . . . . . . . . . . C3,SP11

Laryea $\mathrm{K} \ldots \ldots \ldots \ldots \ldots \ldots \ldots \ldots$. A 1

Lathia N........................ P4

Lavallée C . . ....................................

Lawoyin $\mathrm{T} \ldots \ldots \ldots \ldots \ldots \ldots \ldots$. . . . . . . .

Le C ..................... F1

Le Deist F. . . . . . . . . . . . . . CVP2

Le Saux N . . . . . . . . . . . . . . . P2

Leblanc M .......................SP8

Leduc JM . . . . . . . . . . . . . SP8,CVP3

Leduc $\mathrm{S} \ldots \ldots \ldots \ldots \ldots \ldots \ldots$ E2,G5,G6

Lee $\mathrm{B} \ldots \ldots \ldots \ldots \ldots \ldots \ldots \ldots$. . . . . . . . .

Lee C . . . . . . J4,P15,P16,P21,SP17,SP20

Lee M-C. . . . . . . . . . F3,P5,H4,K3,K4

Lefebvre B . . . . . . . . . . . . P31,P35

Lepiane F . . . . . . . . . . . . . . . P4

Leung $\mathrm{W} \ldots \ldots \ldots \ldots \ldots \ldots \ldots \ldots . \ldots \ldots$ P22

Lévesque $\mathrm{S} \ldots \ldots \ldots \ldots \ldots \ldots \ldots$. . . . . . . . . . . .

Levett PN. . . . . . . . . . . H5,P8,P33,P52

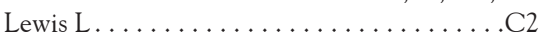

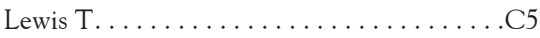

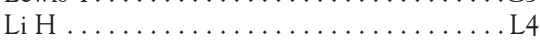

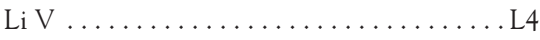

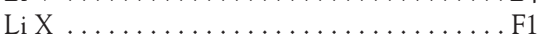

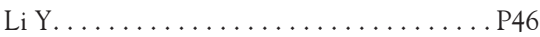

Lim G.................... P56

$\operatorname{Lin} \mathrm{L} \ldots \ldots \ldots \ldots \ldots \ldots \ldots \ldots \ldots \ldots \ldots \ldots \ldots \ldots \ldots \ldots$
Lo $\mathrm{P}, \mathrm{P} 22, \mathrm{P} 24, \mathrm{P} 25$

Lo $\mathrm{T} \ldots \ldots \ldots \ldots \ldots \ldots \ldots \ldots \ldots \ldots \ldots \ldots \ldots, \mathrm{K} 3, \mathrm{~K} 4$

Loeb M................ A4,B7,P34

Loftus M................. P22

Lombardi N ................... P45

Lombos E . . . . . . . . . . . . . . . G3,P45,P56

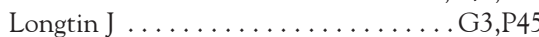

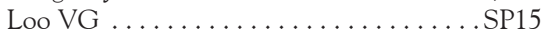

Lorenzana I. . . . . . . . . . . . . . . . . . . B7

Lortie $\mathrm{M} \ldots \ldots \ldots \ldots \ldots \ldots \ldots \ldots \ldots$ P44

Louie L... . . . . . . . D3,F2,G2,P24,P25,P34

Lovagi $\mathrm{G} \ldots \ldots \ldots \ldots \ldots \ldots \ldots \ldots$. . . . . . .

Low D. . . . . . . G3,H1,P36,P45,P46,P56,SP11

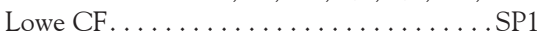

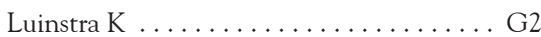

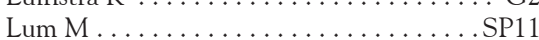

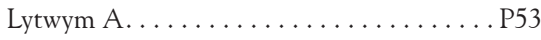

\section{$\mathrm{M}$}

Ma J ...................... H1

Macoretta B................... P21

Macpherson M................ P50

Mahli G.................... P22

Mahony J . . . . . . . . . . . . . . G2,P48

Main C. . . . . . . . . . . . . . . . SP10

Majury A . . . . . . . . . . . . . P47,P63

Mak A ............G1,P55,P60,P13

Makowski K................. H5,H6

Malejczyk K.....................F3

Mallet M ..................... P58
Malloch L. . . . . . . . . . . . . . . . . P61

Malott $\mathrm{S} \ldots \ldots \ldots \ldots \ldots \ldots \ldots \ldots \ldots . \ldots \ldots$ J3

Man S. .................... $44, \mathrm{~K} 4$

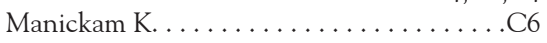

Mar W ...................... P20

Marchand-Austin A . . . . . . . . . G3,P45

Maregmen J . . . . . . . . . . . . . . P56

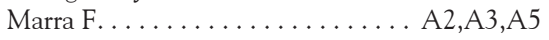

Martin I . . . . . . . . . . . . . . . L2,P31

Martinez L . . . . . . . . . . . . . . . A6

Masney S . . . . . . . . . . . . . . . P57

Mason A ................... I1

Mataseje LF . . . . . . . . . . . D1,E1,E3

Mathur S ..................... P48

Matisz C....................... 1

Matlow A............. A4,P2,P34,SP5

Matukas L. . . . . . . . . . . . . . . . . . . SP29

Mayo P............................ 1

Mazzocco A .....................J3

Mazzulli T. . . . . . . . . . . . . P25,P56,P64,SP29

Mccarthy A ................ G4

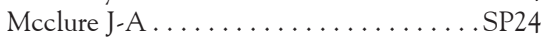

Mccready W....................L3

Mcdonald RR. ................... P33

McGeer A . . . ...................SP11

Mcgeer AJ . . . . A4,C3,D3,I3,P22,P24,P25,P34

Mckay R..............A2,A3,A5

Meaney H. . . . . . . . . . . . . . . . . . . .C1

Mei $W \ldots \ldots \ldots \ldots \ldots \ldots \ldots \ldots$ B2

Melano R......................SP11

Ménard J. . . . . . . . . . . . . . . . P51

Miedzinski L. . . . . . . . . . . . . . . . . . . . P9

Miller M.................. P26,P31

Millette M ....................P17

Mina N................. E4,P7

Minnema B ................... P36

Minnings K ................... P47

Mitchell B . . . . . . . . . . . . . . . . . SP11

Mitchell R ..........E2,G5,G6,I3,P42

Mithani S. . . . . . . . . . . . . . . H4

Monkman L . . . . . . . . . P15,P16,SP10,SP20

Moore D. . . . . . . . . . . . . . . . . . . I3

Moore K................... P63

Moreau D........................ D5

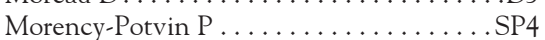

Morshed MG ..............H4,K3,K4

Mounchili A ........... A4,D1,E3,P34

Mubareka $S \ldots \ldots \ldots \ldots \ldots \ldots \ldots$ G2

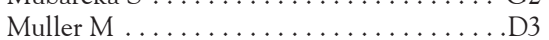

Mulvey M. ...... A4,D1,E1,E2,E3,P33,P34

Murphy D.................. I1

Murphy T................. P62

\section{$\mathrm{N}$}

Nadarajah J . . . . . . . . . . . . . . . . . SP29

Nagle E. . . . . . . . . . . . . . . P8,P33

Naidu P .................. K1,SP6

Ndao M ...................... 2

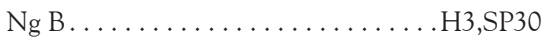

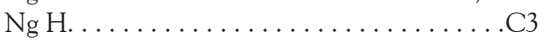

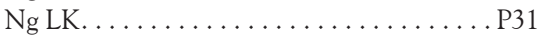

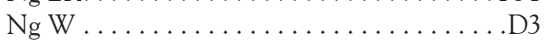

Nguyen G. ................... P22

Nichol KA .....................L2

Nigrin J. . . . . . . . . . . . . . P5

Nix E ......................L3

Noble M. ................. P27

Nuri K ......................SP10

$\mathrm{O}$

Ogbulafor K................. P56

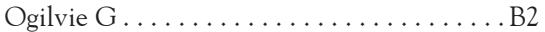

Onishi R................... P21

Onuma K.................... P53 
Ormiston D ......................E2

Ostrowska K.....................D3

Ovetchkine P................ CVP2

\section{$\mathbf{P}$}

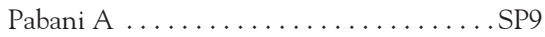

Pabbaraju K . . . . . . . . . . . . . . . B4,P59

Paccagnella A .................. J3

Pacheco AL . . . . . . . . . . . . .... H3

Pagotto F . . . . . . . . . . . . . H2

Palmay L. ......................E6

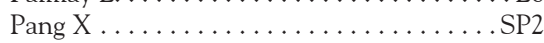

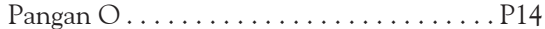

Patel S . . . . . . . . . . . . . G3,P45,P46,P56

Patrick D ............A2,A3,A5

Pearl D . . . . . . . . . . . . . . . . . SP3

Peralta A . . . . . . . . . . . . . . . P13

Petric M ................ G1,P55,P60

Petrich A ..................... G2

Pienaar C . . . . . . . . . . . . . . . . A 6

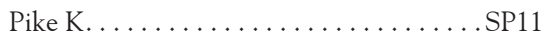

Pillai DR ...............P13,P14,P16

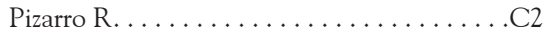

Poirier $\mathrm{L} \ldots \ldots \ldots \ldots \ldots \ldots \ldots \ldots \ldots \ldots \ldots \ldots \ldots$

Poliquin M.................... I1

Pong-Porter S. . . . . . . . . . . . . . . C3

Popradi G. . . . . . . . . . . . . . . . P65

Porter C . . . . . . .................. P29

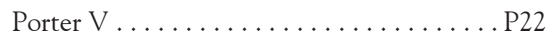

Poulos C......................SP11

Poutanen SM . . . . . . . . C1,C3,P22,P24,

P................P25,P29,P36,SP11

Prayitno K . . . . . . . . . . . C3,P24,P29

Prematunga $\mathrm{C} \ldots \ldots \ldots \ldots \ldots \ldots$ G4

Prystajecky N . . . . . . . . . . . . . . . . D4,P62

Purych D ................... P7

\section{$\mathrm{R}$}

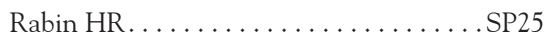

Racey S. . . . . . . . . . . . . . . P56

Rahim T......................

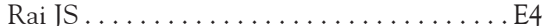

Rallu F ...................... SP23

Ramotar K . . . . . . . . . . . . . . . . SP7

Rasin NA. . . . . . . . . . . . . . . . . . P37

Rawte $\mathrm{P} \ldots \ldots \ldots \ldots \ldots \ldots \ldots \ldots \ldots$. . . . . . . . . . . . . .

Raymondo D . . . . . . . . . . . . . . . . . . C1

Reimer A ................ H2,H3

Rennie R . . . . . . . . . . . . . . . P27,P38

Resende M................... P43

Restelli V..................... P27

Reyes R................... A6

Ricci G...................... P25

Richardson D.................... 3

Richardson SE . . . . . . . . . . . . . . J3

Ritchie G................. C4,J2

Robson HG . . . . ................ SP15

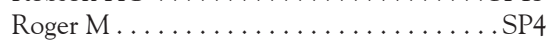

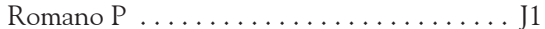

Romney MG ................. C4,SP1

Roscoe D . . . . . . . . . . . . . D1,E3,P7

Roth V . . . . . . . . . . . G4,G5,G6,P2,P42

Rotstein C . . . . . . . . . . . . . . . P43

Rouleau D .....................SP4

Rousseau Y..................... P65

Rubenstein E . . . . . . . . . . . . . . . . . P66

Rutherford C . . . . . . . . . . . . . . . P50

Ryding D ................... P63

$\mathrm{S}$

Salmon J. ................ CVP1

Sarabia A . . . . . ........... C1,P46

Savchenko A . . . . . . . . . . . . . . . P46

Saxinger $\mathrm{L} \ldots \ldots \ldots \ldots \ldots$ A1,P1,P2,P3,P39

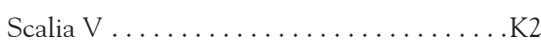

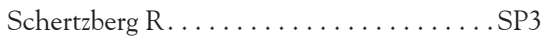

Schoer K . . . . . . . . . . . . . . . . . . . . 33

Sealy D.................... J2

Severini A . .............B3,P54,P60

Sharma M ............. P18,SP14

Sharma N. . . . . . . . . . . . . . . . . . J1

Shaw L........................ . P49

Sheppard DC .................. P65

Shing F. . . . . . . . . . . . . . P7

Shokoples S . . . . . . ............. 1

Show R......................... B2

Simard É.................... P17

Simor A ..........A4,D1,D3,E2,E3,

$\ldots \ldots \ldots \ldots \ldots$ E6,F2,G2,P4,P25,P34,SP29

Singh C . . . . ................ H2

Sirard S . . . . . . . . . . . . . . SP16

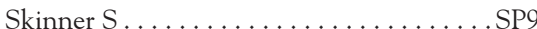

Small G....................... P22

Smart G...................... P2O

Smart N . . . . . . . . . . . . . . . . . . . SP14

Smieja M . . . . . . . . . . . . . G2,P48

Smith $L \ldots \ldots \ldots \ldots \ldots \ldots \ldots \ldots \ldots \ldots$ B2

Smith $\mathrm{S} \ldots \ldots \ldots \ldots \ldots \ldots$ J1,J2,P9,SP6

Solomon N. . . . . . . . . . . . . . . . . . P5

Srigley J . . . . . . . . . . . B7,P6,SP20

St-Antoine P . . . . . . . . . . . . . . SP4

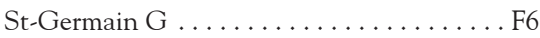

St-Jean M....................SP23

Stojios P......................... 46

St-Pierre G. . . . . . . . . . . . . . . P17

Straus K . . . . . . . . . . . . . . . SP3

Streitenberger L . . . . . . . . . . . . . . . SP5

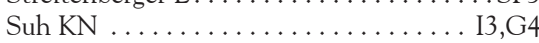

Sullivan L. . . . . . . . . . . . . . . . . . P49

Sumner J .................... B1

\section{$\mathrm{T}$}

Tambalo D . . . . . . . . . . . . . . . . SP24

Tang P . . . . . . . . D 4, G1,G3,K4,P55,P62

Taruc J . . . . . . . . . . . . . . . . . . C4

Taylor G. . . . . . . D1,E3,G5,G6,I3,P2,P39,P42

Taylor M. . . . . . . . . . . . . . . . . P32

Tellier R..................... B4,P59

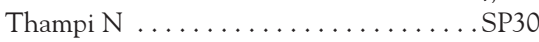

Thanabalan R .................. P22

Thibault L . . . . . . . . . . . . . . . . . . P58

Thirion DJG ............. SP15,P65

Thiry $\mathrm{D} \ldots \ldots \ldots \ldots \ldots \ldots \ldots \ldots \ldots$. . . . . . . . 8

Thliza S ................... I4

Thomas E................... D1,E3

Tipples G . . . . . . . . . . . . P55,P56

Tomalty L. . . . . . . . . . . . . . . . . . . P26

Toor R ........................... B1

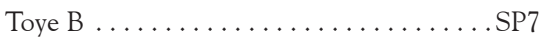

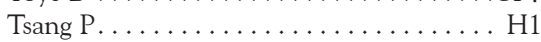

Tuite A.....................

Turcotte P. . . . . . . . . . . . . . . . . B6

Turenne $\mathrm{C} \ldots \ldots \ldots \ldots \ldots \ldots \ldots \ldots$. . . . . . . . .

Turgeon N ................... D1,E3

Turnbull L $\ldots \ldots \ldots \ldots \ldots \ldots \ldots \ldots \ldots \ldots$ P23,P27

Turnbull LA . . . . . . . . . . . . . . . . . P38

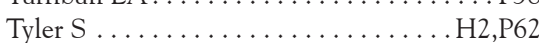

Tyrrell G.....................F 4

\section{$\mathrm{U}$}

Ulanova M

\section{$\mathrm{V}$}

Valiquette L . . . . . . . . . . . . . . . . . . . . . . . SP16

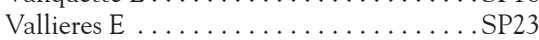

Van Caeseele P . . . . . . . . . . . H5,H6,P20

Van Domselaar G. .............. H2

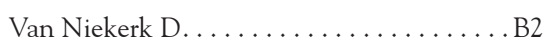

Vandijk A...................... P63

Vaudry W...................... I2

Vearncombe M. .........G2,G5,G6,P42

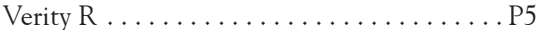

Vermeiren C............... P19,P25

Vernich L........................

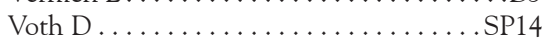

\section{W}

Walker I........................ J4

Walker M.................... H2

Walker SAN ................ E6,P4

Walker SE ...................... E6,P4

Walkty A .................... E1

Wang B. . . . . . . . . . . . . . . . .

Warren $\mathrm{K} \ldots \ldots \ldots \ldots \ldots \ldots \ldots \ldots \ldots$. . . . . . . . .

Warren T ........................SP19

Waters V . . . . . . . . . . . . . . . SP19

Watt C...................... P25,P34

Weber R..................... P54

Webster D . . . . . . . . . . . . . . . CVP1

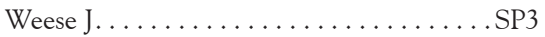

Weir C ...................... G6

Weiss K.................. P2,SP8

Welling S . . . . . . . . . . . . . . . . P41

Westerberg B .................. P27

Wiebe D.............E4,H3,P8,SP31

Wierzbowski AK ...................

Wilcox L ................... P6

Wilkinson K...............G5,I3,P42

Willey BM . . . C C3,P22,P24,P25,P29,P36,SP11

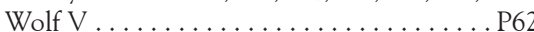

Wolfe J . . . . . . . . . . . . . . P18,SP14

Wong A $\ldots \ldots \ldots \ldots \ldots \ldots$ C4,E2,I3,P2

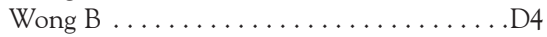

Wong CE . . . . . . . . . . . . . . . . . P33

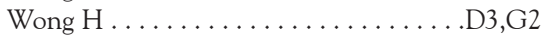

Wong J . . . . . . . . . . . . . . . . . . . . . . .D4

Wong K $\ldots \ldots \ldots \ldots \ldots \ldots \ldots$ P22,P25,P36

Wong M.................. J2

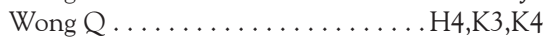

Wong $\mathrm{S} \ldots \ldots \ldots \ldots \ldots \ldots \ldots \ldots$ B4,P59

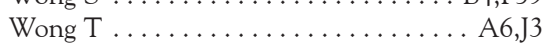

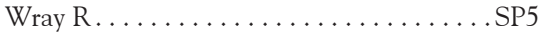

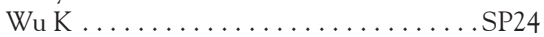

$\mathrm{Y}$

Yamamura D..............J4,SP20

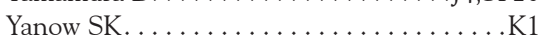

Yau Y .............. SP5,SP19,SP30

Yeboah A ........................ 3

Yow $O \ldots \ldots \ldots \ldots \ldots \ldots \ldots \ldots \ldots \ldots \ldots \ldots$

\section{$\mathrm{Z}$}

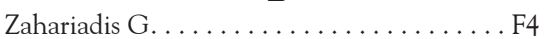

Zhanel GG.................. E1,L2

Zhang K ....................... SP24

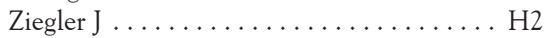

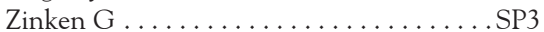

Zoutman D................. E2,P26 


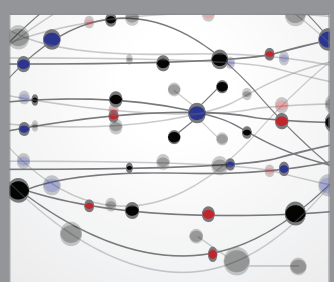

The Scientific World Journal
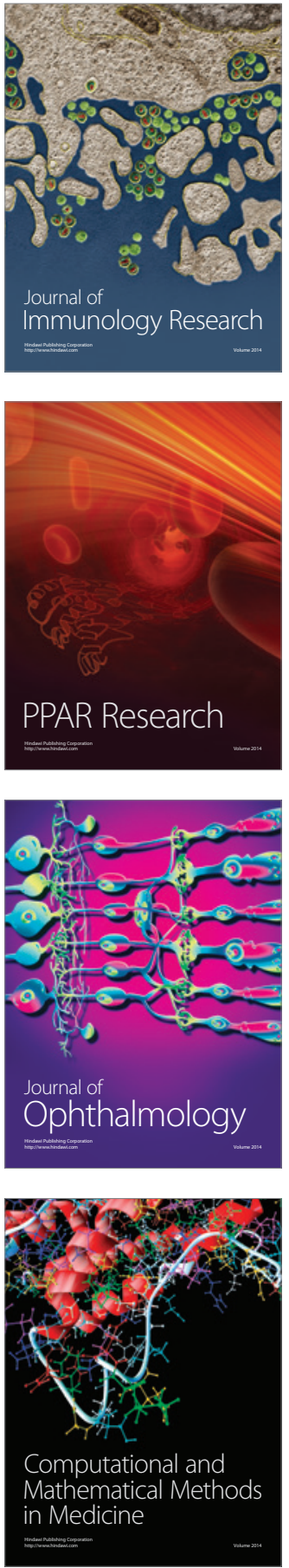

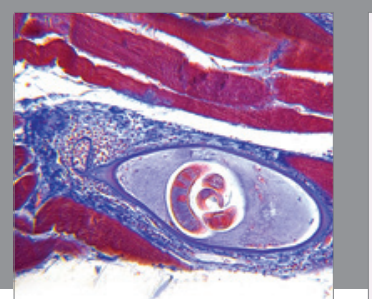

Gastroenterology Research and Practice

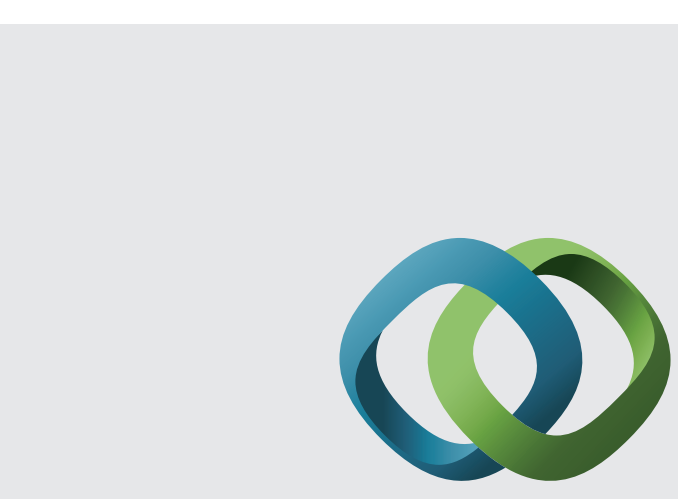

\section{Hindawi}

Submit your manuscripts at

http://www.hindawi.com
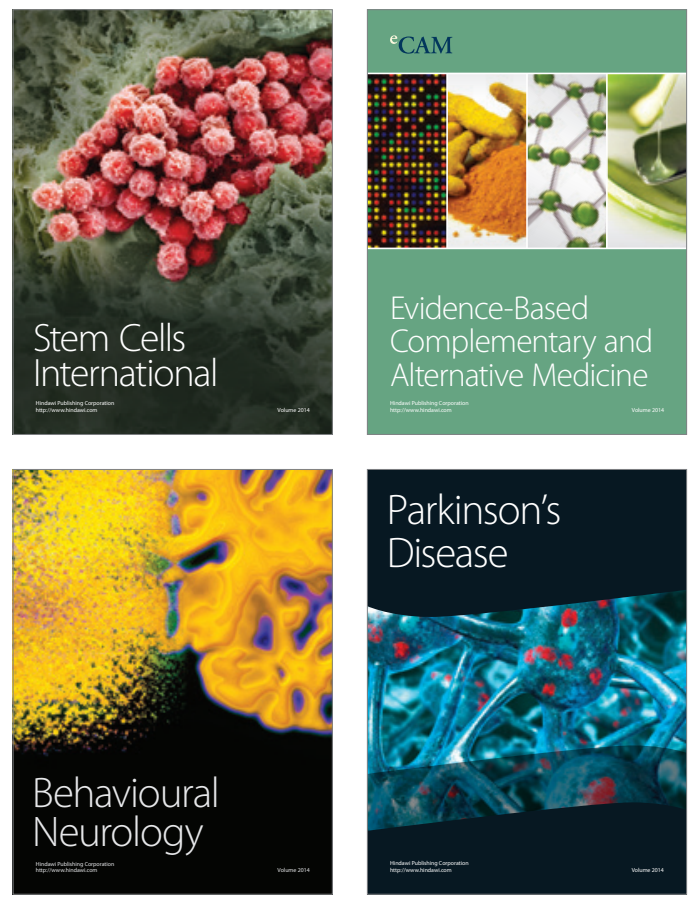
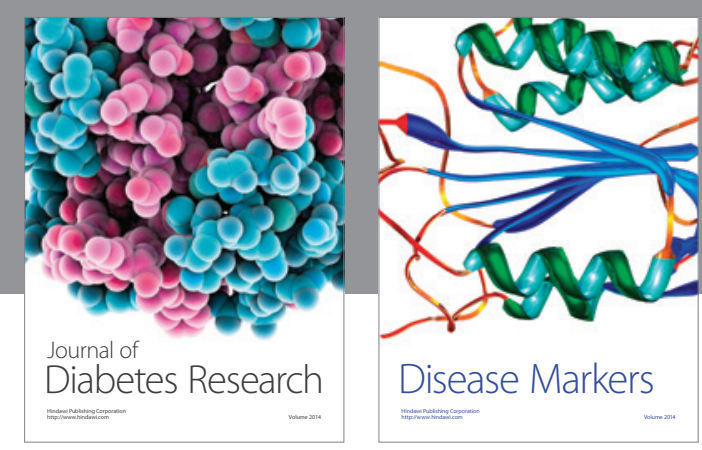

Disease Markers
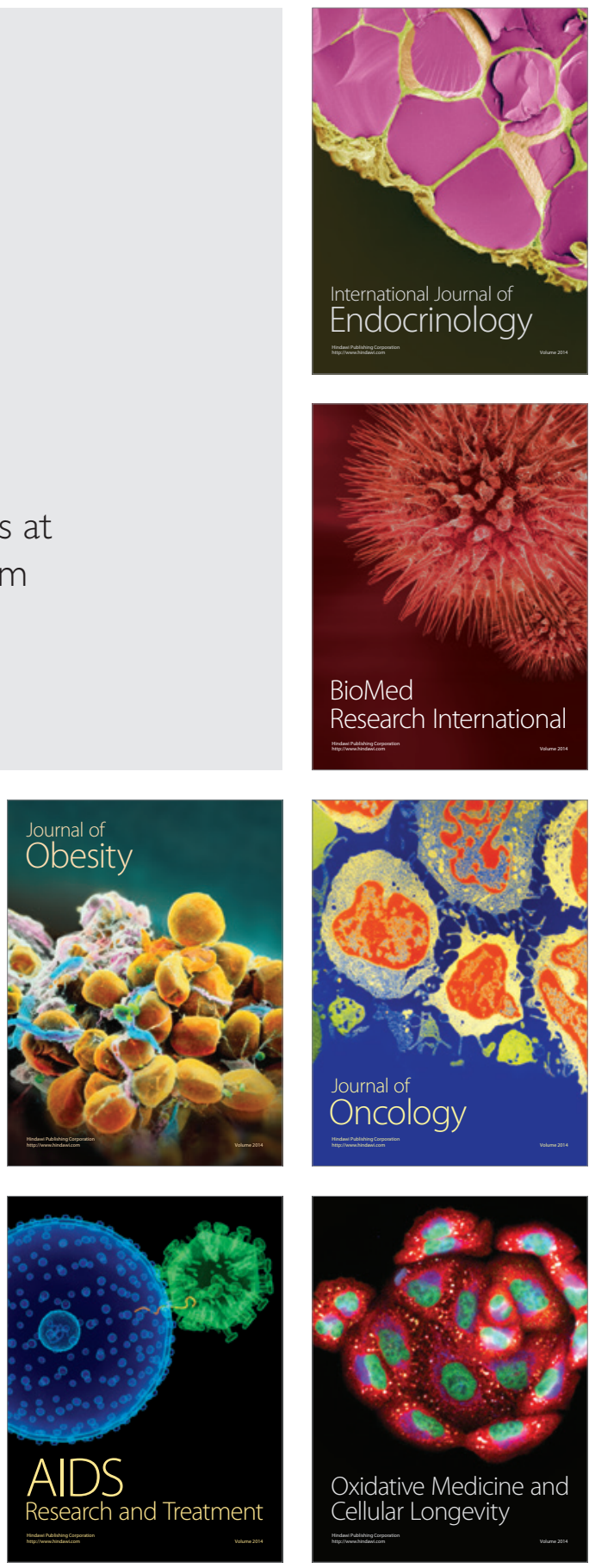